\title{
Métodos Sem Malha e Método dos Elementos Finitos Generalizados em Análise Não-Linear de Estruturas
}

Felício Bruzzi Barros

Tese apresentada à Escola de Engenharia de São Carlos da Universidade de São Paulo, como parte dos requisitos para a obtenção do título de Doutor em Engenharia de Estruturas.

ORIENTADOR: Prof. Dr. Sergio Persival Baroncini Proença

São Carlos 
À minha família: Dora, pais e irmão. 


\section{Agradecimentos}

Ao Prof. Sergio Persival Baroncini Proença pela orientação precisa e competente, pelo tempo dedicado e amizade concedida. Por compreender as particularidades de minhas viagens e se preocupar com algo mais do que apenas o doutorado.

Ao Prof. Clovis Sperb de Barcellos pela paciência e disponibilidade guiando-me pelo "matematiquês" da formulação do Método dos Elementos Finitos.

Aos funcionários do Departamento de Estruturas da Escola de Engenharia de São Carlos e, especialmente, à Maria Nadir Minatel pela relação cordial e simpatia nos auxílios prestados.

Àqueles que conheci e que foram mais do que simplesmente colegas de curso. Agradeço, de modo particular, às minhas colegas de sala, Cristina, Maria Cristina e Ana Rita pela amizade sincera e companheirismo, concedendo um significado especial aos anos dedicados ao doutorado.

Ao CNPq pelo apoio financeiro.

Aos meus Pais pelo apoio integral, fonte inesgotável de incentivos e conselhos, viabilizadores das idas e vindas Minas-São Paulo. Ao meu irmão, Paulo José, pela amizade enriquecida à distância e a mão estendida em qualquer ocasião.

À Dora pelo tempo de namoro e casamento ainda que intermitente, pelos telefonemas, visitas e sonhos compartilhados. Pela espera conduzida com muito amor e delicadeza.

A Deus por enriquecer minha vida com tantas pessoas e possibilidades. Por tudo que tenho a agradecer, entre vitórias e fracassos. 


\section{Resumo}

BARROS, F. B. (2002). Métodos Sem Malha e Método dos Elementos Finitos Generalizados em Análise Não-Linear de Estruturas. São Carlos. 222p. Tese (Doutorado) - Escola de Engenharia de São Carlos, Universidade de São Paulo.

O Método dos Elementos Finitos Generalizados, MEFG, compartilha importantes características dos métodos sem malha. As funções de aproximação do MEFG, atreladas aos pontos nodais, são enriquecidas de modo análogo ao refinamento $p$ realizado no Método das Nuvens $h p$. Por outro lado, por empregar uma malha de elementos para construir as funções partição da unidade, ele também pode ser entendido como uma forma não convencional do Método dos Elementos Finitos. Neste trabalho, ambas as interpretações são consideradas. Os métodos sem malha, particularmente o Método de Galerkin Livre de Elementos e o Método das Nuvens $h p$, são introduzidos com o propósito de estabelecer os conceitos fundamentais para a descrição do MEFG. Na seqüência, apresentam-se aplicações numéricas em análise linear e evidenciam-se características que tornam o MEFG interessante para a simulação da propagação de descontinuidades. Após discutir os modelos de dano adotados para representar o comportamento não-linear do material, são introduzidos exemplos de aplicação, inicialmente do Método das Nuvens $h p$ e depois do MEFG, na análise de estruturas de concreto. Os resultados obtidos servem de argumento para a implementação de um procedimento p-adaptativo, particularmente com o MEFG. Propõe-se, então a adaptação do Método dos Resíduos em Elementos Equilibrados à formulação do MEFG. Com vistas ao seu emprego em problemas não-lineares, algumas modificações são introduzidas à formulação do estimador. Mostra-se que a medida obtida para representar o erro, apesar de fundamentada em diversas hipóteses nem sempre possíveis de serem satisfeitas, ainda assim viabiliza a análise não-linear $p$-adaptativa. Ao final, são enumeradas propostas para a aplicação do MEFG em problemas caracterizados pela propagação de defeitos.

Palavras-chave: Método dos Elementos Finitos; Métodos sem Malha; Análise nãolinear; Mecânica do Dano; Estimador de Erro; Adaptatividade. 


\section{Abstract}

BARROS, F. B. (2002). Meshless Methods and Generalized Finite Element Method in Structural Nonlinear Analysis. São Carlos. 222p. Thesis (Doctoral) - São Carlos School of Engineering, University of São Paulo.

The Generalized Finite Element Method, GFEM, shares several features with the so called meshless methods. The approximation functions used in the GFEM are associated with nodal points like in meshless methods. In addition, the enrichment of the approximation spaces can be done in the same fashion as in the meshless $h p$-Cloud method. On the other hand, the partition of unity used in the GFEM is provided by Lagrangian finite element shape functions. Therefore, this method can also be understood as a variation of the Finite Element Method. Indeed, both interpretations of the GFEM are valid and give unique insights into the method. The meshless character of the GFEM justified the investigation of meshless methods in this work. Among them, the Element Free Galerkin Method and the $h p$-Cloud Method are described aiming to introduce key concepts of the GFEM formulation. Following that, several linear problems are solved using these three methods. Such linear analysis demonstrates several features of the GFEM and its suitability to simulate propagating discontinuities. Next, damage models employed to model the nonlinear behavior of concrete structures are discussed and numerical analysis using the $h p$-Cloud Method and the GFEM are presented. The results motivate the implementation of a $p$-adaptive procedure tailored to the GFEM. The technique adopted is the Equilibrated Element Residual Method. The estimator is modified to take into account nonlinear peculiarities of the problems considered. The hypotheses assumed in the definition of the error measure are sometimes violated. Nonetheless, it is shown that the proposed error indicator is effective for the class of $p$-adaptive nonlinear analysis investigated. Finally, several suggestions are enumerated considering future applications of the GFEM, specially for the simulation of damage and crack propagation.

Keywords: Finite Element Method; Meshless Method; Nonlinear Analysis; Damage Mechanics; Error Estimator; Adaptivity. 


\section{Sumário}

Lista de Figuras

Lista de Tabelas vii

Lista de Símbolos viii

1 Introdução 1

1.1 Considerações Iniciais $\ldots \ldots \ldots 1$

1.1.1 Quanto aos Métodos Numéricos: Sem Malha e dos Elementos Finitos Generalizados . . . . . . . . . . . . . . . 2

1.1.2 Quanto à Mecânica dos Materiais . . . . . . . . . . 5

1.2 Organização do Texto . . . . . . . . . . . . . . . . . 6

2 Fundamentos dos Métodos Numéricos 8

2.1 Método dos Mínimos Quadrados Móveis (MMQM) . . . . . . . . . . 9

2.2 Famílias de Funções do Método das Nuvens $h p\left(\Im_{N}^{k, p}\right) \ldots \ldots$

2.3 Formulação de Galerkin para Problemas de Valor de Contorno e Emprego dos Métodos Sem Malha . . . . . . . . . . . . . . . 14

2.4 Integração Numérica . . . . . . . . . . . . . . . . . 16

2.5 Método dos Elementos Finitos Generalizados . . . . . . . . . . . 18

2.5.1 A Formulação . . . . . . . . . . . . . . . . . . . . 19

3 Experimentos Numéricos em Análise Linear 23

3.1 Métodos Sem Malha Aplicados no Estudo da Associação Contínua de Painéis Parede e Pórtico . . . . . . . . . . . . . . . . 24

3.1 .1 Análise numérica . . . . . . . . . . . . 27

3.2 Problemas de Elasticidade Linear Estática - Análise Bi-Dimensional pelo MEFG . . . . . . . . . . . . . . . . . . . . 32 
3.2.1 Efeito da Distorção da Malha de Elementos . . . . . . . . . 36

3.2.2 Chapa com Orifício . . . . . . . . . . . . . . 41

3.2.3 Cisalhamento de uma Chapa . . . . . . . . . . . . . 44

3.2 .4 Chapa em L . . . . . . . . . . . . . . 47

3.2.5 Considerações Complementares . . . . . . . . . . . 52

4 Modelo Constitutivo $\quad 54$

4.1 Conceitos da Mecânica do Dano Contínuo . . . . . . . . . . . . . . . 55

4.2 Modelo de Mazars . . . . . . . . . . . . . . . . . . . 56

4.3 Modelo de La Borderie . . . . . . . . . . . . . . . . . 59

4.4 Abordagem Não-Local . . . . . . . . . . . . . . . . . . 61

5 Experimentos Numéricos em Análise Não-Linear 65

5.1 Viga em Concreto Armado . . . . . . . . . . . . . . . . 66

5.2 Análise pelo Método das Nuvens $h p \ldots \ldots 7$

5.2.1 Problema Não-Linear . . . . . . . . . . . . . . 70

5.2.2 Integração Numérica na Seção Transversal . . . . . . . . . 71

5.2.3 Análise Numérica . . . . . . . . . . . . . . . 73

5.3 Análise Bi-dimensional com do MEFG . . . . . . . . . . 76

5.3.1 Análise Numérica . . . . . . . . . . . . . . 77

5.4 Chapa de Concreto Tracionada . . . . . . . . . . . . . . . 83

$6 \quad$ Estimador de Erro $\quad \mathbf{8 8}$

6.1 Conceitos e Definições para o Estudo de Erro . . . . . . . . . . . . . 89

6.2 Estimadores de Erro e Adaptatividade . . . . . . . . . . . . . . . . 92

6.2.1 Estimadores de Erro no MEF . . . . . . . . . . . . . . . . . 94

6.2.2 Estimadores de Erro no MEFG . . . . . . . . . . . . . . . 97

6.2.3 Estimador de erro no MEFG - Escolha e Justificativa . . . . . 98

6.3 Método dos Resíduos em Elementos . . . . . . . . . . . . . . . . . 99

6.4 Equilíbrio dos Resíduos . . . . . . . . . . . . . . . . . . . . 106

6.4.1 Estratégia de Equilíbrio de Ladevèze \& Maunder . . . . . . . 107

6.4 .2 Algoritmo Adaptativo . . . . . . . . . . . . . 113

6.5 Exemplos Numéricos . . . . . . . . . . . . . . . . . . . . 117

6.5 .1 Viga Engastada . . . . . . . . . . . . . . 117

6.5.2 Chapa com Orifício . . . . . . . . . . . . . . 122 
6.6 Medida de Erro em Análise Não-Linear . . . . . . . . . . . . . . . 126

6.6.1 Estratégia de Estimativa do Erro . . . . . . . . . . . . 127

6.6 .2 Algoritmo Adaptativo . . . . . . . . . . . . . 133

6.6.3 Considerações sobre a Transferência das Variáveis . . . . . . 136

6.7 Exemplo Numérico . . . . . . . . . . . . . . . . . . . . . . . . . . . 139

6.7.1 Chapa de Concreto Comprimida . . . . . . . . . . . . . . 141

6.7.2 Chapa com Entalhe . . . . . . . . . . . . . . 145

7 Considerações Finais

7.1 Síntese e Conclusões . . . . . . . . . . . . . . . . . . . 152

7.2 Dano e Fratura . . . . . . . . . . . . . . . . . . . . 154

7.3 Outras Propostas de Futuros Desenvolvimentos . . . . . . . . . . 158

$\begin{array}{ll}\text { Referências Bibliográficas } & 161\end{array}$

A Formulação Tangente para o Modelo de Mazars 1

B Partição da Unidade $\quad 4$

C Solução do Sistema de Equações no MEFG 6

$\begin{array}{lr}\text { D Espaço de Aproximação Polinomial } & 10\end{array}$

$\begin{array}{llr}\text { E Discussão Sobre o Erro A Priori } & 11\end{array}$

F Detalhes da Implementação do MEFG

F.1 Convergência no Método de Newton-Raphson . . . . . . . . . . . . . 13

F.2 Consideração da armadura . . . . . . . . . . . . . . . . 15

G Interpretação do Estimador do MRE

H Equilíbrio do Vetor de Forças Generalizadas $\quad 18$

I Transferência de Valores Associados aos Pontos de Gauss 21

J Solução na Vizinhança de Trinca em Elasticidade Bi-Dimensional 23 


\section{Lista de Figuras}

2.1 Método dos Mínimos Quadrados Móveis . . . . . . . . . . . . . 9

2.2 Representação das nuvens em $\mathbb{R}^{2} \ldots \ldots$. . . . . . . . . . . 10

2.3 Setores da função de forma $\phi_{j}$ - parâmetros empregados: $m=3$, $R_{j}=1,6 h, h \rightarrow$ distância entre os nós - Observar que $\phi_{j}$ verifica a condição do $\delta_{j i}$ para o caso ilustrado em que $m=n$, o que nem sempre é verdadeiro. . . . . . . . . . . . . . . . 17

2.4 Partição da Unidade a partir dos elementos finitos em $\mathbb{R}^{1}$. . . . . . . 19

2.5 Esquema de enriquecimento da Partição da Unidade . . . . . . . . . . 20

3.1 Associação parede-pórtico . . . . . . . . . . . . . . . . . . . 24

3.2 Discretização da associação parede e pórtico . . . . . . . . . . . 27

3.3 Influência da rigidez relativa $K_{r} \ldots \ldots \ldots \ldots$

3.4 Geometria das estruturas propostas - sem unidades . . . . . . . 36

3.5 Discretizações propostas . . . . . . . . . . . . . . 38

3.6 Chapa com orifício . . . . . . . . . . . . . . . . 43

3.7 Gráficos comparativos MEF x MEFG . . . . . . . . . . . . 45

3.8 Estudo quanto ao travamento de Poisson . . . . . . . . . . . . . . 46

3.9 Análise quanto ao travamento de Poisson . . . . . . . . . . . . 46

3.10 Chapa em L - geometria e malhas utilizadas nas seqüências de refinamento consideradas . . . . . . . . . . . . . . . 48

3.11 Análises para diversos tipos de refinamento . . . . . . . . . 51

4.1 Elemento de volume representativo . . . . . . . . . . . . 55

4.2 Relação constitutiva . . . . . . . . . . . . . . . . . . . . . . 57

4.3 Representação das diferenças entre análise local e não-local. Curvas de força $F$ e deslocamento no meio do vão $u$, com a respectiva distribuição de dano . . . . . . . . . . . . . . . . . . 
4.4 Análise não-local em estrutura com simetria . . . . . . . . . . . . . . 64

5.1 Viga em concreto armado - geometria e armação - medidas em cm . . 66

5.2 Abordagem uni-axial . . . . . . . . . . . . . . . . . 67

5.3 Sistema de estratos . . . . . . . . . . . . . . . . 72

5.4 Análises estáticas - resultados experimentais de ÁLVARES (1993) . . 74

5.5 Análises dinâmicas . . . . . . . . . . . . . . . . . . 75

5.6 Condições de contorno e malha de elementos adotadas . . . . . . . . 77

5.7 Curvas de $\sigma \times \varepsilon$ variando-se $A_{T} \ldots \ldots \ldots$. . . . . . . 80

5.8 Análise estática . . . . . . . . . . . . . . . . 81

5.9 Mapa da distribuição do dano . . . . . . . . . . . . . . . . . 82

5.10 Chapa de concreto - geometria e condições de contorno - medidas em

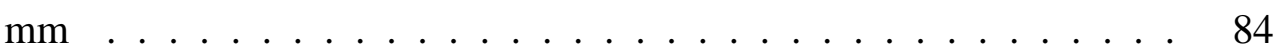

5.11 Malhas utilizadas para a análise - medidas em mm . . . . . . . . 85

5.12 Mapa da distribuição do dano . . . . . . . . . . . . . . . 86

5.13 Superfície representativa do dano . . . . . . . . . . . . . . . . 87

6.1 Decomposição de $\hat{\boldsymbol{\Theta}}_{j}^{\mathcal{K}}={ }^{a} \hat{\boldsymbol{\Theta}}_{j}^{\mathcal{K}}+{ }^{b} \hat{\boldsymbol{\Theta}}_{j}^{\mathcal{K}} \ldots \ldots \ldots \ldots . \ldots \ldots$

6.2 Processo de balanceamento das forças $\hat{\Theta}_{j}^{\mathcal{K}}$ respeitando-se o equilíbrio nodal ............................ 111

6.3 Malha formada por 10 elementos regulares quadrangulares . . . . . 117

6.4 Índices locais de efetividade da malha da Figura 6.3, para cada elemento 119

6.5 Índice de efetividade de $\mathrm{MRE}_{2}$ para uma seqüencia de 3 malhas aninhadas ........................... 121

6.6 Índices locais de efetividade - Malha de elementos distorcidos . . . . 122

6.7 Malha de elementos adotada . . . . . . . . . . . . . . . 123

6.8 Índices locais de efetividade - malha com aproximação linear . . . . . 123

6.9 Erro relativo para as iterações dos refinamentos adaptativos . . . . . . 125

6.10 Resultado final do refinamento $p$-adaptativo, $\mathrm{MRE}_{p+1} \ldots \ldots$. . . . 125

6.11 Interpretação geométrica para a estimativa do erro no caso uni-axial 129

6.12 Chapa de concreto - geometria e condições de contorno - medidas em $\mathrm{mm} \ldots \ldots \ldots \ldots \ldots \ldots \ldots 14 \ldots \ldots \ldots \ldots$

6.13 Relação uni-axial tensão $\times$ deformação . . . . . . . . . . . . . . . . 142

6.14 Malhas utilizadas - medidas em mm . . . . . . . . . . . . . . . 143

6.15 Resposta global da estrutura . . . . . . . . . . . . . . . 144 
6.16 Mapa da distribuição do dano no passo 41 . . . . . . . . . . . . 145

6.17 Índice de efetividade global em cada passo . . . . . . . . . . . . . 145

6.18 Chapa de concreto com entalhe - geometria e solicitação - medidas em $\mathrm{mm} \ldots \ldots \ldots \ldots \ldots \ldots \ldots \ldots$

6.19 Malha e condições de contorno utilizadas para a análise . . . . . . . 147

6.20 Resposta global da estrutura . . . . . . . . . . . . . . . . . . . . . . 149

6.21 Discretizações definidas pelo algoritmo adaptativo . . . . . . . . . . . 150

6.22 Distribuição do dano ao final do carregamento $(F=18,5 \mathrm{kN}) \quad \ldots$

7.1 Malha e condições de contorno utilizadas para a análise . . . . . . 155

7.2 Confrontação dano $\times$ fratura . . . . . . . . . . . . . 156

7.3 Zona de processo de danificação . . . . . . . . . . . . . . 157

D.1 Monômios representados _ . . . . . . . . . . . . . . . . 10

F.1 Aço embutido nos elementos finitos com o material concreto . . . . 15 


\section{Lista de Tabelas}

3.1 Tempo de processamento para as várias distribuições nodais (NN) com os métodos sem malha $-K_{r}=20, p=2 \ldots \ldots \ldots$

3.2 Esforço cortante no pórtico $-K_{r}=20$, base $\boldsymbol{P}_{k=3} \ldots \ldots \ldots$

3.3 Esforço momento fletor na parede- $K_{r}=20$, base $\boldsymbol{P}_{k=2} \ldots \ldots \ldots$

3.4 Esforço cortante no pórtico - $K_{r}=20$, família $\Im_{N}^{k=0, p=2} \ldots \ldots 31$

3.5 Esforço momento fletor na parede $-K_{r}=20$, família $\Im_{N}^{k=0, p=2} \ldots 32$

3.6 Resultados para o MEF de LEE; BATHE (1993) e MEFG . . . . . . . 39

3.7 Resultados comparativos para a energia de deformação normalizada, entre o MEF, SZABÓ; BABUŠKA (1991) e o MEFG, $\left(\mathcal{U}_{p} E / \sigma_{\infty}^{2} a^{2} t\right)$. Solução analítica exata normalizada de SZABÓ; BABUŠKA (1991) $\mathcal{U E} / \sigma_{\infty}^{2} a^{2} t=7,69365 \ldots \ldots \ldots \ldots \ldots \ldots$

3.8 Análises pelo MEFG enriquecido polinomialmente. Para $\nu=0,3000$ tem-se $\mathcal{U}=0,130680 \mathrm{~N} \times \mathrm{mm}$ e para $\nu=0,4999, \mathcal{U}=0,127035 \mathrm{~N} \times$ mm, DUARTE (1991). . . . . . . . . . . . . . .

3.9 Resultados numéricos comparativos para a energia de deformação normalizada, $\left(\mathcal{U}_{p} E / A_{1}^{2} a^{2 \lambda_{1}} t\right)$, entre o MEF, SZABÓ; BABUŠKA (1991) e o MEFG. Solução analítica exata de $U E / A_{1}^{2} a^{2 \lambda_{1}} t=4,15454423$ obtida de SZABÓ; BABUŠKA; CHAYAPATHY (1989) . . . . . . . .

3.10 Resultados para a energia de deformação, $\left(\tilde{\mathcal{U}} E / A_{1}^{2} a^{2 \lambda_{1}} t\right)$, através do MEFG. Solução analítica exata, SZABÓ; BABUŠKA; CHAYAPATHY (1989) $U E / A_{1}^{2} a^{2 \lambda_{1}} t=4,15454423 \ldots \ldots \ldots \ldots \ldots$. . . . . . . .

5.1 Parâmetros do material $(c \rightarrow$ concreto e $s \rightarrow$ aço $) \ldots . \ldots 66$

6.1 Índices globais de efetividade para as iterações dos refinamentos adaptativos $-\mathrm{MRE}_{p+1} \ldots \ldots \ldots \ldots \ldots \ldots$ 


\section{Lista de Símbolos}

$A_{\alpha} \quad$ parâmetros do material no modelo de La Borderie a ser identificado

$A_{C}, B_{C}$ parâmetros característicos do material na compressão uni-axial para o modelo de Mazars

$A_{T}, B_{T}$ parâmetros característicos do material na tração uni-axial para o modelo de Mazars

$B_{\alpha} \quad$ parâmetros do material no modelo de La Borderie a ser identificado

$b_{i} y \quad$ parâmetros nodais de deslocamentos generalizados, das funções enriquecedoras

$C, C_{\infty}$ e $C_{G}$ constantes usadas na estimativa a priori do erro

$C^{k} \quad$ Espaço de funções com derivadas contínuas até a ordem $k$

$C_{0}^{l} \quad$ Espaço de funções com derivadas de suporte compacto e contínuas até a ordem $l$

$C_{0}^{\min (k, l)}$ Espaço de funções com derivadas de suporte compacto e contínuas até a ordem $\min (k, l)$

$c_{i} \quad$ parâmetros nodais generalizados das funções teste enriquecedoras

$c_{j \alpha}^{x} \mathrm{e} c_{j \alpha}^{y}$ parâmetros associados às funções de trinca para o modo II

D variável escalar de dano

$D_{1}$ e $D_{2}$ variáveis de dano à tração e compressão do modelo de La Borderie

$d_{a} \quad$ tamanho do maior agregado no concreto

$D_{C} \quad$ dano associado ao estado uni-axial de compressão no modelo de Mazars

$D_{n} \quad$ Variável de dano segundo a direção do vetor $\vec{n}$

$D_{T} \quad$ dano associado ao estado uni-axial de tração no modelo de Mazars

$d_{j \alpha}^{x}$ e $d_{j \alpha}^{y}$ parâmetros associados às funções de trinca para o modo I

$d_{j}^{x}$ e $d_{j}^{y}$ parâmetros associados às funções não polinomiais introduzidas ao enriquecimento

$E_{0} \quad$ módulo de elasticidade do material íntegro

F força concentrada

$f$ função prescrita em $\Omega$ para o PVC 
$F_{\alpha} \quad$ critério de evolução de $D_{\alpha}(\alpha=1,2)$

$F_{f} \quad$ parcela da força $F$ absorvida pelo pórtico

$F_{w} \quad$ parcela da força $F$ absorvida pela parede

$f_{d} \quad$ função que descreve as condições de abertura e fechamento das micro-fissuras no modelo de La Borderie

G módulo de elasticidade transversal

$g(\boldsymbol{x}) \quad$ função de ponderação empregada na análise não-local

$G_{1}\left(z_{1}\right)$ e $G_{2}\left(z_{2}\right)$ funções de encruamento no modelo de La Borderie

$G_{j} \quad$ conjuntos abertos que definem o suporte das funções da PU

$g_{k} \quad$ funções prescritas em $\partial \Omega$ para o PVC

$H \quad$ altura da estrutura formada pela associação parede e pórtico

$h \quad$ dimensão característica da discretização do domínio

$h_{\min } \min _{j=1, \cdots, N} h_{j}$

$h_{j} \quad$ dimensão característica associada à nuvem $\omega_{j}$

$i_{\text {fim }} \quad$ contador que se for igual ao número de nuvens ou elementos indica a divergência do procedimento adaptativo

$J(\boldsymbol{x}) \quad$ funcional do erro ponderado de aproximação em $\boldsymbol{x}$

$j_{w} \quad$ regidez à flexão

$K_{r} \quad$ equivale a $k_{r} H$

$k_{r} \quad$ rigidez relativa entre o pórtico e a parede

$L_{\alpha} \quad$ tamanho do lado $\alpha$ do elemento finito

$l_{c} \quad$ comprimento característico do material

$L_{j i} \quad$ corresponde à i-ésima função que multiplica a PU do nó $\boldsymbol{x}_{j}$, podendo ser polinomial ou não

$m \quad$ número de funções que forma a base $\boldsymbol{P}$

$N \quad$ número de pontos nodais do conjunto $Q_{N}$

$n(\boldsymbol{x}) \quad$ número de pontos nodais do sub-conjunto $\widehat{Q}_{n(\boldsymbol{x})}$ definido com relação a $\boldsymbol{x}$

$n_{v} \quad$ variável que se for igual a 0 indica que $v$ deve ser diminuído

$p_{\mathcal{K}}$ ordem polinomial mais baixa das funções de aproximação das nuvens que contêm o elemento $\mathcal{K}$

$p_{i} \quad$ função da base $\boldsymbol{P}$ 
$p_{\omega_{j}} \quad$ ordem polinomial da aproximação da nuvem $\omega_{j}$

$q \quad$ força distribuída

$q_{f} \quad$ parcela da força $q$ absorvida pelo pórtico

$q_{j}(p)$ número de funções enriquecedoras no Método das Nuvens $h p$ e MEFG para a nuvem $\omega_{j}$

$q_{j}(p)$ número de polinômios enriquecedores no Método das Nuvens $h p$ e MEFG para a nuvem $\omega_{j}$ e com a ordem polinomial $p$ máxima que se deseja representar

$q_{w} \quad$ parcela da força $q$ absorvida pela parede

$R_{j} \quad$ raio da região de influência ou nuvem do ponto $\boldsymbol{x}_{j}$

$r_{n l} \quad$ raio da análise não-local

$S_{\beta} \quad$ conjunto de funções obtidas pelo produto de $\mathcal{J}_{j}$ pela PU no elemento $\beta$

$s_{f} \quad$ rigidez ao cisalhamento

$t \quad$ tempo

$T O L_{\text {erro }}$ tolerância admitida para o erro relativo global

$T O L_{\mathcal{u}}$ tolerância para a energia de deformação usada para o controle do equilíbrio no Método de Newton-Raphson

$u(\boldsymbol{x}) \quad$ Função contínua

$u_{i}^{x} \quad$ parâmetros nodais de deslocamentos generalizados

$u_{j} \quad$ valores que $u(\boldsymbol{x})$ assume em $\boldsymbol{x}=\boldsymbol{x}_{j}$

$u_{p}(\boldsymbol{x})$ Função aproximadora que representa exatamente o espaço de polinômios com grau máximo $p$

$u_{x}$ e $u_{y}$ funções de deslocamentos nas direções $x$ e $y$

$u_{x}^{\prime} \quad$ derivada primeira dos deslocamentos em $x, \operatorname{com}$ relação à direção $x$

$u_{x}^{\prime \prime} \quad$ derivada segunda dos deslocamentos em $x$, com relação à direção $x$

$u_{y}^{\prime} \quad$ derivada primeira dos deslocamentos em $y$, com relação à direção $x$

$u_{y}^{\prime \prime} \quad$ derivada segunda dos deslocamentos em $y$, com relação à direção $x$

$v \quad$ funções de teste do Método de Galerkin

$V\left(r_{n l}\right)$ volume do material compreendido dentro de uma região de raio $r_{n l}$

$v_{i} \quad$ parâmetros nodais generalizados das funções teste

$v_{x} \mathrm{e} v_{y}$ funções de teste nas direções $x$ e $y$

$w\left(\boldsymbol{x}_{j}\right)$ peso do ponto $\boldsymbol{x}_{j}$ da quadratura de Gauss 
$W_{j} \quad$ função peso do método dos MQM

$Y_{i} \quad$ variável associada a $D_{i}(i=1,2)$ no modelo de La Borderie

$Y_{o \alpha} \quad$ parâmetros do material no modelo de La Borderie a ser identificado

$z_{1}$ e $z_{2}$ medidas de dano acumulado no modelo de La Borderie

$Z_{\alpha} \quad$ variável associada a $z_{\alpha}(\alpha=1,2)$

$\boldsymbol{A}(\boldsymbol{x})$ matriz empregada na formulação do método dos MQM

$\boldsymbol{A}_{\mathcal{N}}$ matriz obtida das funções PU e que estabelece a relação entre $\boldsymbol{b}_{\theta}$ e $\boldsymbol{c}_{\Theta}$

$b$ força de volume

$\boldsymbol{b}_{\theta} \quad$ vetor que contém as tensões nodais ${ }^{a} \hat{\boldsymbol{\theta}}_{j}^{\mathcal{K}}$

$\boldsymbol{B}_{j}(\boldsymbol{x})$ vetor empregado na formulação do método dos MQM

C matriz das propriedades constitutivas

$C_{0} \quad$ matriz com as propriedades elásticas do material íntegro

$c_{\Theta} \quad$ vetor que contém as forças nodais ${ }^{a} \hat{\boldsymbol{\Theta}}_{j}^{\mathcal{K}}$

$\boldsymbol{E} \quad$ tensor de deformações

$e_{i} \quad$ erro aproximado da iteração $i$ do procedimento de Babuška

$\boldsymbol{e}_{p} \quad$ função de erro da aproximação $\boldsymbol{u}_{p}$

$\boldsymbol{G} \quad$ sub-matriz de $\boldsymbol{M}$ nos métodos sem malha, oriunda da aplicação do Método dos Multiplicadores de Lagrange

$\boldsymbol{H}$ vetor que representa a derivada da variável dano com relação às deformações principais

I matriz identidade

$I^{\mathcal{K}} \quad$ vetor dos indicadores de erro nodais generalizados

$\boldsymbol{I}_{\omega_{j}} \quad$ parâmetros da função indicadora de erro $\tilde{\boldsymbol{e}}_{p}$ associados à nuvem $\omega_{j}$

$\boldsymbol{K}^{\mathcal{K}} \quad$ matriz de rigidez do elemento para ordem $p$

$\boldsymbol{K}_{\mathrm{er}}^{\mathcal{K}}$ matriz de rigidez para o problema de cálculo do indicador de erro no MRE

$\boldsymbol{K}_{\epsilon} \quad$ matriz gerada da perturbação de $\boldsymbol{K}$ no procedimento de Babuška

$\boldsymbol{M}_{j}^{\mathcal{K}}$ momento no elemento $\mathcal{K}$ das forças nodais generalizadas $\boldsymbol{F}_{j}$ para cada nível de enriquecimento

$n$ versor que descreve a orientação do contorno

$\boldsymbol{P}$ base de funções

$\boldsymbol{p} \quad$ conjunto de funções $p_{i}$ 
$\boldsymbol{P}_{k} \quad$ conjunto de monômios que geram o espaço $\mathcal{P}_{k}$

$\boldsymbol{P}_{p} \quad$ conjunto de monômios que geram o espaço $\mathcal{P}_{p}$

$\boldsymbol{R}^{\mathcal{K}} \quad$ vetor das forças residuais nodais generalizadas

$\boldsymbol{r}_{\Gamma} \quad$ função dos resíduos do PVC no contorno $\Gamma_{N}$

$\boldsymbol{r}_{\Omega} \quad$ função dos resíduos do PVC no domínio $\Omega$

$\boldsymbol{r}_{i} \quad$ resíduo da iteração $i$ do procedimento de Babuška

$\boldsymbol{t} \quad \boldsymbol{\Sigma} \boldsymbol{n}$, tensão sobre o contorno normal a $\boldsymbol{n}$

$\boldsymbol{U} \quad$ vetor dos parâmetros (deslocamentos) generalizados do problema aproximado

$\boldsymbol{u} \quad\left[\begin{array}{ll}u_{x} & u_{y}\end{array}\right]^{T}$, o vetor das funções de deslocamentos nas direções $x$ e $y$

$\boldsymbol{U}_{i} \quad$ solução da iteração $i$ do procedimento de Babuška

$\boldsymbol{U}_{x}$ e $\boldsymbol{U}_{y}$ vetor dos parâmetros nodais dos deslocamentos generalizados na direção $x$ e $y$

$\boldsymbol{V} \quad$ vetor das parâmetros generalizados das funções de teste

$\boldsymbol{v} \quad\left[\begin{array}{ll}v_{x} & v_{y}\end{array}\right]^{T}$, o vetor das funções de teste nas direções $x$ e $y$

$\boldsymbol{x} \quad$ posição do domínio $\bar{\Omega}$

$\boldsymbol{x}_{p} \quad$ posição do centróide das massas $\left(m_{a}, m_{b}, m_{c}, m_{d}\right)$

$\widehat{\boldsymbol{F}} \quad$ vetor de forças generalizadas para os método sem malha

$\widehat{\boldsymbol{K}} \quad$ matriz de rigidez generalizada para os método sem malha

$\widehat{M} \quad$ matriz de massa consistente generalizada para os métodos sem malha

$\widehat{U} \quad$ vetor que contém os parâmetros nodais generalizados $\boldsymbol{U}$ e os multiplicadores de Lagrange $\lambda$ nos métodos sem malha

${ }^{t} \boldsymbol{I}^{\mathcal{K}} \quad$ vetor dos indicadores de erro nodais generalizados no passo $t$

${ }^{t} \boldsymbol{K}_{\mathrm{er}}^{\mathcal{K}}$ matriz de rigidez para o problema de cálculo do indicador de erro no MRE no passo $t$

${ }^{t} \boldsymbol{R}^{\mathcal{K}}$ vetor das forças residuais nodais generalizadas no passo $t$

${ }^{t} \boldsymbol{r}_{\Gamma}$ função dos resíduos do PVC no contorno $\Gamma_{N}$ para o passo $t$

${ }^{t} \boldsymbol{r}_{\Omega}$ função dos resíduos do PVC no domínio $\Omega$ para o passo $t$

$\boldsymbol{F}_{\text {ext }} \quad$ vetor de forças externas generalizadas no MEFG

$\boldsymbol{F}_{\text {int }} \quad$ vetor de forças internas generalizadas no MEFG

${ }^{t} \tilde{u}^{\left(i_{t}\right)}(\boldsymbol{x})$ valor da função $u$ na iteração $i_{t}$ do passo de tempo $t$

$\boldsymbol{K} \quad$ matriz de rigidez 
$\left(m_{a}, m_{b}, m_{c}, m_{d}\right)$ pesos atrelados a cada um dos lados de um elemento quadrilátero para o equilíbrio do resíduo

$\overline{\boldsymbol{F}} \quad$ vetor obtido a partir de $\boldsymbol{F}$, pela normalização da diagonal de $\boldsymbol{K}$, no procedimento de Babuška

$\overline{\boldsymbol{K}}$ matriz obtida pela normalização da diagonal de $\boldsymbol{K}$, no procedimento de Babuška

$\overline{\boldsymbol{U}} \quad$ vetor obtido a partir de $\boldsymbol{U}$, pela normalização da diagonal de $\boldsymbol{K}$, no procedimento de Babuška

$\ddot{U}_{x}$ e $\ddot{\boldsymbol{U}}_{y}$ vetor dos parâmetros nodais das acelerações generalizados na direção $x$ e $y$

$\ddot{u}_{x} \quad$ aceleração na direção $x$

$\ddot{u}_{y} \quad$ aceleração na direção $y$

$\dot{D} \quad$ taxa de dano

$\dot{u}_{x} \quad$ velocidade na direção $x$

$\dot{u}_{y} \quad$ velocidade na direção $y$

$\hat{\boldsymbol{u}}$ e $\hat{\boldsymbol{t}}$ deslocamento e tração prescritos

$\tilde{\boldsymbol{e}}_{p} \quad$ estimativa para o função de erro da aproximação $\boldsymbol{u}_{p}$

$\tilde{\mathcal{E}}_{(\mathcal{K})} \quad$ indicador de erro local definido para o elemento $\mathcal{K}$

$\tilde{\mathcal{E}}_{\left(\omega_{j}\right)} \quad$ indicador de erro local definido para a nuvem $\omega_{j}$

$\tilde{u}(\boldsymbol{x}) \quad$ Função aproximadora

$\widehat{\boldsymbol{F}}_{\text {ext }} \quad$ vetor de forças externas generalizadas nos método sem malha

$\widehat{\boldsymbol{F}}_{\text {int }} \quad$ vetor de forças internas generalizadas nos método sem malha

${ }^{t} \widehat{\boldsymbol{F}}_{\text {ext }} \quad$ vetor de forças externas generalizadas nos métodos sem malha no instante $t$

${ }^{t} \widehat{\boldsymbol{F}}_{\text {int }}$ vetor de forças internas generalizadas nos métodos sem malha no instante $t$

${ }^{t} \widehat{\boldsymbol{U}} \quad$ vetor de deslocamentos generalizados nos métodos sem malha no instante $t$

${ }^{t} \boldsymbol{F}_{\text {ext }}$ vetor de forças externas generalizadas no MEFG no instante $t$

${ }^{t} \boldsymbol{F}_{\text {int }} \quad$ vetor de forças internas generalizadas no MEFG no instante $t$

${ }^{t} \boldsymbol{U} \quad$ vetor de deslocamentos generalizados no MEFG no instante $t$

${ }^{x} \boldsymbol{F}_{j}^{\mathcal{K}}$ e ${ }^{y} \boldsymbol{F}_{j}^{\mathcal{K}}$ componentes nodais $j$ nas direções $x$ e $y$ da contribuição do elemento $\mathcal{K}$ para o vetor de forças generalizadas $\boldsymbol{F}$

${ }^{t} \boldsymbol{b}$ forças de volume no passo $t$

${ }^{t} \hat{\boldsymbol{t}} \quad$ tensões de superfície no passo $t$

${ }^{t} \widehat{\boldsymbol{K}}_{\mathrm{sec}}^{\left(i_{t}\right)}$ matriz de rigidez generalizada dos métodos sem malha, na forma secante, no instante de tempo $t$ e iteração $i_{t}$ 
${ }^{t} \widehat{\boldsymbol{K}}_{\mathrm{tg}}^{\left(i_{t}\right)}$ matriz de rigidez generalizada nos métodos sem malha, na forma tangente, no instante de tempo $t$ e iteração $i_{t}$

${ }^{t} \boldsymbol{e}_{p} \quad$ erro da solução ${ }^{t} \boldsymbol{u}_{p}$ ao final do passo $t$

${ }^{t} \boldsymbol{K}_{\text {sec }}^{\left(i_{t}\right)}$ matriz de rigidez generalizada no MEFG, na forma secante, no instante de tempo $t$ e iteração $i_{t}$

${ }^{t} \boldsymbol{u} \quad$ solução exata obtida ao final do passo $t$

${ }^{t} \boldsymbol{u}_{p} \quad$ solução aproximada obtida ao final do passo $t$

${ }^{t} \tilde{\boldsymbol{e}}_{p} \quad$ aproximação para o erro da solução ${ }^{t} \boldsymbol{u}_{p}$ ao final do passo $t$

$\Im_{N}^{k, p} \quad$ família de funções Nuvens- $h p$ formada pela PU $\left\{\phi_{j}(\boldsymbol{x})\right\}_{j=1}^{N}$ geradora do espaço $\mathcal{P}_{k}$ e com capacidade de representar de forma exata polinômios do espaço $\mathcal{P}_{p}$

$\mathcal{F}$ e $\mathcal{G}$ funções auxiliares para o cálculo da lei de evolução do dano no modelo de Mazars

$\mathcal{F}_{T}, \mathcal{F}_{C}, \mathcal{G}_{T}$ e $\mathcal{G}_{C}$ funções auxiliares para o cálculo da lei de evolução do dano no modelo de Mazars para a tração e compressão

$\mathcal{H}$ espaço de Hilbert

$\mathcal{J}_{j} \quad$ conjunto de funções $L_{j i}$

$\mathcal{K} \quad$ elemento finito

$\mathcal{K}^{*} \quad$ elemento vizinho ao elemento $\mathcal{K}$

$\mathcal{N}_{j} \quad$ funções de forma do MEF e empregadas como PU no MEFG

$\mathcal{P}_{k} \quad$ espaço polinomial de grau máximo $k$

$\mathcal{P}_{p} \quad$ espaço polinomial de grau máximo $p$

Q conjunto de pontos nodais

$\mathcal{R}_{x}^{\mathcal{K}}$ e $\mathcal{R}_{y}^{\mathcal{K}}$ resultantes das forças que agem sobre o elemento $\mathcal{K}$, na direção $x$ e $y$ respectivamente

energia de deformação

U energia de deformação

$\mathcal{W}_{\text {ext }}$ trabalho realizado pelas forças externas

$\mathbb{R}^{\eta} \quad$ conjunto dos números reais com dimensão $\eta=1,2$ ou 3

$\Omega \quad$ sub-conjunto aberto de $\mathbb{R}^{\eta}$

$\tilde{U}$ energia de deformação da solução aproximada

$\tilde{x} \quad$ sub-espaço de dimensão finita de $\mathcal{H}$

$\widehat{Q}_{n(\boldsymbol{x})}$ sub-conjunto de $\mathcal{Q}_{N}$ 
$\alpha_{C} \quad$ coeficiente do dano na compressão no modelo de Mazars

$\alpha_{i} \quad$ parâmetro da combinação linear no MMQM

$\alpha_{T} \quad$ coeficiente do dano na tração no modelo de Mazars

$\bar{\varepsilon}_{e q} \quad$ valor não-local da deformação equivalente

$\beta_{1}$ e $\beta_{2}$ parâmetros do material relacionados à formação de deformações residuais no modelo de La Borderie

$\chi \quad$ Energia livre de Gibbs

$\delta S \quad$ área da interseção entre o elemento representativo de volume e qualquer um de seus planos normais a $\vec{n}$

$\delta S_{D} \quad$ parcela de $\delta S$ que não resiste às solicitações internas

$\Delta \tilde{u}^{\left(i_{t}\right)}(\boldsymbol{x})$ acréscimo da função $u$ na iteração $i_{t}$

$\Delta b_{j i}^{\left(i_{t}\right)}$ acréscimo do parâmetro $b_{j i}$ na iteração $i_{t}$

$\Delta t \quad$ passo de tempo

$\Delta u_{j}^{\left(i_{t}\right)}$ acréscimo do parâmetro $u_{j}$ na iteração $i_{t}$

$\delta \quad$ medida de abertura do entalhe

$\Delta \widehat{\boldsymbol{U}}^{\left(i_{t}\right)}$ acréscimo do vetor de deslocamentos generalizados nos métodos sem malha na iteração $i_{t}$

$\delta_{i j} \quad$ Delta de Kronekcer, $\delta_{i j}=1$ se $i=j$ e $\delta_{i j}=0$ se $i \neq j$

$\Delta \boldsymbol{U}^{\left(i_{t}\right)}$ acréscimo do vetor de deslocamentos generalizados na iteração $i_{t}$

$\epsilon \quad$ perturbação dada à matriz $\boldsymbol{K}$ no procedimento de Babuška

$\Gamma \quad$ contorno do domínio $\Omega$

$\Gamma_{N} \quad$ região do contorno em que as condições de Neumman são definidas

$\Gamma_{u} \quad$ região do contorno em que as condições de Dirichlet são definidas

$\kappa \quad$ parâmetro que depende do coeficiente de Poisson e vale $3-4 \nu$ para EPD e $(3-\nu) /(1+$ $\nu)$ para EPT

$\lambda_{1}^{u} \quad$ multiplicador de Lagrange para deslocamentos associado ao nó 1

$\lambda^{\theta} \quad$ multiplicador de Lagrange para as derivadas dos deslocamentos (rotações)

$\lambda_{1}^{\theta} \quad$ multiplicador de Lagrange para rotações associado ao nó 1

$\lambda^{u} \quad$ multiplicador de Lagrange para os deslocamentos

$\lambda_{k} \quad$ multiplicadores de Lagrange

$\mathcal{E}_{\%} \quad$ erro relativo global 
$\nu \quad$ coeficiente de Poisson do material íntegro

$\omega_{j} \quad$ região de influência ou nuvem das aproximações associadas à PU do nó $\boldsymbol{x}_{j}$

$\phi_{j}(\boldsymbol{x})$ função de aproximação associada ao nó $\boldsymbol{x}_{j}$

$\Pi \quad$ energia potencial total

$\psi \quad$ classe de funções que define uma PU

$\rho \quad$ número inteiro que corresponde à quantidade mínima da sobreposição das nuvens

$\rho^{x} \mathrm{e} \rho^{y}$ funções das distâncias em $x$ e em $y$ de cada posição do domínio $\Omega$ com relação a $\left(x_{0}, y_{0}\right)$

$\rho_{j}^{x}$ e $\rho_{j}^{y}$ distâncias em $x$ e em $y$ entre o nó $\boldsymbol{x}_{j}$ e uma posição qualquer $\left(x_{0}, y_{0}\right)$ do domínio $\Omega$

$\sigma_{\text {ef }}$ tensão efetiva

$\sigma_{f} \quad$ tensão de fechamento das micro-fissuras no modelo de La-Borderie

$\theta \quad$ índice de efetividade

$\theta_{\mathcal{K}} \quad$ índice de efetividade para o elemento finito $\mathcal{K}$

$v \quad$ parâmetro que controla a velocidade do refinamento no processo adaptativo

$\varepsilon_{i} \quad$ deformação principal $i$

$\varepsilon_{d 0} \quad$ deformação equivalente limite para o material íntegro

$\varepsilon_{d} \quad$ deformação equivalente limite para a evolução do dano nos pontos já danificados

$\varepsilon_{e q} \quad$ deformação equivalente

$\left(\boldsymbol{\Phi}_{p+1}^{0}\right)_{\omega_{j}}^{T}$ funções de aproximação da função indicadora de erro $\tilde{\boldsymbol{e}}_{p}$ associadas à nuvem $\omega_{j}$

$\boldsymbol{\alpha} \quad$ vetor contendo os parâmetros $\alpha_{i}$

$\mathbf{\Phi} \quad$ vetor das funções de aproximação

$\phi_{j} \quad$ vetor das funções de forma associadas ao nó $\boldsymbol{x}_{j}$

$\boldsymbol{\psi}_{j} \quad$ vetor das funções resultantes do enriquecimento de $\mathcal{N}_{j}$

$\boldsymbol{\sigma} \stackrel{\text { def }}{=}\left\{\begin{array}{ccc}\sigma_{x} & \sigma_{y} & \sigma_{x y}\end{array}\right\}$ vetor de tensões definido para problemas de estado plano

$\boldsymbol{\sigma}^{\mathrm{tg}}$ forma compacta do tensor de tensões obtida pela forma tangente da relação constitutiva

$\Theta^{\mathcal{K}} \quad$ vetor de forças nodais generalizadas de correção para o equilíbrio

$\boldsymbol{\theta}^{\mathcal{K}} \quad$ tensões de correção do equilíbrio para o elemento $\mathcal{K}$

$\varepsilon \stackrel{\text { def }}{=}\left\{\begin{array}{llll}\varepsilon_{x} & \varepsilon_{y} & \gamma_{x y}\end{array}\right\}$ vetor de deformações definido para problemas de estado plano

$\varepsilon^{e}$ e $\varepsilon^{a}$ parcela de deformações elástica e anelástica

$\dot{\varepsilon} \quad$ vetor das taxas de variação das deformações principais com o tempo 
$\bar{\Pi} \quad$ funcional modificado de $\Pi$ com a introdução dos multiplicadores de Lagrange

$\boldsymbol{\Sigma} \quad$ tensor de tensões

$\bar{\Omega} \quad$ domínio fechado e limitado em $\mathbb{R}^{\eta}$

$\ddot{\lambda}^{u_{x}}$ e $\ddot{\lambda}^{u_{y}}$ vetor dos parâmetros nodais dos multiplicadores de Lagrange para os deslocamentos $u_{x}$ e $u_{y}$

$\dot{\lambda}^{u_{x}}$ e $\dot{\lambda}^{u_{y}}$ vetor dos parâmetros nodais da segunda taxa de variação no tempo dos multiplicadores de Lagrange

$\dot{\sigma} \quad$ taxas do tensor de tensões

$\dot{\varepsilon} \quad$ taxas do tensor de deformações

$\hat{\boldsymbol{\Theta}}_{j}^{\mathcal{K}} \quad$ vetor com os valores de $\hat{\boldsymbol{\Theta}}^{\mathcal{K}}$ associados ao nó $\boldsymbol{x}_{j}$

$\mathcal{M}$ momento das forças atuantes sobre o elemento $\mathcal{K}$

$\partial \Omega \quad$ contorno suave de $\Omega$

$\tilde{\mathcal{E}}_{\%} \quad$ erro relativo estimado global

${ }^{\alpha} \boldsymbol{\theta}^{\mathcal{K}}$ tensões $\boldsymbol{\theta}^{\mathcal{K}}$ na face $\alpha$ do elemento $\mathcal{K}$

${ }^{\alpha} \boldsymbol{\theta}_{j}^{\mathcal{K}} \quad$ valor que ${ }^{\alpha} \boldsymbol{\theta}^{\mathcal{K}}$ assume no ponto nodal $\boldsymbol{x}_{j}$

${ }^{a} \hat{\boldsymbol{\Theta}}_{j}^{\mathcal{K}}$ parcela de $\hat{\boldsymbol{\Theta}}_{j}^{\mathcal{K}}$ atuante sobre a face $a$ do elemento $\mathcal{K}$

\section{Operadores}

$(\cdot) \quad$ produto interno

$[\boldsymbol{t}(\bullet)]$ operador do salto das tensões calculadas na face comum a dois elementos $\mathcal{K}$ e $\mathcal{K} *$ onde se tem o campo de deslocamento aproximado por

$\overline{\mathcal{B}}(\bullet, \bullet)$ forma modificada de $\mathcal{B}(\bullet, \bullet)$ após a aplicação do método dos Multiplicadores de Lagrange

$\bar{l}(\cdot) \quad$ forma modificada de $l(\cdot)$ após a aplicação do método dos Multiplicadores de Lagrange

$\boldsymbol{a} \otimes \boldsymbol{b}$ notação para o produto diádico ou tensorial, produzindo o tensor $T_{i j}=a_{i} b_{j}$

$\boldsymbol{B} \quad \boldsymbol{L} \boldsymbol{\Phi}^{T}$, operador de transformação: deslocamento generalizado-deformação

$\boldsymbol{B}_{p} \quad$ operador $\boldsymbol{L} \boldsymbol{\Phi}_{p}^{T}$

$\boldsymbol{B}_{p+1}^{0} \quad$ operador $\boldsymbol{L}\left(\boldsymbol{\Phi}_{p+1}^{0}\right)^{T}$

$\boldsymbol{L} \quad$ matriz de operadores diferenciais que determina o campo de deformações a partir dos deslocamentos

$\boldsymbol{\nabla} \quad\left[\begin{array}{ll}\frac{\partial}{\partial x_{1}} & \frac{\partial}{\partial x_{2}}\end{array}\right]^{T}$ 
$\langle\bullet, \star, \cdots\rangle$ representa o espaço gerado pelas funções $\bullet, \star, \cdots$

$\Lambda(\bullet, \star)$ funcional através do qual as condições de contorno essenciais são impostas no Método dos Multiplicadores de Lagrange

$\langle\boldsymbol{t}(\bullet)\rangle_{m}$ operador da média das tensões calculadas na face comum a dois elementos $\mathcal{K}$ e $\mathcal{K} *$ onde se tem o campo de deslocamento aproximado por $\bullet$ e $\bullet *$

$\langle\bullet\rangle_{+} \quad$ parte positiva de $(\bullet)$

$\langle\bullet\rangle_{-} \quad$ parte negativa de $(\bullet)$

$\|\boldsymbol{w}\|_{u}$ norma energia definida para funções $\boldsymbol{w} \in \mathcal{H}^{1}$

$\|\bullet\|_{L_{2}}$ norma $L_{2}$

$\|\bullet\|_{L_{\infty}(\Omega)}=\max |\bullet|$ norma uniforme ou de Chebyshev

$\|\bullet\|_{u}$ norma energia de $\bullet$

$\|u\|_{L_{p}(\Omega)}$ norma em $L^{p}$

$|u|_{H^{m}(\Omega)}$ semi-norma no espaço $\mathcal{H}^{m}$

$\mathcal{B}(\bullet, \bullet)$ forma bi-linear em $\mathcal{H} \times \mathcal{H} \rightarrow \mathbb{R}$

$\mathcal{B}_{\mathcal{K}}^{t g}(\bullet, \bullet)$ parcela de contribuição no elemento $\mathcal{K}$ para $\mathcal{B}^{t g}(\bullet, \bullet)$

$\mathcal{B}_{\mathcal{K}}(\bullet, \bullet)$ parcela de contribuição no elemento $\mathcal{K}$ para $\mathcal{B}(\bullet$

$\mathcal{L}_{\mathcal{K}}(\bullet)$ operador linear com os dados para a aproximação de Galerkin da forma variacional do PVC do erro em cada elemento

$\max (\bullet, \star, \ldots)$ operador que retorna o maior valor de $\bullet$ entre os $m$ existentes

$\min (\bullet, \star, \ldots)$ função que retorna o menor valor entre $\bullet, \star, \ldots$

$\backslash$ operador de exclusão; $\Psi \backslash \Upsilon$ é o mesmo que $\Psi-(\Psi \cap \Upsilon)$

$\stackrel{\text { def }}{=}$ é definido como

span espaço gerado por

$\left\{B_{k} \cdot\right\}_{k=0}^{m-1}$ operadores lineares diferenciais sobre o contorno

$A \bullet \quad$ operador linear diferencial de ordem $2 m$

$l(\bullet) \quad$ forma linear em $\mathcal{V} \rightarrow \mathbb{R}^{1}$

$\operatorname{Tr}(\bullet)$ traço de um tensor

\section{Abreviaturas}

DEM Difuse Element Method

EFGM Element Free Galerkin Method 
EPD Estado plano de deformação

EPT Estado plano de tensão

FPM Finite Point Method

GFEM Generalized Finite Element Method

MDC Mecânica do Dano Contínuo

MED Método dos Elementos Difusos

MEFG Método dos Elementos Finitos Generalizados

MEFPU Método dos Elementos Finitos da Partição da Unidade

MGLE Método de Galerkin Livre de Elementos

MMQ Método dos Mínimos Quadrados

MMQF Método dos Mínimos Quadrados Fixos

MMQM Método dos Mínimos Quadrados Móveis

MPF Método dos Pontos Finitos

MRE Método dos Resíduos em Elementos

$\mathrm{MRE}_{p+1, p+2}$ Método do Resíduo em Elementos em que o erro é aproximado em $\boldsymbol{X}_{p+1, p+2}^{0}$

$\mathrm{MRE}_{p+1}$ Método do Resíduo em Elementos em que o erro é aproximado em $\boldsymbol{X}_{p+1}^{0}$

MREP Método de Recuperação pelo Equilíbrio das Parcelas

MRS Método dos Resíduos em Sub-domínios

NG número de pontos de integração, seja na quadratura de Gauss-Legendre, seja na de Gauss-Lobatto

NGL número de graus de liberdade

PUFEM Partition of Unity Finite Element Method

PVC Problemas de Valor de Contorno

RKPM Reproducing Kernel Particle Method

SPH Smoothed Particle Hydrodynamics Method 


\section{Capítulo 1}

\section{Introdução}

\subsection{Considerações Iniciais}

Neste trabalho, são apresentados resultados e conclusões de uma pesquisa realizada sobre certas categorias de métodos numéricos, os sem malha e o dos elementos finitos generalizados, aplicados a problemas com não-linearidade física.

A pesquisa foi desenvolvida segundo duas linhas: uma de caráter numérico, relacionada ao desenvolvimento dos métodos e a outra voltada às aplicações na mecânica dos materiais, mais precisamente, na mecânica do dano contínuo. Essas duas linhas, em princípio independentes, combinam-se quanto à aplicação na simulação dos efeitos da formação e propagação de descontinuidades em estruturas.

Procura-se mostrar, ao longo do texto, como as alternativas numéricas, protagonistas do estudo realizado, podem ser melhor exploradas para fins de análise estrutural, especialmente em problemas cujo o comportamento não-linear é governado pela evolução do dano contínuo. O principal objetivo, contudo, é demonstrar a viabilidade do uso dos métodos mencionados para a solução de problemas com propagação de descontinuidades, salientando-se não apenas suas vantagens como também algumas deficiências e como contorná-las. O trabalho desenvolvido não se conclui em si mesmo mas, ao contrário, representa os primeiros passos na direção de se obter uma ferramenta numérica especialmente talhada para aplicações no campo da propagação de defeitos. O emprego de aproximações de simples implementação, mas eficientes para o tipo de problema estudado, a caracterização de particularidades dos métodos segundo a análise realizada, a definição de medidas de erro para se avaliar a qualidade da solução e posterior adaptatividade, são algumas das questões para as quais se buscam respostas 
neste trabalho. As respostas obtidas, se por um lado ajudam na melhor compreensão dos fenômenos físicos simulados, bem como trazem contribuições para as formulações utilizadas, por outro lado não são inteiramente conclusivas permanecendo abertas para futuros desenvolvimentos.

\subsubsection{Quanto aos Métodos Numéricos: Sem Malha e dos Elemen- tos Finitos Generalizados}

Métodos sem malha, Meshless, podem ser entendidos, de acordo com o trabalho de DUARTE (1995), como métodos numéricos para a solução de problemas de valor de contorno, PVC, cujas equações básicas de governo do modelo discreto independem, ou quase, da definição de uma malha de elementos finitos. Em resumo, a solução aproximada do problema, em um espaço de dimensão finita, é construída sem que a conectividade entre os pontos nodais desta aproximação seja pré-estabelecida.

No método dos elementos finitos, MEF, a aproximação é definida mediante interpolações locais em cada elemento, sendo, por isto, válida apenas nesse sub-domínio. A continuidade da aproximação fica garantida com o pré-estabelecimento de uma conectividade entre os nós. Já nos procedimentos sem malha, dada a ausência dos elementos, a aproximação é construída em cada posição do domínio global, sendo, portanto, definida de modo dinâmico; mantém-se, entretanto, seu caráter local conforme a definição de domínios de influência, denominados nuvens em DUARTE; ODEN (1995). Tal estratégia admite que, em cada posição de interesse, novas relações de conectividade nodal sejam estabelecidas, possibilitando com que a aproximação do domínio seja feita sem a necessidade de se definir elementos, mas apenas a partir de uma distribuição de pontos nodais.

Diversas são as variações dos Métodos Sem Malha, podendo ser reunidas da seguinte maneira:

- Hidrodinâmica de Partículas Suavizado (Smoothed Particle Hydrodynamics SPH), de MONAGHAN (1982), (1994);

- Métodos baseados diretamente nas aproximações de mínimos quadrados móveis:

- Método dos Elementos Difusos, MED (Difuse Element Method - DEM), introduzido em NAYROLES; TOUZOT; VILLON (1992); 
- Método de Galerkin Livre de Elementos, MGLE (Element Free Galerkin Method - EFGM), BELYTSCHKO; LU; GU (1994);

- Método dos Pontos Finitos, MPF (Finite Point Method - FPM), desenvolvido em OÑATE; IDELSOHN; ZIENKIEWICZ (1995), OÑATE (1996a) e OÑATE (1996b)

- Método das Partículas Reprodutoras do Núcleo (Reproducing Kernel Particle Method - RKPM), de W. K. LIU; ZHANG (1995); LIU (1995);

- Métodos baseados na definição da Partição da Unidade:

- Método das Nuvens $h p$ (hp-Clouds Method), de DUARTE; ODEN (1995); DUARTE (1996); DUARTE; ODEN (1996a), (1996b);

- Método dos Elementos Finitos Partição da Unidade, MEFPU (Partition of Unity Finite Element Method - PUFEM), descrito em MELENK (1995), MELENK; BABUŠKA (1996) e BABUŠKA; MELENK (1997);

- Método de Galerkin das Ondas Pequenas (Wavelet Galerkin Method), proposto por AMARATUGA; WILLIAMS (1997);

Não é objetivo deste trabalho apresentar uma revisão de todos esses métodos e, por isso, apenas o MGLE e o Método das Nuvens $h p$, utilizados para as experimentações numéricas, são discutidos a seguir. Para maiores detalhes sobre os métodos sem malha, além dos artigos já citados, podem ser incluídas as revisões realizadas em DUARTE (1995) e BELYTSCHKO (1996).

No MGLE, utiliza-se, para a obtenção das funções de forma, as funções de aproximação do Método dos Mínimos Quadrados Móveis, MMQM, de acordo com o trabalho de LANCASTER; SALKAUSKAS (1981). Os elementos essenciais desse procedimento são uma base de funções geralmente polinomiais, funções de ponderação, e uma distribuição de pontos. As constantes que aparecem na função aproximadora são determinadas impondo-se a minimização do erro entre a função aproximadora e a solução exata. Caso seja necessário que se enriqueça a aproximação, monômios de ordem mais alta são adicionados à base de funções, o que, na maioria das vezes, deve ser acompanhado pela introdução de novos pontos nodais à discretização.

No Método das Nuvens $h p$, por sua vez, procura-se generalizar as idéias desenvolvidas no MGLE, reconhecendo-se que as funções de forma devam constituir uma Partição da Unidade, PU, ODEN; REDDY (1976), podendo ser enriquecidas sem que a 
base de funções dos MMQM seja alterada, ou seja, sem a necessidade de novos pontos nodais.

Para que um conjunto de funções constitua uma PU, é necessário que a soma de seus valores seja igual à unidade em cada posição do domínio. Entre as funções que compartilham dessa propriedade estão aquelas obtidas via MMQM e, entre elas, as Funções de Shepard, SHEPARD (1968); também pertencem a essa categoria várias das funções de forma empregadas no MEF. As funções de Shepard, apesar de gerarem aproximações pobres, são particularmente interessantes ao serem utilizadas para a construção das funções de aproximação pelo Método das Nuvens $h p$.

O enriquecimento da aproximação na formulação do Método das Nuvens $h p$ é realizado a partir da multiplicação das funções de PU por um conjunto de funções linearmente independentes. Não há, portanto, a necessidade de se introduzir novos pontos ao domínio. A estratégia de enriquecimento torna o Método das Nuvens $h p$ vantajoso ao ser aplicado em procedimentos adaptativos do tipo $p$, bem como em problemas cuja solução apresente elevados gradientes.

Cabe, ainda, ressaltar que ambos os métodos comentados originam-se de uma mesma formulação, proposta no MED. Por essa razão, seria mais correto denominálos como processos. Sua qualificação como método, entretanto, é avalizada pelo uso corrente na literatura e, dessa maneira, também será adotada neste texto.

Ainda que não se encaixe exatamente na definição de método sem malha, o Método dos Elementos Finitos Generalizados, MEFG, proposto como uma formulação híbrida do MEF em ODEN; DUARTE; ZIENKIEWICZ (1998) e fundamentado em STROUBOULIS; BABUŠKA; COPPS (2000), até poderia ser mencionado dentro desse contexto. Utilizando-se de conceitos do MEFPU e do Método das Nuvens $h p$, no MEFG, estabelece-se uma malha que serve apenas para se definir uma partição da unidade, sobre a qual é realizado o enriquecimento das funções de forma, responsável pela qualidade do método. A aproximação é construída em uma formulação que minimiza a importância da malha de elementos finitos. Empregando estratégias dos métodos sem malha dentro da estrutura do MEF, mais simples e tradicionalmente mais difundido, o MEFG pode também ser entendido como uma "ponte" entre estas diversas abordagens. Permite, desse modo, uma maior flexibilidade na resolução numérica de PVC, viabilizando a introdução de diferentes especializações de implementação conforme seja mais interessante em cada caso de aplicação. 


\subsubsection{Quanto à Mecânica dos Materiais}

Um dos pontos de interesse deste trabalho refere-se à aplicação dos métodos sem malha, incluindo-se o MEFG, na simulação do comportamento não-linear de estruturas decorrente da evolução de um processo de plastificação e microfissuração do material. Nesse sentido, deve ser considerado um importante conceito, o dano, KRAJCINOVIC; LEMAITRE (1987) e PROENÇA (1991).

O dano de materiais é aqui entendido como o resultado de um processo de evolução de micro-defeitos e fissuras que, no limite, conduz à ruptura local do elemento de "volume representativo", em torno de um ponto, LEMAITRE; CHABOCHE (1990). Em uma análise fenomenológica, a evolução do dano produz perdas de resistência e de rigidez do material. No caso do concreto, por exemplo, a formação e o crescimento de microfissuras têm influência direta sobre a resposta mecânica. Já no caso de metais, o processo de danificação é precedido pela plastificação, e reduz, significativamente, a ductilidade do material.

A fratura discreta pode ser entendida como resultante da localização do dano evolutivo em uma certa região do corpo. A Mecânica do Dano Contínuo, MDC, diz respeito, portanto, a fenômenos que ocorrem em uma fase anterior àqueles descritos pela Mecânica da Fratura. Na MDC, lida-se com modelos constitutivos aptos a descrever o comportamento dos materiais penalizados pela danificação, mas ainda considerados como meios contínuos. Na Mecânica da Fratura, trata-se das condições de propagação de uma ou mais descontinuidades, as trincas, em um meio contínuo. As formulações sobre a localização de deformações, segundo as propostas de OLIVER (1995) e MANZOLI; OLIVER; CERVERA (1998), procuram estabelecer uma transição entre as duas abordagens.

Em uma análise tradicional de propagação de fratura, via MEF, é necessária uma série de artifícios numéricos, em particular a redefinição da malha, para que as singularidades presentes sejam simuladas de forma adequada. Exemplos desses procedimentos podem ser encontrados em ORTIZ; LEROY; NEEDLEMAN (1987); DVORKIN; CUITIÑO; GIOIA (1990) e OLIVER (1995). Por outro lado, a flexibilidade no enriquecimento das aproximações, característica do método das nuvens e do MEFG, permite com que se vislumbre um campo fértil para sua aplicação naquele tipo de problema. Envolvendo a aplicação do Método das Nuvens $h p$ e do MGLE, diversos trabalhos já foram propostos no âmbito da análise de fratura, HEGEN (1997), ODEN; DUARTE (1997a) e BELYTSCHKO; LU; GU (1993) e de outros tipos de sin- 
gularidade, como em CORDES; MORAN (1996), KRYSL; BELYSTSCHKO (1997), ODEN; DUARTE (1997b) e KRONGAUZ; BELYTSCHKO (1998). Com o MEFG, podem ser destacados: DUARTE (2001) e, sob a denominação de MEFX (Método dos Elementos Finitos Extendidos), encontram-se BELYTSCHKO; BLACK (1999), MOËS; DOLBOW; BELYTSCHKO (1999), DOLBOW (1999) e, aplicado de modo específico a placas, DOLBOW; MOËS; BELYTSCHKO (2000).

Já quanto ao processo de danificação do meio, não foram encontradas na bibliografia disponível, aplicações do Método das Nuvens, do MGLE e do MEFG. É, portanto, nesse contexto que se insere a presente pesquisa: procura-se oferecer contribuições ao estudo da viabilidade de novas abordagens numéricas, especialmente do MEFG, para a simulação do comportamento não-linear de estruturas decorrente do fenômeno de formação e propagação de descontinuidades.

\subsection{Organização do Texto}

No capítulo 2, as formulações do MGLE, Método das Nuvens e MEFG são apresentadas, buscando-se mostrar a relação que existe entre esses métodos. A ordem em que são introduzidos é proposital, não apenas para respeitar a seqüência cronológica em que surgiram na literatura científica, mas também como uma maneira de ilustrar a sua evolução no sentido do MEFG.

Experimentações com esses três métodos em problemas de análise linear são reunidas no capítulo 3. O MGLE e o Método das Nuvens são aplicados para a solução de um problema de associação plana entre estruturas, painéis de edifícios altos simulados pela técnica do meio contínuo. Utiliza-se deste exemplo para se discutir a convergência dos métodos e a influência dos parâmetros utilizados. O MEFG, por sua vez, é empregado em problemas de elasticidade bi-dimensional. Nos problemas selecionados, a resposta numérica é averiguada com relação ao tipo de malha, convergência da solução, travamento de Poisson e presença de singularidades. Em todos estes exemplos, procura-se mostrar vantagens conferidas pela estratégia de enriquecimento, polinomial ou não.

O conceito de dano é discutido no capítulo 4, apresentando-se também algumas definições da Mecânica do Dano Contínuo. Em seguida, os dois modelos adotados para as análise não-lineares são descritos resumidamente. Ao final, o emprego de uma abordagem não-local é justificada como uma maneira de garantir a aplicação dos 
modelos constitutivos de dano contínuo.

No capítulo 5, são apresentadas novas experimentações numéricas, agora em análise não-linear. Um problema de uma viga de concreto armado é, primeiramente, analisado com o Método das Nuvens $h p$, considerando-se a teoria de vigas de Bernoulli. Resultados de análises estática e dinâmica, para os modelos de dano adotados, são utilizados para ilustrar a aplicação do método. Algumas conclusões sobre o comportamento do material e a viabilidade do emprego dos modelos são salientadas. O mesmo problema é aproximado pelo MEFG e os resultados da análise estática com discretização bi-dimensional são utilizados para chamar atenção sobre alguns detalhes de sua implementação e justificar a necessidade de um procedimento adaptativo de solução.

Adaptatividade e estimativa de erro são os assuntos do capítulo 6. Uma pequena revisão a esse respeito é realizada com o objetivo de conduzir o leitor até a introdução de uma técnica de análise adaptativa para o MEFG. O Método dos Resíduos em Elementos Equilibrados é descrito segundo a abordagem de enriquecimento da PU e dois exemplos são apresentados. Em seguida, o estimador de erro é discutido em análise não-linear. Um algoritmo adaptativo é introduzido e considerações sobre transferência de variáveis na formulação do MEFG são realizadas. O capítulo se encerra com dois exemplos relativos a problemas não-lineares. O primeiro deles é convenientemente escolhido para assegurar hipóteses admitidas na discussão sobre o estimador em análise não-linear, sendo utilizado para avaliar as medidas de erro ao final de cada passo de carregamento da estrutura. O segundo e último exemplo, bem mais complexo, é resolvido adaptativamente, comprovando-se a viabilidade das medidas de erro estabelecidas e do algoritmo de erro adotado.

O capítulo 7 reúne as considerações finais e conclusões obtidas durante esta pesquisa, embasadas nos resultados e observações apresentados ao longo do texto. Dessa discussão originam-se propostas para trabalhos futuros, visando o aperfeiçoamento da técnica numérica empregada para análise da propagação de defeitos em meio contínuo.

Ao final da tese, alguns tópicos de assuntos diversos são reunidos como apêndices. Sua leitura, contudo, não é essencial para o entendimento do texto, podendo ser consultados para o esclarecimentos de detalhes relacionados com a formulação e implementação dos métodos numéricos estudados. 


\section{Capítulo 2}

\section{Fundamentos dos Métodos Numéricos}

Várias são as razões para dedicar um capítulo à descrição dos métodos numéricos discutidos neste trabalho e, entre elas, duas merecem ser destacadas. A primeira delas, e a mais óbvia, é a oportunidade de registrar toda uma revisão bibliográfica realizada sobre um tema relativamente recente e que vem atraindo considerável atenção no meio científico. Com esse intuito, procura-se completar as discussões iniciadas no capítulo 1 apresentando, dentro de um certo formalismo, fundamentos dos métodos empregados neste trabalho, o MGLE, o Método das Nuvens $h p$ e o MEFG. A segunda razão para a existência deste capítulo consiste em apresentar bases teóricas para os desenvolvimentos descritos ao longo do texto; introduzindo-as sob um enfoque em que o MEFG é interpretado como uma particularização, à estrutura do MEF, de conceitos oriundos das formulações sem malha.

O primeiro assunto apresentado refere-se ao MMQM, entendido como uma técnica de construção dinâmica de aproximações. Em seguida, a família de funções denominada genericamente por Nuvens- $h p$ é discutida como uma generalização das funções do MMQM em que o enriquecimento da aproximação é realizado aplicando-se o conceito de PU. O MGLE e o Método das Nuvens $h p$ são introduzidos como sistematizações das aplicações dessas estratégias na aproximação de Galerkin para o PVC. Ao final, o MEFG é introduzido como uma particularização do Método das Nuvens $h p$, estabelecendo a "ponte", já citada no capítulo 1, entre as abordagens sem malha e finitos convencional. 


\subsection{Método dos Mínimos Quadrados Móveis (MMQM)}

O Método dos Mínimos Quadrados Móveis, MMQM, é um método de aproximação numérica. Através dele, procura-se encontrar uma função que melhor se ajuste a um conjunto de dados, associados a pontos nodais. Este procedimento foi introduzido em LANCASTER; SALKAUSKAS (1981), como uma variação do já bastante difundido Método dos Mínimos Quadrados, MMQ, RIVLIN (1969). Basicamente, a diferença entre esses dois métodos está na utilização de uma função de ponderação que, no MMQM, acompanha o ponto onde se deseja definir a aproximação.

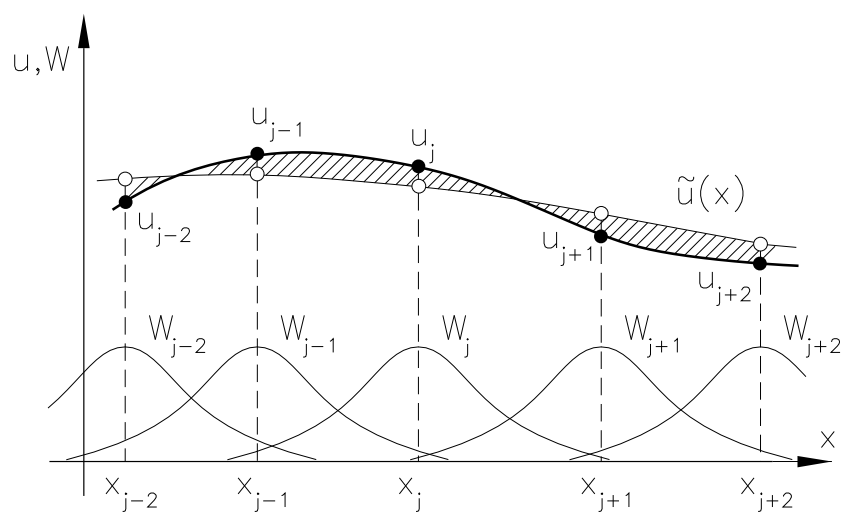

Figura 2.1: Método dos Mínimos Quadrados Móveis

Para melhor entender o MMQM, considera-se o problema de uma função contínua $u(\boldsymbol{x}): \bar{\Omega} \rightarrow \mathbb{R}^{\eta},(\eta=1)$, que deve ser aproximada conhecendo-se apenas seus valores $u_{j}$ em um conjunto de pontos nodais $Q_{N}=\left\{\boldsymbol{x}_{j}\right\}_{j=1}^{N}, \boldsymbol{x}_{j} \in \bar{\Omega}$. Na Figura 2.1 esse problema é ilustrado como um ajuste de curvas em campo uni-dimensional; em uma descrição geral, vale, contudo, $\bar{\Omega} \mathrm{em} \mathbb{R}^{\eta}$, para $\eta=1,2$ ou 3 .

Em cada posição $\boldsymbol{x}$ do domínio, uma aproximação local, $\tilde{u}(\boldsymbol{x})$, deve ser definida empregando-se um sub-conjunto de $n(\boldsymbol{x}) \leq N$ pontos vizinhos $\widehat{Q}_{n(\boldsymbol{x})} \subset \mathcal{Q}_{N}$. Tal aproximação pode ser expressa na forma de uma combinação linear de uma base de funções $\boldsymbol{P}=\left\{p_{i}\right\}_{i=1}^{m},(m \leq n(\boldsymbol{x}))$, segundo os parâmetros $\alpha_{i}$ :

$$
u(\boldsymbol{x}) \approx \tilde{u}(\boldsymbol{x})=\sum_{i=1}^{m} p_{i}(\boldsymbol{x}) \alpha_{i}(\boldsymbol{x})=\boldsymbol{p}^{T}(\boldsymbol{x}) \boldsymbol{\alpha}(\boldsymbol{x})
$$

A menos de aplicações específicas, emprega-se uma base de monômios, $\boldsymbol{P}_{k}$, geradora de um espaço de polinômios completos até o grau $k$.

Seja, também, $W_{j}$ uma função peso que assume valores não-nulos apenas na vizinhança do ponto $\boldsymbol{x}_{j}$. Tal função apresenta, portanto, o suporte compacto, ou seja, 
$W_{j} \in C_{0}^{l}\left(\omega_{j}\right)$, onde $l$ representa a continuidade de $W_{j}, \omega_{j}$ a vizinhança de $\boldsymbol{x}_{j}$ em que a função peso é definida e o zero indica que a função tem valor não-nulo apenas no interior de $\omega_{j}$. A vizinhança é denominada suporte, região de influência ou mesmo nuvem do ponto $\boldsymbol{x}_{j}$, sendo limitada por uma medida de referência ${ }^{1} R_{j}$ e representada por $\omega_{j}=\left\{\boldsymbol{x} \in \Omega ;\left\|\boldsymbol{x}-\boldsymbol{x}_{j}\right\| \leq R_{j}\right\}$, Figura 2.2.

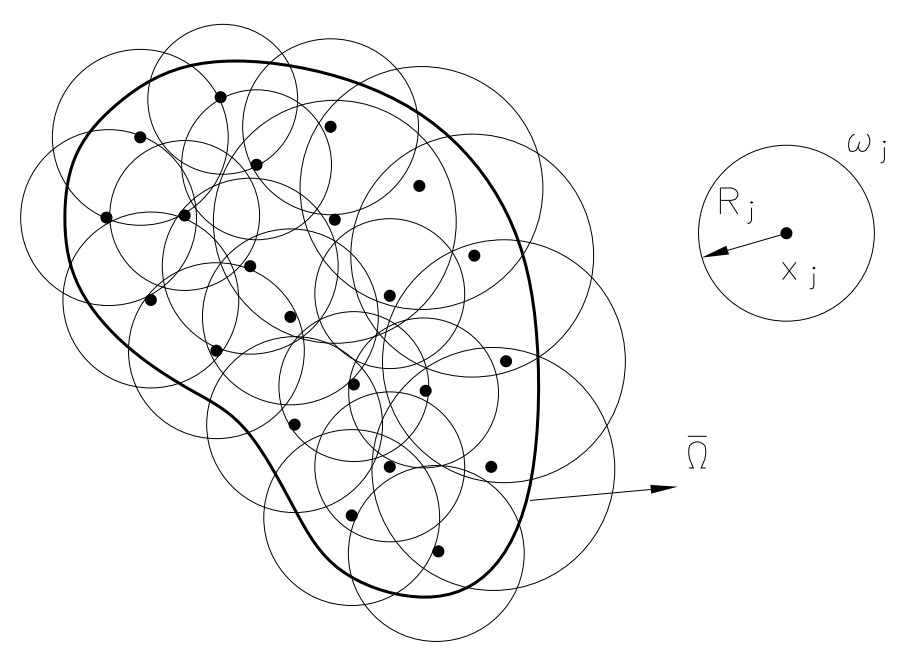

Figura 2.2: Representação das nuvens em $\mathbb{R}^{2}$

Na abordagem do MMQM, os coeficientes $\boldsymbol{\alpha}(\boldsymbol{x})$ são determinados minimizandose uma função $J(\boldsymbol{x})$ que reúne as distâncias entre $\tilde{u}(\boldsymbol{x})$ e $u(\boldsymbol{x})$, ponderadas pelas funções $W_{j}$.

$$
J(\boldsymbol{x})=\sum_{j=1}^{n(\boldsymbol{x})} W_{j}\left(\boldsymbol{x}-\boldsymbol{x}_{j}\right)\left\{u_{j}-\tilde{u}\left(\boldsymbol{x}_{j}\right)\right\}^{2}
$$

Observa-se que apenas os $n(\boldsymbol{x})$ pontos nodais $x_{j}$, cuja região de influência $\omega_{j}$ contenha a posição $\boldsymbol{x}$, participam da somatória acima. A relação que determina os coeficientes $\boldsymbol{\alpha}(\boldsymbol{x})$ resulta:

$$
\boldsymbol{\alpha}(\boldsymbol{x})=\sum_{j=1}^{n(\boldsymbol{x})} \boldsymbol{A}^{-1}(\boldsymbol{x}) \boldsymbol{B}_{j}(\boldsymbol{x}) u_{j}
$$

onde:

$$
\boldsymbol{A}(\boldsymbol{x})=\sum_{r=1}^{n(\boldsymbol{x})} W_{r}\left(\boldsymbol{x}-\boldsymbol{x}_{r}\right) \boldsymbol{p}\left(\boldsymbol{x}_{r}\right) \boldsymbol{p}^{T}\left(\boldsymbol{x}_{r}\right)
$$

\footnotetext{
${ }^{1} R_{j}$ é um parâmetro de extrema importância, pois é responsável pelo caráter local da aproximação. Quanto maior $R_{j}$, maior o número de pontos nodais cujos valores de $u_{j}$ contribuem para se construir a aproximação.
} 


$$
\boldsymbol{B}_{j}(\boldsymbol{x})=W_{j}\left(\boldsymbol{x}-\boldsymbol{x}_{j}\right) \boldsymbol{p}\left(\boldsymbol{x}_{j}\right)
$$

Dessa maneira, a aproximação (2.1), passa a ser escrita como:

$$
u(\boldsymbol{x}) \approx \tilde{u}(\boldsymbol{x})=\sum_{j=1}^{n(\boldsymbol{x})} \phi_{j}(\boldsymbol{x}) u_{j}=\boldsymbol{\Phi}^{T} \boldsymbol{U}
$$

sendo $\phi_{j}$ um elemento genérico da base de funções de aproximação, com o mesmo suporte $\omega_{j}$ das funções peso, e dado por:

$$
\phi_{j}(\boldsymbol{x})=\boldsymbol{p}^{T}(\boldsymbol{x}) \boldsymbol{A}^{-1}(\boldsymbol{x}) \boldsymbol{B}_{j}(\boldsymbol{x})
$$

Nota-se que, para a representação vetorial, são definidos os seguintes vetores de parâmetros nodais e de funções de forma:

$$
\begin{aligned}
& \boldsymbol{U}^{T} \stackrel{\text { def }}{=}\left[\begin{array}{llll}
u_{1} & u_{2} & \cdots & u_{N}
\end{array}\right] \\
& \boldsymbol{\Phi}^{T} \stackrel{\text { def }}{=}\left[\begin{array}{llll}
\phi_{1} & \phi_{2} & \cdots & \phi_{N}
\end{array}\right]
\end{aligned}
$$

A existência da inversa de $\boldsymbol{A}(\boldsymbol{x})$ depende da conveniente definição dos $R_{j}$ de modo que respeite a condição $n(\boldsymbol{x}) \geq m$. Tal condição é necessária mas não suficiente, DUARTE (1996), e, de certo modo, acaba por restringir a liberdade de definição da distribuição nodal. Por sua vez, o enriquecimento polinomial da aproximação pode ser obtido aumentando-se o grau máximo do espaço de polinômios gerado pela base $\boldsymbol{P}_{k}$ com a introdução de novos termos. Este procedimento, entretanto, produz matrizes $\boldsymbol{A}(\boldsymbol{x})$ de elevada ordem encarecendo computacionalmente a técnica de aproximação.

A forma e o tipo das funções de ponderação $W_{j}$ têm grande influência na construção da aproximação, sendo diretamente responsáveis, em combinação com a base $\boldsymbol{P}$, pela sua continuidade. Pode-se demonstrar que:

$$
\tilde{u}(\boldsymbol{x}) \in C_{0}^{\min (k, l)}\left(\omega_{j}\right) \text { se }\left\{\begin{array}{c}
\boldsymbol{P} \in C^{k}\left(\omega_{j}\right) \\
W_{j}(\boldsymbol{x}) \in C_{0}^{l}\left(\omega_{j}\right)
\end{array}\right.
$$

Uma discussão ampla sobre as propriedades das aproximações do MMQM, bem como estudos sobre a influência da forma e continuidade das funções de ponderação, podem ser encontrados, por exemplo, em BELYTSCHKO; LU; GU (1994) bem como em MENDONÇA; BARCELLOS; DUARTE (2000). 
Por último, considera-se o caso específico de se ter uma base de funções $\boldsymbol{P}_{0}=$ $\{1\}$. Das definições de $\boldsymbol{A}(\boldsymbol{x}),(2.4)$, e $\boldsymbol{B}_{j}(\boldsymbol{x}),(2.5)$, resultam:

$$
\begin{gathered}
\boldsymbol{A}(x)=\sum_{r=1}^{n(\boldsymbol{x})} W_{r}\left(x-x_{r}\right)\{1\}[1]=\sum_{r=1}^{n(\boldsymbol{x})} W_{r}\left(x-x_{r}\right) \\
\boldsymbol{B}_{j}(x)=W_{j}\left(x-x_{j}\right)\{1\}=W_{j}\left(x-x_{j}\right)
\end{gathered}
$$

Substituindo-se estas expressões em (2.7), recupera-se uma importante classe de funções denominadas funções de Shepard, SHEPARD (1968):

$$
\phi_{j}^{0}(\boldsymbol{x})=\frac{W_{j}\left(x-x_{j}\right)}{\sum_{r=1}^{n(\boldsymbol{x})} W_{r}\left(x-x_{r}\right)}
$$

Apesar de poderem representar, de modo exato, apenas constantes, produzindo, por isso, uma aproximação bastante pobre, estas funções são utilizadas com sucesso na família de funções do Método das Nuvens $h p$, como mostrado na próxima seção.

\subsection{Famílias de Funções do Método das Nuvens $h p\left(\Im_{N}^{k, p}\right)$}

Em DUARTE (1996), foi proposta uma nova classe de funções de aproximação, denominada família de Nuvens- $h p$. Tal classe pode ser construída a partir do enriquecimento das aproximações do MMQM multiplicadas por funções, geralmente polinomiais, linearmente independentes. Justifica-se esse procedimento pelo fato destas funções formarem uma Partição da Unidade, PU, cujo o conceito é discutido no apêndice $\mathrm{B}$.

Considera-se que as funções de aproximação (2.7) tenham sido obtidas a partir de uma base $\boldsymbol{P}_{k}=\left\{p_{i}\right\}_{i=1}^{m}$ de $m$ monômios que geram o espaço polinomial de grau máximo $k$, denominado $\mathcal{P}_{k}$. Para se construir uma função $\tilde{u}(\boldsymbol{x})$ que aproxime qualquer polinômio de ordem menor ou igual a $p>k$, definem-se as funções que constituem a família de Nuvens- $h p, \Im_{N}^{k, p}$, como:

$$
\begin{aligned}
\Im_{N}^{k, p} \stackrel{\text { def }}{=} & \left\{\left\{\phi_{j}(\boldsymbol{x})\right\}_{j=1}^{N} \cup\left\{\phi_{j}(\boldsymbol{x}) p_{i}(\boldsymbol{x})\right\}_{j=1}^{N} ;\right. \\
& \left.i=m+1, \cdots, q(p) ; p_{i} \in \mathcal{P}_{p}-\mathcal{P}_{k} ; p \geq k\right\}
\end{aligned}
$$

onde $\mathcal{P}_{p}$ é o espaço polinomial de grau máximo $p$ gerado pela base $\boldsymbol{P}_{p}=\left\{p_{i}\right\}_{i=1}^{q(p)}$ formada por $q(p)$ monômios. Observa-se que, teoricamente, não existe nenhuma restrição 
à forma dos monômios de $\mathcal{P}_{p}-\mathcal{P}_{k}$. É, contudo, aconselhável que sejam normalizados, e anulados nos pontos nodais associados às funções da PU $\left\{\phi_{j}(\boldsymbol{x})\right\}_{j=1}^{N}$. Evita-se, assim, que a aproximação, apesar de construída nas coordenadas globais, seja sensível às dimensões do problema. Em $\mathbb{R}^{2}$, por exemplo, o polinômio de grau $r$ em $x$ e $s$ em $y$, que enriquece a PU associada a um nó $\boldsymbol{x}_{j}$, é representado por:

$$
p_{j i}(\boldsymbol{x})=\left(\frac{x-x_{j}}{h_{j}^{x}}\right)^{r}\left(\frac{y-y_{j}}{h_{j}^{y}}\right)^{s}
$$

sendo $h_{j}^{x}$ e $h_{j}^{y}$ dimensões características da nuvem nas direções $x$ e $y$.

Deve-se, ainda, ressaltar que dependendo das características da função que se quer aproximar, podem ser introduzidos termos não polinomiais na base $\boldsymbol{P}_{p}$.

Substituindo-se as funções definidas em (2.14) na aproximação (2.6) tem-se a expressão final para a função aproximadora do Método das Nuvens $h p$ :

$$
u_{p}(\boldsymbol{x})=\sum_{j=1}^{N} \phi_{j}(\boldsymbol{x})\left\{u_{j}+\sum_{i=m+1}^{q_{j}(p)} p_{j i}(\boldsymbol{x}) b_{j i}\right\}=\boldsymbol{\Phi}^{T} \boldsymbol{U}
$$

onde, assim como para o MGLE, são definidos os vetores de parâmetros nodais e das funções de forma:

$$
\begin{aligned}
& \boldsymbol{U}^{T} \stackrel{\text { def }}{=}\left[\begin{array}{lllllllll}
u_{1} & b_{1 m+1} & \cdots & b_{1 q_{1}} & \cdots & u_{N} & b_{N m+1} & \cdots & b_{N q_{N}}
\end{array}\right] \\
& \boldsymbol{\Phi}^{T} \stackrel{\text { def }}{=}\left[\begin{array}{lllllllll}
\phi_{1} & p_{1 m+1} \phi_{1} & \cdots & p_{1_{1} q(p)} \phi_{1} & \cdots & \phi_{N} & p_{N m+1} \phi_{N} & \cdots & p_{N q_{N}(p)} \phi_{N}
\end{array}\right]
\end{aligned}
$$

sendo $b_{j i}, \quad i=m+1, \cdots, q_{j}(p)$ novos parâmetros associados a $\boldsymbol{x}_{j}$ devido ao enriquecimento que, por sua vez pode variar para cada nó. Por essa razão, a quantidade de funções vinculadas ao nó $\boldsymbol{x}_{j}$ é representada por $q_{j}(p)$, ou seja, é função do nó e da ordem polinomial máxima $p$ que se deseja representar.

Pela forma com que a aproximação (2.16) é construída, pode-se notar o caráter hierárquico do enriquecimento empregado, simplificando bastante a implementação de procedimentos de refinamento $p$-adaptativo. Em DUARTE; ODEN (1996b) demonstra-se que, se o conjunto $\left\{\phi_{j}(\boldsymbol{x})\right\}_{j=1}^{N}$ forma uma PU, a função $u_{p}(\boldsymbol{x})$ pode aproximar exatamente qualquer elemento do espaço de $\mathcal{P}_{p}$. Na seção 2.5, esse assunto é retomado mas sob o enfoque do MEFG. O emprego da notação $u_{p}(\boldsymbol{x})$ no lugar de $\tilde{u}(\boldsymbol{x})$ em (2.16) tem, portanto, o intuito de informar que a aproximação construída re- 
presenta exatamente polinômios de grau máximo $p$. Esta será a representação adotada neste trabalho, inclusive para as aproximações do MGLE. A forma $\tilde{u}(\boldsymbol{x})$ será reservada para os casos gerais, referindo-se também às aproximações capazes de reproduzir funções não-polinomiais, isto é, quando outros tipos de funções estiverem multiplicando a PU. Nesse caso a expressão (2.16) é substituída por:

$$
\tilde{u}(\boldsymbol{x})=\sum_{j=1}^{N} \phi_{j}(\boldsymbol{x})\left\{u_{j}+\sum_{i=1}^{q_{j}} L_{j i}(\boldsymbol{x}) b_{j i}\right\}=\boldsymbol{\Phi}^{T} \boldsymbol{U}
$$

onde $L_{j i}$ corresponde à "i-ésima" função que multiplica a PU do nó $\boldsymbol{x}_{j}$, podendo ser polinomial ou não. Já $q_{j}$ representa o número de funções $L_{j i}$ utilizadas. Para essa representação geral, o vetor das funções de forma é definido como:

$$
\boldsymbol{\Phi}^{T} \stackrel{\text { def }}{=}\left[\begin{array}{lllllllll}
\phi_{1} & L_{11} \phi_{1} & \cdots & L_{1 q_{1}} \phi_{1} & \cdots & \phi_{N} & L_{N 1} \phi_{N} & \cdots & L_{N q_{N}} \phi_{N}
\end{array}\right]
$$

Finalmente, é preciso ressaltar que nas aplicações das aproximações de Nuvens$h p$, DUARTE (1996) e DUARTE; ODEN (1996b), por exemplo, é usual o emprego de funções de Shepard, (2.13), para se construir a PU $\left\{\phi_{j}(\boldsymbol{x})\right\}_{j=1}^{N}$. Como já foi observado na seção anterior, tais funções geram aproximações bastante pobres. Por outro lado, apresentam como vantagens a facilidade de implementação em qualquer dimensão, e o baixo custo computacional, pois não há necessidade de se inverter a matriz $\boldsymbol{A}(\boldsymbol{x})$ em cada posição de cálculo da aproximação $\tilde{u}(\boldsymbol{x})$. As funções de Shepard simplificam, portanto, o processo de construção da PU a ser enriquecida, por sua vez, pela multiplicação de funções adequadas ao tipo de aproximação procurada. Consegue-se assim, um desempenho computacional superior ao das funções de MMQM sem perda da qualidade da aproximação.

\subsection{Formulação de Galerkin para Problemas de Valor de Contorno e Emprego dos Métodos Sem Malha}

Considera-se o seguinte problema de valor de contorno:

$$
\text { Encontrar } u \text { tal que: }\left\{\begin{array}{l}
A u=f \quad \text { em } \Omega \\
B_{k} u=g_{k} \text { sobre } \partial \Omega \quad 0 \leq k \leq m-1
\end{array}\right.
$$


em que $\Omega$ é um sub-conjunto aberto em $\mathbb{R}^{\eta}$, de contorno suave $\partial \Omega$, $A$ é um operador linear diferencial de ordem $2 m,\left\{B_{k}\right\}_{k=0}^{m-1}$ são operadores lineares diferenciais sobre o contorno, enquanto que $f$ e $g_{k}$ são funções prescritas.

Seja, também, o seguinte problema variacional associado:

$$
\text { Encontrar } u \in \mathcal{H} \text { tal que: } \mathcal{B}(u, v)=l(v) \forall v \in \mathcal{H}^{m}
$$

onde $v$ são funções de teste pertencentes ao espaço de Hilbert de ordem $m, \mathcal{H}^{m}, \mathcal{B}$ é uma forma bi-linear de $\mathcal{H}^{m} \times \mathcal{H}^{m} \rightarrow \mathbb{R}$ e $l(\bullet)$ uma forma linear em $\mathcal{H}^{m} \rightarrow \mathbb{R}^{1}$.

Pelo procedimento de Galerkin, ODEN; REDDY (1976), a solução aproximada do PVC deve ser procurada em sub-espaços $\tilde{X}$ de dimensão finita, de tal maneira que se tenha $\tilde{X} \subset \mathcal{H}$. Em NAYROLES; TOUZOT; VILLON (1992), propõe-se, pela primeira vez, o emprego das funções do MMQM como geradoras desses espaços. O método, dessa maneira formulado, foi designado de MED. Em BELYTSCHKO; LU; GU (1994), o MGLE é introduzido como uma formulação aperfeiçoada do MED, especialmente quanto à derivação das funções do MMQM e à imposição das condições de contorno essenciais, ou de Dirichlet. Para entender melhor esse último ponto, deve-se observar que uma das principais características desses métodos, que os diferenciam do MEF, é que a aproximação resultante não é, necessariamente, uma interpolação. Como conseqüência, não satisfaz as condições do delta de Kronecker, ou seja, $\phi_{j}\left(\boldsymbol{x}_{i}\right) \neq \delta_{i j}$ e, por isso, as condições de Dirichlet não podem ser impostas diretamente. O espaço $\tilde{X}$ contém, portanto, funções que não são cinematicamente admissíveis e, por isso, as condições de contorno de Dirichlet são introduzidas na formulação fraca do problema. Em BELYTSCHKO; LU; GU (1994) sugere-se o emprego do Método dos Multiplicadores de Lagrange, ODEN; REDDY (1976). O problema (2.21) pode, dessa maneira, ser reescrito como:

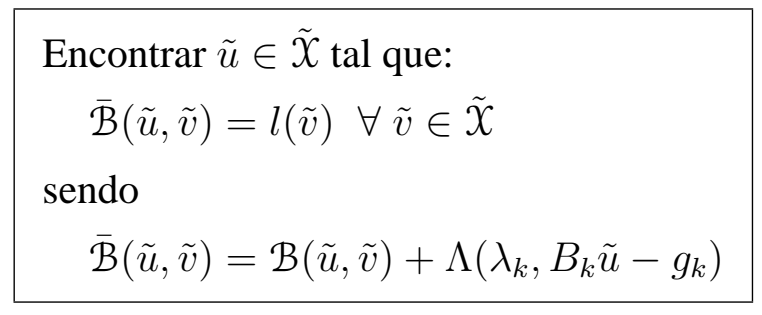

onde $\Lambda(\bullet, \star)$ é o funcional através do qual as condições de contorno essenciais são impostas, $\lambda_{k}$ são multiplicadores de Lagrange e $\tilde{X}$ é o espaço gerado pelas funções de aproximação $\tilde{u}(\boldsymbol{x})$ de (2.10). 
No Método das Nuvens $h p$, o espaço $\tilde{X}$ é obtido utilizando-se a família de Nuvens$h p$, (2.14). Devido ao emprego das funções do MMQM na construção da PU, o mesmo problema, apresentado pelo MGLE com relação à imposição das condições de contorno de Dirichlet é observado. O Método dos Multiplicadores de Lagrange é, portanto, novamente utilizado, resultando em uma expressão equivalente à (2.22) para a aproximação de Galerkin do PVC.

Para que o problema (2.22) possa ser reduzido à solução de um sistema de equações, são introduzidas as aproximações (2.6) e (2.18), conforme a formulação seja do MGLE ou Método das Nuvens $h p$. Como também as funções teste são aproximadas no espaço $\tilde{X}$, suas representações são análogas às aproximações de $u(x)$, ou seja:

$$
v(x) \approx \tilde{v}(x)=\boldsymbol{\Phi}^{T} \boldsymbol{V}
$$

sendo:

- para o MGLE:

$$
\boldsymbol{V}^{T} \stackrel{\text { def }}{=}\left[\begin{array}{llll}
v_{1} & v_{2} & \cdots & v_{N}
\end{array}\right]
$$

- para o Método das Nuvens $h p$ :

$$
\boldsymbol{V}^{T} \stackrel{\text { def }}{=}\left[\begin{array}{lllllllll}
v_{1} & c_{1 m+1} & \cdots & c_{1 q_{1}} & \cdots & v_{N} & c_{N m+1} & \cdots & c_{N q_{N}}
\end{array}\right]
$$

Chega-se, então, ao seguinte sistema de equações:

$$
\overline{\mathcal{B}}\left(\boldsymbol{\Phi}^{T} \boldsymbol{U}, \boldsymbol{\Phi}^{T} \boldsymbol{V}\right)=l\left(\boldsymbol{\Phi}^{T} \boldsymbol{V}\right)
$$

cuja a solução deve ser levada em (2.6) no caso do MGLE ou em (2.18) no caso do Método das Nuvens, para se construir a aproximação.

\subsection{Integração Numérica}

A construção da aproximação via MMQM produz, como mostrado na seção (2.1), uma função que deve ser recalculada em cada posição $\boldsymbol{x}$, impossibilitando a sua descrição analítica. Sendo assim, para as integrações no domínio e no contorno do sistema de 
equações (2.26), deve-se sempre recorrer à uma aproximação numérica. Em BELYTSCHKO; LU; GU (1994), para possibilitar o cálculo das integrais numéricas através da quadratura de Gauss, sugere-se a divisão do domínio em células de integração, onde são distribuídos os pontos de Gauss. Uma explicação detalhada sobre a maneira segundo a qual estas células são distribuídas ao longo do domínio pode ser encontrada em HEGEN (1997).

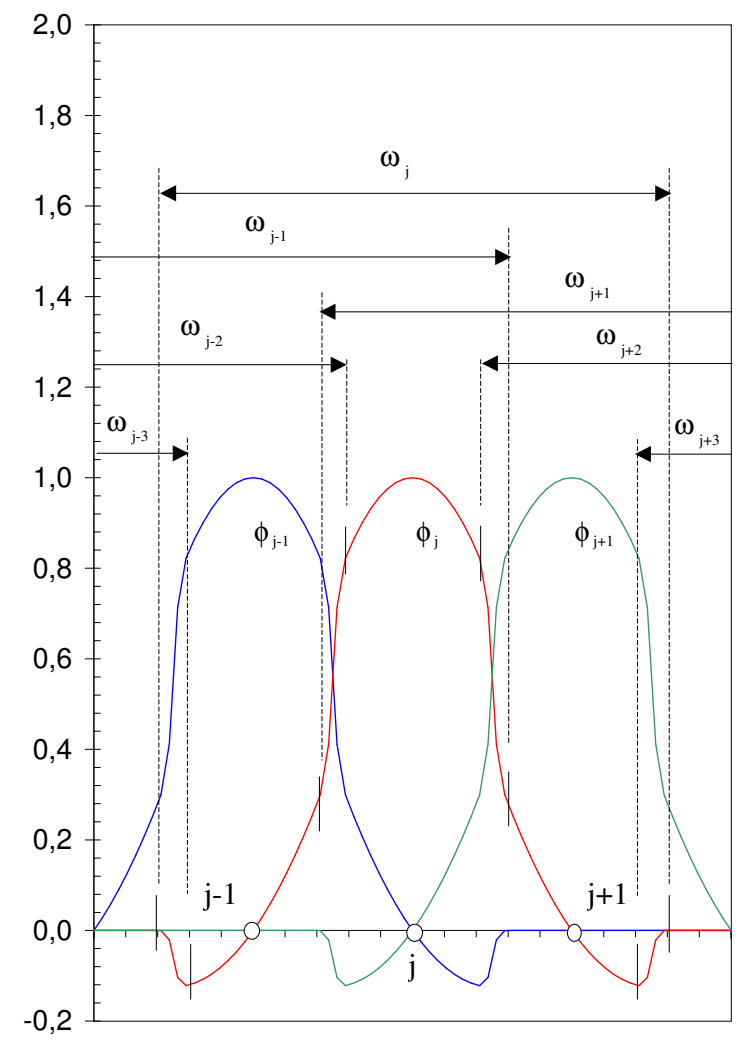

Figura 2.3: Setores da função de forma $\phi_{j}$ - parâmetros empregados: $m=3, R_{j}=1,6 h$, $h \rightarrow$ distância entre os nós - Observar que $\phi_{j}$ verifica a condição do $\delta_{j i}$ para o caso ilustrado em que $m=n$, o que nem sempre é verdadeiro.

Cabe lembrar que a definição de um número do pontos de Gauss para que a integral seja calculada de maneira exata em cada célula não é direta como no MEF. Nesse método, as funções interpoladoras empregadas fazem com que o integrando seja um polinômio e, conseqüentemente, sempre existe um número de pontos de Gauss grande o suficiente para que a integração numérica seja exata. No MGLE e no Método das Nuvens $h p$, independentemente de se ter a base de funções $\boldsymbol{P}$ e/ou a função de ponderação $W_{j}$ polinomiais, a aproximação resultante é geralmente uma função racional 
(quociente de polinômios), devido à inversão da matriz $\boldsymbol{A}(\boldsymbol{x})$ na expressão (2.7). Os integrandos de (2.26) não são portanto polinomiais e, por isso, não há como se ter uma integração exata através da quadratura de Gauss.

Outra fonte de erro para a integração numérica está no desalinhamento entre as células de integração e o suporte das funções de forma que se sobrepõem para construir a função de aproximação. Para melhor explicar esse fato é necessário que se retorne à expressão (2.7). Para cada posição $\boldsymbol{x}$, uma diferente expressão para $\boldsymbol{A}^{-1}(\boldsymbol{x})$ é obtida, influenciada, apenas, pelas nuvens que contêm $\boldsymbol{x}$. As funções de form, portanto, são construídas por setores formados pelas diversas sobreposições de nuvens, conforme exemplifica-se através da Figura 2.3. Em DOLBOW (1998) procura-se fazer com que os contornos das células acompanhem tais setores de sobreposição. A estratégia adotada restringe, todavia, a definição da aproximação pois exige que os suportes sejam retangulares, além de não resolver o problema do caráter não-polinomial das aproximações.

\subsection{Método dos Elementos Finitos Generalizados}

O MEFG, segundo DUARTE; BABUŠKA; ODEN (2000), foi proposto de forma independente por:

- Babuška e colegas sob a denominação de Método dos Elementos Finitos Especiais, BABUŠKA; CALOZ; OSBORN (1994), e como Método Partição da Unidade, MELENK; BABUŠKA (1996); BABUŠKA; MELENK (1997);

- Duarte e Oden a partir de trabalhos como DUARTE; ODEN (1995), DUARTE (1996), DUARTE; ODEN (1996b) e DUARTE; ODEN (1996a) correspondentes à formulação do método das Nuvens e ODEN; DUARTE; ZIENKIEWICZ (1998) como uma formulação híbrida do MEF.

Seu emprego sob a denominação atual de Método dos Elementos Finitos Generalizados surge, contudo, pela primeira vez em MELENK (1995).

A estratégia utilizada no MEFG é bastante simples e consiste em empregar as funções de forma do MEF como PU. Para problemas de elasticidade plana, por exemplo, são empregadas as funções lagrangianas bi-lineares. A dimensão original do espaço de elementos finitos é, em seguida, ampliada através do enriquecimento obtido à maneira do Método das Nuvens $h p$. 


\subsubsection{A Formulação}

De um estudo realizado em DUARTE (1996) sobre o desempenho do MGLE e do Método das Nuvens $h p$, quando comparados ao MEF convencional, argumenta-se que a integração numérica do sistema (2.26) encarece computacionalmente esses métodos, pelas razões já discutidas na seção 2.4. Além desses fatos, pode também ser incluído o problema relacionado à imposição das condições de contorno essenciais pelos multiplicadores de Lagrange, que torna o método instável. Em vista desse quadro, justifica-se o retorno ao tipo de construção das aproximações do MEF procurando-se aproveitar, contudo, avanços obtidos nas formulações sem malha. É o caso do enriquecimento proposto através das famílias de Nuvens- $h p$.

Com o MEFG, os problemas acima são resolvidos. As funções de aproximação típicas do MEF passam a ser interpretadas como PU, cujas nuvens são formados por conjuntos de elementos finitos que concorrem nos pontos nodais $\boldsymbol{x}_{j}$. Para $\mathbb{R}^{1}$, por exemplo, empregando-se as funções de Lagrange lineares mostradas na Figura 2.4, o suporte $\omega_{j}$ da função $\mathcal{N}_{j}(\boldsymbol{x})$ corresponde à união dos elementos $\beta-1$ e $\beta$. Para uma posição no interior do elemento $\beta$, a aproximação inicial pertence ao espaço gerado pelas duas funções de aproximação dos pontos nodais $\boldsymbol{x}_{j}$ e $\boldsymbol{x}_{j+1}$, ou seja:

$$
\tilde{u}(\boldsymbol{x}) \in\left\langle\mathcal{N}_{j}, \mathcal{N}_{j+1}\right\rangle
$$

onde $\langle\bullet, \star, \cdots\rangle$ representa o espaço gerado pelas funções $\bullet, \star, \cdots$.

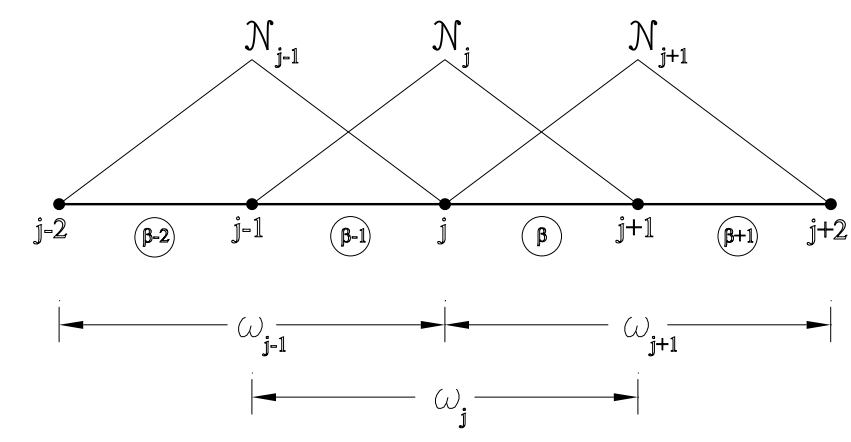

Figura 2.4: Partição da Unidade a partir dos elementos finitos em $\mathbb{R}^{1}$

O enriquecimento, à maneira do Método das Nuvens $h p$, permite que esse espaço seja ampliado, como por exemplo pela multiplicação da função base de cada nó $\boldsymbol{x}_{j}$, pertencente ao elemento $\beta$, por um novo conjunto de funções linearmente independentes $\mathcal{J}_{j}=\left\{1, L_{j 1}, L_{j 2}, \ldots, L_{j q_{j}}\right\}$. Desse modo, para o elemento $\beta$ da Figura 2.4 
obtém-se:

$$
\tilde{u}(\boldsymbol{x}) \in\left\langle\bigcup_{i=1}^{q_{j}} \mathcal{N}_{j} L_{j i}, \bigcup_{i=1}^{q_{j}} \mathcal{N}_{j+1} L_{j+1 i}\right\rangle=\left\langle S_{\beta}\right\rangle
$$

onde $S_{\beta}$ representa as funções obtidas do produto de $\mathcal{J}_{j}$ pela PU no elemento $\beta$.

As funções $L_{j i}$ são, a princípio, quaisquer, podendo formar conjuntos de monômios completos ou não, ou mesmo ser definidas a partir de um conhecimento $a$ priori da solução. São aproximações locais que devem representar bem a solução sobre o suporte a elas associado. Uma vez que a PU permite, como se demonstra em DUARTE; BABUŠKA; ODEN (2000), que as funções introduzidas possam ser reproduzidas a partir de combinações lineares das funções de aproximação definidas em (2.28), tem-se:

$$
\left\{L_{j i}, L_{j+1 i}\right\}_{i=1}^{q_{j}} \in\left\langle S_{\beta}\right\rangle
$$

Utiliza-se, assim, a PU para "gerar" espaços de aproximação local, cujas funções podem ser reproduzidas em cada elemento como parte da aproximação global.

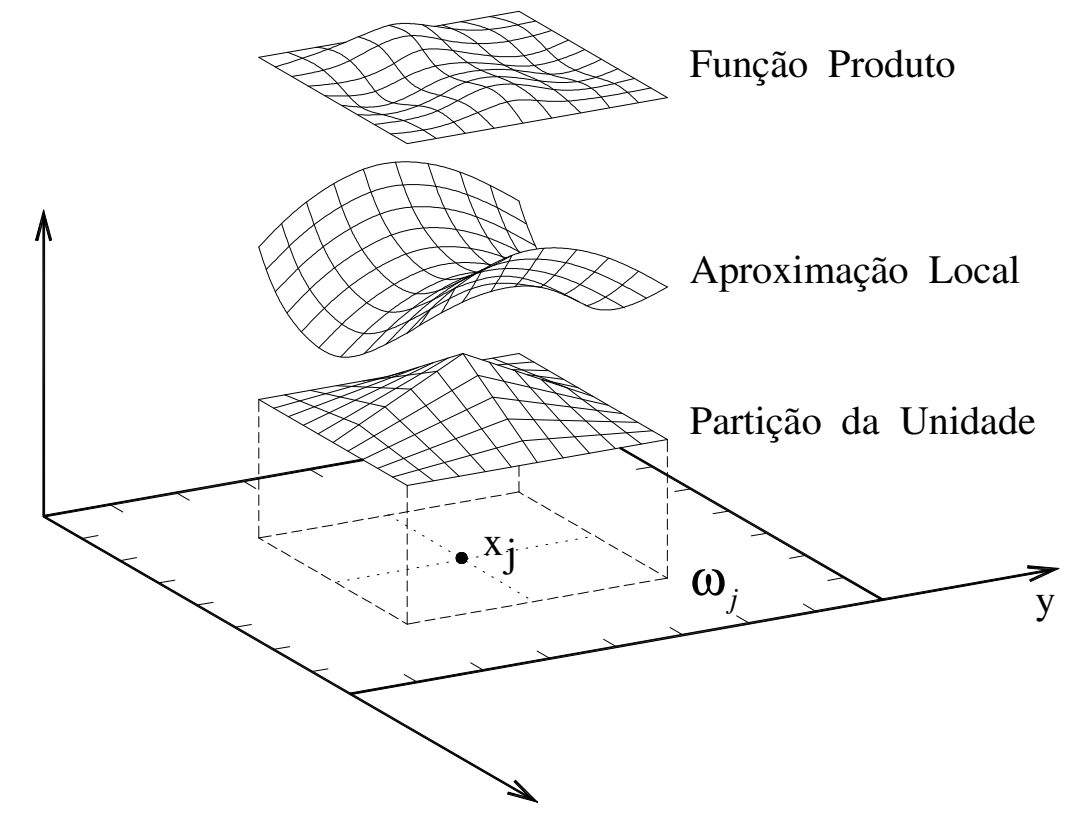

Figura 2.5: Esquema de enriquecimento da Partição da Unidade

A generalização dessa formulação para $\mathbb{R}^{2}$ pode ser melhor entendida a partir da Figura 2.5. Nela, encontram-se representados a PU de ordem 1 (função bi-linear) geradora de uma aproximação $C^{0}$, uma função especial arbitrária e o resultado do produto 
entre essas duas funções. Essa última apresenta as características aproximadoras da função especial, ao mesmo tempo que herda o suporte compacto da PU. Consegue-se, assim, que a aproximação global, obtida em um dado elemento pela combinação das funções produto relativas a cada nó, seja construída sem penalizar a continuidade (no caso do tipo $C^{0}$ ) entre os elementos, que, portanto, se mantêm conformes.

Seguindo-se o mesmo formalismo empregado no Método das Nuvens $h p$ pode-se definir a família de Nuvens- $h p$ para o MEFG:

$$
\Im_{N}^{k=1}=\left\{\left\{\mathcal{N}_{j}(\boldsymbol{x})\right\}_{j=1}^{N} \cup\left\{\mathcal{N}_{j}(\boldsymbol{x}) L_{j i}(\boldsymbol{x})\right\}_{j=1}^{N} \mid i \in \mathcal{J}_{j}\right\}
$$

utilizada para construir a seguinte aproximação:

$$
\tilde{u}(\boldsymbol{x})=\sum_{j=1}^{N} \mathcal{N}_{j}(\boldsymbol{x})\left\{u_{j}+\sum_{i=1}^{q_{j}} L_{j i}(\boldsymbol{x}) b_{j i}\right\}=\boldsymbol{\Phi}^{T} \boldsymbol{U}
$$

em que:

$$
\begin{aligned}
& \boldsymbol{U}^{T} \stackrel{\text { def }}{=}\left[\begin{array}{lllllllll}
u_{1} & b_{11} & \cdots & b_{1 q_{j}} & \cdots & u_{N} & b_{N 1} & \cdots & b_{N q_{j}}
\end{array}\right] \\
& \boldsymbol{\Phi}^{T} \stackrel{\text { def }}{=}\left[\begin{array}{llllllllll}
\phi_{1} & L_{11} \mathcal{N}_{1} & \cdots & L_{1 q_{j}} \mathcal{N}_{1} & \cdots & \phi_{N} & L_{N 1} \mathcal{N}_{N} & \cdots & L_{N q_{j}} \mathcal{N}_{N}
\end{array}\right]
\end{aligned}
$$

Empregando-se essa função em (2.22) para definir o espaço $\tilde{X}$, chega-se à seguinte aproximação de Galerkin, na abordagem do MEFG, para o PVC (2.20):

$$
\begin{aligned}
& \text { Encontrar } \tilde{u} \in \tilde{X} \text { tal que: } \\
& \mathcal{B}(\tilde{u}, \tilde{v})=l(\tilde{v}) \quad \forall \tilde{v} \in \tilde{X}
\end{aligned}
$$

que resulta no seguinte sistema de equações:

$$
\mathcal{B}\left(\boldsymbol{\Phi}^{T} \boldsymbol{U}, \boldsymbol{\Phi}^{T} \boldsymbol{V}\right)=l\left(\boldsymbol{\Phi}^{T} \boldsymbol{V}\right)
$$

onde:

$$
\boldsymbol{V}^{T} \stackrel{\text { def }}{=}\left[\begin{array}{lllllllll}
v_{1} & b_{11} & \cdots & c_{1 q_{j}} & \cdots & v_{N} & c_{N 1} & \cdots & c_{N q_{j}}
\end{array}\right]
$$

A exemplo do que foi comentado para o Método das Nuvens $h p$, caso as funções $L_{i j}$ sejam todas polinomiais definindo a família $\Im_{N}^{k=1, p} \operatorname{como~em~(2.14),~a~aproximação~}$ $\tilde{u}$ será representada de modo particular como: 


$$
u_{p}(\boldsymbol{x})=\sum_{j=1}^{N} \mathcal{N}_{j}(\boldsymbol{x})\left\{u_{j}+\sum_{i=1}^{q_{j}(p)} p_{j i}(\boldsymbol{x}) b_{j i}\right\}=\boldsymbol{\Phi}^{T} \boldsymbol{U}
$$

sendo:

$$
\boldsymbol{\Phi}^{T} \stackrel{\text { def }}{=}\left[\begin{array}{lllllllll}
\phi_{1} & p_{11} \mathcal{N}_{1} & \cdots & p_{1 q_{j}(p)} \mathcal{N}_{1} & \cdots & \phi_{N} & p_{N 1} \mathcal{N}_{N} & \cdots & p_{N q_{j}(p)} \mathcal{N}_{N}
\end{array}\right]
$$

Pela maneira com que se realiza o enriquecimento, chega-se a uma aproximação sem "costura", DUARTE; BABUŠKA; ODEN (2000), ou seja, sem a necessidade de se estabelecer, como ocorre no MEF hierárquico, DEMKOWICZ (1989), condições de restrição que garantam a continuidade dos campos aproximadores entre cada elemento. O emprego das funções do MEF para a PU evita problemas encontrados com as funções do MMQM, como aquele relativo à integração numérica, pois os integrandos de (2.34) tornam-se polinomiais; a menos, é claro, nos casos de elementos distorcidos ou quando o enriquecimento não for polinomial. Outra vantagem consiste na possibilidade de se impor diretamente as condições de contorno essenciais, pois se recupera o caráter interpolador da aproximação.

Um problema proveniente dessa estratégia de enriquecimento é a possibilidade de se ter a família $\Im_{N}^{k=1, p}$ formando um conjunto de funções linearmente dependentes, DUARTE; BABUŠKA; ODEN (2000). Isso ocorre, basicamente, quando se enriquece com monômios uma PU polinomial. O mesmo não acontece para o Método das $\mathrm{Nu}$ vens $h p$, em que a PU é construída por funções não polinomiais, ou polinomiais por setores. A consequiência imediata desse fato é que o sistema de equações (2.34) passa a apresentar uma matriz de rigidez semi-definida positiva, mesmo após a eliminação dos movimentos de corpo rígido. Tal problema pode ser contornado empregando-se as estratégias numéricas sugeridas em STROUBOULIS; BABUŠKA; COPPS (2000), entre elas um método iterativo aqui denominado de procedimento de Babuška. Mais detalhes sobre esse assunto podem ser encontrados no apêndice $\mathrm{C}$.

A obtenção de uma matriz de rigidez singular é, entretanto, um preço razoável a ser pago se comparado à potencialidade do MEFG. De fato, além da facilidade com que se realiza o enriquecimento, novas funções podem ser introduzidas na aproximação conforme o tipo de aplicação desejada. Abre-se assim, a possibilidade de se empregar o MEFG, de maneira eficiente, em problemas em que o campo de tensões apresente singularidades, como no caso de meios com presença de descontinuidades. 


\section{Capítulo 3}

\section{Experimentos Numéricos em Análise Linear}

Neste capítulo, são reunidos exemplos de problemas em análise linear de estruturas, resolvidos através do MGLE, do Método das Nuvens e do MEFG. Uma das razões para a presença desses problemas é, naturalmente, didática. Pretende-se compartilhar, com interessados neste trabalho, as experimentações numéricas que possibilitaram uma melhor compreensão dos novos conceitos introduzidos com as formulações sem malha e de finitos generalizados. Para isso, resultados da literatura, obtidos com o MEF, são recuperados e contrapostos às análises aqui apresentadas. Procura-se, também, evidenciar alguns aspectos da implementação numérica, nem sempre muito claros nos artigos revisados para este trabalho.

Sendo assim, inicia-se com o exemplo uni-dimensional no qual as implementações para o MGLE e o Método das Nuvens $h p$ são descritas e os resultados numéricos apresentados para diferentes situações de análise. O MEFG, por sua vez, é empregado na solução de problemas da elasticidade linear estática em domínio bidimensional. São exemplos selecionados especialmente para estabelecer um confronto entre o MEFG e o MEF, no que se refere à convergência da solução e influência do tipo de malha empregado. Procura-se mostrar que a estratégia de enriquecimento do MEFG confere ao método uma certa independência com relação à discretização. Tal característica simplifica bastante sua aplicação em um procedimento $p$ adaptativo de correção da solução, discutido no capítulo 6. O último exemplo, em especial, ilustra a versatilidade que o método adquire com a possibilidade de se enriquecer a aproximação com funções não-polinomiais. O objetivo final é justificar sua escolha para o estudo da propagação de descontinuidades, especialmente no regime de transição dano-fratura. 


\subsection{Métodos Sem Malha Aplicados no Estudo da Asso- ciação Contínua de Painéis Parede e Pórtico}

Na Figura 3.1, encontra-se representada a estrutura de um edifício alto mediante a associação plana de elementos estruturais denominados painéis parede e pórtico, dispostos em série e ligados por meio de barras bi-articuladas de rigidez axial infinita. Tais barras simulam aproximadamente, de acordo com a Técnica do Meio Contínuo, STAMATO (1972), o efeito das lajes, supostas continuamente distribuídas ao longo da altura e de rigidez infinita no seu plano. O carregamento externo é constituído, conforme ilustra a Figura 3.1(a), por uma força concentrada $F$ aplicada no topo e uma força distribuída $q$ ao longo da altura da associação. Tal carregamento será absorvido pelos painéis de parede e pórtico, nas parcelas $q_{w}$ e $F_{w}$, Figura 3.1(b) e $q_{f}$ e $F_{f}$, Figura $3.1(\mathrm{c})$, respectivamente.

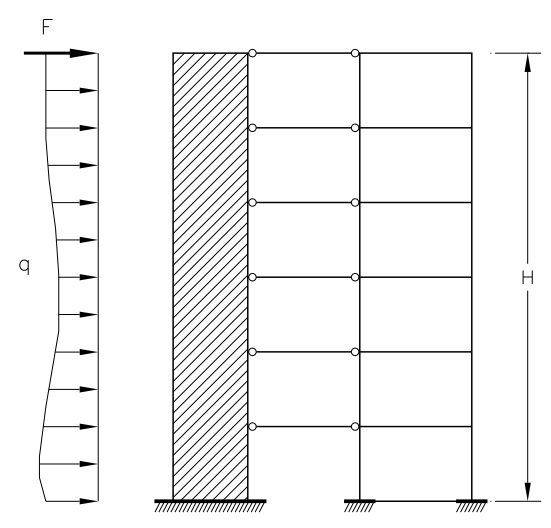

(a) Geometria e carregamento
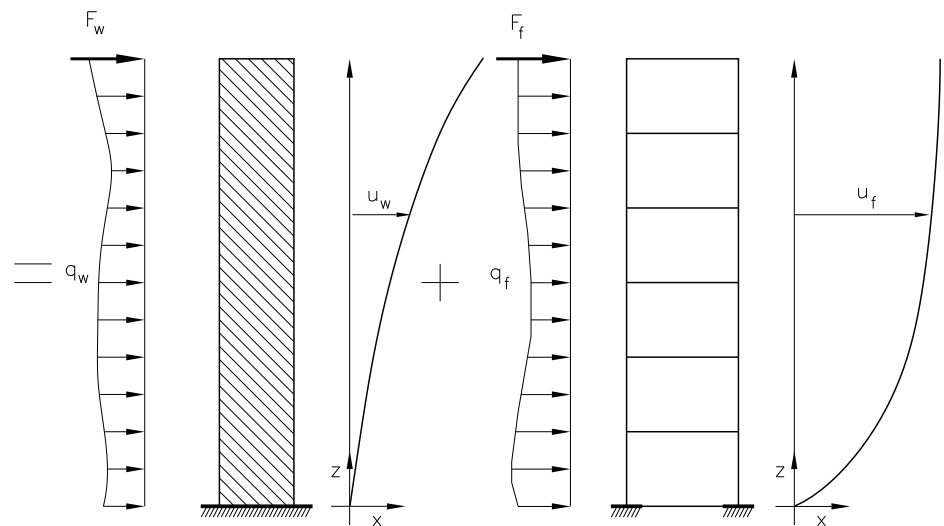

(b) Painel parede

(c) Painel pórtico

Figura 3.1: Associação parede-pórtico

Considerando-se as hipóteses de que a parede se deforma por flexão e o pórtico por cortante, tomando-se $F=0$ e desprezando-se a contribuição dos deslocamentos axiais, formula-se o funcional de energia potencial total, SAVASSI (1975), para o problema em questão como:

$$
\Pi=\overbrace{\int_{0}^{H}\left[\frac{1}{2} j_{w}\left(u^{\prime \prime}\right)^{2}+\frac{1}{2} s_{f}\left(u^{\prime}\right)^{2}\right] d z}^{\mathcal{U}}-\overbrace{\int_{0}^{H} q u d z}^{\mathcal{W}_{\text {ext }}}
$$

onde $j_{w}$ e $s_{f}$ correspondem às rigidezes à flexão e ao cisalhamento respectivamente, 
$u(z)$ é o deslocamento horizontal, $\mathcal{U}$ a energia de deformação e $\mathcal{W}_{\text {ext }}$ o trabalho das forças externas.

As condições de contorno essenciais do conjunto correpondem a se ter deslocamento e rotação nulos para $z=0$, ou seja, $u(0)=0$ e $u^{\prime}(0)=0$.

Substituindo-se $u(z)$ pela aproximação $\tilde{u}(z)$, construída sobre um conjunto de $N$ nós, como em (2.6) para o MGLE ou como em (2.16) para o Método das Nuvens $h p$, obtém-se a seguinte forma discreta para o funcional (3.1):

$$
\Pi=\int_{0}^{H}\left[\frac{1}{2} j_{w}\left(\boldsymbol{\Phi}^{\prime \prime T} \boldsymbol{U}\right)^{2}+\frac{1}{2} s_{f}\left(\boldsymbol{\Phi}^{\prime T} \boldsymbol{U}\right)^{2}-q\left(\boldsymbol{\Phi}^{T} \boldsymbol{U}\right)\right] d z
$$

Deve-se lembrar, contudo, que a função $\tilde{u}(z)$, obtida pelo MMQM, é apenas uma aproximação. Desse modo, como discutido na seção 2.3, não há como impor diretamente as condições de contorno essenciais. Recorre-se assim, ao Método dos Multiplicadores de Lagrange que satisfaz as condições de contorno dentro da formulação fraca do problema. Para isso, define-se um novo funcional $\bar{\Pi}$ obtido como:

$$
\bar{\Pi}=\Pi+\int_{\Gamma_{u}} \lambda^{u}(z)(u(z)-0) d z+\int_{\Gamma_{u}} \lambda^{\theta}(z)\left(u^{\prime}(z)-0\right) d z
$$

onde $\lambda^{u}(z)$ e $\lambda^{\theta}(z)$ são multiplicadores de Lagrange vinculados ao deslocamento e à sua derivada, respectivamente. Já $\Gamma_{u}$ (na verdade, $z=0$ ) corresponde à região do contorno $\Gamma$ onde se encontram prescritas as condições essenciais. Para a obtenção da representação discreta para $\bar{\Pi}$, os multiplicadores de Lagrange são descritos através de valores $\lambda_{1}^{u}$ e $\lambda_{1}^{\theta}$ definidos no ponto nodal 1 localizado em $z=0$ :

$$
\begin{aligned}
& \lambda^{u}(0)=\lambda_{1}^{u} \\
& \lambda^{\theta}(0)=\lambda_{1}^{\theta}
\end{aligned}
$$

A forma final aproximada para o funcional $\bar{\Pi}$ pode ser, então, representada como:

$$
\bar{\Pi}=\Pi+\left.\lambda_{1}^{u}\left(\boldsymbol{\Phi}^{T} \boldsymbol{U}-0\right)\right|_{z=0}+\left.\lambda_{1}^{\theta}\left(\boldsymbol{\Phi}^{\prime T} \boldsymbol{U}-0\right)\right|_{z=0}
$$

Aplicando-se o princípio da estacionaridade, chega-se ao seguinte PVC, equivalente à formulação do Método de Galerkin definida em (2.22): 


$$
\begin{aligned}
\delta \bar{\Pi}=0 \Rightarrow & \delta \boldsymbol{U}^{T}\left\{\int_{0}^{H}\left[j_{w}\left(\boldsymbol{\Phi}^{\prime \prime} \boldsymbol{\Phi}^{\prime \prime T}\right)+s_{f}\left(\boldsymbol{\Phi}^{\prime} \boldsymbol{\Phi}^{\prime T}\right)\right] d z \boldsymbol{U}+\right. \\
& \left.\int_{0}^{H} q \boldsymbol{\Phi} d z+\left.\boldsymbol{\Phi}\right|_{z=0} \lambda_{1}^{u}+\left.\boldsymbol{\Phi}^{\prime}\right|_{z=0} \lambda_{1}^{\theta}\right\}+ \\
& \left\{\left.\delta \lambda_{1}^{u} \boldsymbol{\Phi}^{T}\right|_{z=0}+\left.\delta \lambda_{1}^{\theta} \boldsymbol{\Phi}^{\prime T}\right|_{z=0}\right\} \boldsymbol{U}=0
\end{aligned}
$$

Como a expressão (3.6) deve ser válida para quaisquer valores de $\delta \boldsymbol{U}, \delta \boldsymbol{\lambda}^{u}$ e $\delta \boldsymbol{\lambda}^{\theta}$, deve-se ter separadamente:

$$
\begin{gathered}
{\left[\int_{0}^{H} j_{w}\left(\boldsymbol{\Phi}^{\prime \prime} \boldsymbol{\Phi}^{\prime \prime T}\right) d z+\int_{0}^{H} s_{f}\left(\boldsymbol{\Phi}^{\prime} \boldsymbol{\Phi}^{\prime T}\right) d z\right] \boldsymbol{U}-\int_{0}^{H} \boldsymbol{\Phi} q d z+} \\
\left\{\left.\boldsymbol{\Phi}\right|_{z=0}\right\} \lambda_{1}^{u}+\left\{\left.\boldsymbol{\Phi}^{\prime}\right|_{z=0}\right\} \lambda_{1}^{\theta}=\mathbf{0} \\
\left\{\left.\boldsymbol{\Phi}^{T}\right|_{z=0}\right\} \boldsymbol{U}=\mathbf{0} \\
\left\{\left.\boldsymbol{\Phi}^{\prime T}\right|_{z=0}\right\} \boldsymbol{U}=\mathbf{0}
\end{gathered}
$$

A equação (3.6) pode, portanto, ser escrita em formulação compacta da seguinte maneira:

$$
\overbrace{\left[\begin{array}{ccc}
\boldsymbol{K} & \boldsymbol{G}^{u} & \boldsymbol{G}^{\theta} \\
\left(\boldsymbol{G}^{u}\right)^{T} & \boldsymbol{0} & \boldsymbol{0} \\
\left(\boldsymbol{G}^{\theta}\right)^{T} & \mathbf{0} & \mathbf{0}
\end{array}\right]}^{\widehat{\boldsymbol{K}} \operatorname{de}\left[\left(N^{*}+2\right) \times\left(N^{*}+2\right)\right]} \overbrace{\left\{\begin{array}{c}
\boldsymbol{U} \\
\lambda_{1}^{u} \\
\lambda_{1}^{\theta}
\end{array}\right\}}^{\widehat{\boldsymbol{U}} \operatorname{de}\left[N^{*}+2\right]}=\overbrace{\left\{\begin{array}{c}
\boldsymbol{f} \\
\mathbf{0} \\
\mathbf{0}
\end{array}\right\}}^{\widehat{\boldsymbol{F}} \mathrm{de}\left[N^{*}+2\right]}
$$

onde:

- $K_{i j}=\int_{0}^{H}\left[j_{w}\left(\boldsymbol{\Phi}^{\prime \prime} \boldsymbol{\Phi}^{\prime \prime T}\right)_{i j}\right] d z+\int_{0}^{H}\left[s_{f}\left(\boldsymbol{\Phi}^{\prime} \boldsymbol{\Phi}^{\prime T}\right)_{i j}\right] d z$

- $f_{i}=\int_{0}^{H}\left[q \Phi_{i}(z)\right] d z$

- $G_{i}^{u}=\Phi_{i}(0) ; G_{i}^{\theta}=\Phi_{i}^{\prime}(0) ; \quad$ para $i, j=2, \cdots, N^{*}$

- $N^{*}$ é o número de funções de forma empregado (número de graus de liberdade). Caso a análise esteja sendo feita pelo MGLE, $N^{*}=N$. Por outro lado, sendo 
empregado o Método das Nuvens $h p, N^{*}$ depende do número de funções enriquecedoras, ou seja, $N^{*}=\sum_{j=1}^{N} q_{j}$, sendo $q_{j}$ definido na seção 2.2.

Cabe ainda observar que os parâmetros $u_{i}$ obtidos com a solução de $\widehat{\boldsymbol{U}}$ não definem, diretamente, os deslocamentos nodais, pois a aproximação não corresponde, necessariamente, a uma interpolação. Caso se queira determinar os deslocamentos nodais, deve-se realizar um pós-processamento empregando-se, novamente, as expressões (2.6) para o MGLE e em (2.16) para o Método das Nuvens $h p$.

Com relação à parcela $K_{i j}$ da matriz de rigidez, é interessante notar que sua largura de banda depende do tamanho dos domínios de influência $\omega_{i}$ de cada ponto nodal $z_{i}$. Quanto maior o raio de $\omega_{i}$ maior o número de pontos presentes em cada nuvem e, conseqüentemente, maior a largura de banda da matriz de rigidez.

\subsubsection{Análise numérica}

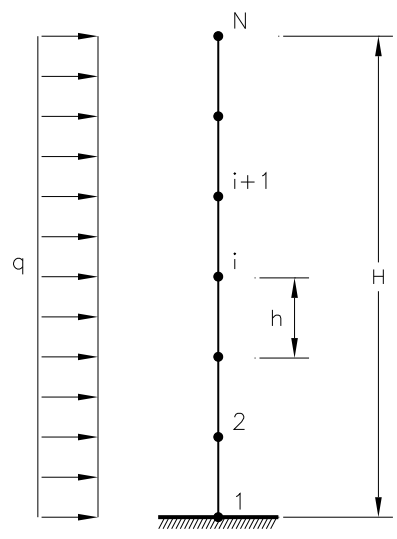

Figura 3.2: Discretização da associação parede e pórtico

Na Figura 3.2, encontram-se representados o carregamento e a distribuição nodal adotados para o problema de associação parede-pórtico. A solução exata do problema, obtida em STAMATO (1972), é:

$$
u(z)=\frac{1}{k_{r}^{4} j_{w}}\left[C_{1}+C_{2} k_{r} z+C_{3} e^{k_{r} z}+C_{4} e^{-k_{r} z}-q \frac{k_{r}^{2} z^{2}}{2}\right]
$$

onde: $k_{r}=\sqrt{s_{f} / j_{w}} ; \quad K_{r}=k_{r} H ; \quad C=2 \cosh K_{r} ; \quad S=2 \sinh K_{r}$;

$$
C_{2}=K_{r} q ; \quad C_{1}=-\frac{1}{C}\left(2 q+C_{2} S\right) ; \quad C_{3}=\frac{1}{C}\left(q-C_{2} e^{-K_{r}}\right) ; \quad C_{4}=\frac{1}{C}\left(q+C_{2} e^{K_{r}}\right)
$$


Para a realização da análise numérica através do MGLE e do Método das Nuvens, adotam-se $s_{f}=1, H=1$ e $q=1$, todos valores adimensionais, considerando-se ainda:

ordem da base polinomial : como em (3.7) aparecem derivadas de segunda ordem das funções de forma, a aproximação deve representar de modo exato, no mínimo, polinômios completos do segundo grau para que a convergência monotônica da solução seja garantida (completidade). Desse modo, adotou-se para o MGLE bases de monômios $\boldsymbol{P}_{k=2}=\left\{\begin{array}{lll}1 & z & z^{2}\end{array}\right\}$ e para o Método das Nuvens $h p$ famílias de funções $\Im_{N}^{k=0, p=2}$, em que a base empregada para a construção das funções de forma $\phi_{j}$ foi $\boldsymbol{P}_{k=0}=\{1\}$ (funções de Shepard) enriquecidas pelas bases $\boldsymbol{P}_{p=2}=\left\{\begin{array}{lll}1 & z & z^{2}\end{array}\right\}$;

aproximação do domínio : uma vez que a solução a ser aproximada é suave, os nós foram distribuídos uniformemente ao longo de $z$, Figura 3.2. Foi utilizada uma sequiência de malhas com $N=6,11,21$ e 41 nós, representando os diferentes níveis de refinamento $h$ da solução;

função de ponderação : para a construção das funções de aproximação do MMQM, foi empregada uma função do tipo spline cúbica, já utilizada em DOLBOW; BELYTSCHKO (1998):

$$
W_{j}\left(z-z_{j}\right) \equiv f(r)=\left\{\begin{array}{cc}
\frac{2}{3}-4 r^{2}+4 r^{3} & \text { para } r \leq \frac{1}{2} \\
\frac{4}{3}-4 r+4 r^{2}-\frac{4}{3} r^{3} & \frac{1}{2}<r \leq 1
\end{array}\right.
$$

onde $r=\frac{\left|z-z_{j}\right|}{R_{j}}$. Estas funções são $C_{0}^{2}\left(\omega_{j}\right)$, o que faz com que as funções de forma também o sejam, satisfazendo, portanto, as exigências relativas à regularidade da aproximação para um problema que é de classe $C^{1}$. Para o Método das Nuvens adotou-se $R_{j}=h, \forall j$, garantindo-se que nenhum ponto de integração pertença a apenas uma nuvem. Alías, essa condição é necessária, segundo MENDONÇA; BARCELLOS; DUARTE (2000), para que a taxa de convergência do método não seja comprometida. Já para o MGLE, que exige um mínimo de 3 nuvens cobrindo cada ponto de integração para contrabalançar o número de monômios da base $\boldsymbol{P}_{k}$, deve-se ter $R_{j} \geq 2 h$. Após alguns testes com diferentes valores para ao raio, adotou-se $R_{j}=10 h$; 
integração numérica : a integração numérica dos termos de $\widehat{\boldsymbol{K}}$ foi realizada através da quadratura de Gauss-Legendre. Como células de integração, foram definidas as regiões localizados entre cada nó, nas quais os pontos da quadratura são distribuídos. Como a aproximação é obtida pela sobreposição de diversos polinômios, um grande número de pontos de Gauss é necessário. A partir de 20 pontos $(N G=20)$ observou-se que o erro na integração numérica não compromete mais a solução.

análise quanto à convergência : para a análise da convergência utilizou-se a norma $L_{2}$ do erro da aproximação, $\|u-\tilde{u}\|_{L_{2}}=\left\{\int_{0}^{H}[u-\tilde{u}]^{2}\right\}^{1 / 2} d z$. A função $u$ é calculada pela expressão (3.9). Já $\tilde{u}$ é definida pelas aproximações (2.6) ou (2.16) conforme a solução tenha sido obtida pelo MGLE ou pelo Método das Nuvens $h p$ respectivamente. A taxa de convergência para o refinamento $h^{1}$ corresponde à inclinação do trecho assintótico da curva que relaciona $\log \left(\|u-\tilde{u}\|_{L_{2}}\right)$ com $\log (h)$.

Os gráficos das Figuras 3.3(a) e 3.3(b) mostram as taxas de convergência $h$ para o MGLE e o Método das Nuvens quando se varia a relação de rigidez medida por $K_{r}$. Segundo OLIVEIRA (1982), caso $K_{r}<5$, o comportamento da estrutura está mais próximo ao de uma viga engastada, cuja solução exata é um polinômio do quarto grau. Já para $K_{r} \geq 5$ a rigidez ao cisalhamento do pórtico torna-se preponderante e a solução perde o caráter polinomial. Por essa razão, um erro maior é introduzido na aproximação numérica, como se observa em ambos os gráficos para os maiores valores de $K_{r}$. As taxas de convergência, entretanto, não são perturbadas, mantendose equivalentes às encontradas para o MEF, ou seja, em torno de $3^{2}$.

Na tabela 3.1 são apresentados os tempos do processamento para as análises com $K_{r}=20$ realizadas pelo MGLE e o Método das Nuvens. Como neste trabalho não houve a preocupação em se utilizar um algoritmo otimizado, estes tempos fornecem apenas informações quanto à ordem de grandeza da duração de tais análises. Além disso, podem também ser utilizados com o intuito de se comparar as análises por esses dois métodos sem malha. Os tempos obtidos permitem concluir por um maior esforço

\footnotetext{
${ }^{1}$ A convergência $h$ refere-se à introdução de novos pontos nodais, de forma análoga ao MEF, em que novos elementos são acrescentados no refinamento do tipo $h$.

${ }^{2}$ De ODEN; REDDY (1976), para um PVC homogêneo de ordem $2 m$, aproximado por Galerkin e sob certas condições, deve-se esperar que $\|u-\tilde{u}\|_{H^{\alpha}(\Omega)} \leq C h^{v}\|f\|_{H^{\lambda}(\Omega)}$, onde $C$ é uma constante independente de $h, \lambda$ é função do grau de regularidade de $f, p$ é a ordem polinomial máxima capaz de ser aproximada por $\tilde{u}$ e $v=\min (p+1-\alpha, 2(p+1-m), 2 m+\lambda-\alpha)$. Para a análise, como o problema é regular $v=p+1-\alpha=3$ para norma $L_{2}(\alpha=0)$.
} 
computational exigido com o MGLE. Esse fato já era esperado pelas necessidades de se inverter a matriz $\boldsymbol{A},(2.4)$, e de envolver um maior número de pontos para a construção da aproximação no MGLE. Em análises bi e tri-dimensionais espera-se que tal observação seja potencializada em favor do Método das Nuvens.

\begin{tabular}{|c|c|c|}
\hline NN & MGLE & Nuvens \\
\hline \hline 6 & $0,513 \mathrm{~s}$ & $0,313 \mathrm{~s}$ \\
\hline 11 & $0,750 \mathrm{~s}$ & $0,607 \mathrm{~s}$ \\
\hline 21 & $1,207 \mathrm{~s}$ & $0,823 \mathrm{~s}$ \\
\hline 31 & $2,763 \mathrm{~s}$ & $1,923 \mathrm{~s}$ \\
\hline
\end{tabular}

Tabela 3.1: Tempo de processamento para as várias distribuições nodais (NN) com os métodos sem malha $-K_{r}=20, p=2$

Já nas Tabelas (3.2), (3.3), (3.4), (3.5), os resultados obtidos para $K_{r}=20$, com diferentes distribuições nodais, são confrontados com as análise feitas através do MEF em OLIVEIRA (1982), no qual adotou-se uma aproximação do terceiro grau. Para isso, foram utilizadas aproximações cúbicas tanto no MGLE $\left(\boldsymbol{P}_{k=2}\right)$ quanto para o Método das Nuvens $\left(\Im_{N}^{k=0, p=3}\right)$. Optou-se por comparar as soluções dos esforços cortante no pórtico e momento fletor na parede. Tais esforços estão relacionados às derivadas de ordens primeira e segunda dos deslocamentos respectivamente e, por isso, devem apresentar um erro de aproximação maior do que aquele que seria observado para $\tilde{u}(z)$.

O método que mais se afastou dos resultados analíticos foi o MGLE, particularmente para 6 pontos nodais. Com a introdução de novos pontos, essa diferença se reduz rapidamente comprovando a boa taxa de convergência observada no gráfico da Figura 3.3(a). No Método das Nuvens $h p$, os resultados mostram-se, em geral, mais próximos da solução analítica, mesmo para os 6 pontos nodais.

\begin{tabular}{|c|cc|cc|cc|c|}
\hline \multirow{2}{*}{$\begin{array}{c}\text { Posição } \\
\text { z }\end{array}$} & \multicolumn{2}{|c|}{ 6 nós } & \multicolumn{2}{c|}{ 11 nós } & \multicolumn{2}{c|}{ 21 nós } & Solução \\
\cline { 2 - 7 } analítica
\end{tabular}

Tabela 3.2: Esforço cortante no pórtico - $K_{r}=20$, base $\boldsymbol{P}_{k=3}$ 


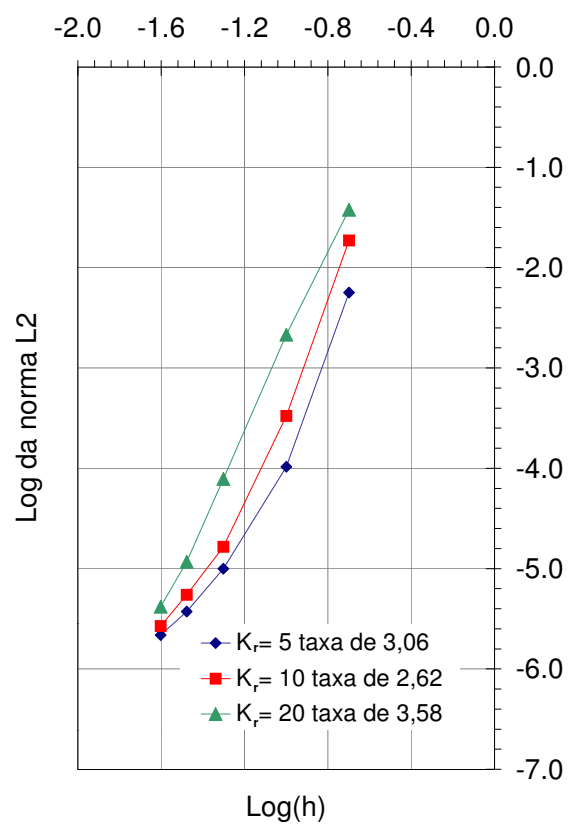

(a) MGLE- $\boldsymbol{P}_{k=2}$

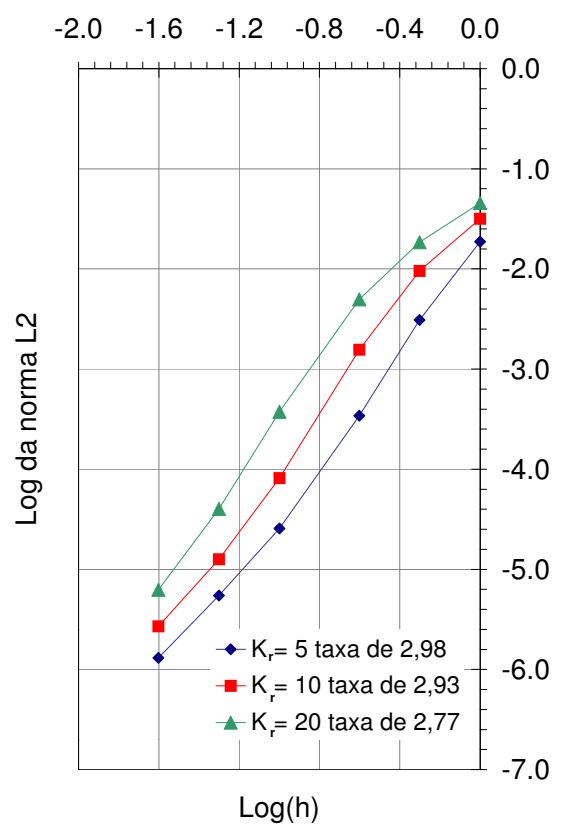

(b) Método das Nuvens $h p-\Im_{N}^{k=0, p=2}$

Figura 3.3: Influência da rigidez relativa $K_{r}$

\begin{tabular}{|c|cc|cc|cc|c|}
\hline \multirow{2}{*}{$\begin{array}{c}\text { Posição } \\
\text { z }\end{array}$} & \multicolumn{2}{|c|}{ 6 nós } & \multicolumn{2}{c|}{ 11 nós } & \multicolumn{2}{c|}{ 21 nós } & Solução \\
\cline { 2 - 7 } & MGLE & MEF & MGLE & MEF & MGLE & MEF & analítica \\
\hline 0,0 & 0,0194 & 0,0295 & 0,0309 & 0,0393 & 0,0465 & 0,0446 & 0,0475 \\
0,2 & 0,0029 & $-0,0010$ & $-0,0036$ & $-0,0016$ & $-0,0016$ & $-0,0016$ & $-0,0016$ \\
0,4 & $-0,0055$ & $-0,0024$ & $-0,0030$ & $-0,0024$ & $-0,0025$ & $-0,0024$ & $-0,0025$ \\
0,6 & $-0,0015$ & $-0,0025$ & $-0,0022$ & $-0,0024$ & $-0,0025$ & $-0,0024$ & $-0,0025$ \\
0,8 & $-0,0020$ & $-0,0028$ & $-0,0042$ & $-0,0024$ & $-0,0026$ & $-0,0024$ & $-0,0025$ \\
1,0 & $-0,0037$ & $-0,0009$ & 0,0030 & $-0,0004$ & 0,0002 & $-0,0001$ & 0,0000 \\
\hline
\end{tabular}

Tabela 3.3: Esforço momento fletor na parede- $K_{r}=20$, base $\boldsymbol{P}_{k=2}$

\begin{tabular}{|c|cc|cc|cc|c|}
\hline \multirow{2}{*}{$\begin{array}{c}\text { Posição } \\
\text { z }\end{array}$} & \multicolumn{2}{|c|}{ 6 nós } & \multicolumn{2}{c|}{ 11 nós } & \multicolumn{2}{c|}{ 21 nós } & Solução \\
\cline { 2 - 7 } & Nuvens & MEF & Nuvens & MEF & Nuvens & MEF & analítica \\
\hline 0,0 & 0,0000 & 0,0000 & 0,0000 & 0,0000 & 0,0000 & 0,0000 & 0,0000 \\
0,2 & 0,7705 & 0,7549 & 0,7808 & 0,7806 & 0,7816 & 0,7816 & 0,7817 \\
0,4 & 0,5971 & 0,5979 & 0,5996 & 0,5996 & 0,5997 & 0,5996 & 0,5997 \\
0,6 & 0,3997 & 0,4000 & 0,4000 & 0,4000 & 0,4000 & 0,4000 & 0,4000 \\
0,8 & 0,2015 & 0,2021 & 0,2010 & 0,2009 & 0,2009 & 0,2009 & 0,2009 \\
1,0 & 0,0499 & 0,0469 & 0,0500 & 0,0496 & 0,0500 & 0,0499 & 0,0500 \\
\hline
\end{tabular}

Tabela 3.4: Esforço cortante no pórtico - $K_{r}=20$, família $\Im_{N}^{k=0, p=2}$ 


\begin{tabular}{|c|cc|cc|cc|c|}
\hline Posição & \multicolumn{2}{|c|}{ 6 nós } & \multicolumn{2}{c|}{ 11 nós } & \multicolumn{2}{c|}{ 21 nós } & Solução \\
\cline { 2 - 7 } z & Nuvens & MEF & Nuvens & MEF & Nuvens & MEF & analítica \\
\hline 0,0 & 0,0425 & 0,0295 & 0,0464 & 0,0393 & 0,0474 & 0,0446 & 0,0475 \\
0,2 & 0,0000 & $-0,0010$ & $-0,0013$ & $-0,0016$ & $-0,0015$ & $-0,0016$ & $-0,0016$ \\
0,4 & $-0,0021$ & $-0,0024$ & $-0,0025$ & $-0,0024$ & $-0,0025$ & $-0,0024$ & $-0,0025$ \\
0,6 & $-0,0024$ & $-0,0025$ & $-0,0025$ & $-0,0024$ & $-0,0025$ & $-0,0024$ & $-0,0025$ \\
0,8 & $-0,0024$ & $-0,0028$ & $-0,0024$ & $-0,0024$ & $-0,0025$ & $-0,0024$ & $-0,0025$ \\
1,0 & $-0,0003$ & $-0,0009$ & $-0,0001$ & $-0,0004$ & 0,0000 & $-0,0001$ & 0,0000 \\
\hline
\end{tabular}

Tabela 3.5: Esforço momento fletor na parede $-K_{r}=20$, família $\Im_{N}^{k=0, p=2}$

\subsection{Problemas de Elasticidade Linear Estática - Aná- lise Bi-Dimensional pelo MEFG}

Todos os problemas analisados nesta seção são regidos pelas equações da elasticidade linear estática (pequenas deformações e deslocamentos), variando-se, é claro, a geometria, material e condições de contorno. Uma resumida formulação é apresentada a seguir, visando-se chegar ao problema variacional aproximado por Galerkin. Maiores detalhes com relação às equações da elasticidade podem ser encontrados, por exemplo, em MALVERN (1969) e TIMOSHENKO; GOODIER (1980).

Seja, então, o seguinte PVC que exprime, em forma forte e em função dos deslocamentos, o equilíbrio em um ponto genérico do domínio aberto e limitado $\Omega \in \mathbb{R}^{2}$ :

$$
\text { Encontrar } \boldsymbol{u} \text { tal que: }\left\{\begin{array}{cccc|}
\boldsymbol{\nabla}^{T} \boldsymbol{\Sigma}(\boldsymbol{u})+\boldsymbol{b} & =\mathbf{0} & \text { em } \Omega & \\
\boldsymbol{u} & =\hat{\boldsymbol{u}} & & \mathrm{em} \Gamma_{D} \\
\boldsymbol{t}(\boldsymbol{u}) & =\hat{\boldsymbol{t}} & & \mathrm{em} \Gamma_{N}
\end{array}\right.
$$

sendo:

- $\boldsymbol{u}^{T} \stackrel{\text { def }}{=}\left[\begin{array}{ll}u_{x} & u_{y}\end{array}\right]$ o vetor das componentes de deslocamentos nas direções dos eixos $x$ e $y$;

- $\Gamma_{D}$ região do contorno em que as condições de Dirichlet são definidas;

- $\Gamma_{N}$ região do contorno em que as condições de Neumman são definidas;

- $\Sigma$ tensor de tensões de segunda ordem;

- $\boldsymbol{\nabla}^{T}=\left[\begin{array}{cc}\frac{\partial}{\partial x_{1}} & \frac{\partial}{\partial x_{2}}\end{array}\right]$ 
- $\boldsymbol{b}$ força de volume;

- $\boldsymbol{t}=\boldsymbol{\Sigma} \boldsymbol{n}$, vetor de forças superficiais no contorno $\partial \Omega$;

- $\hat{\boldsymbol{u}}$ e $\hat{\boldsymbol{t}}$ vetores de deslocamento e tração prescritos;

- $\boldsymbol{n}$ versor que descreve a orientação do contorno $\partial \Omega$.

A correspondente forma variacional pode, então, ser descrita como:

$$
\text { Encontrar } \boldsymbol{u} \in \mathcal{H}^{1} \text { tal que: } \mathcal{B}(\boldsymbol{u}, \boldsymbol{v})=l(\boldsymbol{v}) \forall \boldsymbol{v} \in \mathcal{H}^{1}
$$

onde:

- $\mathcal{H}^{1}$ é o espaço de Hilbert de ordem $1^{3}$;

- os operadores variacionais são definidos como:

$$
\begin{gathered}
\mathcal{B}(\boldsymbol{u}, \boldsymbol{v})=\iint_{\Omega} \varepsilon^{T}(\boldsymbol{v}) \boldsymbol{\sigma}(\boldsymbol{u}) l_{z} d x d y \\
l(\boldsymbol{v})=\iint_{\Omega} \boldsymbol{v}^{T} \boldsymbol{b} l_{z} d x d y+\int_{\Gamma_{N}} \boldsymbol{v}^{T} \hat{\boldsymbol{t}} l_{z} d s
\end{gathered}
$$

- $\boldsymbol{v}^{T} \stackrel{\text { def }}{=}\left[\begin{array}{ll}v_{x} & v_{y}\end{array}\right]$ é o vetor das funções teste de deslocamentos em $x$ e $y$;

- $\boldsymbol{\sigma} \stackrel{\text { def }}{=}\left\{\begin{array}{lll}\sigma_{x} & \sigma_{y} & \sigma_{x y}\end{array}\right\}$ e $\boldsymbol{\varepsilon} \stackrel{\text { def }}{=}\left\{\begin{array}{lll}\varepsilon_{x} & \varepsilon_{y} & \gamma_{x y}\end{array}\right\}$ são representações vetoriais compactas dos campos de tensão e deformação, cuja relação $\sigma=C \varepsilon$ é estabelecida pela matriz de propriedades constitutivas elásticas $C$;

- $l_{z}$ a dimensão do corpo elástico na direção $z$ aqui tomada como constante.

A aproximação de Galerkin, através das funções do MEFG, corresponde à solução do seguinte problema, projetada no sub-espaço de dimensão finita $\tilde{X}$ :

$$
\text { Encontrar } \tilde{\boldsymbol{u}} \in \tilde{X} \text { tal que: } \mathcal{B}(\tilde{\boldsymbol{u}}, \tilde{\boldsymbol{v}})=l(\tilde{\boldsymbol{v}}) \forall \tilde{\boldsymbol{v}} \in \tilde{X}
$$

em que $\tilde{\boldsymbol{u}}$ e $\tilde{\boldsymbol{v}}$ são definidas, cada uma, a partir da expressão (2.31), para $\mathbb{R}^{2}$, ou seja:

\footnotetext{
${ }^{3} \mathrm{Na}$ forma variacional do PVC da elasticidade, a máxima derivada presente é de ordem 1. Torna-se, portanto, necessário que a aproximação e sua primeira derivada (em $x$ e $y$ ) sejam, no mínimo, integráveis no domínio $\Omega$.
} 


$$
\begin{aligned}
& \tilde{\boldsymbol{u}}(\boldsymbol{x})=\sum_{j=1}^{N} \mathcal{N}_{j}(\boldsymbol{x})\left\{\boldsymbol{u}_{j}+\sum_{i=1}^{q_{j}} L_{j i}(\boldsymbol{x}) \boldsymbol{b}_{j i}\right\} \Rightarrow \tilde{\boldsymbol{u}}=\boldsymbol{\Phi}^{T} \boldsymbol{U} \\
& \tilde{\boldsymbol{v}}(\boldsymbol{x})=\sum_{j=1}^{N} \mathcal{N}_{j}(\boldsymbol{x})\left\{\boldsymbol{v}_{j}+\sum_{i=1}^{q_{j}} L_{j i}(\boldsymbol{x}) \boldsymbol{c}_{j i}\right\} \Rightarrow \tilde{\boldsymbol{v}}=\boldsymbol{\Phi}^{T} \boldsymbol{V}
\end{aligned}
$$

Nas relações anteriores foram empregadas as seguintes representações para os deslocamentos, funções de teste e funções de aproximação:

$$
\begin{aligned}
& \boldsymbol{U}^{T} \stackrel{\text { def }}{=}\left[\begin{array}{llllllllllll}
u_{1}^{x} & u_{1}^{y} & b_{11}^{x} & b_{11}^{y} & \cdots & b_{1 q_{1}}^{x} & b_{1 q_{1}}^{y} & \cdots & u_{N}^{x} & u_{N}^{y} \cdots & b_{N q_{N}}^{x} & b_{N q_{N}}^{y}
\end{array}\right](3 \\
& \boldsymbol{V}^{T} \stackrel{\text { def }}{=}\left[\begin{array}{llllllllllll}
v_{1}^{x} & v_{1}^{y} & c_{11}^{x} & c_{11}^{y} & \cdots & c_{1 q_{1}}^{x} & c_{1 q_{1}}^{y} & \cdots & v_{N}^{x} & v_{N}^{y} \cdots & c_{N q_{N}}^{x} & c_{N q_{N}}^{y}
\end{array}\right] \\
& \boldsymbol{\Phi}^{T} \stackrel{\text { def }}{=}\left[\begin{array}{ccccccccc}
\mathcal{N}_{1} & 0 & L_{11} \mathcal{N}_{1} & 0 & \cdots & 0 & L_{1 q_{1}} \mathcal{N}_{1} & 0 & \cdots \\
0 & \mathcal{N}_{1} & 0 & L_{11} \mathcal{N}_{1} & 0 & \ldots & 0 & L_{1 q_{1}} \mathcal{N}_{1} & 0
\end{array}\right. \\
& \begin{array}{lllllllll}
0 & \mathcal{N}_{N} & 0 & L_{N 1} \mathcal{N}_{N} & 0 & \cdots & 0 & L_{N q_{N}} \mathcal{N}_{N} & 0
\end{array} \\
& \left.\begin{array}{lllllllll}
\cdots & 0 & \mathcal{N}_{N} & 0 & L_{N 1} \mathcal{N}_{N} & 0 & \ldots & 0 & L_{N q_{N}} \mathcal{N}_{N}
\end{array}\right]
\end{aligned}
$$

Para o caso em que estiverem sendo usadas as seguintes funções aproximadora e de teste:

$$
\begin{aligned}
& \boldsymbol{u}_{p}(\boldsymbol{x})=\sum_{j=1}^{N} \mathcal{N}_{j}(\boldsymbol{x})\left\{\boldsymbol{u}_{j}+\sum_{i=1}^{q_{j}(p)} p_{j i}(\boldsymbol{x}) \boldsymbol{b}_{j i}\right\} \Rightarrow \boldsymbol{u}=\boldsymbol{\Phi}^{T} \boldsymbol{U} \\
& \boldsymbol{v}_{p}(\boldsymbol{x})=\sum_{j=1}^{N} \mathcal{N}_{j}(\boldsymbol{x})\left\{\boldsymbol{v}_{j}+\sum_{i=1}^{q_{j}(p)} p_{j i}(\boldsymbol{x}) \boldsymbol{c}_{j i}\right\} \Rightarrow \boldsymbol{v}=\boldsymbol{\Phi}^{T} \boldsymbol{V}
\end{aligned}
$$

o espaço $\tilde{X}$ é substituído por $\mathcal{X}_{p} \stackrel{\text { def }}{=}\left\{\boldsymbol{u}_{p} \in \mathcal{H}^{1} ;\left.\boldsymbol{u}_{p}\right|_{\mathcal{K}} \in \mathcal{P}_{p}, \mathcal{K} \in \Omega\right\}$, onde $\left.\boldsymbol{u}_{p}\right|_{\mathcal{K}}$ referese à restrição da função $\boldsymbol{u}_{p}$ ao elemento finito $\mathcal{K}$ pertencente ao domínio $\Omega$. Definese, então, a seguinte matriz das funções de aproximação, em que a PU $\left\{\mathcal{N}_{j}\right\}_{j=1}^{N}$ é enriquecida pelos monômios $p_{j i}$ de (2.15): 


$$
\begin{array}{ccccccccccc}
\boldsymbol{\Phi}^{T} \stackrel{\text { def }}{=} & {\left[\begin{array}{ccccccccc}
\mathcal{N}_{1} & 0 & p_{11} \mathcal{N}_{1} & 0 & \ldots & 0 & p_{1 q_{1}(p)} \mathcal{N}_{1} & 0 & \ldots \\
0 & \mathcal{N}_{1} & 0 & p_{11} \mathcal{N}_{1} & 0 & \ldots & 0 & p_{1 q_{1}(p)} \mathcal{N}_{1} & 0 \\
0 & \mathcal{N}_{N} & 0 & p_{N 1} \mathcal{N}_{N} & 0 & \ldots & 0 & p_{N q_{N}(p)} \mathcal{N}_{N} & 0 \\
\ldots & 0 & \mathcal{N}_{N} & 0 & p_{N 1} \mathcal{N}_{N} & 0 & \ldots & 0 & p_{N q_{N}(p)} \mathcal{N}_{N}
\end{array}\right]}
\end{array}
$$

Introduzindo-se, então, a matriz de operadores diferenciais que determina o campo de deformações a partir dos deslocamentos:

$$
\boldsymbol{L} \stackrel{\text { def }}{=}\left[\begin{array}{cc}
\partial / \partial x & 0 \\
0 & \partial / \partial y \\
\partial / \partial y & \partial / \partial x
\end{array}\right]
$$

e o operador que relaciona os deslocamentos generalizados $U$ às deformações $\varepsilon$ :

$$
\boldsymbol{B}=\boldsymbol{L} \boldsymbol{\Phi}^{T}
$$

pode-se chegar, após algum algebrismo, à forma final para o sistema de equações a ser resolvido:

$$
\boldsymbol{K} \boldsymbol{U}=\boldsymbol{F}
$$

onde:

$$
\begin{gathered}
\boldsymbol{K}=\iint_{\Omega} \boldsymbol{B}^{T} \boldsymbol{C} \boldsymbol{B} l_{z} d x d y \\
\boldsymbol{F}=\iint_{\Omega} \boldsymbol{\Phi} \boldsymbol{b} l_{z} d x d y+\int_{\Gamma_{N}} \boldsymbol{\Phi} \hat{\boldsymbol{t}} l_{z} d s
\end{gathered}
$$

Nos problemas apresentados a seguir, o sistema de equações (3.23) foi resolvido pelo procedimento de Babuška, apêndice $\mathrm{C}$, com perturbação $\epsilon=10^{-12}$ na diagonal principal de $\boldsymbol{K}$ e tolerância $T O L=10^{-10}$. Resultados semelhantes, a menos de algum erro de precisão numérica, foram obtidos para testes realizados com o pacote MA27, DUFF; REID (1983), que implementa um método direto de solução conforme se discute no apêndice $\mathrm{C}$. 


\subsubsection{Efeito da Distorção da Malha de Elementos}

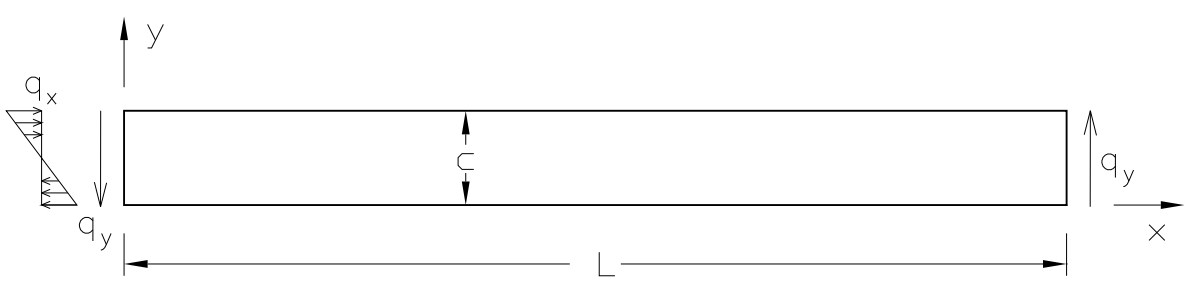

(a) $L=100, c=10$

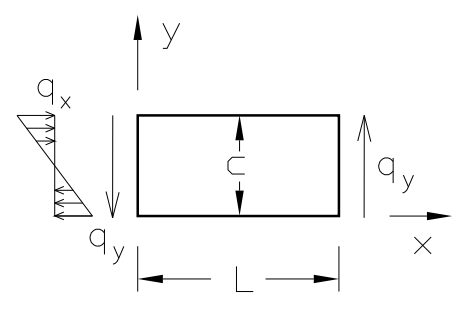

(b) $L=20, c=10$

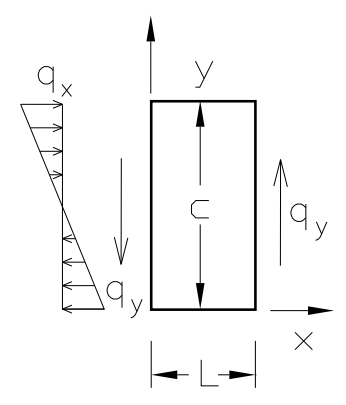

(c) $L=10, c=20$

Figura 3.4: Geometria das estruturas propostas - sem unidades

Neste exemplo, procura-se averiguar a influência da distorção dos elementos na qualidade da aproximação via MEFG. Os problemas propostos são os mesmos apresentados em LEE; BATHE (1993), correspondendo a uma viga submetida a uma força aplicada em sua extremidade, como uma carga distribuída do tipo parabólica:

$$
q_{y}=\frac{120 y}{L}-\frac{120 y^{2}}{c L}
$$

Para que o problema em estado plano de tensões simule o comportamento de uma viga engastada, também as reações de apoio são impostas, como condições de contorno naturais, aplicando-se na face $x=0$ a reação de cisalhamento no valor de $-q_{y}$ e de flexão através da distribuição:

$$
q_{x}=\frac{240 y}{c}-120
$$

A estrutura encontra-se, portanto, auto-equilibrada e os vínculos introduzidos servem apenas para eliminar os movimentos de corpo rígido.

Três geometrias diferentes, parametrizadas segundo $L$ e $c$, são analisadas conforme é ilustrado nas Figuras 3.4(a), 3.4(b) e 3.4(c). Os dados para as propriedades 
do material são apresentados a seguir, sem a adoção de unidades, conforme aparecem em LEE; BATHE (1993):

- Módulo de Elasticidade $E=1,0 \cdot 10^{7}$;

- coeficiente de Poisson $\nu=0,3$;

- espessura $t=1,0$;

Também em LEE; BATHE (1993) são fornecidas as seguintes expressões analíticas para as funções de deslocamentos:

$$
\begin{aligned}
& u_{x}=\frac{1}{E}\left(\frac{120}{c L} x^{2} y-\frac{92}{c L} y^{3}-\frac{60}{L} x^{2}-\frac{240}{c} x y+\frac{138}{L} y^{2}+120 x-\frac{46 c}{L} y\right) \\
& u_{y}=\frac{1}{E}\left(-\frac{40}{c L} x^{3}-\frac{36}{c L} x y^{2}+\frac{120}{c} x^{2}+\frac{36}{L} x y+\frac{36}{c} y^{2}+\frac{46 c}{L} x-36 y\right)
\end{aligned}
$$

Para as três geometrias, diversos tipos de malha são propostas, como mostram as Figuras 3.5(a) até 3.5(f), através das quais são estudados os efeitos de dois tipos de distorção:

- angular - malhas das Figuras 3.5(a), 3.5(b) e 3.5(c);

- devido à curvatura de uma face do elemento - malhas das Figuras 3.5(d), 3.5(e), $3.5(\mathrm{f})$ e $3.5(\mathrm{~g})$.

Nas análises realizadas com o MEFG através das malhas V e VII foi empregado, para descrever a geometria curva da face dos elementos, o método das "funções de mistura" (blending function method). Esta estratégia bastante utilizada na versão $p$ do MEF, é proposta por GORDON; HALL (1973) e permitinde a representação de diferentes tipos de contornos sem que seja necessária a utilização de uma grande quantidade de elementos. Basicamente, consiste em se introduzir no mapeamento das coordenadas físicas dos elementos com relação ao elemento mestre uma expressão para a forma curva considerada.

O emprego das "funções de mistura" torna o integrando de (3.24) não-polinomial e, por isso, a integração numérica é realizada com $5 \times 5$ pontos de Gauss-Legendre, no caso das análises com as malhas V e VII. Para as demais análises foram adotados $3 \times 3$ pontos para a quadratura numérica.

Na Tabela 3.6, encontram-se os resultados obtidos através das análises feitas com o MEFG e os respectivos graus de liberdade (NGL) antes de se considerar os vínculos nodais. Estes resultados são confrontados com os apresentados em LEE; BATHE 


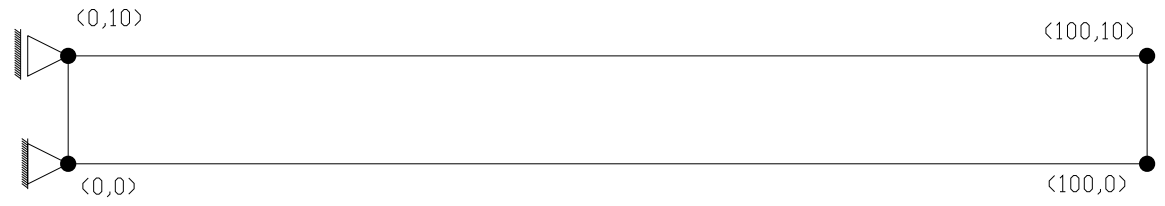

(a) Malha I

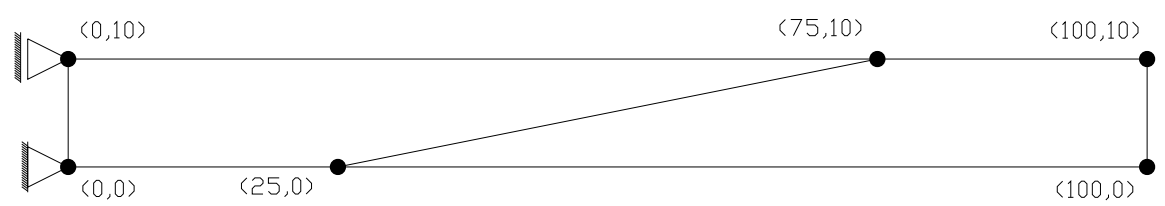

(b) Malha II

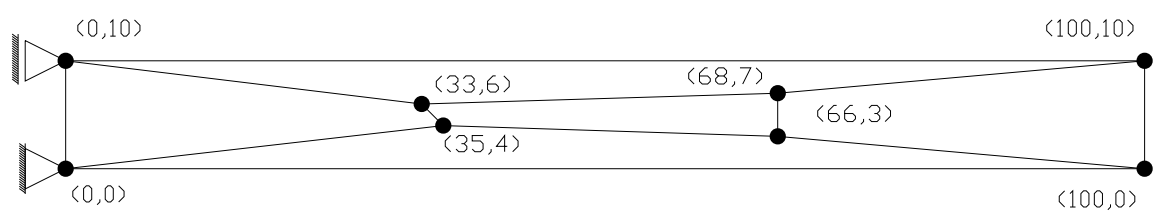

(c) Malha III

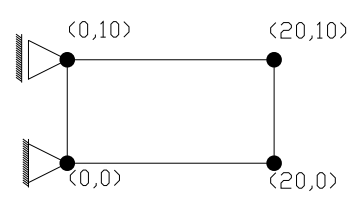

(d) Malha IV

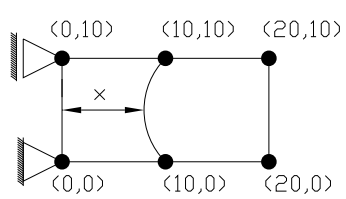

(e) Malha V $x(y)=10-\frac{8 y}{10}\left(1-\frac{y}{10}\right)$

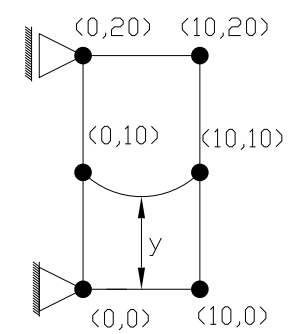

(g) Malha VII

$$
y(x)=10-\frac{8 x}{10}\left(1-\frac{x}{10}\right)
$$

Figura 3.5: Discretizações propostas 
(1993) para o MEF isoparamétrico, com interpolações cúbicas Serendípeta e Lagrangiana. A solução analítica para o problema da Figura 3.4(a) é $u_{y}(100,0)=8,046 \cdot 10^{-3}$, para o da Figura 3.4(b) é $u_{y}(20,0)=3,660 \cdot 10^{-4}$ e para o problema da Figura 3.4(c) é $u_{y}(10,0)=1,320 \cdot 10^{-4}$.

Para que somente aos efeitos da distorção sejam considerados, a aproximação adotada para o MEFG deve representar exatamente as soluções (3.28) e (3.29) com um único elemento não distorcido, malhas I, IV e VI (o mesmo ocorre com as interpolações Serendípeta e Lagrangiana, ambas cúbicas). Para isso, a PU definida pelas funções Lagrangianas bi-lineares, é enriquecida, como estabelecido em (3.19) e (3.20), em que o vetor de funções de forma associado a cada nó $\boldsymbol{x}_{j}$ é dado por:

$$
\begin{aligned}
\boldsymbol{\Phi}_{j}^{T} \stackrel{\text { def }}{=} & {\left[\begin{array}{cccccc}
\mathcal{N}_{j} & 0 & \frac{x-x_{j}}{h_{j}} \mathcal{N}_{j} & 0 & \frac{y-y_{j}}{h_{j}} \mathcal{N}_{j} & 0 \\
0 & \mathcal{N}_{j} & 0 & \frac{x-x_{j}}{h_{j}} \mathcal{N}_{j} & 0 & \frac{y-y_{j}}{h_{j}} \mathcal{N}_{j} \\
& \left(\frac{x-x_{j}}{h_{j}}\right)^{2} \mathcal{N}_{j} & 0 & \left(\frac{y-y_{j}}{h_{j}}\right)^{2} & \mathcal{N}_{j} & 0 \\
0 & \left(\frac{x-x_{j}}{h_{j}}\right)^{2} \mathcal{N}_{j} & 0 & \left(\frac{y-y_{j}}{h_{j}}\right)^{2} \mathcal{N}_{j}
\end{array}\right] }
\end{aligned}
$$

sendo $h_{j}$ adotado como a maior distância entre o nó $\boldsymbol{x}_{j}$ e os demais nós pertencentes à nuvem $\omega_{j}{ }^{4}$. Com tal enriquecimento, as funções de aproximação $\Phi$ resultantes geram um espaço de polinômios equivalente ao obtido com as aproximações Serendípetas cúbicas (em elemento regular), ou seja, o espaço $\mathcal{P}_{3}$ conforme se descreve no apêndice D.

\begin{tabular}{|c|c|c|c|c|c|c|}
\hline \multirow{2}{*}{ Malha } & \multicolumn{2}{|c|}{$\begin{array}{c}\text { Interpolação Serendípeta } \\
\text { 12 nós }\end{array}$} & $\begin{array}{c}\text { Interpolação Lagrangiana } \\
16 \text { nós }\end{array}$ & \multicolumn{2}{|c|}{$\begin{array}{c}\text { MEFG } \\
\text { 4 nós }\end{array}$} \\
\cline { 2 - 7 } & NGL & $u_{y}$ & NGL & $u_{y}$ & NGL & $u_{y}$ \\
\hline \hline 1 & 24 & $8,046 \cdot 10^{-3}$ & 32 & $8,046 \cdot 10^{-3}$ & 40 & $8,046 \cdot 10^{-3}$ \\
\hline 2 & 40 & $3,444 \cdot 10^{-3}$ & 56 & $8,046 \cdot 10^{-3}$ & 60 & $8,046 \cdot 10^{-3}$ \\
\hline 3 & 64 & $0,585 \cdot 10^{-3}$ & 104 & $8,046 \cdot 10^{-3}$ & 80 & $8,046 \cdot 10^{-3}$ \\
\hline 4 & 24 & $3,660 \cdot 10^{-4}$ & 32 & $3,660 \cdot 10^{-4}$ & 40 & $3,660 \cdot 10^{-4}$ \\
\hline 5 & 40 & $3,534 \cdot 10^{-4}$ & 56 & $3,659 \cdot 10^{-4}$ & 60 & $3,653 \cdot 10^{-4}$ \\
\hline 6 & 24 & $1,320 \cdot 10^{-4}$ & 32 & $1,320 \cdot 10^{-4}$ & 40 & $1,320 \cdot 10^{-4}$ \\
\hline 7 & 36 & $1,320 \cdot 10^{-4}$ & 56 & $1,314 \cdot 10^{-4}$ & 60 & $1,306 \cdot 10^{-4}$ \\
\hline
\end{tabular}

Tabela 3.6: Resultados para o MEF de LEE; BATHE (1993) e MEFG

\footnotetext{
${ }^{4}$ Outro procedimento poderia ser adotado para se definir $h_{j}$, inclusive definindo-se valores diferentes nas direções $x$ e $y$, como é sugerido na expressão (2.15) para o Método das Nuvens $h p$
} 
Os resultados obtidos permitem mostrar o excelente desempenho das aproximações construídas pelo MEFG. Assim como no MEF com interpolação Lagrangiana, no MEFG a distorção angular não compromete a aproximação, obtendo-se, exatamente, a solução analítica. A presença de elementos com faces curvas perturba um pouco os resultados, mas ainda assim podem ser considerados bastante bons, especialmente se comparados aos obtidos para o caso da interpolação Serendípeta.

Em BATHE (1996), mostra-se que os elementos Serendípetos com 12 nós ao apresentar distorção angular deixam de representar exatamente polinômios completos de grau 3, restringindo-se, apenas, aos polinômios lineares. Esta deficiência resulta do mapeamento das funções de forma que são definidas para o elemento mestre, quadrangular, devendo ser mapeadas para as coordenas físicas do problema. Devido à distorção angular dos elementos empregados na discretização, o mapeamento deixa de ser linear e a aproximação é empobrecida ao ser descrita nas coordenadas físicas. Com os elementos Lagrangianos de 16 nós, também ocorre uma penalização com relação à capacidade de reproduzir os mesmos polinômios originalmente representados no elemento mestre. A dimensão do espaço definido pelas interpolações Lagrangianas é, contudo, bem superior à dimensão do espaço das interpolações Serendípetas. Por essa razão, a penalização proveniente da distorção angular não chega a impedir que as aproximações sejam capazes de representar exatamente polinômios completos até o terceiro grau. Explica-se, assim, diferença dos resultados obtidos para as duas formas de aproximação com o MEF. No MEFG, apenas as funções bi-lineares da PU são construídas no elemento mestre. As funções utilizadas para o enriquecimento da aproximação, (2.15), são descritas diretamente nas coordenadas físicas do problema, permitindo-se que a capacidade de representação de polinômios completos até o grau 3 não seja penalizada com o mapeamento.

O polinômio completo de maior grau que a aproximação pode representar de forma exata determina, para problemas de solução suave, a taxa de convergência do método, SZABÓ; BABUŠKA (1991). Como no MEFG apesar da distorção da malha ainda assim a aproximação continuou a produzir de forma exata polinômios cúbicos completos é de se esperar que as aproximações não tenham a convergência perturbada. Adquire-se, portanto, uma certa independência da malha de elementos graças à estratégia de enriquecimento. Esta observação deve ser, contudo, considerada com bastante cautela. A independência total da malha encontrada neste exemplo para o caso da distorção angular, deve-se ao fato de que a solução exata também é polino- 
mial. Por essa razão, como a aproximação do MEFG utilizada continua reproduzindo exatamente polinômios cúbicos completos, seja qual for o grau de distorção angular dos elementos, obtém-se a própria solução exata. Entretanto, se os polinômios reproduzidos pela aproximação não são capazes de representar a resposta do problema, o erro encontrado pode ser ampliado em função do grau de distorção da malha. Para explicar esse fato deve-se recorrer às estimativas de erro "a priori” da aproximação com o MEFG, discutidas no apêndice E. A taxa de convergência, contudo, não é penalizada pois, como já comentado, não há perda de termos dos polinômios representados pelas funções de forma enriquecidas. A independência com relação à malha no MEFG deve, portanto, ser relativizada e interpretada como se referida à sua taxa de convergência.

Por último, algum comentário deve ser registrado com relação ao número de graus de liberdade exigido nas análises realizadas com as três primeiras malhas. Para um único elemento, malha I, o número de funções de forma do MEFG é superior ao utilizado no MEF com interpolação Lagrangiana, enquanto que essa situação invertese para a malha III. A explicação é bastante simples e está relacionada à maneira com que as funções de forma são definidas no MEFG, associadas exclusivamente aos nós. Sendo assim, cada grau de liberdade é compartilhado por todos os elementos pertencentes à nuvem correspondente. No MEF com interpolação Lagrangiana por sua vez, os nós internos pertencem a um único elemento e os nós de face são compartilhados por dois elementos no máximo. Este fato pode vir a compensar a maior quantidade de funções definidas para um elemento no MEFG, fazendo com que, no somatório geral em malhas de vários elementos, as aproximações possam ser descritas com um menor NGL do que no MEF. Este fato depende é, claro, do número de elementos por nuvem. Em análises tri-dimensionais com elementos tetraédricos, por exemplo, esse efeito é mais acentuado, como se observa em DUARTE; BABUŠKA; ODEN (2000).

\subsubsection{Chapa com Orifício}

A chapa, cuja a geometria é mostrada na Figura 3.6(a) encontra-se submetida a uma tensão uniforme $\sigma_{\infty}$ a uma distância razoavelmente grande do seu orifício. A análise via MEFG foi realizada para o domínio $A B C D E$, Figura 3.6(b), para um quarto da região correspondente à vizinhança do orifício. As condições de contorno essenciais reproduzem a simetria do problema. Para sua imposição é necessário que, não apenas os deslocamentos nodais, como também os parâmetros das funções enriquecidas que contribuem para o deslocamento ao longo das faces dos elementos coincidentes com 
o contorno de Dirichlet sejam anulados. Por sua vez, as condições de contorno de Neumman são representadas pela seguinte distribuição de tensões, obtida da solução elástica clássica, TIMOSHENKO; GOODIER (1980):

$$
\begin{aligned}
\sigma_{x} & =\sigma_{\infty}\left[1-\frac{a^{2}}{r^{2}}\left(\frac{3}{2} \cos 2 \theta+\cos 4 \theta\right)+\frac{3 a^{4}}{2 r^{4}} \cos 4 \theta\right] \\
\sigma_{y} & =\sigma_{\infty}\left[-\frac{a^{2}}{r^{2}}\left(\frac{1}{2} \cos 2 \theta-\cos 4 \theta\right)-\frac{3 a^{4}}{2 r^{4}} \cos 4 \theta\right] \\
\tau_{x y} & =\sigma_{\infty}\left[-\frac{a^{2}}{r^{2}}\left(\frac{1}{2} \operatorname{sen} 2 \theta+\cos 4 \theta\right)+\frac{3 a^{4}}{2 r^{4}} \cos 4 \theta\right]
\end{aligned}
$$

que produz o campo de deslocamentos descrito a seguir:

$$
\begin{aligned}
& \left.u_{x}=\frac{\sigma_{\infty} a}{8 G}\left\{\frac{r}{a}(\kappa+1) \cos \theta+\frac{2 a}{r}[(1+\kappa) \cos \theta+\cos 3 \theta]-\frac{2 a^{3}}{r^{3}} \cos 3 \theta\right\}(3.3) \operatorname{sen} \theta+\frac{2 a}{r}[(1-\kappa) \operatorname{sen} \theta+\operatorname{sen} 3 \theta]-\frac{2 a^{3}}{r^{3}} \operatorname{sen} 3 \theta\right\} \\
& u_{y}=\frac{\sigma_{\infty} a}{8 G}\left\{\frac{r}{a}(\kappa-3)\right.
\end{aligned}
$$

onde $\kappa$ é um parâmetro que depende do coeficiente de Poisson e é definido como:

$$
\kappa \stackrel{\text { def }}{=}\left\{\begin{array}{ccc}
3-4 \nu & \text { para } & \text { Estado plano de deformação } \\
\frac{3-\nu}{1+\nu} & \text { para } & \text { Estado plano de tensão }
\end{array}\right.
$$

e $G$ é o módulo de elasticidade transversal:

$$
G=\frac{E}{2(1+\nu)}
$$

Para a análise foi considerado o estado plano de deformação com $\nu=0,3$ e $E=1,0$

\begin{tabular}{|c|c|c|c|c|c|c|}
\hline \multirow{2}{*}{$\mathrm{p}$} & \multicolumn{3}{|c|}{ MEF,SZABÓ; BABUŠKA (1991) } & \multicolumn{3}{c|}{ MEFG } \\
\cline { 2 - 7 } & NGL & $\mathcal{U}_{p} E / \sigma_{\infty}^{2} a^{2} t$ & $\mathcal{E}_{\%}(\%)$ & NGL & $\mathcal{U}_{p} E / \sigma_{\infty}^{2} a^{2} t$ & $\mathcal{E}_{\%}(\%)$ \\
\hline \hline 1 & 8 & 7,36762 & 20,59 & 8 & 7,36763 & 20,59 \\
\hline 2 & 20 & 7,54738 & 13,79 & 28 & 7,55896 & 13,23 \\
\hline 3 & 32 & 7,62008 & 9,78 & 48 & 7,63790 & 8,51 \\
\hline 4 & 48 & 7,65904 & 6,71 & 80 & 7,67227 & 5,27 \\
\hline 5 & 68 & 7,67875 & 4,40 & 124 & 7,68941 & 2,35 \\
\hline 6 & 92 & 7,68805 & 2,70 & 180 & 7,69229 & 1,33 \\
\hline 7 & 120 & 7,69165 & 1,61 & - & - & - \\
\hline 8 & 152 & 7,69295 & 0,95 & - & - & - \\
\hline
\end{tabular}

Tabela 3.7: Resultados comparativos para a energia de deformação normalizada, entre o MEF, SZABÓ; BABUŠKA (1991) e o MEFG, $\left(\mathcal{U}_{p} E / \sigma_{\infty}^{2} a^{2} t\right)$. Solução analítica exata normalizada de SZABÓ; BABUŠKA (1991) $U E / \sigma_{\infty}^{2} a^{2} t=7,69365$. 


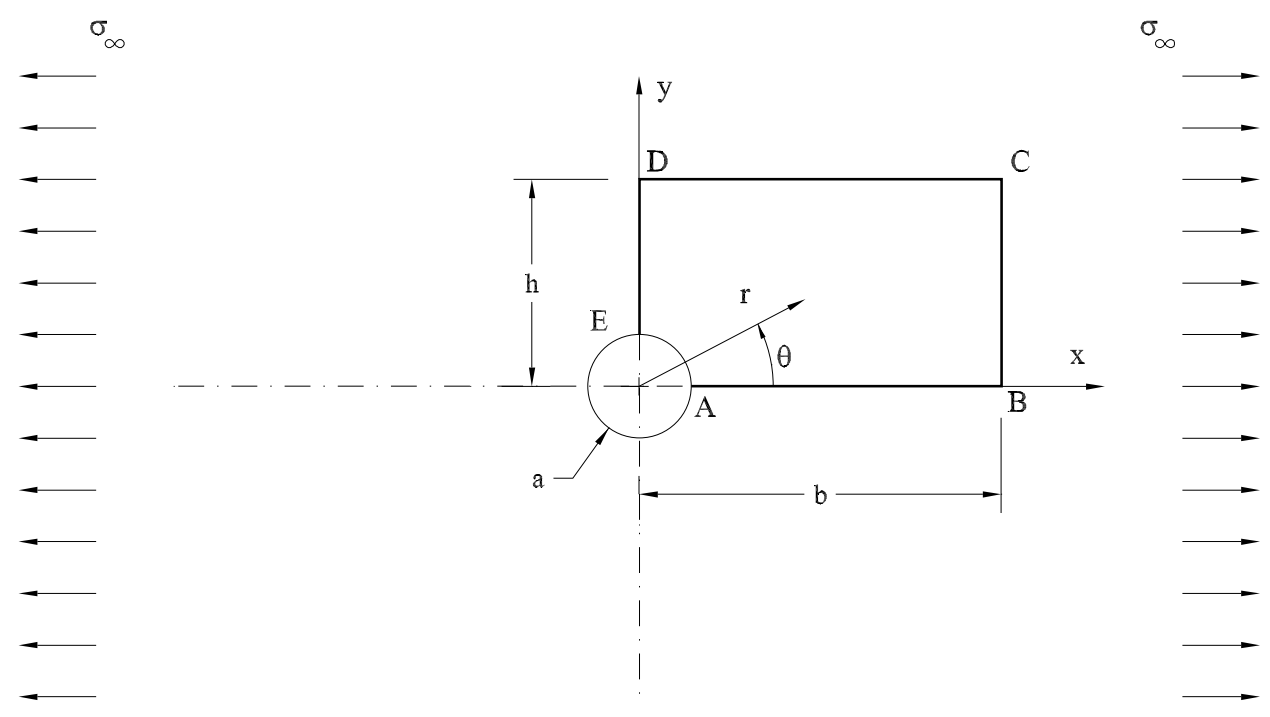

(a) Geometria

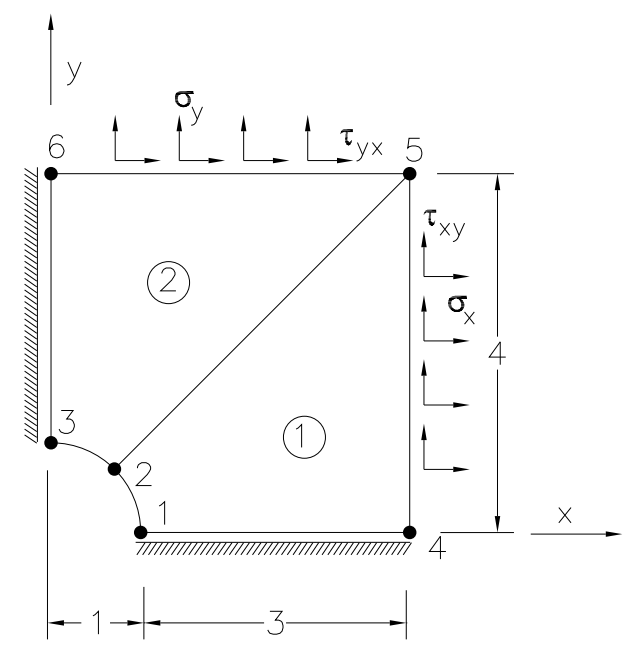

(b) Discretização

Figura 3.6: Chapa com orifício 
A malha de elementos da Figura 3.6(b) foi empregada para definir a PU, sobre a qual foram realizados diversos níveis de enriquecimento polinomial $p$, de tal maneira que a aproximação $\boldsymbol{u}_{p}$ obtida, conforme representação em (3.19), gerasse o espaço $\mathcal{P}_{p}$. No que se refere à curvatura do contorno $E A$, essa foi considerada empregando-se, para o mapeamento, o método das "funções de mistura".

$\mathrm{Na}$ Tabela 3.7, os resultados para energia de deformação aproximada $\mathcal{U}_{p}$, obtidos com o MEFG, são contrapostos aos obtidos em SZABÓ; BABUŠKA (1991) com o MEF hierárquico. Cada linha da Tabela corresponde a uma diferente ordem de aproximação $p$ do espaço $\mathcal{P}_{p}$. NGL corresponde, neste exemplo, ao número de graus de liberdade da discretização após a imposição dos vínculos nodais. Na coluna $\mathcal{E}_{\%}$, encontram-se os erros relativos da solução aproximada de cada análise, definidos como:

$$
\mathcal{E}_{\%} \stackrel{\text { def }}{=} \frac{\|e\|_{u}}{\|u\|_{u}} 100 \%
$$

onde são utilizadas as seguintes normas de energia:

$$
\begin{gathered}
\|e\|_{u} \stackrel{\text { def }}{=} \sqrt{\mathcal{B}\left(\boldsymbol{u}-\boldsymbol{u}_{p}, \boldsymbol{u}-\boldsymbol{u}_{p}\right)}=\sqrt{2 \mathcal{U}-2 \mathcal{U}_{p}} \\
\|u\|_{u} \stackrel{\text { def }}{=} \sqrt{\mathcal{B}(\boldsymbol{u}, \boldsymbol{u})}=\sqrt{2 \mathcal{U}}
\end{gathered}
$$

Para comparar a convergência de ambos os métodos, são utilizados dois gráficos em que o erro relativo é contraposto à ordem polinomial, Figura 3.7(a), e ao número de graus de liberdade, Figura 3.7(b). Nota-se uma razoável equivalência entre as análises. Este fato já era esperado, pois, também no MEF hierárquico, representa-se para cada ordem $p$ o mesmo espaço polinomial do tipo $\mathcal{P}_{p}$ representado no MEFG. Dado o grande número de graus de liberdade redundantes introduzidos pelo MEFG, seu desempenho mostrou-se levemente inferior no gráfico da Figura 3.7(b) . Tal diferença, contudo, é muito pequena, se comparada às vantagens de implementação do método e outros fatores que serão averiguados posteriormente. Além disso, como já foi observado na seção 3.2.1, com a presença de um maior número de elementos, a tendência é que sejam necessários menos graus de liberdade para a mesma aproximação $p$.

\subsubsection{Cisalhamento de uma Chapa}

O problema a seguir, encontra-se representado na Figura 3.8(a) e corresponde a uma chapa em estado plano de deformação sujeita às seguintes condições de contorno: 


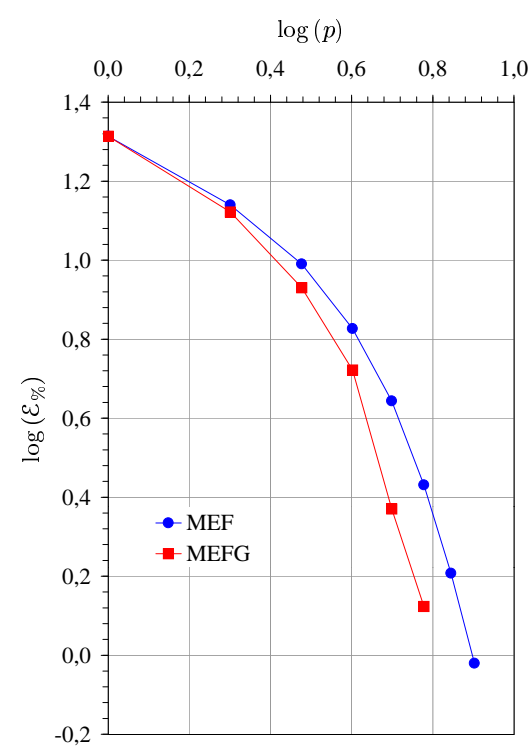

(a) Quanto a $p$

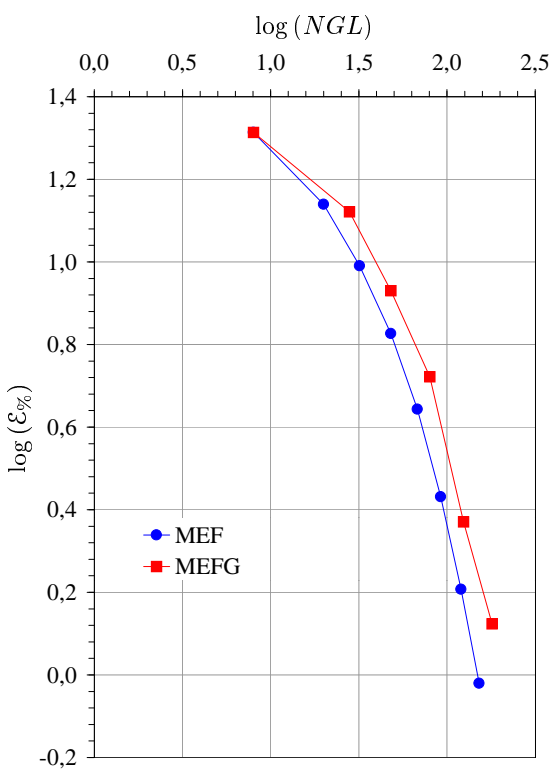

(b) Quanto ao NGL

Figura 3.7: Gráficos comparativos MEF x MEFG

- deslocamento vertical $u_{y}=0$ ao longo dos lados $F G$ e $E C$;

- deslocamento horizontal $u_{x}=-1 \mathrm{~mm}$ ao longo do lado $F G$;

- deslocamento horizontal $u_{x}=1 \mathrm{~mm}$ ao longo do lado $E C$;

A chapa encontra-se, portanto, solicitada por cisalhamento. Apenas o primeiro quadrante foi considerado e a discretização adotada está representada na Figura 3.8(b). Para o módulo de elasticidade adotou-se, $E=1 \mathrm{MPa}$ e o valor de $a=2 \mathrm{~m}$.

\begin{tabular}{|c|c|c|c|c|}
\hline \multirow{2}{*}{$\mathrm{p}$} & \multicolumn{2}{|c|}{$\nu=0,3$} & \multicolumn{2}{c|}{$\nu=0,4999$} \\
\cline { 2 - 5 } & $\mathcal{U}_{p}$ & $\mathcal{E}_{\%}$ & $\mathcal{U}_{p}$ & $\mathcal{E}_{\%}$ \\
\hline \hline 1 & 0,1411 & 28,23 & 0,1665 & 55,77 \\
\hline 2 & 0,1328 & 12,81 & 0,1421 & 34,49 \\
\hline 3 & 0,1325 & 11,73 & 0,1418 & 34,11 \\
\hline 4 & 0,1316 & 8,53 & 0,1323 & 20,40 \\
\hline 5 & 0,1312 & 6,40 & 0,1298 & 14,87 \\
\hline 6 & 0,1310 & 5,04 & 0,1288 & 11,84 \\
\hline
\end{tabular}

Tabela 3.8: Análises pelo MEFG enriquecido polinomialmente. Para $\nu=0,3000$ tem-se $\mathfrak{U}=$ $0,130680 N \times m m$ e para $\nu=0,4999, \mathcal{U}=0,127035 N \times m m$, DUARTE (1991).

Duas análises com enriquecimento polinomial $p$, da mesma forma que na seção 3.2.2, foram realizadas variando-se os valores do coeficiente de Poisson, $\nu=0,3$ 


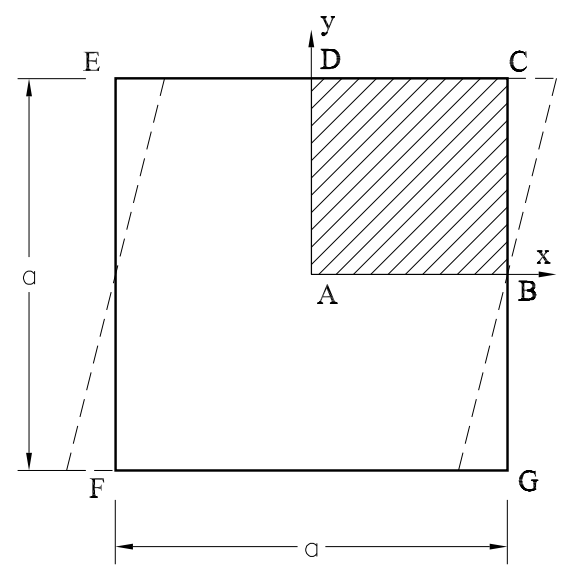

(a) Geometria

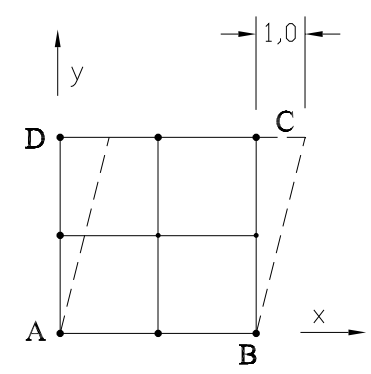

Figura 3.8: Estudo quanto ao travamento de Poisson

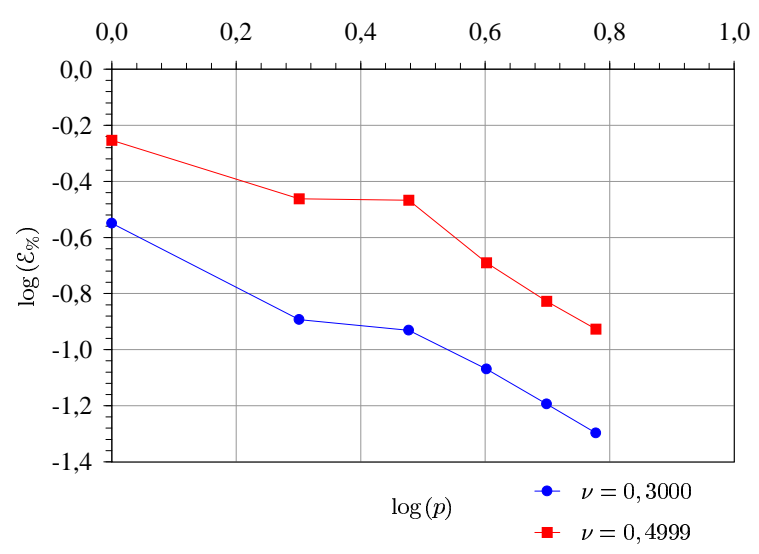

Figura 3.9: Análise quanto ao travamento de Poisson

e 0,499 9. Os resultados para estas análises, quanto à energia de deformação $\mathcal{U}_{p}$ encontram-se reunidos na Tabela $3.8^{5}$ e Figura 3.9. Para ambos os valores de $\nu$ não ocorreu alteração quanto à taxa de convergência, não havendo, portanto, interferência do travamento. Observou-se, contudo, uma diferença na posição das curvas, ou seja, para $\nu=0,4999$ os resultados estão sempre acima daqueles obtidos para um mesmo $p \operatorname{com} \nu=0,3000$, análoga à encontrada para o MEF hierárquico, como é verificado, por exemplo, em DUARTE (1991).

\footnotetext{
${ }^{5}$ Para esse problema, como a estrutura é submetida a deslocamentos impostos, à medida que sua aproximação é melhorada, tornando-se mais flexível, as tensões resultantes são reduzidas. Como conseqüência, ocorre uma diminuição da energia de deformação interna, mas o princípio da estacionaridade da energia potencial permanece válido.
} 


\subsubsection{Chapa em L}

Neste problema, procura-se mostrar como a estratégia de enriquecimento utilizada no MEFG pode simplificar bastante a análise de problemas com a presença de singularidades. Para isso, considera-se a chapa em forma de L da Figura 3.10(a), solicitada ao longo dos lados $A B, B C, E F$ e $F A$ pelo seguinte campo de tensões:

$$
\begin{gathered}
\left.\left.\sigma_{x}=A_{1} \lambda_{1} r^{\lambda_{1}-1}\left\{\left[2-Q_{1}\left(\lambda_{1}+1\right)\right] \cos \left(\lambda_{1}-1\right) \theta-\left(\lambda_{1}-1\right) \cos \left(\lambda_{1}-3\right) \theta\right\}(3.4)\right] \cos \left(\lambda_{1}-1\right) \theta+\left(\lambda_{1}-1\right) \cos \left(\lambda_{1}-3\right) \theta\right\} \\
\sigma_{y}=A_{1} \lambda_{1} r^{\lambda_{1}-1}\left\{\left[2+Q_{1}\left(\lambda_{1}+1\right)\right.\right. \\
\tau_{x y}=A_{1} \lambda_{1} r^{\lambda_{1}-1}\left\{\left(\lambda_{1}-1\right) \operatorname{sen}\left(\lambda_{1}-3\right) \theta+Q_{1}\left(\lambda_{1}+1\right) \operatorname{sen}\left(\lambda_{1}-1\right) \theta\right\}(3
\end{gathered}
$$

que produz deslocamentos nas direções $x$ e $y$ dados por:

$$
\begin{aligned}
& u_{x}=\frac{A_{1}}{2 G} r^{\lambda_{1}}\left\{\left[\kappa-Q_{1}\left(\lambda_{1}+1\right)\right] \cos \lambda_{1} \theta-\lambda_{1} \cos \left(\lambda_{1}-2\right) \theta\right\} \\
& u_{y}=\frac{A_{1}}{2 G} r^{\lambda_{1}}\left\{\left[\kappa+Q_{1}\left(\lambda_{1}+1\right)\right] \operatorname{sen} \lambda_{1} \theta+\lambda_{1} \operatorname{sen}\left(\lambda_{1}-2\right) \theta\right\}
\end{aligned}
$$

onde:

- $A_{1}$ é uma constante equivalente ao fator de intensidade de tensões no modo I em mecânica da fratura elástica;

- $\lambda_{1}=0,544483737$ e $Q_{1}=0,543075579$ são constantes determinadas para que as soluções (3.44) e (3.45) satisfaçam tanto o equilíbrio quanto as condições de tensão nula ao longo dos lados $C D$ e $D E$;

- considera-se a condição de estado plano de deformação e, por isso, $\kappa=3-4 \nu$;

Maiores detalhes sobre a obtenção das expressões para o campo de tensões de deslocamento podem ser encontradas, por exemplo, em SZABÓ; BABUŠKA (1991).

Um aspecto relevante da chapa em questão, e explicado por sua forma geométrica característica, é a elevada concentração de tensões na vizinhança do ponto $D$. O campo de tensões é, na verdade, singular, o que penaliza a taxa de convergência dos métodos que se utilizam do enriquecimento polinomial para aproximar a solução. Para ilustrar esse fato, uma seqüência de aproximações polinomiais, $\boldsymbol{u}_{p}$, são empregadas da mesma forma que na seção 3.2.2. A malha utilizada para definir a PU é descrita na Figura 3.10(b). Os resultados referentes à energia de deformação e ao erro relativo $\mathcal{E}_{\%}$, para cada nível de refinamento polinomial são apresentados na Tabela 3.9, sob a denominação de Seqüência I. Para confrontação, são também apresentados os resultados 


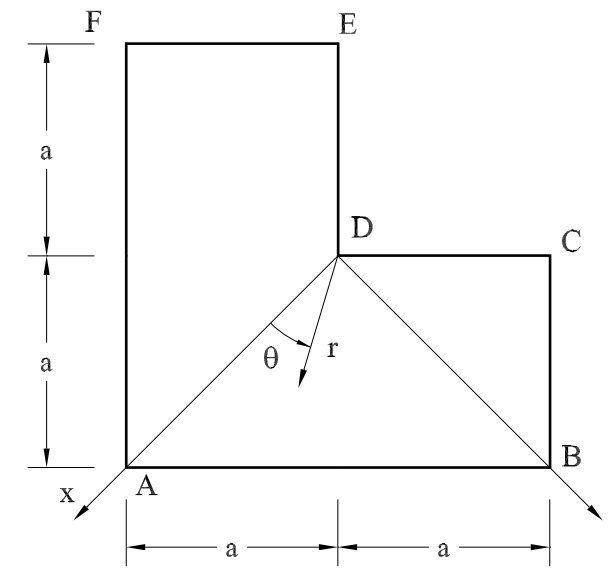

(a) Geometria

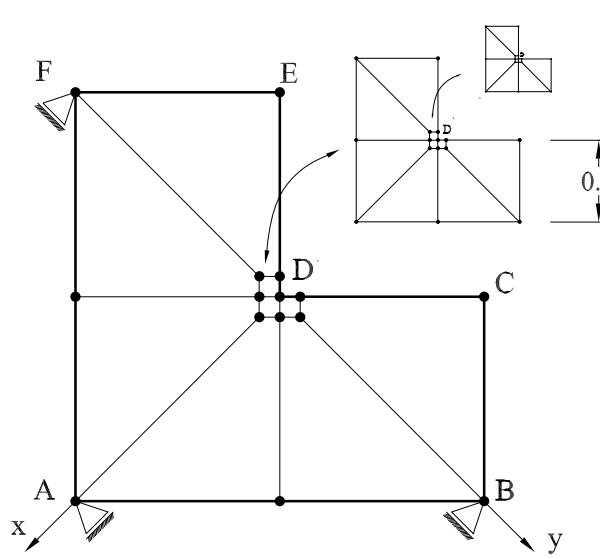

(c) Seqüência II

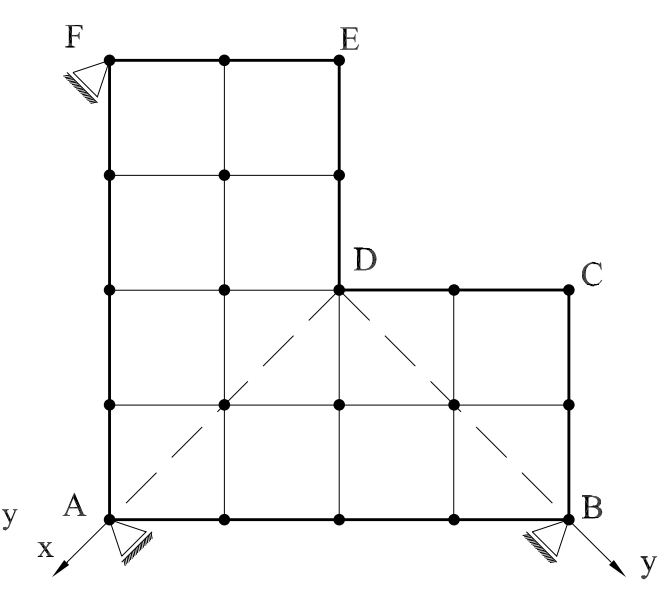

(b) Seqüência I

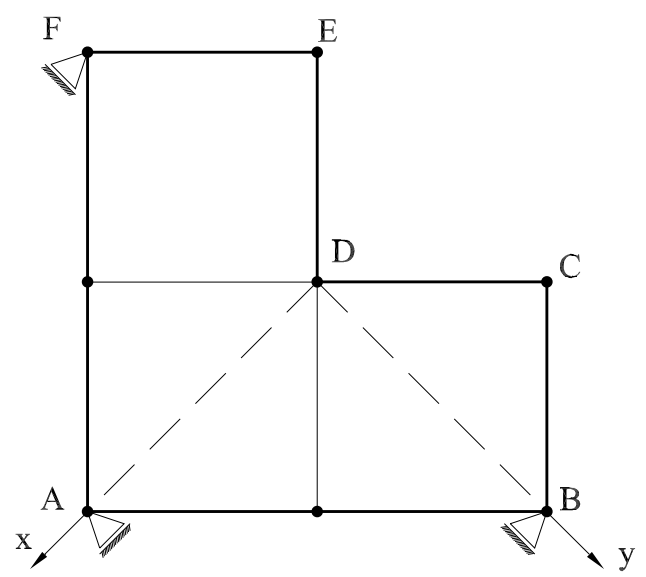

(d) Seqüência III

Figura 3.10: Chapa em L - geometria e malhas utilizadas nas seqüências de refinamento consideradas 
das análises de SZABÓ; BABUŠKA; CHAYAPATHY (1989) para o MEF hierárquico com a mesma seqüência de refinamento. No gráfico da Figura 3.11 exibe-se a curva que relaciona o erro $\mathcal{E}_{\%}$ com o NGL empregado. Observa-se uma convergência algébrica para as análises realizadas com os dois métodos. A convergência exponencial, típica dos refinamentos polinomiais, é portanto penalizada devido a singularidade no campo de tensões, SZABÓ; BABUŠKA (1991).

Para recuperar a taxa exponencial emprega-se uma seqüência de "malhas geométricas”, SZABÓ; BABUŠKA (1991), reunidas sob denominação de Seqüência II. O refinamento realizado é do tipo $h p$, em que, juntamente com o refinamento $h$ graduado conforme se mostra na Figura 3.10(c), é realizado um enriquecimento $p$ não-uniforme para o qual, com origem no ponto nodal $D$, o grau da aproximação aumenta linearmente a partir da unidade. Os resultados destas análises são também apresentados na Tabela 3.9 e gráfico da Figura 3.11, verificando-se sua independência com relação ao grau de singularidade da solução.

\begin{tabular}{|c|c|c|c|c|c|c|c|c|}
\hline \multicolumn{3}{|c|}{ MEF } & \multicolumn{6}{|c|}{ MEFG } \\
\hline \multirow[b]{2}{*}{ NGL } & \multicolumn{2}{|c|}{ Seqüência I } & \multirow[b]{2}{*}{ NGL } & \multicolumn{2}{|c|}{ Seqüência I } & \multirow[b]{2}{*}{ NGL } & \multicolumn{2}{|c|}{ Seqüência II } \\
\hline & $\mathcal{U}_{p}$ & $\varepsilon_{\%}$ & & $\mathcal{U}_{p}$ & $\varepsilon_{\%}$ & & $\mathcal{U}_{p}$ & $\mathcal{E}_{\%}$ \\
\hline 39 & 3,7702104 & 30,42 & 39 & 3,7702105 & 30,42 & 13 & 3,5024183 & 39,62 \\
\hline 103 & 3,9524576 & 22,06 & 123 & 3,9524575 & 22,06 & 41 & 3,7850141 & 29,82 \\
\hline 167 & 3,9751308 & 20,78 & 207 & 4,0214464 & 17,90 & 111 & 4,0988735 & 11,57 \\
\hline 255 & 4,0334329 & 17,07 & 333 & 4,0590699 & 15,16 & 223 & 4,1471773 & 4,21 \\
\hline 367 & 4,0718450 & 14,11 & 501 & 4,0913034 & 12,34 & 391 & 4,1532979 & 1,73 \\
\hline 503 & 4,0952969 & 11,94 & 711 & 4,1054160 & 10,87 & - & - & - \\
\hline 663 & 4,110 & 10,29 & 963 & 4,1171 & 9,49 & 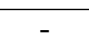 & - & - \\
\hline 847 & 4,1202816 & 9,08 & 1257 & 4,1250942 & 8,42 & - & - & - \\
\hline
\end{tabular}

Tabela 3.9: Resultados numéricos comparativos para a energia de deformação normalizada, $\left(U_{p} E / A_{1}^{2} a^{2 \lambda_{1}} t\right)$, entre o MEF, SZABÓ; BABUŠKA (1991) e o MEFG. Solução analítica exata de $U E / A_{1}^{2} a^{2 \lambda_{1}} t=4,15454423$ obtida de SZABÓ; BABUŠKA; CHAYAPATHY (1989)

Uma alternativa interessante de aplicação do MEFG refere-se à utilização das próprias soluções (3.44) e (3.45) para o enriquecimento da aproximação. Desse modo, em um nó $\boldsymbol{x}_{j}$ qualquer, a PU, pode ser enriquecida com os seguintes conjuntos de funções:

$$
\begin{aligned}
\mathfrak{J}_{j}^{x} & =\left\{1,1-\frac{u_{x}(\boldsymbol{x})}{u_{x}\left(\boldsymbol{x}_{j}\right)}, p_{1}, p_{2}, \cdots, p_{q_{j}(p)}\right\} \\
\mathfrak{J}_{j}^{y} & =\left\{1,1-\frac{u_{y}(\boldsymbol{x})}{u_{y}\left(\boldsymbol{x}_{j}\right)}, p_{1}, p_{2}, \cdots, p_{q_{j}(j)}\right\}
\end{aligned}
$$


Observe que o enriquecimento polinomial $p_{i}$ é utilizado juntamente com as novas funções $u_{x}$ e $u_{y}$. As aproximações nas direções $x$ e $y$ são, portanto, obtidas conforme a expressão (2.31):

$$
\begin{aligned}
& \tilde{u}_{x}(\boldsymbol{x})=\sum_{j=1}^{N} N_{j}(\boldsymbol{x})\left\{u_{j}^{x}+\sum_{i=1}^{q_{j}(p)} p_{i} b_{j i}^{x}+\left[1-\frac{u_{x}(\boldsymbol{x})}{u_{x}\left(\boldsymbol{x}_{j}\right)}\right] d_{j}^{x}\right\} \\
& \tilde{u}_{y}(\boldsymbol{x})=\sum_{j=1}^{N} N_{j}(\boldsymbol{x})\left\{u_{j}^{y}+\sum_{i=1}^{q_{j}(p)} p_{i} b_{j i}^{y}+\left[1-\frac{u_{y}(\boldsymbol{x})}{u_{y}\left(\boldsymbol{x}_{j}\right)}\right] d_{j}^{y}\right\}
\end{aligned}
$$

onde $d_{j}^{x}$ e $d_{j}^{y}$ são parâmetros associados às novas função não polinomiais introduzidas ao enriquecimento.

Nota-se que, no caso de se enriquecer diretamente com as funções (3.44) e (3.45), as aproximações em um ponto $\boldsymbol{x}_{j}$, por exemplo, seriam definidas como:

$$
\begin{aligned}
& \tilde{u}_{x}\left(\boldsymbol{x}_{j}\right)=u_{j}^{x}+u_{x}\left(\boldsymbol{x}_{j}\right) d_{j}^{x} \\
& \tilde{u}_{y}\left(\boldsymbol{x}_{j}\right)=u_{j}^{y}+u_{y}\left(\boldsymbol{x}_{j}\right) d_{j}^{y}
\end{aligned}
$$

os parâmetros $u_{j}^{x}$ e $u_{j}^{y}$ perderiam o significado físico de deslocamentos nodais e, por isso, não haveria como se impor diretamente as condições de contorno essenciais no ponto nodal $\boldsymbol{x}_{l}$. Para evitar a aplicação do Método dos Multiplicadores de Lagrange, como na seção 2.3 para os métodos sem malha, optou-se por modificar as funções (3.44) e (3.45), introduzindo-as da maneira descrita em (3.48) e (3.49). Dessa maneira, os parâmetros nodais $u_{j}^{x}$ e $u_{j}^{y}$ voltam a corresponder diretamente aos deslocamentos, permitindo com que as condições de contorno sejam impostas diretamente. Este artifício em nada prejudica a qualidade da solução como poderá ser mostrado através dos resultados numéricos apresentados a seguir.

Duas seqüências de aproximações, variando-se o enriquecimento polinomial $p$, foram utilizadas para ilustrar a estratégia acima descrita. Em todas elas a PU é definida sobre a malha da Figura 3.10(d). Na primeira delas, referenciada como IIIa, apenas o nó $D$ é enriquecido com o conjunto de funções (3.46) e (3.47), enquanto que na segunda, denominada Seqüência IIIb, todos os nós são enriquecidos. Os resultados de tais análises encontram-se reunidos na Tabela 3.10 e gráfico da Figura 3.11.

A partir dos resultados apresentados, observa-se que as taxas de convergências obtidas com os enriquecimentos especiais, (3.46) e (3.47), superam os resultados das malhas geométricas. Para o caso da Sequiência IIIb a solução é praticamente a exata 


\begin{tabular}{|c|c|c|c|c|c|}
\hline \multicolumn{5}{|c|}{ MEFG } \\
\hline \multirow{2}{*}{ NGL } & \multicolumn{2}{|c|}{ Seqüência IIIa } & & \multicolumn{2}{|c|}{ Seqüência IIIb } \\
\cline { 2 - 3 } \cline { 5 - 6 } & $\mathcal{U}$ & $\mathcal{E}_{\%}$ & NGL & $\mathcal{U}$ & $\mathcal{E}_{\%}$ \\
\hline \hline 14 & 3,9341859 & 23,03 & 21 & 4,1545434 & 0,04 \\
\hline 46 & 4,1328609 & 7,22 & 61 & 4,1545434 & 0,04 \\
\hline 78 & 4,1466355 & 4,36 & 93 & 4,1545434 & 0,04 \\
\hline 126 & 4,1522908 & 2,33 & 133 & 4,1545434 & 0,04 \\
\hline 190 & 4,1534645 & 1,61 & 197 & 4,1545434 & 0,04 \\
\hline
\end{tabular}

Tabela 3.10: Resultados para a energia de deformação, $\left(\tilde{U} E / A_{1}^{2} a^{2 \lambda_{1}} t\right)$, através do MEFG. Solução analítica exata, SZABÓ; BABUŠKA; CHAYAPATHY (1989) $\mathcal{U E} / A_{1}^{2} a^{2 \lambda_{1}} t=4,15454423$

desde o início do refinamento $p$. Este fato já era esperado, pois a própria solução exata está sendo empregada em todo o domínio, e não apenas para eliminar o efeito da singularidade. O pequeno erro encontrado para esse caso é proveniente da imprecisão na integração numérica comentada no próximo parágrafo.

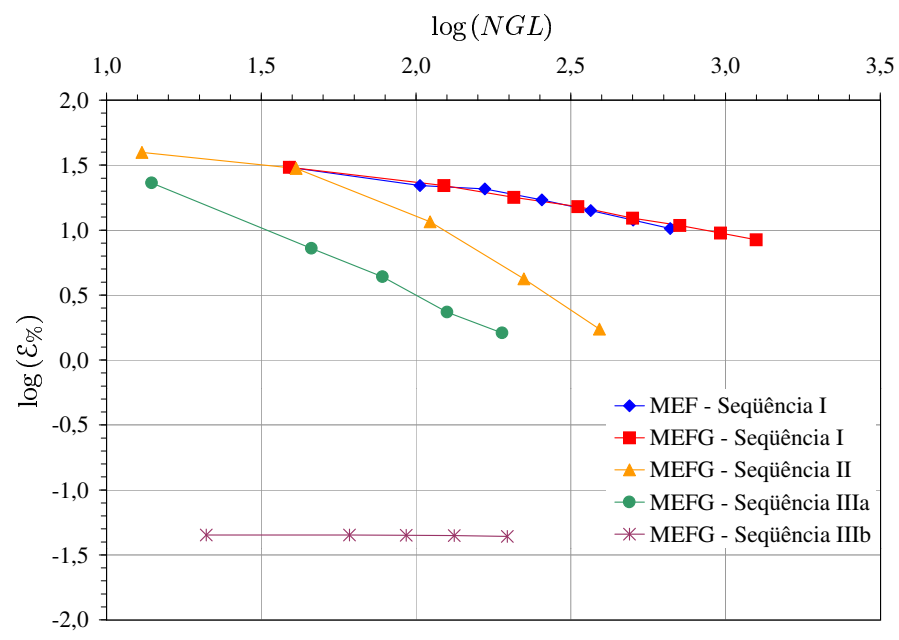

Figura 3.11: Análises para diversos tipos de refinamento 
A integração numérica torna-se um ponto importante para os enriquecimentos com funções não-polinomiais realizados para problemas desse tipo. Quando são utilizadas funções com elevados gradientes, a quadratura de Gauss torna-se insuficiente para se aproximar exatamente a integral procurada. Procedimentos adaptativos de integração numérica devem, então, ser empregados, como é observado, por exemplo, em DUARTE; BABUŠKA; ODEN (2000). Em particular, no trabalho de STROUBOULIS; BABUŠKA; COPPS (2000), sugere-se o algoritmo DECUHR introduzido em ESPELID (1994), baseado em sub-divisões não-uniformes do elemento finito, na direção do ponto em que se encontra a singularidade. Para o presente trabalho, nenhum desses procedimentos foram implementados. Dessa forma, uma elevada quantidade de pontos de Gauss-Legendre teve que ser utilizada para cada um dos três elementos das malhas das Seqüências IIIa e IIIb $(N G=50 \times 50)$.

\subsubsection{Considerações Complementares}

A possibilidade de conferir à aproximação o comportamento de uma função singular abre caminho para estudos no campo da Mecânica da Fratura Elástica.

Recentemente, o grupo de Belytshcko, desenvolvedor do MGLE, re-direcionou os estudos para o enriquecimento da PU, obtida pelo MEF, com funções que simulam o campo de tensões ao redor da trinca. Em BELYTSCHKO; BLACK (1999) e MOËS; DOLBOW; BELYTSCHKO (1999) denomina-se essa estratégia de Método dos Elementos Finitos Extendido, MEFX (Extended Finite Element Method - XFEM). Tal abordagem é, contudo, equivalente à proposta em MELENK; BABUŠKA (1996) e ODEN; DUARTE; ZIENKIEWICZ (1998) e que, mais tarde, originou o MEFG. Como contribuição, podem ser destacadas as estratégias criadas para se considerar a propagação da trinca, DOLBOW (1999), MOËS; DOLBOW; BELYTSCHKO (1999) e STOLARSKA (2001).

Em STROUBOULIS; BABUŠKA; COPPS (2000), por sua vez, referencia-se diretamente o MEFG, e uma abordagem bastante genérica é apresentada para introdução de vazios e descontinuidades no meio, sem que a malha de elementos seja alterada. Já em DUARTE (2001), o MEFG é empregado para simular a propagação dinâmica de trinca em um meio sob representação tri-dimensional. Funções especiais, a exemplo do que foi utilizado na seção 3.2.4, são adotadas para aproximar o campo de tensões ao redor da trinca. Para que a propagação da fratura possa ser simulada sem alterações na malha de elementos, funções de Shepard são combinadas com as funções Lagrangianas 
na construção da PU.

Estratégias para a aplicação do MEFG em problemas da Mecânica da Fratura Elástica já se encontram, portanto, em franco desenvolvimento. Um caminho ainda não explorado corresponde ao estudo da propagação de fratura em meios com dano. Nesse contexto, ocorre um abatimento da concentração de tensões na extremidade da trinca devido à formação de uma zona de processo. Outro ponto de interesse consiste na simulação da transição entre a formação e distribuição de micro-fissuras e o surgimento da trinca macroscópica. Através do MEFG, torna-se bastante simples modificar a descrição da solução durante a análise, pois o enriquecimento não introduz nenhum nó adicional. Dessa forma, a análise pode ser realizada com o enriquecimento polinomial para a fase em que o dano é o processo dominante e, no instante de transição para a fratura, novas funções podem ser introduzidas. A ferramenta numérica talhada para esse tipo de aplicação existe, portanto. Resta, contudo, que seja adaptada para tal. Neste trabalho, procura-se estabelecer as bases para tal desenvolvimento. Antes, porém, é necessário que alguns conceitos da Mecânica do Dano Contínuo sejam introduzidos. 


\section{Capítulo 4}

\section{Modelo Constitutivo}

A teoria do dano tem como domínio de estudo os fenômenos de deterioração incidentes sobre o material, compreendidos entre o que seria um estado virgem, sem a presença de imperfeições, e a formação das primeiras trincas macroscópicas. Tais fenômenos abrangem o processo de "nucleação" e crescimento de cavidades e de trincas microscópicas, distribuídas ao longo do volume e da superfície do material. A Mecânica do Dano Contínuo procura descrever o processo de danificação do material real a partir do comportamento mecânico macroscópico de um meio equivalente considerado contínuo. Para isso, introduzem-se variáveis para quantificar a deterioração desse meio e que penalizam suas propriedades elásticas. Detalhes sobre a Mecânica do Dano Contínuo, fundamentada hoje na termodinâmica dos processos irreversíveis, podem ser encontrados em KRAJCINOVIC; LEMAITRE (1987), LEMAITRE; CHABOCHE (1990) e LEMAITRE (1992).

Existem atualmente, dentro da Mecânica do Dano Contínuo, diversos modelos matemáticos que se aplicam à simulação do comportamento físico não-linear de estruturas em concreto. Nas próximas seções, após discorrer sobre alguns conceitos fundamentais, são apresentados dois desses modelos constitutivos conhecidos como Modelo de Mazars e Modelo de La Borderie ${ }^{1}$. Tais modelos foram utilizados neste trabalho devido à sua simplicidade e aplicabilidade aos tipos de problemas estudados. Para o caso particular das análises realizadas relativamente a estados planos de tensão e de deformação, apenas o Modelo de Mazars, em sua formulação não-local, foi empregado. Alguns detalhes de tal formulação, bem como a razão para seu emprego são abordados ao final deste capítulo.

\footnotetext{
${ }^{1}$ Ambos os modelos são conhecidos pelo nome dos pesquisadores que os propuseram, MAZARS (1984) e LA BORDERIE (1991)
} 


\subsection{Conceitos da Mecânica do Dano Contínuo}

Não é o objetivo deste trabalho aprofundar-se nos fundamentos da Mecânica do Dano Contínuo, mas alguns de seus conceitos merecem ser registrados para uma melhor compreensão dos modelos constitutivos adotados para as análises numéricas. São eles:

- elemento de volume representativo;

- tensão efetiva;

- princípio da deformação equivalente.

O elemento de volume representativo é definido como uma região do material grande o suficiente para representar a média dos micro-processos presentes e não tão pequena para que, assim, as equações da Mecânica do Contínuo mantenham seu significado, LEMAITRE (1992), Figura 4.1.

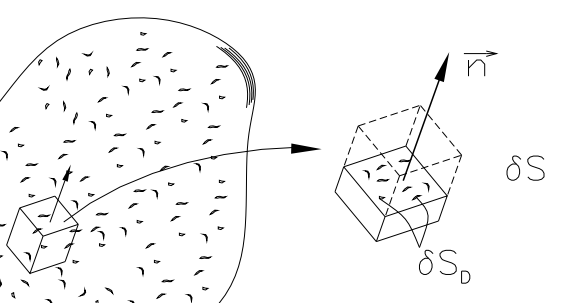

Figura 4.1: Elemento de volume representativo

Tomando-se um ponto no interior do volume representativo, o estado de deterioração, segundo uma dada direção definida pelo versor $\overrightarrow{\boldsymbol{n}}$, é quantificado pela variável $D_{n}$, através da razão:

$$
D_{n}=\frac{\delta S_{D}}{\delta S}
$$

onde $\delta S$ é a área da interseção entre o elemento e o plano normal a $\overrightarrow{\boldsymbol{n}}$ no ponto. Já $\delta S_{D}$ representa a parcela de $\delta S$ que corresponde aos vazios ou descontinuidades geométricas. Dessa maneira, definem-se:

- $D_{n}=0 \Rightarrow$ elemento de volume íntegro;

- $D_{n}=1 \Rightarrow$ elemento de volume totalmente danificado. 
Considera-se, agora, que o elemento de volume esteja solicitado na direção $\vec{n}$ por uma força $F$, de tal maneira que nesta direção tenha-se a tensão nominal dada por $\sigma=F / \delta S$. Se no lugar de $\delta S$ for usada a área que realmente resiste à solicitação, $\delta S-\delta S_{D}$, obtém-se a tensão efetiva presente em um ponto da parte íntegra de $\delta S$ :

$$
\sigma_{\text {ef }}=\frac{F}{\delta S-\delta S_{D}}
$$

O princípio da deformação equivalente estabelece que o estado de deformação em qualquer ponto do meio danificado, segundo a direção $\vec{n}$, possa ser obtido pela deformação elástica da parte íntegra, LEMAITRE; CHABOCHE (1990). Isto significa que, para o elemento de volume representativo descrito acima, é possível calcular a deformação $\varepsilon$ como:

$$
\varepsilon=\frac{\sigma_{\mathrm{ef}}}{E_{0}}=\frac{\sigma}{E_{0}\left(1-D_{n}\right)}
$$

sendo $E_{0}$ o módulo de elasticidade da parte íntegra.

Nota-se que o princípio da deformação equivalente permite com que a tensão nominal $(\sigma)$ possa ser empregada para qualquer estado de deterioração, bastando que se penalize o módulo de elasticidade pela grandeza $1-D_{n}$. No caso geral de solicitação multi-axial, o dano e sua lei de evolução adquirem direções preferenciais segundo a orientação de $\overrightarrow{\boldsymbol{n}}$. Dentro de certos limites, porém, pode-se adotar a hipótese de homogeneidade do processo de danificação, o que torna o problema muito simples uma vez que em todas as direções a medida da danificação será a mesma, ou seja: $D_{n}=D, \forall \overrightarrow{\boldsymbol{n}}$.

\subsection{Modelo de Mazars}

Comparado aos demais modelos empregados para o concreto, o modelo de Mazars apresenta como vantagem sua simplicidade e um número relativamente pequeno de parâmetros a identificar. O estado de danificação local é representado pela variável escalar de dano $D$, o que é suficiente para representar, de modo satisfatório, o comportamento do concreto sob solicitações especiais. As hipóteses básicas do modelo são as seguintes:

- o concreto em processo de dano evolutivo é considerado como um meio elástico sem deformações residuais, Figura 4.2(b), diferentemente do que se obtém experimentalmente, Figura 4.2(a); 
- apenas as extensões, nas direções principais de deformação, contribuem para a formação e evolução real do dano;

- o carregamento é proporcionalmente crescente (radial);

- o dano preserva a isotropia inicial do meio;

- o processo de danificação inicia-se localmente quando a grandeza denominada deformação equivalente, e representada por $\varepsilon_{e q}$, for superior a um certo valor de referência $\varepsilon_{d 0}$.

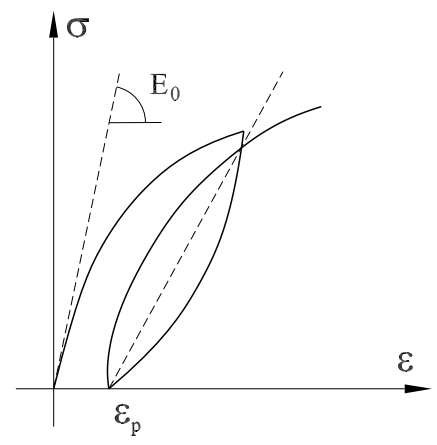

(a) Comportamento real

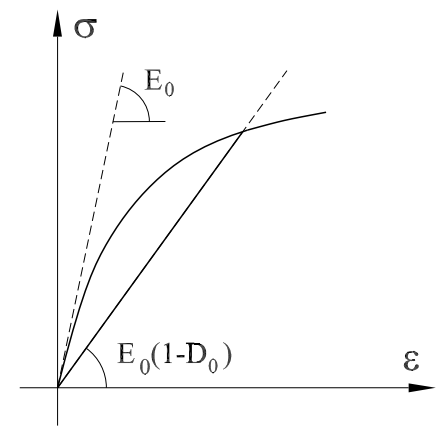

(b) Modelo de Mazars

Figura 4.2: Relação constitutiva

O dano $D$ penaliza diretamente a rigidez elástica do material e a relação constitutiva do modelo é expressa através da hipótese de deformações equivalentes. Resumindo, o modelo de Mazars leva à seguinte relação secante para o caso de carregamento proporcional:

$$
\boldsymbol{\sigma}=(1-D) \boldsymbol{C}_{0} \boldsymbol{\varepsilon}
$$

em que $\boldsymbol{C}_{0}$ corresponde à matriz das propriedades elásticas do material íntegro, que estabelece a relação constitutiva entre os campos de tensão e deformação em sua forma compacta de representação vetorial.

Segundo a abordagem de PEREGO (1989), a variável $D$ é definida através da combinação linear:

$$
D=\alpha_{T} D_{T}+\alpha_{C} D_{C}
$$


em que $D_{T}$ e $D_{C}$ representam o dano associado a estados uni-axiais de tração e compressão respectivamente, dados por:

$$
D_{T}=1-\frac{\varepsilon_{d 0}\left(1-A_{T}\right)}{\varepsilon_{e q}}-\frac{\left(1-A_{T}\right)}{e^{B_{T}\left(\varepsilon_{e q}-\varepsilon_{d 0}\right)}} ; \quad D_{C}=1-\frac{\varepsilon_{d 0}\left(1-A_{C}\right)}{\varepsilon_{e q}}-\frac{\left(1-A_{C}\right)}{e^{B_{C}\left(\varepsilon_{e q}-\varepsilon_{d 0}\right)}}
$$

onde:

- $A_{T}, B_{T}, A_{C}$ e $B_{C}$ são parâmetros característicos do material e que podem ser identificados em ensaios uni-axiais, ÁLVARES (1993);

- $\alpha_{T}$ e $\alpha_{C}$ são coeficientes obtidos a partir do estado de deformação em cada ponto do corpo, tendo-se sempre $\alpha_{T}+\alpha_{C}=1$. Naturalmente, para a tração pura $\alpha_{T}=1$ e para a compressão pura $\alpha_{C}=1$;

- $\varepsilon_{e q}=\sqrt{\left\langle\varepsilon_{1}\right\rangle_{+}^{2}+\left\langle\varepsilon_{2}\right\rangle_{+}^{2}+\left\langle\varepsilon_{3}\right\rangle_{+}^{2}}$ é denominada deformação equivalente obtida em função da parte positiva das componentes de deformações principais. Sendo $\langle\cdot\rangle_{+}=1 / 2(\cdot+|\cdot|)$

- $\varepsilon_{d 0}$ é um valor de referência para a deformação equivalente, característico do material, acima do qual ocorre o início da danificação. Por analogia a esse parâmetro, define-se $\varepsilon_{d}$, variável que governa a evolução do dano. Em cada ponto do material, tem-se, inicialmente, $\varepsilon_{d}=\varepsilon_{d 0}$. À medida que a deformação equivalente ultrapassa esse valor em algum ponto, a variável $\varepsilon_{d}$ passa a ser atualizada com tal deformação.

Em sua formulação em taxas, a relação constitutiva passa a ser expressa por:

$$
\dot{\boldsymbol{\sigma}}=(1-D) \boldsymbol{C}_{0} \dot{\varepsilon}-\dot{D} \boldsymbol{C}_{0} \varepsilon
$$

A taxa de dano expressa em função da taxa de deformação envolve, entretanto, uma expressão complexa, gerando uma matriz de rigidez não-simétrica para uso na aproximação de Galerkin do PVC, apêndice A. Por essa razão, e já tendo-se em vista o procedimento incremental-iterativo de Newton-Raphson, seção 5.2.1, para a solução do PVC, utiliza-se a formulação secante dada por:

$$
\Delta \boldsymbol{\sigma}=(1-D) \boldsymbol{C}_{0} \Delta \varepsilon
$$

ou seja:

$$
\Delta \sigma=C \Delta \varepsilon
$$


sendo:

$$
\boldsymbol{C}=(1-D) \boldsymbol{C}_{0}
$$

\subsection{Modelo de La Borderie}

Estruturas cujo o material é o concreto fissuram quando tracionadas. Apresentam, porém, a propriedade de recuperar a rigidez, caso o carregamento presente seja invertido, COMI (2000). Este aspecto, bastante peculiar, é denominado "efeito unilateral" e pode ser explicado, conforme descrito em seguida, à partir do mecanismo de deterioração do meio. Sob solicitação, surgem, no concreto, micro-fissuras perpendicularmente às direções de alongamento, produzindo, como resposta macroscópica, uma perda de rigidez da estrutura. Com a inversão do sinal da solicitação, tais fissuras tendem a se fechar e, como conseqüência, ocorre o fenômeno de enrijecimento. No modelo de Mazars, tal fenômeno não é considerado e, por isso, sua aplicação não é indicada nos casos de solicitações de carregamento cíclico.

Em LA BORDERIE (1991) é sugerido um modelo adequado à simulação do “efeito unilateral", através da introdução de duas variáveis, $D_{1}$ e $D_{2}$, relacionadas aos danos de tração e compressão respectivamente. Diferentemente do modelo de Mazars, estas variáveis não são combinadas, e seu processo de evolução é definido através do controle sobre o sinal das tensões principais. Descreve-se assim, com sucesso, o fenômeno do fechamento e abertura das fissuras simulando-se o "efeito unilateral" do concreto.

A apresentação do modelo é feita através da abordagem do método do estado local, descrito em LEMAITRE; CHABOCHE (1990), partindo-se do seguinte potencial de estado dado pela energia livre de Gibbs $\chi$ :

$$
\begin{aligned}
\chi= & \frac{\left(\langle\boldsymbol{\Sigma}\rangle_{+} \cdot\langle\boldsymbol{\Sigma}\rangle_{+}\right)}{2 E\left(1-D_{1}\right)}+\frac{\left(\langle\boldsymbol{\Sigma}\rangle_{-} \cdot\langle\boldsymbol{\Sigma}\rangle_{-}\right)}{2 E\left(1-D_{2}\right)}+\frac{\nu}{E}\left[(\boldsymbol{\Sigma} \cdot \boldsymbol{\Sigma})-\operatorname{Tr}^{2}(\boldsymbol{\Sigma})\right]+ \\
& \frac{\beta_{1} D_{1}}{E\left(1-D_{1}\right)} f_{d}(\operatorname{Tr}(\boldsymbol{\Sigma}))+\frac{\beta_{2} D_{2}}{E\left(1-D_{2}\right)} \operatorname{Tr}(\boldsymbol{\Sigma})+G_{1}\left(z_{1}\right)+G_{2}\left(z_{2}\right)
\end{aligned}
$$

em que $\nu$ é o coeficiente de Poisson, $\langle\boldsymbol{\Sigma}\rangle_{+}$e $\langle\boldsymbol{\Sigma}\rangle_{-}$representam as partes positiva e negativa do tensor de tensões $\Sigma, \operatorname{Tr}(\Sigma)=\Sigma_{i i}, \beta_{1}$ e $\beta_{2}$ são parâmetros do material relacionados à formação de deformações residuais, $G_{1}\left(z_{1}\right)$ e $G_{2}\left(z_{2}\right)$ são funções de encruamento de $z_{1}$ e $z_{2}$ (medidas de dano acumulado) e $f_{d}$ é uma função que assume 
valores de acordo com as condições de abertura e fechamento das micro-fissuras, sendo definida por:

$$
f_{d}(\operatorname{Tr}(\boldsymbol{\Sigma})) \stackrel{\text { def }}{=}\left\{\begin{array}{ccc}
\operatorname{Tr}(\boldsymbol{\Sigma}) & \text { quando } & \operatorname{Tr}(\boldsymbol{\Sigma}) \in(0,+\infty] \\
{\left[1+\frac{\operatorname{Tr}(\boldsymbol{\Sigma})}{2 \sigma_{f}}\right] \operatorname{Tr}(\boldsymbol{\Sigma})} & \text { quando } & \operatorname{Tr}(\boldsymbol{\Sigma}) \in\left(-\sigma_{f}, 0\right] \\
-\frac{\sigma_{f}}{2} \operatorname{Tr}(\boldsymbol{\Sigma}) & \text { quando } & \operatorname{Tr}(\boldsymbol{\Sigma}) \in\left(-\infty,-\sigma_{f}\right]
\end{array}\right.
$$

sendo $\sigma_{f}$ a tensão de fechamento das micro-fissuras.

O tensor de deformações $\boldsymbol{E}$ pode ser obtido derivando-se o potencial (4.10) com relação as tensões $\Sigma$ :

$$
\boldsymbol{E}=\frac{\partial \chi}{\partial \boldsymbol{\Sigma}}
$$

Em problemas bi-dimensionais de estado plano, por questão de simplicidade, os tensores $\boldsymbol{\Sigma}$ e $\boldsymbol{E}$ podem ser substituídos pelas formas vetoriais compactas $\boldsymbol{\sigma}$ e $\boldsymbol{\varepsilon}$. Adotando-se tal representação em (4.10), o vetor de deformações totais $\varepsilon$ pode ser obtido de maneira idêntica a (4.12):

$$
\varepsilon=\frac{\partial \chi}{\partial \boldsymbol{\sigma}}=\varepsilon^{e}+\varepsilon^{a}
$$

Observa-se que o campo de deformações é dividido em duas parcelas, uma elástica $\varepsilon^{e}$ e outra anelástica $\varepsilon^{a}$ :

$$
\begin{gathered}
\boldsymbol{\varepsilon}^{e}=\frac{\langle\boldsymbol{\sigma}\rangle_{+}}{E\left(1-D_{1}\right)}+\frac{\langle\boldsymbol{\sigma}\rangle_{-}}{E\left(1-D_{2}\right)}+\frac{\nu}{E}[\boldsymbol{\sigma}-\operatorname{Tr}(\boldsymbol{\sigma})] \boldsymbol{I} \\
\boldsymbol{\varepsilon}^{a}=\frac{\beta_{1} D_{1}}{E\left(1-D_{1}\right)} \frac{\partial f(\operatorname{Tr}(\boldsymbol{\sigma}))}{\partial \boldsymbol{\sigma}}+\frac{\beta_{2} D_{2}}{E\left(1-D_{2}\right)} \boldsymbol{I}
\end{gathered}
$$

permitindo-se que, diferentemente do modelo de Mazars, deformações residuais decorrentes do dano sejam consideradas.

As variáveis associadas ao dano são dadas por:

$$
\begin{aligned}
& Y_{1}=\frac{\partial \chi}{\partial D_{1}} \\
& Y_{2}=\frac{\partial \chi}{\partial D_{2}}
\end{aligned}
$$


$\mathrm{O}$ critério de dano pode ser expresso pelas leis $F_{\alpha}=Y_{\alpha}-Z_{\alpha}, \alpha=1,2$, onde $Z_{\alpha}$ são as variáveis termodinâmicas associadas às variáveis $z_{\alpha}$ e obtidas da seguinte forma:

$$
Z_{\alpha}=\frac{\partial G_{\alpha}\left(z_{\alpha}\right)}{\partial z_{\alpha}}=\left[Y_{o \alpha} \frac{1}{A_{\alpha}}\left(\frac{D_{\alpha}}{1-D_{\alpha}}\right)^{1 / B_{\alpha}}\right] \quad(\alpha=1,2)
$$

sendo $A_{\alpha}, B_{\alpha}$ e $Y_{o \alpha}$ parâmetros do material a serem identificados. Considerando-se, então, o critério $F_{\alpha}$, a evolução do dano pode ser caracterizada como:

Se $Y_{\alpha}<Z_{\alpha} \Rightarrow \dot{D}_{\alpha}=0$ resposta elástica-linear

Se $Y_{\alpha}=Z_{\alpha}$ e $\dot{Y}_{\alpha}>0 \Rightarrow \dot{D}_{\alpha} \neq 0$ e $D_{\alpha}=1-\frac{1}{1+\left[A_{\alpha}\left(Y_{\alpha}-Y_{o \alpha}\right)\right]^{B_{\alpha}}}$

Nas análise não-lineares, emprega-se para a solução do problema a relação constitutiva em sua forma incremental expressa por:

$$
\Delta \boldsymbol{\sigma}=\left(1-D_{\alpha}\right) \boldsymbol{C}_{0} \Delta \varepsilon
$$

onde $D_{\alpha}$ é substituído por $D_{1}$ ou $D_{2}$, de acordo com campo de tensões atuante. Maiores detalhes a esse respeito podem ser encontrados em LA BORDERIE (1991), ou mesmo em PAULA (2001) para o caso da implementação do modelo para problemas uni-dimensionais.

\subsection{Abordagem Não-Local}

O fenômeno de deterioração do meio que se busca reproduzir pela Mecânica do Dano Contínuo é, por hipótese, difuso. Esta abordagem contrapõe-se à Mecânica da Fratura em que se considera uma falha local ou trinca macroscópica. Existe, portanto, um limite para o campo de aplicação dos modelos de dano, devendo-se observar o instante em que o fenômeno de ruptura deixa de ser associado apenas à danificação evolutiva, transformando-se em propagação de uma fratura. A teoria da localização de deformações, discutida em OLIVER (1995) e MANZOLI; OLIVER; CERVERA (1998) por exemplo, permite estabelecer uma ligação entre estas duas abordagens. Alguma discussão a esse respeito é realizada no capítulo 7, no qual se vislumbra, por meio de uma análise numérica, um interessante caminho a ser trilhado. A atenção 
no momento, contudo, restringe-se unicamente à aplicação dos modelos de dano e é dentro dessa perspectiva que a análise do tipo não-local necessita ser introduzida.

Em COMI (2000), salienta-se que a presença do fenômeno de amolecimento (softening), induzido pelos modelos de dano, faz com que o problema de valor de contorno torne-se mal-posto, introduzindo perda de objetividade nos resultados numéricos. Uma das conseqüências é a dependência da resposta numérica à malha de elementos, DAVENNE; SAOURIDIS; PIAU (1989), o que, nos métodos sem malha, corresponderia a uma dependência ao suporte das funções aproximadoras. Como resultado, quanto menor o suporte dessas funções, menor a região em que o dano tende a ficar confinado, antecipando a localização de deformações.

A abordagem não-local é uma técnica de regularização que limita a zona de localização de deformações, fixando uma largura mínima para a concentração do dano. Consegue-se, assim, dependendo da largura adotada, uma melhor distribuição para o dano, necessária para que os modelos de dano possam ser aplicados sem perda de objetividade. Com os exemplos das Figuras 4.3(a) e 4.3(b) procura-se ilustrar a diferença entre análises realizadas através das abordagens local e não-local respectivamente. $\mathrm{Na}$ Figura 4.3(a) ocorre, para um determinado nível de força, a concentração do dano, porque o modelo tende a reproduzir a descontinuidade, representada na curva de resposta estrutural, decorrente da formação de uma fissura e a conseqüente perda momentânea da capacidade de carga. Realizando-se a mesma análise, em uma abordagem não-local, obtém-se uma resposta global equivalente, mas através de um distribuição difusa para o dano.

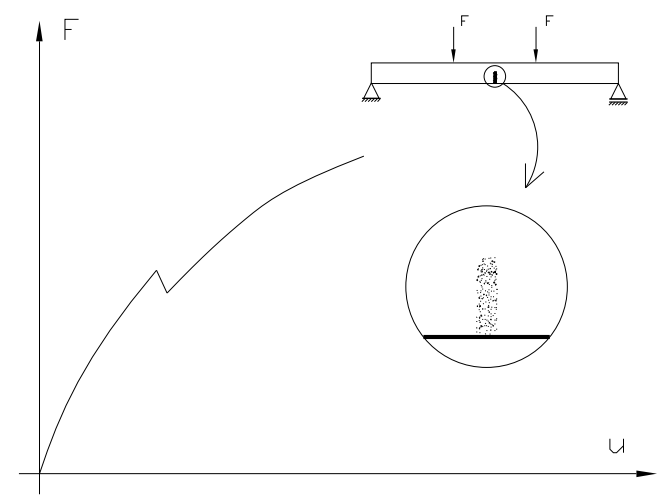

(a) Análise local

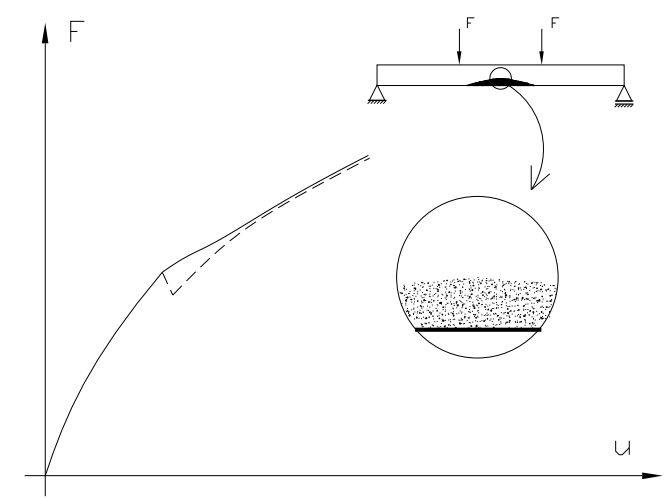

(b) Análise não-local

Figura 4.3: Representação das diferenças entre análise local e não-local. Curvas de força $F$ e deslocamento no meio do vão $u$, com a respectiva distribuição de dano 
Um aspecto significante em uma análise não-local é a definição da largura mínima para a concentração do dano. Este parâmetro está relacionado a um caráter não-local da deformação devido à heterogeneidade do meio e responsável pela maneira com que as micro-fissuras se distribuem. Em BAZǍNT; PIJAUDIER-CABOT (1989), o parâmetro $l_{c}$, denominado comprimento característico do material, é introduzido como grandeza representativa dessa heterogeneidade. Em outras palavras, $l_{c}$ define o volume representativo do material e, segundo MAZARS; PIJAUDIER-CABOT (1994), é da ordem de $3 d_{a}$, sendo $d_{a}$ o "tamanho" do maior agregado no concreto.

Uma maneira de se introduzir o caráter não-local, consiste em se empregar, localmente, uma média ponderada das variáveis de dano ou do campo de deformações nas vizinhanças de um ponto. Adota-se neste trabalho, a média das deformações, conforme estratégia apresentada em DAVENNE; SAOURIDIS; PIAU (1989) para o modelo de Mazars.

Assim sendo, como a evolução do dano depende da variável $\varepsilon_{e q}$, essa passa a ser calculada não mais localmente mas como uma média ponderada, $\bar{\varepsilon}_{e q}$, dos valores que assume em uma vizinhança, $V\left(r_{n l}\right)$ de raio $r_{n l} \approx 0,5 l_{c}$, correspondente ao elemento de volume representativo do material. Fisicamente, tal abordagem mostra-se mais coerente, uma vez que a danificação local é governada pelos mecanismos presentes em uma determinada região representativa do meio.

Dessa forma, tem-se:

$$
\bar{\varepsilon}_{e q}\left(\boldsymbol{x}_{i}\right)=\frac{\int_{V\left(r_{n l}\right)} g\left(\boldsymbol{x}-\boldsymbol{x}_{i}\right) \varepsilon_{e q}(\boldsymbol{x}) d V}{\int_{V\left(r_{n l}\right)} g\left(\boldsymbol{x}-\boldsymbol{x}_{i}\right) d V}
$$

sendo $g(\boldsymbol{x})$ uma função de ponderação que deve assumir o valor unitário em $\boldsymbol{x}=\boldsymbol{x}_{i}$. Nas análises numéricas adotou-se a seguinte função exponencial:

$$
g\left(\boldsymbol{x}-\boldsymbol{x}_{i}\right)=\left\{\begin{array}{cc}
e^{-\left(\frac{2\left(\boldsymbol{x}-\boldsymbol{x}_{i}\right)}{r_{n l}}\right)^{2}} & \text { para } \boldsymbol{x}-\boldsymbol{x}_{i} \leq r_{n l} \\
0 & \text { para } \boldsymbol{x}-\boldsymbol{x}_{i}>r_{n l}
\end{array}\right.
$$

A implementação numérica da análise não-local é relativamente simples, substituindose a forma integral da expressão (4.21) pela razão entre somatórios, tem-se a deformação equivalente em um determinado ponto de integração $\boldsymbol{x}_{i}$ dada por: 


$$
\bar{\varepsilon}_{e q}\left(\boldsymbol{x}_{i}\right)=\frac{\sum_{\boldsymbol{x}_{j} \in V\left(r_{n l}\right)} g\left(\boldsymbol{x}_{j}-\boldsymbol{x}_{i}\right) w\left(\boldsymbol{x}_{j}\right) \varepsilon_{e q}(\boldsymbol{x})}{\sum_{\boldsymbol{x}_{j} \in V\left(r_{n l}\right)} g\left(\boldsymbol{x}_{j}-\boldsymbol{x}_{i}\right) w\left(\boldsymbol{x}_{j}\right)}
$$

Observe que $\boldsymbol{x}_{j}$ corresponde aos pontos da integração numérica pertencentes à região definida pelo raio $r_{n l}$ e com centro em $\boldsymbol{x}_{i}$. A definição desses pontos pode ser feita em um pré-processamento, para evitar que, a todo momento de cálculo do dano, as vizinhanças de cada ponto sejam novamente construídas. Já $w\left(\boldsymbol{x}_{j}\right)$ refere-se à parcela de área (ou volume) do domínio vinculada ao ponto $\boldsymbol{x}_{j}$. Tal parcela corresponde ao peso do ponto $\boldsymbol{x}_{j}$ na quadratura de Gauss multiplicado pelo respectivo jacobiano.

Ainda em DAVENNE; SAOURIDIS; PIAU (1989), observa-se que algum cuidado deve ser tomado em análises em que a simetria da geometria e do carregamento é explorada. Como se mostra na Figura 4.4, pontos fictícios devem ser considerados, para que a resposta numérica seja equivalente àquela que seria obtida para uma análise em que toda a estrutura esteja representada.

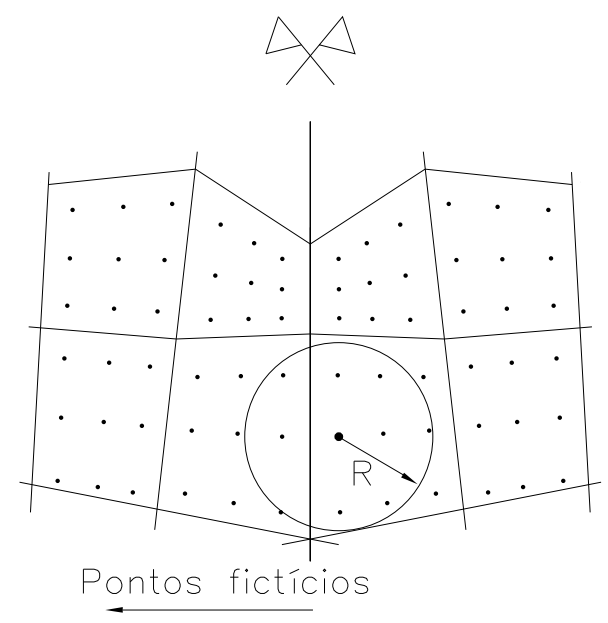

Figura 4.4: Análise não-local em estrutura com simetria 


\section{Capítulo 5}

\section{Experimentos Numéricos em Análise Não-Linear}

Este capítulo reúne aplicações dos métodos sem malha, no caso o Método das Nuvens $h p$, e do MEFG no estudo do comportamento não-linear de estruturas, decorrente do fenômeno de danificação do meio. Assim como no capítulo 3, procura-se evidenciar aspectos de implementação, especialmente aqueles introduzidos para a adequação dos métodos aos modelos constitutivos admitidos. Para isso, são apresentadas as análises de dois problemas. O primeiro deles corresponde a uma viga em concreto armado e é resolvido pelo Método das Nuvens $h p$ e pelo MEFG. O segundo problema corresponde a uma chapa de concreto submetida a deslocamentos impostos, através da qual procura-se avaliar a capacidade do enriquecimento polinomial do MEFG para aproximar a presença da localização do dano.

A escolha do Método das Nuvens $h p$ explica-se por abranger, em sua formulação, o MGLE. Para a simulação da resposta não-linear da estrutura são empregados os dois modelos constitutivos, Mazars e La Borderie, descritos anteriormente. Seguindo-se a mesma abordagem da seção 3.1, a representação adotada é uni-axial. Os resultados de análises estática e dinâmica são apresentados salientando-se não apenas detalhes da formulação numérica como também algumas interessantes discussões sobre o comportamento do material.

As análises estáticas feitas através do MEFG são bi-dimensionais, a exemplo dos problemas da seção 3.2. Devido à simplicidade de implementação e pelo fato de não haver inversão de carregamento, emprega-se o Modelo de Mazars para a simulação do comportamento do material. Aspectos de implementação do método para a análise não-linear são discutidos juntamente com os resultados encontrados. 


\subsection{Viga em Concreto Armado}

O problema selecionado encontra-se representado na Figura 5.1, e corresponde a uma viga de concreto armado bi-apoiada, com seção transversal retangular e submetida a duas forças verticais $F$ posicionadas simetricamente com relação ao meio do vão. $\mathrm{O}$ momento constante, que solicita a região entre as duas forças, induz uma distribuição difusa de micro-fissuras ao longo deste trecho. O mecanismo de deterioração do meio é, portanto, bastante semelhante àquele esperado para a simulação pelos modelos de dano contínuo, capítulo 4.

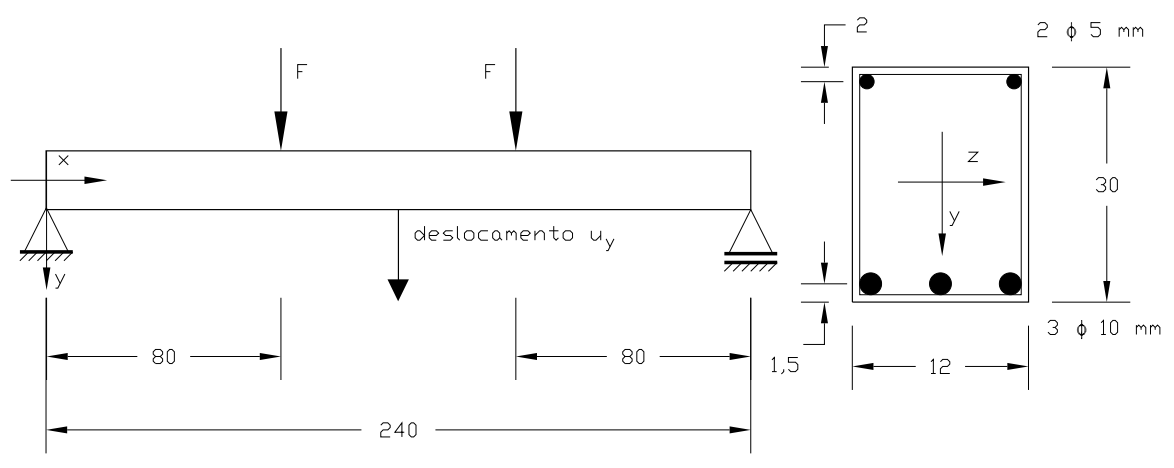

Figura 5.1: Viga em concreto armado - geometria e armação - medidas em cm

Na tabela 5.1, são apresentados os dados relativos às propriedades do aço e do concreto e entre eles, os parâmetros dos modelos de Mazars e de La Borderie que caracterizam o meio:

\begin{tabular}{|c|c|c|c|}
\hline$A_{T}=0,995$ & $B_{T}=8000$ & $A_{C}=0,85$ & $B_{C}=1050$ \\
\hline$\varepsilon_{d 0}=0,00007$ & $E_{c}=29200 \mathrm{MPa}$ & $E_{s}=196000 \mathrm{MPa}$ & $\nu_{c}=0,2$ \\
\hline$\nu_{s}=0,3$ & $Y_{o 1}=3,05 \cdot 10^{-4} \mathrm{MPa}$ & $A_{1}=3,50 \cdot 10^{3} \mathrm{MPa}^{-1}$ & $B_{1}=0,95$ \\
\hline$\beta_{1}=1,0 \mathrm{MPa}$ & $Y_{o 2}=5,0 \cdot 10^{-3} \mathrm{MPa}$ & $A_{2}=6,80 \mathrm{MPa}^{-1}$ & $B_{2}=0,7705$ \\
\hline$\beta_{2}=-10,0 \mathrm{MPa}$ & $\sigma_{f}=2,60 \mathrm{MPa}$ & $\rho_{c}=2500 \mathrm{~kg} / \mathrm{m}^{3}$ & $\rho_{s}=7850 \mathrm{~kg} / \mathrm{m}^{3}$ \\
\hline
\end{tabular}

Tabela 5.1: Parâmetros do material $(c \rightarrow$ concreto e $s \rightarrow$ aço)

onde $\rho_{c}$ e $\rho_{s}$ correspondem à densidade do concreto e do aço respectivamente. Os parâmetros para o Modelo de Mazars foram obtidos por identificação paramétrica em ensaios apresentados em ÁLVARES (1993), onde também se encontram os resultados experimentais utilizados para confronto. Já os parâmetros utilizados no Modelo de La Borderie foram determinados ajustando-os às curvas de tensão e compressão axial do Modelo de Mazars, PITUBA; PROENÇA; ÁlVARES (1999). Com relação ao aço, considerou-se o comportamento perfeitamente elástico. 


\subsection{Análise pelo Método das Nuvens $h p$}

A Figura 5.2(a) representa as condições de contorno e a discretização adotadas para a análise com o Método das Nuvens. Por se tratar de uma análise uni-axial arbitrou-se que os apoios e as cargas estivessem aplicados sobre o eixo $x$, posicionado sobre o centróide da seção transversal retangular.

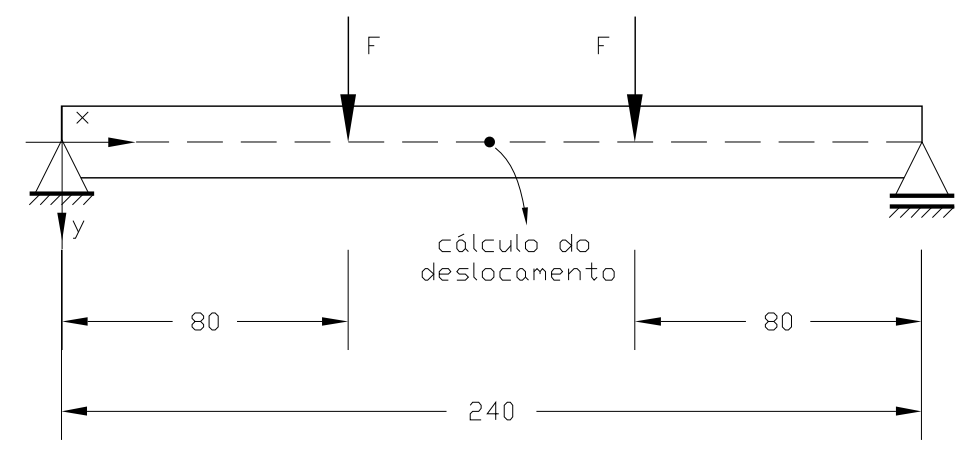

(a) Geometria e condições de contorno adotadas

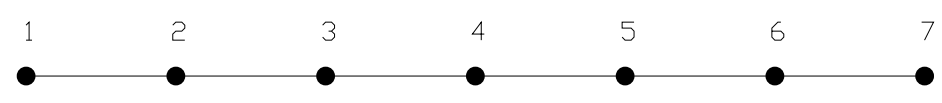

(b) Discretização

Figura 5.2: Abordagem uni-axial

A hipótese cinemática é a da teoria de vigas de Bernoulli para um campo de deformações dado pela combinação das derivadas primeira e segunda do campo de deslocamentos, $\varepsilon_{x}=u_{x}^{\prime}-y u_{y}^{\prime \prime}$. O funcional de energia, em uma análise dinâmica sem amortecimento, resulta:

$$
\begin{aligned}
& \Pi=\overbrace{\int_{0}^{L}\left\{\int_{0}^{h} E\left(u_{x}^{\prime}-y u_{y}^{\prime \prime}\right)^{2} d y\right\} l_{z} d x}^{\mathcal{U}}-\overbrace{\left(\left.F \cdot u_{y}\right|_{x=a}+\left.F \cdot u_{y}\right|_{x=L-a}\right)}^{\mathcal{W}_{\mathrm{ext}}} \\
& -\underbrace{\int_{0}^{L}\left\{\int_{0}^{h} u_{x} \rho \ddot{u}_{x}+u_{y} \rho \ddot{u}_{y} d y\right\} l_{z} d x}_{\mathcal{W}_{i n}}
\end{aligned}
$$


onde $u_{x}$ e $u_{y}$ são os deslocamentos dos pontos localizados no eixo, nas direções $x$ e $y$ respectivamente, $l_{z}$ é a espessura, $L$ representa o comprimento da viga e $\mathcal{W}_{i n}$ é o trabalho devido às forças inerciais, consideradas como forças de volume pelo princípio de d'Alembert, BATHE (1996). As condições de contorno essenciais são $\left.u_{x}\right|_{x=0}=0$ e $\left.u_{y}\right|_{x=0}=\left.u_{y}\right|_{x=L}=0$. Para a análise dinâmica, deve-se fazer também com que as acelerações $\left.\ddot{u}_{x}\right|_{x=0}=0$ e $\left.\ddot{u}_{y}\right|_{x=0}=\left.\ddot{u}_{y}\right|_{x=L}=0$ no instante de tempo $t=0$.

Aplicando-se o princípio da estacionaridade e introduzindo-se as expressões aproximadas dos deslocamentos vertical e horizontal, obtidas do MMQM, (2.6), e da família de Nuvens- $h p$ (2.16), chega-se à seguinte aproximação de Galerkin para a forma variacional do $\mathrm{PVC}$, em $t=0$ :

$$
\begin{aligned}
& \delta \bar{\Pi}=\int_{0}^{L}\left\{\delta \boldsymbol{U}_{x}^{T} \boldsymbol{\Phi}_{x}^{\prime} \boldsymbol{\Phi}_{x}^{\prime T} \boldsymbol{U}_{x} \int_{0}^{H} E b d y+\delta \boldsymbol{U}_{x}^{T} \boldsymbol{\Phi}_{x}^{\prime} \boldsymbol{\Phi}_{y}^{\prime \prime T} \boldsymbol{U}_{y} \int_{0}^{H} E y l_{z} d y\right. \\
& \left.+\delta \boldsymbol{U}_{y}^{T} \boldsymbol{\Phi}_{y}^{\prime \prime} \boldsymbol{\Phi}_{x}^{\prime T} \boldsymbol{U}_{x} \int_{0}^{H} E y l_{z} d y+\delta \boldsymbol{U}_{y}^{T} \boldsymbol{\Phi}_{y}^{\prime \prime} \boldsymbol{\Phi}_{y}^{\prime \prime T} \boldsymbol{U}_{y} \int_{0}^{H} E y^{2} l_{z} d y\right\} d x \\
& -\left.\left(\delta \boldsymbol{U}_{y}\right)^{T} \Phi_{y}\right|_{x=0} P-\left.\left(\delta \boldsymbol{U}_{y}\right)^{T} \Phi_{y}\right|_{x=L} P \\
& -\int_{0}^{L}\left\{\delta \ddot{\boldsymbol{U}}_{x}^{T} \boldsymbol{\Phi}_{x} \boldsymbol{\Phi}_{x}^{T} \ddot{\boldsymbol{U}}_{x} \int_{0}^{H} E l_{z} d y+\delta \ddot{\boldsymbol{U}}_{x}^{T} \boldsymbol{\Phi}_{x} \boldsymbol{\Phi}_{y}^{\prime T} \ddot{\boldsymbol{U}}_{y} \int_{0}^{H} E y l_{z} d y\right. \\
& \left.+\delta \ddot{\boldsymbol{U}}_{y}^{T} \boldsymbol{\Phi}_{y}^{\prime} \boldsymbol{\Phi}_{x}^{T} \ddot{\boldsymbol{U}}_{x} \int_{0}^{H} E y l_{z} d y+\delta \ddot{\boldsymbol{U}}_{y}^{T} \boldsymbol{\Phi}_{y}^{\prime} \boldsymbol{\Phi}_{y}^{\prime T} \ddot{\boldsymbol{U}}_{y} \int_{0}^{H} E y^{2} l_{z} d y\right\} d x \\
& +\left.\left(\delta \lambda_{1}^{u_{x}}\right)^{T} \boldsymbol{\Phi}^{T} \boldsymbol{U}_{x}\right|_{x=0}+\left.\delta \boldsymbol{U}_{x}^{T} \boldsymbol{\Phi} \lambda_{1}^{u_{x}}\right|_{x=0} \\
& +\left.\delta \lambda_{1}^{u_{y}} \boldsymbol{\Phi}^{T} \boldsymbol{U}_{y}\right|_{x=0}+\left.\delta \boldsymbol{U}_{y}^{T} \boldsymbol{\Phi} \lambda_{1}^{u_{y}}\right|_{x=0} \\
& +\left.\lambda_{N}^{u_{x}} \boldsymbol{\Phi}^{T} \boldsymbol{U}_{x}\right|_{x=L}+\left.\delta \boldsymbol{U}_{x}^{T} \boldsymbol{\Phi} \lambda_{N}^{u_{x}}\right|_{x=L} \\
& +\left.\left(\delta \ddot{\lambda}_{1}^{u_{x}}\right)^{T} \boldsymbol{\Phi}^{T} \ddot{\boldsymbol{U}}_{x}\right|_{x=0}+\left.\delta \ddot{\boldsymbol{U}}_{x}^{T} \boldsymbol{\Phi} \ddot{\lambda}_{1}^{u_{x}}\right|_{x=0} \\
& +\left.\left(\delta \ddot{\lambda}_{1}^{u_{y}}\right)^{T} \boldsymbol{\Phi}^{T} \ddot{\boldsymbol{U}}_{y}\right|_{x=0}+\left.\delta \ddot{\boldsymbol{U}}_{y}^{T} \boldsymbol{\Phi} \ddot{\lambda}_{1}^{u_{y}}\right|_{x=0} \\
& +\left.\left(\delta \ddot{\lambda}_{N}^{u_{x}}\right)^{T} \boldsymbol{\Phi}^{T} \ddot{\boldsymbol{U}}_{x}\right|_{x=L}+\left.\delta \ddot{\boldsymbol{U}}_{x}^{T} \boldsymbol{\Phi} \ddot{\lambda}_{N}^{u_{x}}\right|_{x=L}=0
\end{aligned}
$$

onde $H$ representa a altura da viga e os subscritos $x$ e $y$ particularizam os vetores generalizados dos parâmetros nodais, das funções de aproximação, e dos multiplicadores de Lagrange (estes relacionados aos nós extremos 1 e N), com relação às direções ho- 
rizontal e vertical, respectivamente. Nota-se que, além dos parâmetros nodais $\boldsymbol{U}_{x} \mathrm{e}$ $\boldsymbol{U}_{y}$, também encontram-se aproximadas e, da mesma forma, suas derivadas segundas com relação ao tempo, representadas por $\ddot{\boldsymbol{U}}_{x}$ e $\ddot{\boldsymbol{U}}_{y}$. Para a imposição dos valores iniciais do vetor de acelerações generalizadas, $\ddot{a}$, o procedimento é o mesmo que para as condições de contorno, empregando-se a segunda taxa de variação no tempo dos multiplicadores de Lagrange, ${\ddot{\lambda_{1}}}^{u_{x}},{\ddot{\lambda_{1}}}^{u_{y}},{\ddot{\lambda_{N}}}^{u_{x}}$ e $\ddot{\lambda_{N}}{ }^{u_{y}}$.

Simplificando-se, então, a equação (5.2), chega-se ao seguinte sistema de equações:

$$
\begin{aligned}
& \widehat{\boldsymbol{K}} \text { de ordem }\left[\left(2 N^{*}+3\right) \times\left(2 N^{*}+3\right)\right] \quad \widehat{\boldsymbol{U}} \text { de ordem }\left[2 N^{*}+3\right] \quad \widehat{\boldsymbol{F}} \text { de ordem }\left[2 N^{*}+3\right]
\end{aligned}
$$

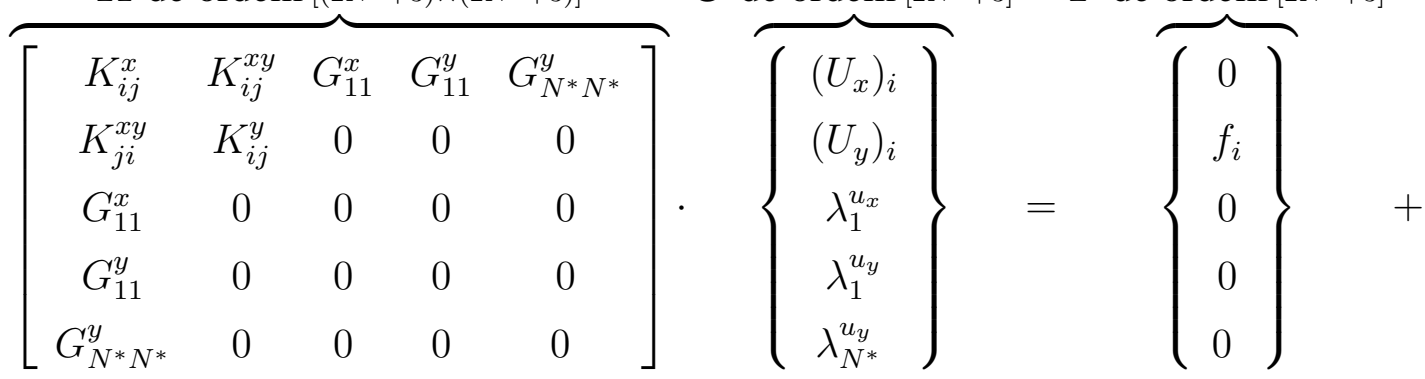

$$
\begin{aligned}
& \underbrace{\left[\begin{array}{ccccc}
M_{i j}^{x} & M_{i j}^{x y} & H_{11}^{x} & H_{11}^{y} & H_{N^{*} N^{*}}^{y} \\
M_{j i}^{x y} & M_{i j}^{y} & 0 & 0 & 0 \\
H_{11}^{x} & 0 & 0 & 0 & 0 \\
H_{11}^{y} & 0 & 0 & 0 & 0 \\
H_{N^{*} N^{*}}^{y} & 0 & 0 & 0 & 0
\end{array}\right]}_{\widehat{\boldsymbol{M}} \text { de ordem }[(2 N+3) \times(2 N+3)]} \cdot \underbrace{\left\{\begin{array}{c}
\left(\ddot{U}_{x}\right)_{i} \\
\left(\ddot{U}_{y}\right)_{i} \\
\ddot{\lambda}_{1}^{u_{x}} \\
\ddot{\lambda}_{1}^{u_{y}} \\
\ddot{\lambda}_{N^{*}}^{u_{y}}
\end{array}\right\}}_{\widehat{\boldsymbol{U}}}
\end{aligned}
$$

em que:

$$
\begin{gathered}
K_{i j}^{x}=\int_{0}^{L}\left\{\boldsymbol{\Phi}_{x}^{\prime} \boldsymbol{\Phi}_{x}^{\prime T} \int_{0}^{h} E l_{z} d y\right\} d x \quad K_{i j}^{y}=\int_{0}^{L}\left\{\boldsymbol{\Phi}_{y}^{\prime \prime} \boldsymbol{\Phi}_{y}^{\prime \prime T} \int_{0}^{h} E y^{2} b d y\right\} d x \\
K_{i j}^{x y}=\int_{0}^{L}\left\{\delta \boldsymbol{\Phi}_{y}^{\prime \prime} \boldsymbol{\Phi}_{x}^{\prime T} \int_{0}^{h} E y l_{z} d y\right\} d x \quad f_{i}=P\left(\left.\Phi_{y}\right|_{x=a}+\left.\Phi_{y}\right|_{x=L-a}\right) \\
M_{i j}^{x}=\int_{0}^{L}\left\{\boldsymbol{\Phi}_{x} \boldsymbol{\Phi}_{x}^{T} \int_{0}^{h} E b d y\right\} d x \quad M_{i j}^{y}=\int_{0}^{L}\left\{\boldsymbol{\Phi}_{y}^{\prime} \boldsymbol{\Phi}_{y}^{\prime T} \int_{0}^{h} E y^{2} l_{z} d y\right\} d x
\end{gathered}
$$




$$
\begin{gathered}
M_{i j}^{x y}=\int_{0}^{L}\left\{\delta \Phi_{y}^{\prime} \boldsymbol{\Phi}_{x}^{T} \int_{0}^{h} E y l_{z} d y\right\} d x \quad G_{11}^{x}=\Phi_{1}^{x}(0) \\
G_{11}^{y}=\Phi_{1}^{y}(0) \quad G_{N^{*} N^{*}}^{y}=\Phi_{1}^{y}(L) \\
H_{11}^{x}=\Phi_{1}^{x}(0) \quad H_{11}^{y}=\Phi_{1}^{y}(0) \quad H_{N^{*} N^{*}}^{y}=\Phi_{1}^{y}(L)
\end{gathered}
$$

para $i, j=1, \cdots, N^{*}$, onde $N^{*}$, assim com no exemplo da seção 3.1, depende do grau de enriquecimento escolhido para a análise e a matriz $M$ recebe a denominação de matriz de massa consistente. Observa-se, ainda, que a relação constitutiva está sendo representada pela variável $E$, que assume valores iguais ao módulo de elasticidade, $E_{0}$, para o material íntegro ou a um valor penalizado pelo dano, $E=(1-D) E_{0}$, nos pontos em que o material encontra-se deteriorado.

\subsubsection{Problema Não-Linear}

Em análise não-linear, procura-se estabelecer, para cada instante de tempo $t$ de aplicação do carregamento, o equilíbrio entre os vetores das forças generalizadas externa, ${ }^{t} \widehat{\boldsymbol{F}}_{\text {ext }}=\widehat{\boldsymbol{F}}+\widehat{\boldsymbol{M}} \widehat{\ddot{\boldsymbol{U}}}$ e interna, ${ }^{t} \widehat{\boldsymbol{F}}_{\text {int }}$, esse obtido a partir das tensões calculadas nos elementos e definido como:

$$
\widehat{\boldsymbol{F}}_{\mathrm{int}}^{T}=\left[\int_{0}^{L} \int_{0}^{h} \boldsymbol{\Phi}_{x}^{\prime} \sigma_{x} b d y d x-\int_{0}^{L} \int_{0}^{h} y \boldsymbol{\Phi}_{y}^{\prime \prime} \sigma_{x} b d y d x \quad 0 \quad 00 \quad 0\right]-\underbrace{\widehat{\boldsymbol{M}} \ddot{\boldsymbol{a}}}_{\text {forças inerciais }}
$$

Dessa forma, deve-se ter:

$$
{ }^{t} \widehat{\boldsymbol{F}}_{\text {ext }}-{ }^{t} \widehat{\boldsymbol{F}}_{\text {int }}=\mathbf{0}
$$

No procedimento de resolução do método de Newton-Raphson, BATHE (1996), assume-se como conhecida a solução ${ }^{t} \widehat{\boldsymbol{U}}$ para um instante $t$ e procura-se verificar o equilíbrio em $t+\Delta t$. As equações usadas no processo iterativo são as seguintes: 


$$
\begin{aligned}
\text { Para } i_{t}=1,2,3, \cdots & \\
\boldsymbol{\psi}^{\left(i_{t}-1\right)} & ={ }^{t+\Delta t} \widehat{\boldsymbol{F}}_{\mathrm{ext}}-{ }^{t+\Delta t} \widehat{\boldsymbol{F}}_{\mathrm{int}}^{\left(i_{t}-1\right)} \\
{ }^{t+\Delta t} \widehat{\boldsymbol{K}}_{\mathrm{tg}}^{\left(i_{t}-1\right)}{ }^{\Delta \widehat{\boldsymbol{U}}^{\left(i_{t}\right)}} & =\boldsymbol{\psi}^{\left(i_{t}-1\right)} \\
{ }^{t+\Delta t} \widehat{\boldsymbol{U}}^{\left(i_{t}\right)} & ={ }^{t+\Delta t} \widehat{\boldsymbol{U}}^{\left(i_{t}-1\right)}+\Delta \widehat{\boldsymbol{U}}^{\left(i_{t}\right)}
\end{aligned}
$$

tendo como condições iniciais:

$$
{ }^{t+\Delta t} \widehat{\boldsymbol{U}}^{(0)}={ }^{t} \widehat{\boldsymbol{U}} ; \quad{ }^{t+\Delta t} \widehat{\boldsymbol{K}}_{\mathrm{tg}}^{(0)}={ }^{t} \widehat{\boldsymbol{K}}_{\mathrm{tg}} ; \quad{ }^{t+\Delta t} \widehat{\boldsymbol{F}}^{(0)}={ }^{t} \widehat{\boldsymbol{F}}
$$

em que:

$$
{ }^{t} \widehat{\boldsymbol{K}}_{\mathrm{tg}}=\frac{\partial^{t} \widehat{\boldsymbol{F}}}{\partial^{t} \widehat{\boldsymbol{U}}}
$$

é a forma tangente da matriz de rigidez ${ }^{1}$.

Com a primeira equação de (5.6), calcula-se o desequilíbrio, $\boldsymbol{\psi}^{\left(i_{t}-1\right)}$ entre os vetores de força ${ }^{t+\Delta t} \widehat{\boldsymbol{F}}_{\text {ext }} \mathrm{e}^{t+\Delta t} \widehat{\boldsymbol{F}}_{\text {int }}^{\left(i_{t}-1\right)}$ que, na segunda equação, produz o acréscimo de deslocamentos, $\Delta \widehat{\boldsymbol{U}}^{\left(i_{t}\right)}$. Este acréscimo nos deslocamentos é utilizado na última equação de (5.6) para a atualização do vetor dos deslocamentos até o passo de tempo $t+\Delta t,{ }^{t+\Delta t} \widehat{\boldsymbol{U}}^{\left(i_{t}\right)}$. Este processo iterativo é repetido até que $\boldsymbol{\psi}^{\left(i_{t}-1\right)}$ e/ou $\Delta \widehat{\boldsymbol{U}}^{\left(i_{t}\right)}$ sejam suficientemente pequenos.

Sob condições adequadas ${ }^{2}$, o emprego da forma tangente da matriz de rigidez permite com que se tenha uma convergência quadrática para o método de NewtonRaphson. Se no lugar da forma tangente for utilizada a forma secante, ${ }^{t} \widehat{\boldsymbol{K}}_{\text {sec }}$, tal eficiência é perdida e a convergência pode se tornar bastante lenta.

\subsubsection{Integração Numérica na Seção Transversal}

Nas equações (5.2) e (5.4), estão presentes as integrais nas direções $x$ e $y$. As primeiras são calculadas numericamente através da quadratura de Gauss-Legendre. Para problemas lineares, em que não ocorrem variações nas propriedades do material, as integrais em $y$ são normalmente calculadas analiticamente. Para a presente análise, seja através do modelo de Mazars ou La Borderie, o dano penaliza a rigidez da estrutura tanto na direção longitudinal quanto ao longo da altura da viga. Não há, por essa

\footnotetext{
${ }^{1}$ Maiores detalhes da construção da matriz de rigidez tangente ou mesmo sua simplificação em matriz secante podem ser encontrados no apêndice A.

${ }^{2}$ Tais condições são descritas em BATHE (1996) e consistem em se ter ${ }^{t} \widehat{\boldsymbol{K}}_{\text {tg }}$ não singular e satisfazendo a continuidade de Lipschitz, além de se ter um passo de tempo suficientemente pequeno.
} 
razão, como calcular analiticamente as integrais ao longo de $y$ nas equações (5.2) e (5.4). Emprega-se, então, uma técnica de integração ao longo da altura, utilizada, por exemplo, em ÁLVARES (1993). A seção transversal da viga em cada elemento é dividida em camadas, também denominadas estratos e, para cada uma, adota-se como constante o valor penalizado de $E=E_{0}(1-D)$ calculado em seu centróide. Quanto maior o número de camadas, maior a precisão da integração em y. Em PAULA (2001), discute-se melhor esse assunto, chegando-se à conclusão que é necessário um número superior a 10 estratos para que se garanta a qualidade da resposta numérica.

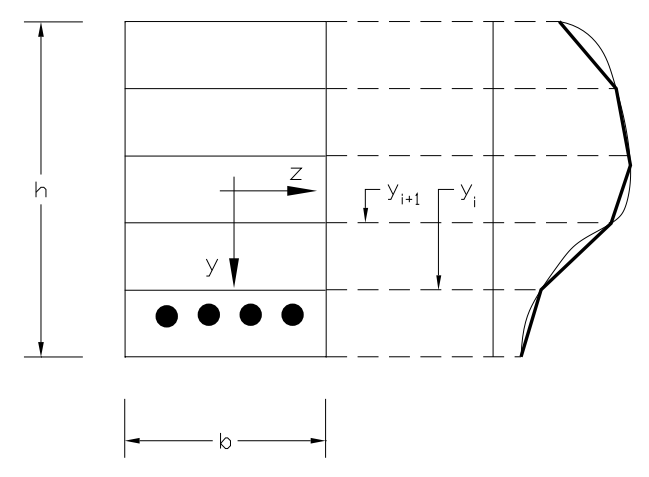

Figura 5.3: Sistema de estratos

Na Figura 5.3, encontra-se representada a seção transversal da viga dividida em estratos. Nota-se que, diferentemente da técnica utilizada por ÁLVARES (1993) e PAULA (2001), emprega-se uma variação linear para $E$ ao longo de cada camada. Obtém-se, assim, uma melhor aproximação para a integração ao longo da altura. No contexto do Método das Nuvens $h p$, por não serem definidos elementos, a divisão em camadas é realizada em cada célula de integração. A integração em $y$ das expressões (5.2) e (5.4) pode, então, ser reformulada considerando-se:

$$
\begin{aligned}
\int_{0}^{h} E l_{z} d y= & \sum_{i=1}^{n} \frac{l_{z}}{2}\left(E_{i+1}+E_{i}\right)\left(y_{i}-y_{i+1}\right) \\
\int_{0}^{h} E y l_{z} d y= & \sum_{i=1}^{n} \frac{l_{z}}{6}\left[\left(E_{i+1}\left(y_{i}^{2}+y_{i} y_{i+1}-2 y_{i+1}^{2}\right)+\right.\right. \\
& \left.+E_{i}\left(2 y_{i}^{2}+y_{i} y_{i+1}-y_{i+1}^{2}\right)\right] \\
\int_{0}^{h} E y^{2} l_{z} d y= & \sum_{i=1}^{n} \frac{l_{z}}{12}\left[\left(E_{i+1}\left(y_{i}^{3}+y_{i}^{2} y_{i+1}+y_{i} y_{i+1}^{2}-3 y_{i+1}^{2}\right)+\right.\right. \\
& \left.+E_{i}\left(3 y_{i}^{3}-y_{i}^{2} y_{i+1}-y_{i} y_{i+1}^{2}-y_{i+1}^{2}\right)\right]
\end{aligned}
$$


onde $E_{i}=E_{0}\left(1-D_{i}\right)$ e $D_{i}$ corresponde ao dano calculado em $y=y_{i}$.

O aço, por sua vez, é considerado, separadamente, como um meio puramente elástico. As barras da armadura que ocupam uma certa posição y são substituídas por uma camada de aço equivalente, sobreposta às camadas de concreto.

\subsubsection{Análise Numérica}

Para a realização da análise numérica pelo Método das Nuvens $h p$, adotou-se:

ordem da base polinomial: no sistema de equações (5.3) aparecem derivadas de segunda ordem das funções de forma $\Phi_{y}$ e de primeira ordem para $\Phi_{x}$. Para que a convergência da solução esteja garantida (completidade), as aproximações de $u_{y}$ e $u_{x}$ devem representar no mínimo polinômios completos do segundo e primeiro grau respectivamente. Sendo assim, para a presente análise foram utilizadas as famílias de Nuvens- $h p \Im_{N}^{k=0, p=3}$ e $\Im_{N}^{k=0, p=1}$ para as aproximações nas direções $y$ e $x$. Tais famílias foram construídas a partir das funções de Shepard, 2.13, enriquecidas pelas bases de monômios $\boldsymbol{P}_{p=3}=\left\{\begin{array}{llll}1 & x & x^{2} & x^{3}\end{array}\right\}$ e $\boldsymbol{P}_{p=1}=\left\{\begin{array}{ll}1 & x\end{array}\right\}$

distribuição de pontos: foi empregada uma distribuição uniforme de pontos, mostrada na Figura 5.2(b), com a distância entre pontos $h=40 \mathrm{~cm}$. A função de ponderação empregada para a construção das funções de Shepard é a do tipo spline cúbica, já empregada na seção 3.1.1, com $R_{j}=h, \forall j$;

integração numérica: para melhor captar a distribuição do dano, foram empregados 20 pontos de quadratura por célula para a integração numérica de GaussLegendre ao longo de $x$, e 10 estratos para a integração na altura $y$;

não-linearidade física: apenas o concreto tem comportamento não-linear, simulado pelos modelos de Mazars e La Borderie. O aço foi considerado elástico linear e perfeitamente aderente ao concreto. O problema não-linear físico foi resolvido aplicando-se o Método de Newton-Raphson, BATHE (1996). Para o Modelo de Mazars foi empregada a formulação tangente, expressão (4.6) e para o Modelo de La Borderie a secante, (4.20);

análise dinâmica: a solução do sistema de equações (5.3) foi obtida através do método de integração implícita de Newmark, adotando-se o algoritmo utilizado em 
ARGYRIS; MLEJNEK (1991) para análise não-linear física. Detalhes da implementação desse algoritmo, em que são combinados os procedimentos de Newmark e de Newton-Raphson, podem ser encontrados em RODRIGUES (1997) e PAULA (2001);

equilíbrio: a convergência do algoritmo em cada passo incremental foi averiguada pela norma de energia incremental, com tolerância de $10^{-10}$ e da norma $L_{2}$ do resíduo dos deslocamentos, com tolerância relativa de $10^{-6}$;

simetria: as análises numéricas foram conduzidas para a estrutura completa, sem que a simetria fosse considerada. Tal fato se deve à análise dinâmica e não a alguma limitação do método sem malha.

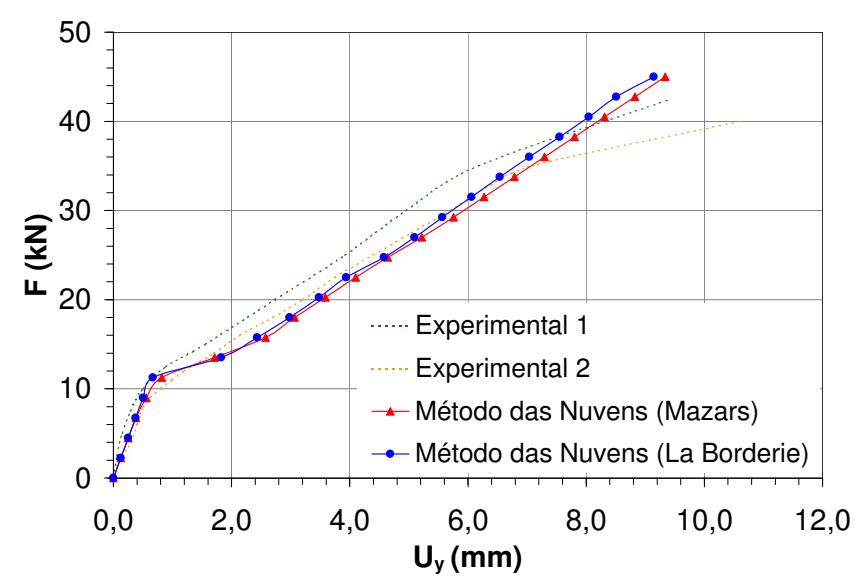

Figura 5.4: Análises estáticas - resultados experimentais de ÁLVARES (1993)

No gráfico da Figura 5.4 confrontam-se os resultados experimentais e numéricos para a análise estática. Para a solução do problema não-linear com ambos os modelos, Mazars e La Borderie, foram utilizados 20 passos de força no valor de $2,25 \mathrm{kN}$. A divergência das curvas após o nível de força de $35 \mathrm{kN}$ ocorre devido à plastificação das barras, que não é considerada nas análises numéricas. Para valores inferiores, os resultados podem ser considerados bons, observando-se uma equivalência entre a resposta obtida para os dois modelos adotados.

Para o problema dinâmico, adotou-se um passo de tempo $\Delta t=1 \cdot 10^{-5} \mathrm{~s}$ e foram consideradas duas situações de solicitação, vibração forçada, Figura 5.5(a), para uma força $F(t)=25 \mathrm{kN}$, de $t=0$ a $t \rightarrow \infty$ e vibração livre, Figura 5.5(b), para um 
deslocamento inicial de $1 \mathrm{~mm}$. Nestas duas situações, as respostas não-lineares da estrutura foram contrapostas à resposta elástica-linear sem dano. Devido ao processo de danificação, observa-se um aumento no período de vibração, com ambos os modelos e para os dois tipos de solicitação. Este aumento é, contudo, mais pronunciado para o Modelo de Mazars. Para o caso de vibração livre, a diferença entre as respostas dos dois modelos é ainda maior. Para a análise feita com o Modelo de La Borderie, observou-se uma redução bastante significativa da amplitude da curva, que passa a vibrar ao redor de uma posição diferente da de repouso.

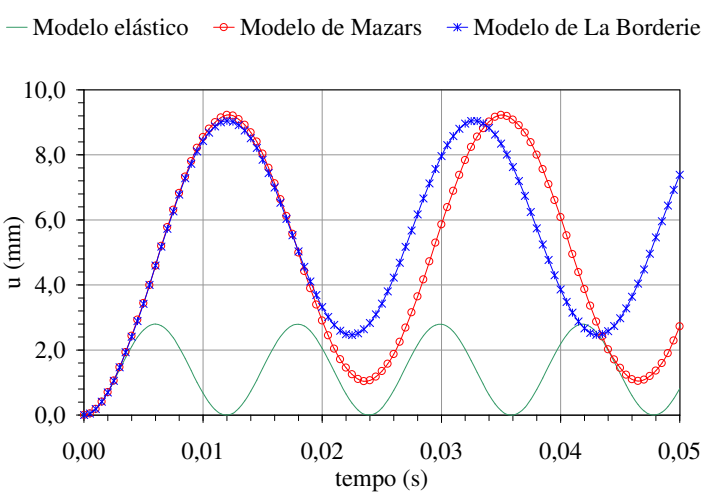

(a) Vibração forçada

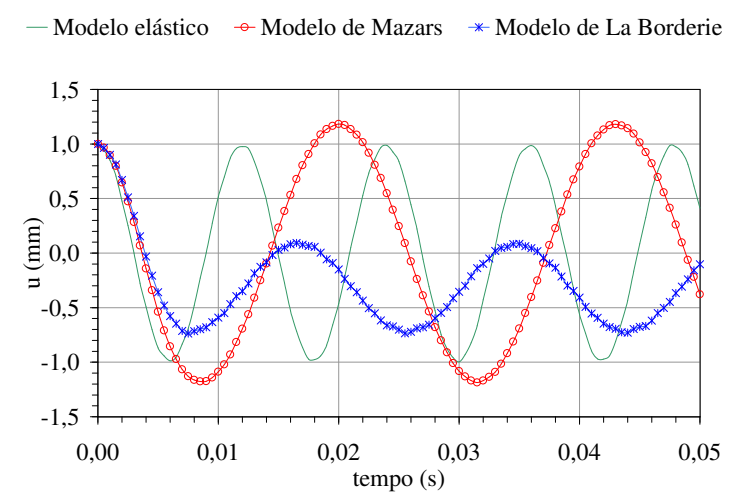

(b) Vibração livre

Figura 5.5: Análises dinâmicas

Dois fatores são importantes para explicar as respostas numéricas obtidas. Primeiramente, deve-se lembrar que, diferentemente do Modelo de Mazars, as deformações residuais são consideradas no Modelo de La Borderie, seção 4.3. Dessa forma, para ambos os tipos de solicitação dinâmica, após um período inicial de evolução do dano, a estrutura governada pelo Modelo de Mazars comporta-se como um meio elástico com uma rigidez inferior à inicial. Para a simulação através do Modelo de La Borderie, a vibração livre passa a ocorrer em uma nova posição, definida pelo estado de deformações residuais. Outro fator importante refere-se ao comportamento uni-lateral do concreto que é levado em conta apenas no Modelo de La Borderie. Nesse caso, o fechamento das fissuras inibe a evolução do processo de danificação o que explica as menores amplitudes de vibração observadas nos dois gráficos. Este efeito é melhor observado na análise da vibração livre, pois nessa situação ocorre não apenas o descarregamento, como também a inversão da solicitação. A estrutura recupera a rigidez para um nível inferior de energia e, por isso, vibra menos. Uma discussão bem mais deta- 
lhada sobre esse tema pode ser encontrada em PAULA (2001), onde a viga de concreto armado é analisada pelo MEF, com os mesmos modelos constitutivos, incluindo-se o amortecimento do material e o comportamento plástico da armadura. Conclui-se que, apesar da ausência da comprovação experimental para as análises dinâmicas, o Modelo de La Borderie simula de forma mais adequada o comportamento do concreto. Para uma análise estática, entretanto, em que o carregamento é proporcional e não ocorre inversão de solicitação, a equivalência entre as simulações feitas com os dois modelos e a simplicidade de implementação do Modelo de Mazars acabaram sendo decisivas para o seu emprego nas experimentações numéricas bi-dimensionais realizadas, nas próximas seções, com o MEFG.

Finalmente, resta salientar que uma análise através do MGLE não se diferencia muito do que foi realizado para o Método das Nuvens $h p$, a menos do tipo de distribuição de nós. Para que resultados semelhantes sejam obtidos com o MGLE, uma base $P_{k=3}$ deve ser adotada, construindo-se, assim, uma aproximação para polinômios de ordem cúbica. Como conseqüência, torna-se necessária a utilização de um número bem superior de nós para a aproximação do domínio.

\subsection{Análise Bi-dimensional com do MEFG}

A análise estática do problema da viga de concreto armado através do MEFG foi realizada no domínio $\mathbb{R}^{2}$. O funcional de energia e o sistema de equações resultante para o PVC são representados pelas mesmas expressões da seção 3.2. O problema não-linear, proveniente da propagação do dano, é resolvido, a exemplo da seção 5.2.1, em cada passo de carga, pelo método de Newton-Raphson através das seguintes expressões, adaptadas à estrutura do MEFG:

$$
\begin{aligned}
\text { Para } i_{t}=1,2,3, \ldots, \bar{i}_{t}, \ldots, i_{t}^{\max } & \\
\boldsymbol{\psi}^{\left(i_{t}-1\right)} & ={ }^{t+\Delta t} \boldsymbol{F}_{\mathrm{ext}}-{ }^{t+\Delta t} \boldsymbol{F}_{\mathrm{int}}^{\left(i_{t}-1\right)} \\
{ }^{t+\Delta t} \boldsymbol{K}_{\mathrm{sec}}^{\left(i_{t}-1\right)} \Delta \boldsymbol{U}^{\left(i_{t}\right)} & =\boldsymbol{\psi}^{\left(i_{t}-1\right)} \\
{ }^{t+\Delta t} \boldsymbol{U}^{\left(i_{t}\right)} & ={ }^{t+\Delta t} \boldsymbol{U}^{\left(i_{t}-1\right)}+\Delta \boldsymbol{U}^{\left(i_{t}\right)}
\end{aligned}
$$

tendo como condições iniciais:

$$
{ }^{t+\Delta t} \boldsymbol{U}^{(0)}={ }^{t} \boldsymbol{U} ; \quad{ }^{t+\Delta t} \boldsymbol{K}_{\mathrm{sec}}^{(0)}={ }^{t} \boldsymbol{K}_{\mathrm{sec}} ; \quad{ }^{t+\Delta t} \boldsymbol{F}^{(0)}={ }^{t} \boldsymbol{F}
$$


Observa-se que para a matriz de rigidez, no lugar da forma tangente, foi utilizada a forma secante ${ }^{3}$. A razão para isso está no emprego do modelo de Mazars, conforme já explicado na seção 4.2.

De forma geral, a estrutura para a solução do problema não-linear é idêntica à usada tradicionalmente no MEF. Excetua-se, é claro, a maneira com que os vetores e matrizes, presentes nas expressões acima, são montados, seção 3.2. Existem, porém, alguns detalhes em que o algoritmo da análise não-linear do MEFG diferencia-se do MEF, tendo sido reunidos no apêndice F.

\subsubsection{Análise Numérica}

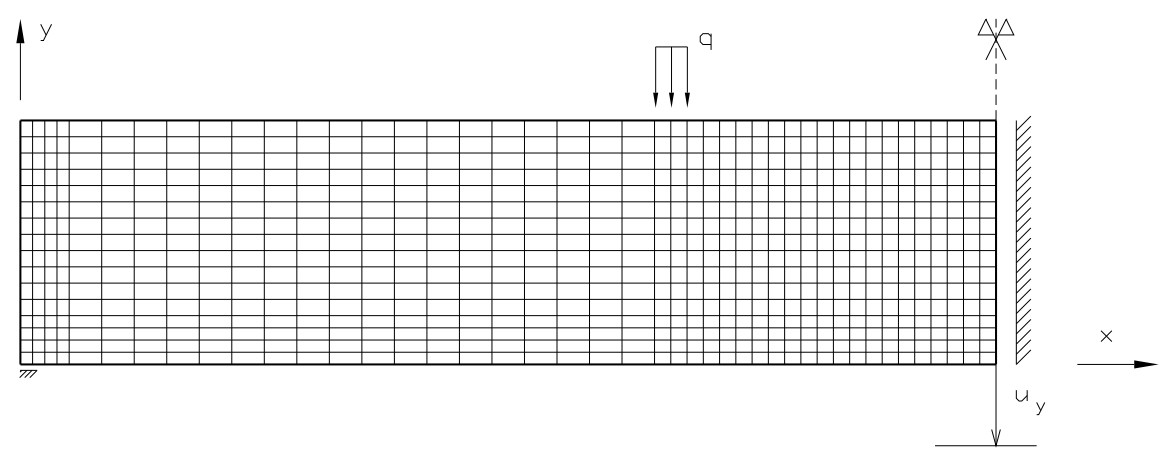

(a) Malha I - 688 elementos - NGL $=1376$

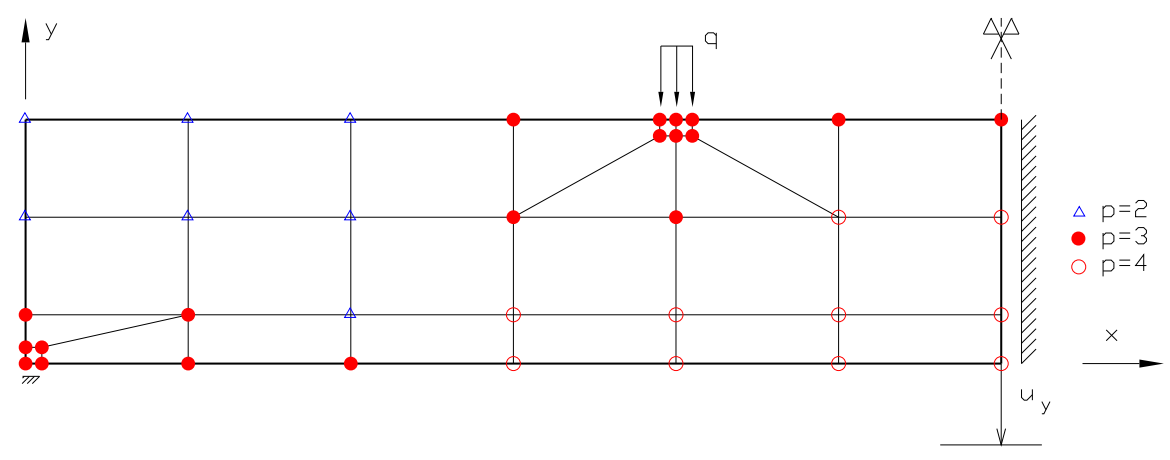

(b) Malha II - 24 elementos - NGL $=392$

Figura 5.6: Condições de contorno e malha de elementos adotadas

Duas análises estáticas bi-dimensionais foram realizadas para a viga de concreto armado da seção 5.1. As malhas de elementos finitos empregadas encontram-se representadas na Figura 5.6. Na Malha I, com um grau razoável de refinamento, a aproximação é linear e corresponde à análise convencional pelo MEF. Já na Malha II, empregada

\footnotetext{
${ }^{3}$ Ao final do apêndice A, é apresentada a expressão para a matriz de rigidez secante.
} 
na aplicação do MEFG, foram utilizados poucos elementos mas com enriquecimento nodal de ordem polinomial superior. A seguir são listadas as considerações admitidas para tais análises:

discretização: como já observado, nas Figuras 5.6(a) e 5.6(b), encontram-se representadas as malhas de elementos empregadas. O reduzido número de elementos da Malha II é compensado pelo enriquecimento polinomial de ordem máxima $p$, realizado em todas as nuvens, mas concentrado, especialmente, nas regiões em que se espera uma maior danificação, ou seja, na metade inferior da viga, entre as duas forças aplicadas;

condições de contorno: de acordo com SZABÓ; BABUŠKA (1991), as restrições nodais não são admissíveis em problemas de estado plano, exceto quando impostas para impedir os movimentos de corpo rígido em um corpo solicitado por um carregamento auto-equilibrado. Por essa razão, o apoio articulado móvel da Figura 5.1 é substituído por um apoio distribuído em uma pequena região com dimensão em $x$ de 1,5cm, como ilustram as Figuras 5.6(a) e 5.6(b). De maneira análoga e pelas mesmas razões, a força concentrada é substituída por um carregamento distribuído equivalente $q^{4}$;

simetria: a forma geométrica da viga, a distribuição do carregamento e das condições de contorno são simétricas com relação ao meio do vão; por essa razão, apenas metade da estrutura foi aproximada;

aproximação das armaduras: utilizou-se para as malhas $I$ e $I I$, a técnica de embutimento da armadura, descrita em RANJBARAN (1991) e discutida no apêndice F.2. Apenas a armadura positiva foi considerada. Testes em que a armadura negativa foi discretizada mostraram que sua contribuição à rigidez da estrutura era insignificante ${ }^{5}$. Para simular o comportamento físico adotou-se o modelo elástico-linear com perfeita aderência ao concreto;

distorção dos elementos: a Malha II foi construída tendo como base 18 elementos relativamente grandes, se comparados com a discretização da Malha I. Para a introdução das condições de contorno, seis novos elementos de dimensões bastante

\footnotetext{
${ }^{4}$ Assim como as restrições nodais, as forças concentradas fazem sentido na formulação matemática da teoria de vigas. São, na verdade, representações simplificadas de um carga distribuída ao longo de uma superfície de dimensões bastante pequenas se comparadas às demais dimensões do problema.

${ }^{5}$ Tal observação refere-se, naturalmente, ao caso específico estudado de uma viga sub-armada
} 
reduzidas foram incluídos, provocando a distorção angular dos elementos próximos às regiões do apoio e de aplicação da força. Como consequiência, ocorre um empobrecimento da aproximação linear nestas regiões, provocado pelo mapeamento entre as coordenadas naturais e físicas dos elementos distorcidos. Com base nas observações do exemplo da seção 3.2.1, pode-se esperar que o efeito nocivo de tal mapeamento seja anulado com o enriquecimento realizado nas nuvens que contêm esses elementos;

integração numérica: realizada através da quadratura de Gauss-Legendre. Para a Malha I foram utilizados $2 \times 2$ pontos por elemento. Já para para a Malha II, foi utilizado um conjunto de $4 \times 4$ pontos para os elementos próximos do apoio e da força $q$. Para os demais elementos, de dimensões maiores, foram empregados $6 \times 6$ pontos de integração para melhor representar a distribuição do dano. Este, aliás, é um número superior ao exigido por qualquer uma das aproximações definidas na Malha II. Deve-se lembrar que a evolução do dano faz com que os termos a serem integrados na matriz de rigidez deixem de ser polinomiais e, por isso, não há um número preciso de pontos que permita realizar a integração exatamente. Considerando-se esse fato, foram realizados vários experimentos com diferentes ordens de integração. Concluiu-se que, para a solução procurada, a ordem acima descrita permite uma boa aproximação aliada a um menor esforço computacional;

análise não-local: foi adotado um raio de $3 \mathrm{~cm}$ para a análise não-local. Análises realizadas com $r_{\mathrm{nl}}=0 \mathrm{~cm}$ e $r_{\mathrm{nl}}=1,5 \mathrm{~cm}$ produziram resultados bastante instáveis no intervalo de carregamento de 12 a $14 \mathrm{kN}$. Para esses níveis de força concentrada, ocorre uma tendência de concentração do dano, correspondente ao aparecimento de fissuras na região de momento positivo no meio do vão. A utilização de $r_{\mathrm{nl}}=3 \mathrm{~cm}$ tem um efeito regularizador maior, fazendo com que o dano se espalhe, permitindo com que o modelo continue sendo utilizado para reproduzir a resposta global da estrutura;

não-linearidade física: como já comentado anteriormente, o comportamento do concreto foi simulado através do Modelo de Mazars. Os parâmetros utilizados foram na maioria os mesmos empregados para a análise uni-axial através do Método das Nuvens $h p$, tabelas 5.1. No lugar de $A_{T}=0,995$, contudo, adotou-se $A_{T}=0,7$. Este é um artifício válido, empregado para retardar uma evolução 
muito acelerada do dano associada à descrição do ramo do amolecimento que seria obtida com $A_{T}=0,995$, gráfico da Figura 5.7. Um efeito equivalente poderia ser obtido aumentando-se ainda mais o raio para a análise não-local. Nesse caso, porém, existiria a possibilidade de se ter uma resposta muito mais rígida do que a observada experimentalmente. Em PEREGO (1989), o tema da identificação paramétrica com o modelo de Mazars é discutido em detalhes;

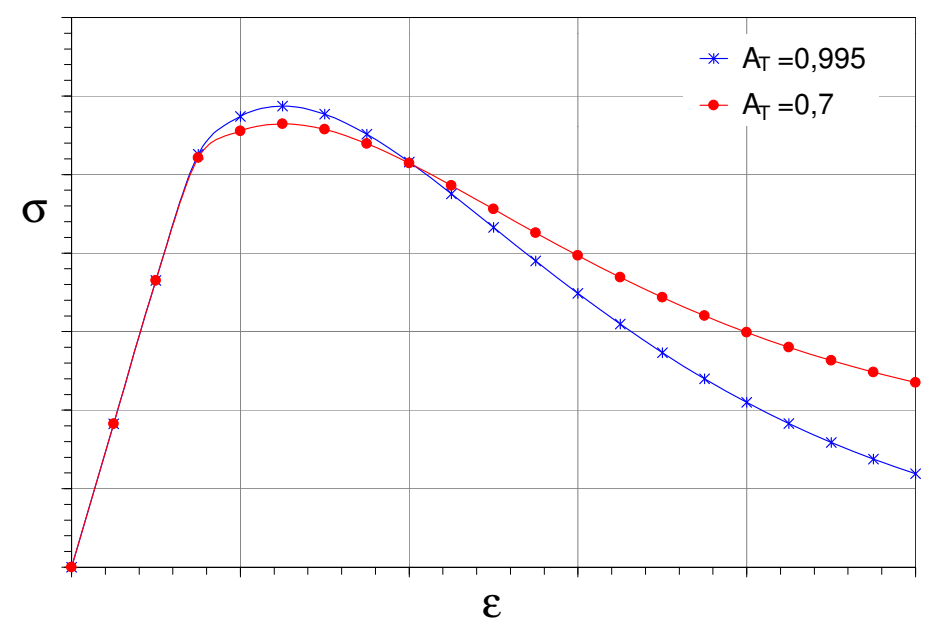

Figura 5.7: Curvas de $\sigma \times \varepsilon$ variando-se $A_{T}$

equilíbrio: o Método de Newton-Raphson foi empregado para a solução do problema não-linear físico. Como critério de convergência do algoritmo de solução iterativa, adotou-se a norma de energia incremental com tolerância de $0,5 \%$ com relação à norma obtida para primeiro passo elástico. Deve-se salientar que o emprego de uma tolerância inferior a $0,5 \%$ consiste em uma condição muito forte para a convergência da análise realizada com a Malha I, especialmente para o intervalo de força entre 12 e $14 \mathrm{kN}$. O mesmo não ocorre para a Malha II pois, como será observado posteriormente, o enriquecimento polinomial suaviza a distribuição do dano produzindo uma resposta global mais estável. Os primeiros 4 passos incrementais do carregamento representaram, cada um, $9 \%$ da força total aplicada $(30 \mathrm{kN})$, sendo seguidos por um passo com $4 \%, 26$ passos com $1 \%$ e os 17 últimos passos com $2 \%$. Esta forma adotada para aplicação do carregamento se deve à elevada não-linearidade observada a partir de 12 kN;

montagem da matriz de rigidez: na montagem da matriz de rigidez adotou-se a formulação secante. Para evitar o mal-condicionamento da matriz de rigidez, para 
efeitos de sua atualização o dano foi limitado $(D \leq 0,87)$;

procedimento para a solução do sistema de equações: na análise feita com a Malha I foi utilizado o método direto de eliminação de Gauss com pivotação parcial. Já para a análise com a Malha II, empregou-se o procedimento de Babuškca, apêndice $\mathrm{C}$, com tolerância de $10^{-10}$ e pertubação da diagonal principal $\epsilon=$ $10^{-8}$. Valores inferiores de $\epsilon$ bem como o emprego da rotina MA27, HSL (2000), produziram resultados inconsistentes. Um explicação para esse fato consiste nos altos níveis locais de danificação que podem elevar o número de condição da matriz de rigidez (mesmo com a limitação do dano a 0,87). É, portanto, um problema relacionado com o modelo constitutivo adotado;

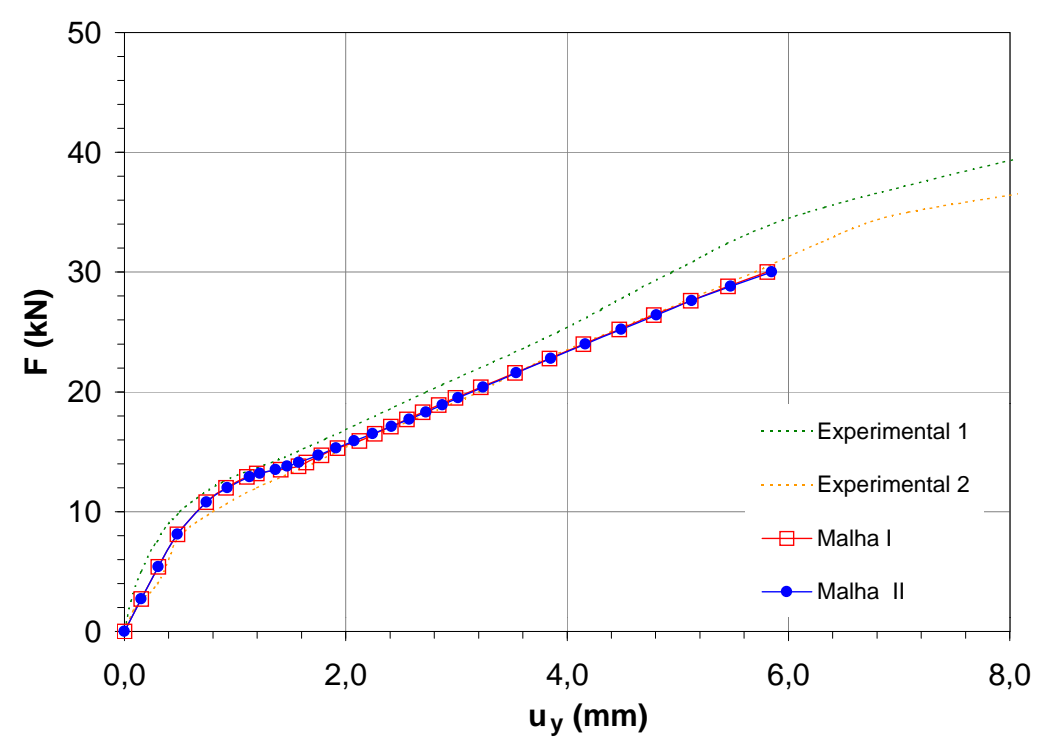

Figura 5.8: Análise estática

Os resultados experimentais de ÁLVARES (1993) são confrontados com os resultados das análises numéricas no gráfico da Figura 5.8. As curvas descrevem a história do carregamento $F$, (equivalente à resultante da carga distribuída $q$ ) relacionando-o ao deslocamento vertical $u_{y}$ (medido no meio do vão, na face inferior da viga). Pode-se afirmar que as respostas numéricas globais da estrutura são, praticamente, coincidentes para as duas análises com as malhas I e II, aproximando-se satisfatoriamente dos resultados experimentais. A partir do nível de força de $30 \mathrm{kN}$ a convergência torna-se mais lenta, mesmo para incrementos de força bastante pequenos contribuindo, por isso, a adoção da limitação do dano para fins de avaliação de matriz de rigidez global. Além 
disso, é acima de $30 \mathrm{kN}$ que se observa a plastificação da armadura, não considerada para a análise, e a resposta numérica deixa de aproximar o resultado experimental. Por estas razões, optou-se por interromper a análise nesse ponto.

As Figuras 5.9(a) e 5.9(b) representam os mapas de distribuição do dano para o nível de força $F=14,4 \mathrm{kN}$, correspondentes ao $13^{\circ}$ passo das análises com as malhas I e II respectivamente. Nota-se que a região de distribuição do dano, obtida com as duas malhas, é bem semelhante. Na Figura 5.9(a), porém, ocorre uma maior concentração, o que pode ser explicado pelo elevado nível de discretização da Malha I se comparado ao da Malha II. Testes realizados com aproximações polinomiais de ordem inferior e superior à apresentada na Figura 5.6(b) indicaram que à medida que se eleva a ordem polinomial da aproximação, mais próxima a distribuição do dano fica do observado na Figura 5.9(a).

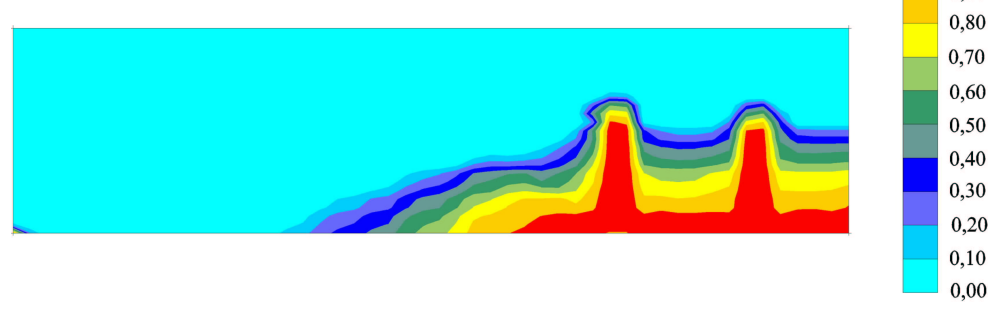

(a) Análise através da Malha I - $F=14,4 \mathrm{kN}$

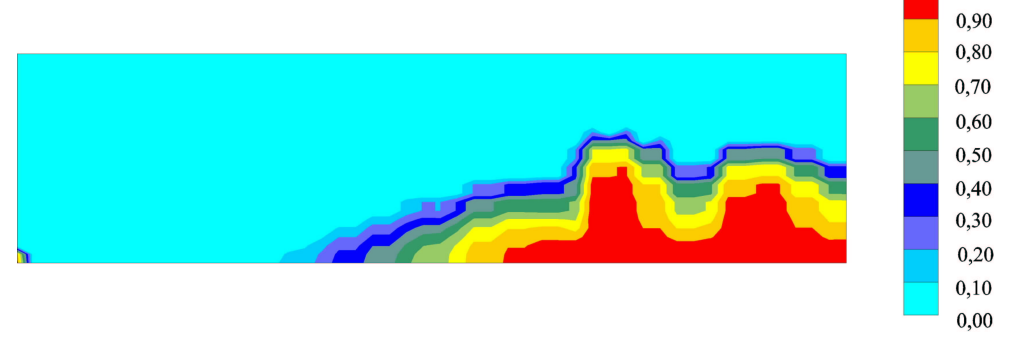

(b) Análise através da Malha II - $F=14,4 \mathrm{kN}$

Figura 5.9: Mapa da distribuição do dano

Com base nesses resultados, averigua-se a viabilidade de se usar um malha grosseira enriquecida polinomialmente em problemas cujo comportamento não-linear decorre da evolução do dano. Basta, para isso, que se utilize uma estratégia eficiente para considerar a armadura e que a aproximação não seja prejudicada pela presença de elementos distorcidos, necessários para a introdução das condições de contorno. $\mathrm{O}$ primeiro quesito é verificado através da técnica de embutimento do aço de RANJBA- 
RAN (1991). Com relação à distorção dos elementos, essa não interfere na qualidade de aproximação desde que a nuvem de elementos distorcidos seja enriquecida polinomialmente pela estratégia empregada para o MEFG, por exemplo.

Naturalmente, respostas semelhantes poderiam ser obtidas com o MEF, utilizandose elementos de ordem polinomial superior. Uma das opções consiste na utilização dos elementos Lagrangianos. Tal procedimento, entretanto, introduziria, para a malha utilizada, um número total de graus de liberdade superior ao do MEFG, como já discutido na seção 3.2.1. Além disso, tal formulação não é adequada à introdução de estratégias de refinamento adaptativo. Por outro lado, pode-se usar a versão hierárquica do MEF, SZABÓ; BABUŠKA (1991) e DEMKOWICZ (1989). Pelas mesmas razões apresentadas para a formulação Lagrangianas, com o MEF hierárquico o número de graus de liberdade é superior ao do MEFG para uma malha bastante discretizada. Soma-se a esse fato, a facilidade de implementação da estratégia de enriquecimento do MEFG, sem a necessidade de se compatibilizar as aproximações de elementos vizinhos com ordem polinomial diferente.

Comprovada a viabilidade de se usar o MEFG para a análise de problemas semelhantes ao apresentado, resta, ainda, estabelecer a melhor maneira de se definir o nível de enriquecimento exigido para a solução que se queira encontrar. A solução encontrada com a malha da Figura 5.6(b) representou bem o comportamento global da estrutura. Não há, todavia, como saber, a menos da realização de novas análises, se tal aproximação é a ideal, ou seja, aquela que melhor aproxima o problema para uma menor quantidade de graus de liberdade. Para responder esta questão é necessário que se estabeleça, primeiramente, um critério de erro. Com essa informação, torna-se possível introduzir uma abordagem adaptativa, em que a qualidade da solução é testada automaticamente e, caso seja necessário, uma nova aproximação é construída. É esse o assunto do próximo capítulo em que um estimador de erro e um algoritmo adaptativo são descritos para o contexto do MEFG.

\subsection{Chapa de Concreto Tracionada}

Como última experimentação numérica deste capítulo utiliza-se um problema bastante simples, um chapa de concreto tracionada, para avaliar a capacidade da aproximação enriquecida polinomialmente de representar o fenômeno de localização do dano. 
Na Figura 5.10 estão representadas a geometria e as condições de contorno da chapa de concreto com $10 \mathrm{~cm}$ de espessura . O vínculo no ponto $B$, correspondente ao deslocamento vertical, é inserido, simplesmente, para eliminar movimentos de corpo rígido. A solicitação de tração da estrutura é definida impondo-se o afastamento, na direção horizontal, das faces $A B$ e $C D$. Os deslocamentos correspondentes a um afastamento de $6 \times 10^{-2} \mathrm{~mm}$ são impostos de forma incremental possibilitando a análise não-linear pelo método de Newton-Raphson. O modelo constitutivo é o de Mazars com abordagem não-local, para o qual foram adotados os seguintes parâmetros:

$$
\begin{array}{cccc}
A_{T}=0,7 & B_{T}=8000 \quad A_{C}=0,85 & B_{C}=1050 \\
\varepsilon_{d 0}=0,0001 & E=29200 \mathrm{MPa} & \nu=0,2 &
\end{array}
$$

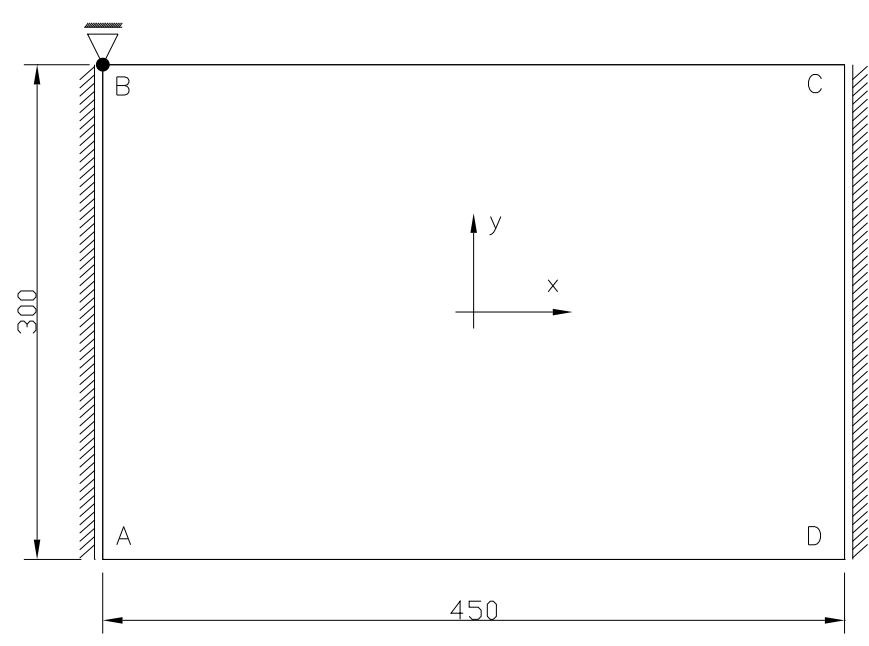

Figura 5.10: Chapa de concreto - geometria e condições de contorno - medidas em mm

Dois tipos de malha foram utilizadas. A malha da Figura 5.11(a) é formada por 600 elementos quadrangulares de 4 nós, com interpolação linear, sendo utilizada na análise convencional pelo MEF. As análises feitas com o MEFG empregam a malha da Figura 5.11(b). O enriquecimento da aproximação, expressões (3.19) e (3.20), é realizado de acordo com os índices $p_{1}$ e $p_{2}$ associados a cada nó e correspondentes à ordem máxima do polinômio que se pode reproduzir com as funções de forma desse nó. Foram testadas cinco discretizações, variando-se $p_{2}=1, \cdots, 5$ e mantendo-se $p_{1}=1$ constante.

Para as análises em estado plano de tração foram admitidos:

montagem da matriz de rigidez: da mesma forma que no exemplo da viga de concreto armado, adotou-se a formulação secante e o dano limitado a 0,87 na construção da matriz de rigidez; 


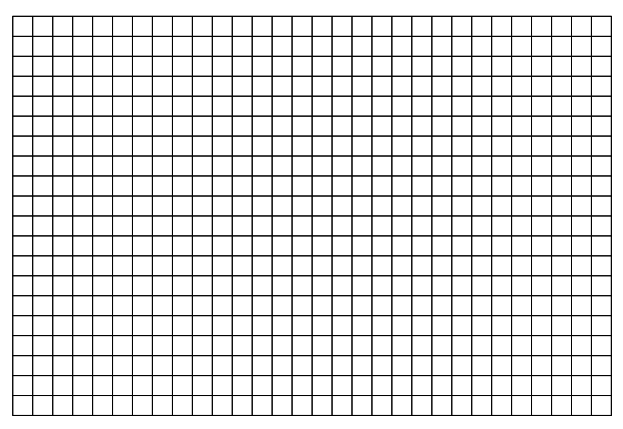

(a) Malha com 600 elementos, aproximação linear

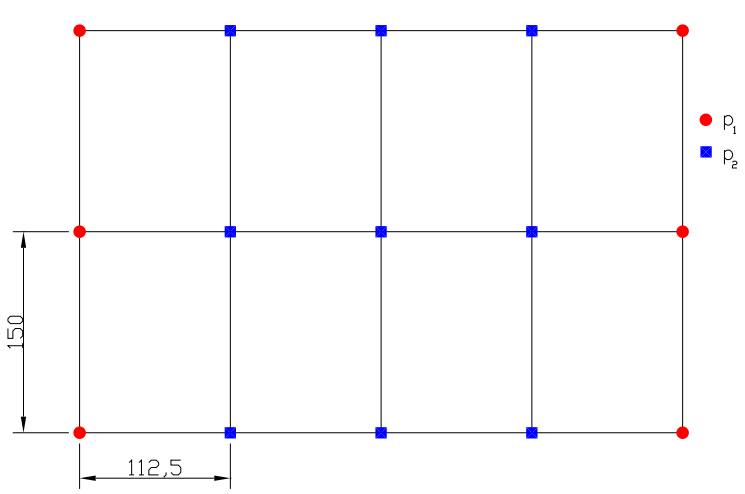

(b) Malha com enriquecimento polinomial

Figura 5.11: Malhas utilizadas para a análise - medidas em mm

solução do sistema de equações: na análise com a malha da Figura 5.11(a) utilizase o método direto de eliminação de Gauss com pivotação parcial. Nas demais análise, o procedimento de Babuška foi adotado com tolerância de $1 \times 10^{-10} \mathrm{e}$ perturbação da diagonal principal $\epsilon=1 \times 10^{-8}$;

análise não-local: o raio adotado da análise não-local vale $r_{\mathrm{nl}}=1,5 \mathrm{~cm}$. Este valor foi escolhido para que coincidisse com largura da faixa de localização do dano;

localização do dano: para provocar a localização do dano optou-se por reduzir o parâmetro $\varepsilon_{d 0}$ para 0,000 095 nos pontos de Gauss localizados entre 210 e $240 \mathrm{~mm}$ da face $A B$. Na malha da Figura 5.11(a) esses pontos pertencem às duas colunas centrais de elementos;

integração numérica: foi adotada a quadratura de Gauss-Legendre. Para a malha da Figura 5.11(a) foram utilizados $2 \times 2$ pontos de Gauss. Por sua vez, na malha da Figura 5.11(b), foram empregados $8 \times 8$ pontos de integração nas duas colunas centrais de elementos e nos demais $2 \times 2$ pontos. Dessa forma, consegue-se ter na região de localização do dano um número equivalente de pontos segundo a direção $x$;

equilíbrio: para a aplicação do Método de Newton-Raphson, utilizou-se no critério de convergência em energia, uma tolerância relativa de 0,1\% com relação ao primeiro passo elástico. Os deslocamentos impostos foram divididos ao longo de 13 passos, dos quais o primeiro corresponde a $70 \%$ do valor final e cada um dos demais passos representam um acréscimo de 2,5\%. 


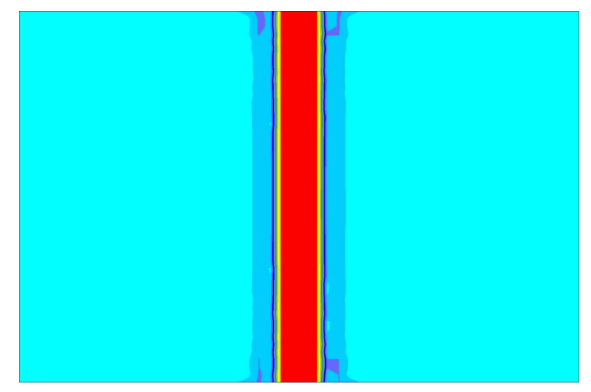

(a) Malha com 600 elementos
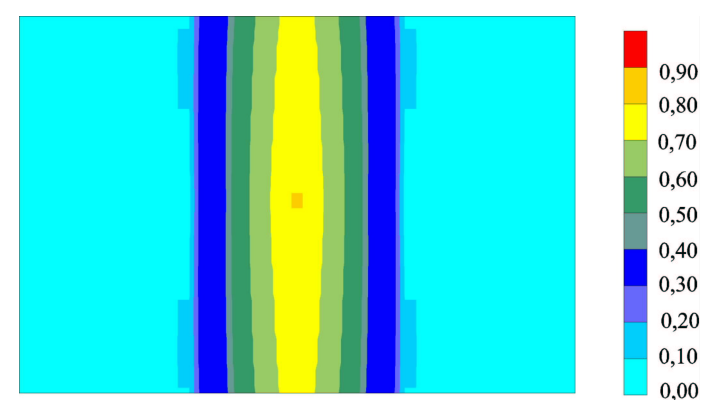

(c) Malha com 8 elementos, $p_{1}=1 p_{2}=2$
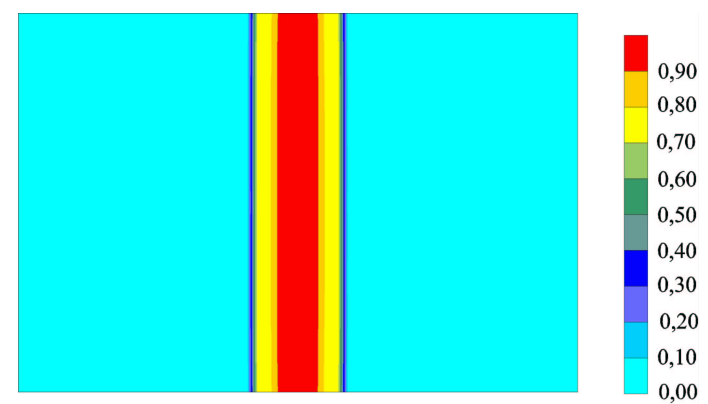

(e) Malha com 8 elementos, $p_{1}=1 p_{2}=4$

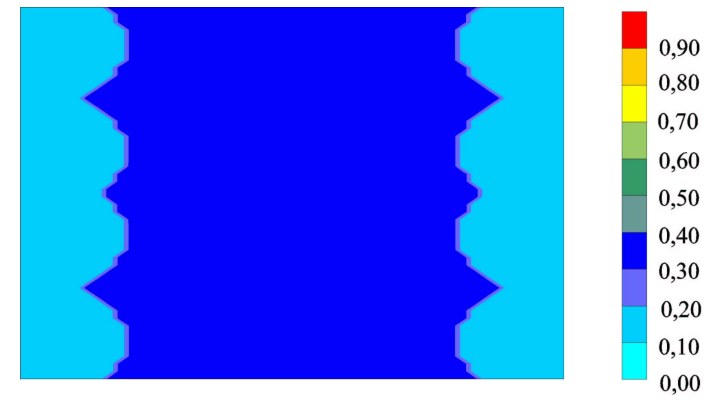

(b) Malha com 8 elementos, $p_{1}=1 p_{2}=1$

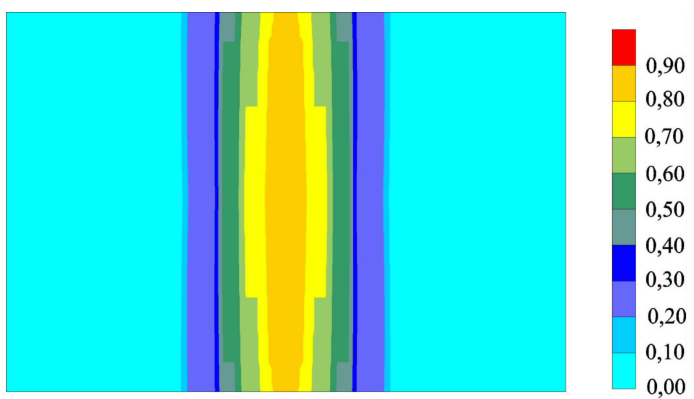

(d) Malha com 8 elementos, $p_{1}=1 p_{2}=3$

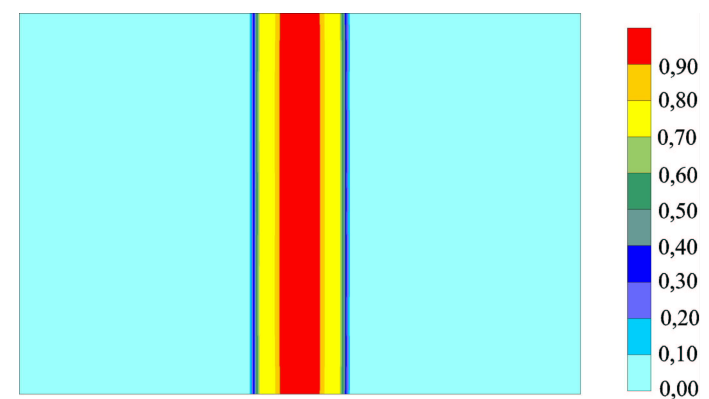

(f) Malha com 8 elementos, $p_{1}=1 p_{2}=5$

Figura 5.12: Mapa da distribuição do dano 
Na Figura 5.12 encontram-se representadas as distribuições do dano, para cada uma das 6 análises, ao final da processo incremental de deslocamento. Observa-se, à medida que se aumenta $p_{2}$, para as análises com a malha de 8 elementos, uma convergência da faixa de localização do dano para a largura de $3 \mathrm{~cm}$, encontrada com a malha de 600 elementos. Uma pequena diferença, contudo, entre os resultados da análise do MEF e do MEFG com $p_{2}=5$ merece ser salientada, podendo ser melhor observada na Figura 5.13. Enquanto que na distribuição de dano obtida para a malha de 600 elementos a transição entre os valores máximos e mínimo é brusca, o mesmo não ocorre na malha com enriquecimento polinomial. Explica-se esse fato pela suavização da aproximação induzida pelo emprego do refinamento polinomial. Ainda assim, pode-se dizer que o fenômeno de concentração do dano pôde ser bem representado com MEFG através da técnica de enriquecimento da PU.

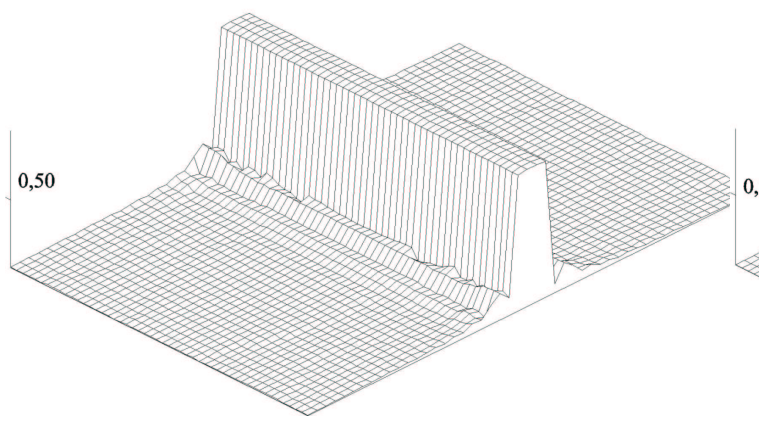

(a) Malha com 600 elementos, aproximação linear

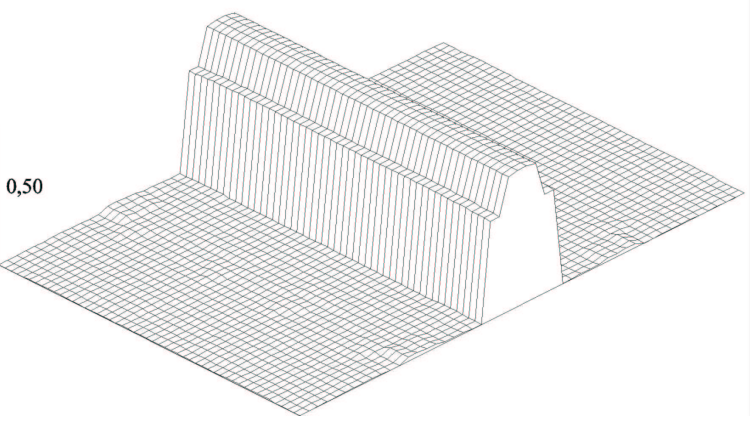

(b) Malha com 8 elementos, $p_{1}=1 p_{2}=5$

Figura 5.13: Superfície representativa do dano

Também com o MEF seria possível utilizar funções de grau polinomial mais elevado. Estas, porém, exigiriam uma compatibilização das aproximações entre elementos caso o grau do polinômio variasse como neste exemplo. Esta preocupação não existe quando a análise é realizada com o MEFG, conforme discutiu-se na seção 2.5.1. 


\section{Capítulo 6}

\section{Estimador de Erro}

No exemplo da seção 5.3.1, foram necessários poucos elementos para aproximar a resposta não-linear de uma viga de concreto armado. Isto somente foi possível graças ao enriquecimento polinomial não uniforme realizado sobre as funções da PU e que se concentrou na região de maior evolução do dano. A escolha de como e quanto enriquecer cada nuvem foi resultado de uma sequiência de enriquecimentos definidos de maneira intuitiva e testados para o problema. Utilizou-se, portanto, de um processo meramente comparativo, não havendo garantias de se ter chegado a uma descrição "ótima”, ou seja, aquela que, para um dado número de graus de liberdade, permite obter o melhor resultado possível. Com esse intuito, porém, torna-se necessário o emprego de medidas de erro que forneçam informações sobre a qualidade da solução aproximada e em quais regiões ela precisa ser melhorada. Em uma abordagem adaptativa, tais informações podem ser usadas para modificar automaticamente o enriquecimento da PU, otimizando o processo de construção da aproximação.

No MEFG, a maneira como enriquecimento é realizado faz com que a introdução de uma estratégia adaptativa torne-se bastante atraente. O sucesso desse empreendimento depende, entretanto, da medida de erro adotada. Neste capítulo, são discutidos uma medida de erro e os algoritmos adaptativos utilizados para as análises com o MEFG, sejam elas lineares ou não-lineares, estas últimas governadas pelo fenômeno de danificação. A medida utilizada para avaliar o erro da aproximação é obtida do Método dos Resíduos em Elementos Equilibrados, adaptado à formulação do MEFG. Antes, porém, são apresentados alguns conceitos essenciais, juntamente com uma pequena revisão das pesquisas no tema erro e adaptatividade. Com essa revisão, não se tem a pretensão de exaurir o assunto mas, apenas, apresentar o seu estágio atual de desenvolvimento no qual este trabalho se insere. 


\subsection{Conceitos e Definições para o Estudo de Erro}

A seguir, são apresentados algumas definições e conceitos comumente usados no estudo do erro. Para maiores detalhes, podem ser consultados DUARTE (1991), SZABÓ; BABUŠKA (1991), BABUŠKA (1994a) e BABUŠKA (1994b) por exemplo.

erro: Seja a forma variacional do PVC definida em (3.12) e cuja a solução exata é representada pela função vetorial $\boldsymbol{u}$. Considera-se, também, a correspondente aproximação de Galerkin (3.13) e a respectiva solução $\tilde{\boldsymbol{u}} \stackrel{\text { def }}{=} \boldsymbol{u}_{p} \in X_{p}$. Define-se a função de erro da aproximação $\boldsymbol{u}_{p}$ como:

$$
\boldsymbol{e}_{p} \stackrel{\text { def }}{=} \boldsymbol{u}-\boldsymbol{u}_{p}
$$

Em DUARTE (1991), observa-se que essa função não representa o erro total da solução pois não leva em conta os erros de arredondamento, da integração numérica, da representação do carregamento e da geometria;

condição de ortogonalidade do erro: Sendo $\boldsymbol{v}_{p} \in X_{p} \subset \mathcal{H}^{1}$, a equação (3.12) pode ser reescrita como:

$$
\mathcal{B}\left(\boldsymbol{u}, \boldsymbol{v}_{p}\right)=l\left(\boldsymbol{v}_{p}\right)
$$

Subtraindo-se, então, (3.13) de (6.2) e pelo fato do funcional $\mathcal{B}(\bullet, \bullet)$ ser bilinear, chega-se a seguinte expressão, ODEN; REDDY (1976):

$$
\mathcal{B}\left(\boldsymbol{u}-\boldsymbol{u}_{p}, \boldsymbol{v}_{p}\right)=\mathcal{B}\left(\boldsymbol{e}_{p}, \boldsymbol{v}_{p}\right)=0
$$

que é interpretada como uma condição de ortogonalidade, ou seja, a função erro $\boldsymbol{e}_{p}$ da aproximação de Galerkin pertence ao espaço ortogonal a $X_{p}$ no sentido da métrica definida pelo funcional $\mathcal{B}(\bullet, \bullet)$;

norma energia: medida associada à forma bi-linear $\mathcal{B}(\bullet, \bullet)$ e definida como:

$$
\|\boldsymbol{w}\|_{u} \stackrel{\text { def }}{=} \sqrt{\mathcal{B}(\boldsymbol{w}, \boldsymbol{w})}, \quad \forall \boldsymbol{w} \in \mathcal{H}^{1}
$$

Observa-se que $\|\boldsymbol{w}\|_{\mathcal{U}}^{2}$ corresponde ao dobro da energia de deformação, $\mathcal{U}$, armazenada na estrutura quando sujeita a um campo de deslocamentos $\boldsymbol{w} \in \mathcal{H}^{1}$. No contexto do MEF, a norma energia pode também ser definida para cada elemento finito $\mathcal{K}$ como: 


$$
\|\boldsymbol{w}\|_{u(\mathcal{K})} \stackrel{\text { def }}{=} \sqrt{\mathcal{B}_{\mathcal{K}}(\boldsymbol{w}, \boldsymbol{w})}, \quad \forall \boldsymbol{w} \in \mathcal{H}^{1}(\mathcal{K})
$$

em que $\mathcal{B}_{\mathcal{K}}(\bullet, \bullet)$ corresponde à restrição do operador $\mathcal{B}(\bullet, \bullet)$ ao elemento $\mathcal{K}$. $\mathrm{O}$ valor da norma de energia para todo o domínio pode ser obtido a partir de contribuições calculadas em cada elemento finito, ou seja:

$$
\|\boldsymbol{w}\|_{\mathfrak{u}}^{2} \stackrel{\text { def }}{=} \sum_{\mathcal{K} \in \Omega} \mathcal{B}_{\mathcal{K}}(\boldsymbol{w}, \boldsymbol{w})
$$

norma energia do erro: o erro de aproximação definido pela expressão (6.1) tem como norma energia de (6.4):

$$
\left\|\boldsymbol{e}_{p}\right\|_{u}=\sqrt{\mathcal{B}\left(\boldsymbol{e}_{p}, \boldsymbol{e}_{p}\right)}
$$

Utilizando-se da condição de ortogonalidade (6.3) pode-se mostrar que para problemas lineares:

$$
\left\|\boldsymbol{e}_{p}\right\|_{u}=\sqrt{\mathcal{B}(\boldsymbol{u}, \boldsymbol{u})-\mathcal{B}\left(\boldsymbol{u}_{p}, \boldsymbol{u}_{p}\right)}
$$

estimador de erro global: medida do erro definida em termos de uma conveniente norma. Empregando-se a norma energia, o estimador de erro global, também denominado simplesmente de estimador de erro, é representado por:

$$
\left\|\tilde{\boldsymbol{e}}_{p}\right\|_{u}
$$

O símbolo $\tilde{e}_{p}$ é utilizado no lugar de $\boldsymbol{e}_{p}$ para lembrar que a medida em questão corresponde a uma estimativa da norma do erro da aproximação $\boldsymbol{u}_{p}$, pois não se conhece, em geral, a solução exata $\boldsymbol{u}$. Na seção 6.3, em que o Método dos Resíduos em Elementos, MRE, é apresentado, $\tilde{\boldsymbol{e}}_{p}$ é obtido localmente sendo denominado função indicadora de erro;

indicador de erro: medida representativa do erro local, limitada ao domínio do elemento no contexto do MEF e definida de forma análoga à do estimador global. É denominado indicador pois é empregado para indicar, no processo adaptativo, as regiões em que o refinamento da solução deve ser realizado. No caso do MRE, 
é definido pela norma de energia das funções indicadoras de erro e assume a seguinte representação:

$$
\tilde{\mathcal{E}}_{\mathcal{K}}=\left\|\tilde{\boldsymbol{e}}_{p}\right\|_{\mathcal{K}}
$$

contribuindo para o cálculo do estimador de erro global segundo o somatório (6.6) para cada elemento finito:

$$
\left\|\tilde{\boldsymbol{e}}_{p}\right\|_{\mathfrak{U}}^{2} \stackrel{\text { def }}{=} \sum_{\mathcal{K} \in \Omega} \tilde{\mathcal{E}}_{\mathcal{K}}^{2}
$$

erro relativo global: corresponde à razão entre as normas do erro $\boldsymbol{e}_{p}$ e da solução $\boldsymbol{u}$, calculadas para todo o domínio $\Omega$. Empregando-se a norma energia tem-se:

$$
\mathcal{E}_{\%} \stackrel{\text { def }}{=} \frac{\left\|\boldsymbol{e}_{p}\right\|_{u}}{\|\boldsymbol{u}\|_{u}} \times 100 \%
$$

No caso de se conhecer apenas a estimativa para o erro, representada por $\left\|\tilde{\boldsymbol{e}}_{p}\right\|$, bem como uma solução aproximada $\boldsymbol{u}_{p}$, a seguinte definição é empregada para o erro relativo global estimado:

$$
\tilde{\mathcal{E}}_{\%} \stackrel{\text { def }}{=} \frac{\left\|\tilde{\boldsymbol{e}}_{p}\right\|_{u}}{\sqrt{\left(\left\|\boldsymbol{u}_{p}\right\|_{u}\right)^{2}+\left(\left\|\tilde{\boldsymbol{e}}_{p}\right\|_{u}\right)^{2}}} \times 100 \%
$$

erro relativo local: de forma consistente com a definição global, o erro relativo local representa a razão entre as normas do erro $\boldsymbol{e}_{p}$ e da solução $\boldsymbol{u}$ em um elemento finito $\mathcal{K}$. Para o caso da norma energia define-se:

$$
\mathcal{E}_{\%}^{\mathcal{K}} \stackrel{\text { def }}{=} \frac{\left\|\boldsymbol{e}_{p}\right\|_{u(\mathcal{K})}}{\|\boldsymbol{u}\|_{u(\mathcal{K})}} \times 100 \%
$$

Já o erro relativo estimado local é definido como:

$$
\tilde{\mathcal{E}}_{\%}^{\mathcal{K}} \stackrel{\left\|\tilde{\operatorname{def}}_{p}\right\|_{u(\mathcal{K})}}{\sqrt{\left(\left\|\boldsymbol{u}_{p}\right\|_{\mathcal{U}(\mathcal{K})}\right)^{2}+\left(\left\|\tilde{\boldsymbol{e}}_{p}\right\|_{u(\mathcal{K})}\right)^{2}}} \times 100 \%
$$

índice de efetividade: medida empregada para aferir a qualidade do estimador de 
erro, sendo definido, globalmente, como:

$$
\theta \stackrel{\text { def }}{=} \frac{\left\|\tilde{\boldsymbol{e}}_{p}\right\| u}{\left\|\boldsymbol{e}_{p}\right\| u}
$$

Pode-se também calcular o índice de efetividade para cada elemento $\mathcal{K}$ como:

$$
\theta_{\mathcal{K}} \stackrel{\text { def }}{=} \frac{\left\|\tilde{\boldsymbol{e}}_{p}\right\|_{u(\mathcal{K})}}{\left\|\boldsymbol{e}_{p}\right\|_{(\mathcal{K})}}
$$

O índice de efetividade somente pode ser obtido para problemas de soluções conhecidas. O ideal, segundo SZABÓ; BABUŠKA (1991) é que se tenha $0,8 \leq$ $\theta \leq 1,2$ e que $\theta \rightarrow 1$ quando $\left\|\boldsymbol{e}_{p}\right\| \rightarrow 0$. Nesse caso, o estimador é denominado exato assintoticamente.

\subsection{Estimadores de Erro e Adaptatividade}

$\mathrm{O}$ estudo de erro e de procedimentos adaptativos corresponde a um capítulo à parte no estudo dos Métodos Numéricos. Basicamente, define-se como adaptativo, qualquer processo que se utilize de resultados intermediários, gerados durante a solução, para aprimorar, de modo ótimo, a resposta numérica. Várias são as estratégias adaptativas utilizadas, em particular no MEF, e que podem ser estendidas aos métodos numéricos em geral. Em todas elas, adotam-se, isoladamente ou combinados entre si, os seguintes processos:

processo $h$ : os elementos da malha são subdivididos em elementos menores, simultaneamente ou não. Tal processo corresponde, no Método das Nuvens $h p$ e no MGLE, à introdução de novos nós sobre o conjunto existente no domínio. No MEFG não há diferenças com relação ao MEF;

processo $p$ : caracteriza-se pelo aumento no grau polinomial da aproximação em um conjunto de elementos, ou mesmo em toda a malha. No MGLE, isso somente é obtido com a introdução de novos monômios de ordem superior na base de funções $\boldsymbol{P}$ responsável pela geração das funções originais. No Método das Nuvens $h p$ e no MEFG o enriquecimento polinomial é vinculado às nuvens e não mais aos elementos, sendo obtido pela multiplicação das funções PU por monômios; 
processo $r$ : os nós são redistribuídos ao longo do domínio. Tal definição é válida também para o MGLE, para o Método das Nuvens e para o MEFG.

Pode-se ainda destacar o processo $s$, proposto por FISH (1992) e FISH (1994), em que novos elementos são sobrepostos à malha original, enriquecendo-a de maneira hierárquica.

Por outro lado na seção 3.2.4, foi mostrada, por meio de um exemplo numérico, a possibilidade de se melhorar a aproximação do MEFG através da multiplicação da PU por funções não polinomiais. Abre-se, assim, a possibilidade de se ter um novo tipo de processo adaptativo, em que a aproximação é enriquecida por funções "especiais". Para isto, é necessário que se tenha um banco de dados contendo tais funções, bem como um critério vinculado, por exemplo, ao tipo de geometria, carregamento e propriedades do material, que podem mudar durante a análise dependendo do tipo de problema. Uma interessante discussão a esse respeito pode ser encontrada em STROUBOULIS; BABUŠKA; COPPS (2000) e, da mesma forma, em STROUBOULIS; COPPS; BABUŠKA (2001).

Nas estratégias adaptativas, procura-se garantir, minimamente, a qualidade da aproximação, mediante alguma medida de erro global. Quando essa medida é baseada em informações extraídas da própria solução aproximada tem-se o estimador de erro a posteriori. Uma medida bastante usada, especialmente para problemas elípticos, é a estimativa da norma energia do erro global, representada em (6.9) por $\left\|\tilde{\boldsymbol{e}}_{p}\right\|$ . Por sua vez, o refinamento da aproximação através de um dos processos adaptativos descritos anteriormente, é deflagrado por medidas de erro locais, denominadas indicadores de erro. Estas medidas indicam as regiões do domínio em que a discretização deve ser melhorada. Como idéia básica adota-se, geralmente, o princípio da eqüidistribuição de erro, discutido para o MEF em BABUŠKA; RHEINBOLDT (1979). Segundo esse princípio, a aproximação é considerada "ótima" caso o erro medido na norma energia seja constante em todos elementos do domínio. Dessa forma, a aproximação é refinada nas regiões em que os indicadores de erro fornecerem valores bem superiores aos encontrados no restante do domínio. Em SZABÓ; BABUŠKA (1991), afirma-se que, mesmo apresentando um índice de efetividade pobre, expressão (6.17), os indicadores podem ser utilizados com sucesso no processo adaptativo. Outro papel, muitas vezes desempenhado pelos indicadores, é na construção do estimador de erro global. No MRE, por exemplo, já citado na seção 6.1, o estimador global é obtido pela expressão (6.11), através do somatório dos indicadores calculados em cada elemento finito. 
No âmbito mais geral, abrangendo outros métodos numéricos, vários são os procedimentos difundidos na literatura para se ter um eficiente estimador. A maioria desses procedimentos podem ser reunidos em dois grandes grupos, conforme a técnica usada seja baseada em:

estimativa do resíduo: o estimador é construído a partir do resíduo da solução aproximada e mede o quanto a solução obtida falha em satisfazer as equações diferenciais e as condições de contorno que governam o PVC;

pós-processamento dos gradientes da solução: sua idéia principal, bastante simples, consiste em utilizar a diferença entre o gradiente da solução aproximada e uma função recuperada de um pós-processamento dessa solução. A técnica de obtenção destas funções recuperadas, ou suavizadas, como muitas vezes são denominadas, varia entre as diversas versões presentes nessa categoria de estimadores.

Além dos estimadores reunidos nestas duas categorias, outros poderiam ser citados, tais como os métodos duais, métodos de interpolação e de análise assintótica.

\subsubsection{Estimadores de Erro no MEF}

Estimativas baseadas no resíduo da solução estão entre as primeiras técnicas a considerar o erro de aproximação do MEF. Fundamentada nos trabalhos pioneiros de Babuška e colaboradores, entre eles BABUŠKA; RHEINBOLDT (1978a), (1978b), (1979), essa estratégia foi desenvolvida inicialmente na versão explícita em que as estimativas de erro são calculadas diretamente das normas das funções de resíduo. Por sua vez, a versão implícita do método teve sua formulação introduzida em trabalhos como DEMKOWICZ; ODEN; STROUBOULIS (1984) e BANK; WEISER (1985). Nessa abordagem, as funções determinadas pelo resíduo da solução são empregadas como dados para PVC associados aos erros locais. A solução de cada um desses problemas define as funções de erro empregadas para se construir o estimador de erro global e também utilizadas como indicadores do procedimento adaptativo. Quando tais indicadores são definidos em sub-regiões ou parcelas do domínio aproximado, tem-se o Método dos Resíduos em Sub-domínios (MRS), BABUŠKA; RHEINBOLDT (1978a). Por outro lado, quando o problema do erro é formulado para cada elemento isoladamente define-se o Método dos Resíduos em Elementos (MRE). Um importante estudo a esse respeito pode ser encontrado em ODEN (1989). 
Mais recentes que os métodos baseados no resíduo da aproximação, os estimadores obtidos do pós-processamento do gradiente da solução foram introduzidos em ZIENKIEWICZ; ZHU (1987). Nesse trabalho, uma função contínua global é obtida a partir da projeção dos gradientes da solução no espaço das funções de interpolação do MEF. A técnica de projeção é do tipo $L_{2}$, usada segundo ODEN; BRAUCHLI (1971). Em ZIENKIEWICZ; ZHU (1992a) e ZIENKIEWICZ; ZHU (1992b) as funções suavizadas passam a ser determinadas localmente por meio de um ajuste por mínimos quadrados dos valores obtidos em pontos do domínio denominados super-convergentes ${ }^{1}$. Em razão da dificuldade de se obter tais pontos para diferentes tipos de aproximação, é proposto o Método de Recuperação pelo Equilíbrio das Parcelas (Recovery by Equilibrium of Pathches), MREP, BOROOMAND; ZIENKIEWICZ (1997). Diversos outros trabalhos, envolvendo estimadores baseados no pós-processamento do gradiente da solução, podem ser, ainda, citados tais como BLACKER; BELYTSCHKO (1994), CARSTENSEN; FUNKEN (2000a) e CARSTENSEN; FUNKEN (2000b).

Mais detalhes sobre os estimadores de erro no MEF podem ser encontrados por exemplo em ODEN (1989), DUARTE (1991) e STROUBOULIS; HAQUE (1992). Recomenda-se, também, a leitura da monografia de AINSWORTH; ODEN (1997), onde uma vasta bibliografia é listada a esse respeito. Segundo os autores, a estrutura formal do MEF adaptativo para problemas elípticos lineares está, atualmente, bem fundamentada, tendo alcançado sua maioridade. Diversas pesquisas, como em BABUŠKA (1994a) e BABUŠKA (1994b), têm sido realizadas no intuito, apenas, de se averiguar limites e eficiência dos diversos estimadores de erro existentes. Nota-se, contudo, que o mesmo estágio de desenvolvimento não é observado para problemas não-lineares e os estudos voltados para tais aplicações encontram-se, ainda, em franco desenvolvimento.

O trabalho de RHEINBOLDT (1985) talvez seja um dos primeiros a utilizar a teoria de estimadores de erro, no caso formulações baseadas no resíduo da solução, em problemas com não-linearidade física. Também nessa linha, encontram-se experimentações realizadas em RAMM; CIRAK (1997) para problemas de estruturas de paredes finas com não-linearidade física ou geométrica.

Ainda em RAMM; CIRAK (1997) e particularmente CIRAK; RAMM (2000),

\footnotetext{
${ }^{1}$ Os pontos super-convergentes são posições do domínio em que se pode esperar que o gradiente da aproximação tem melhor taxa de convergência do que a própria solução aproximada, AINSWORTH; ODEN (1997). Tais pontos existem em condições muito especiais, e um extenso debate, ainda hoje, é travado a esse respeito como se verifica, por exemplo, em HILLER; BATHE (2001).
} 
a partir do resíduo da solução e aplicando-se o teorema da reciprocidade de Betti e Rayleigh, obtém-se um problema dual para a estimativa de erro em pontos de equilíbrio do PVC não-linear. O carregamento para tal problema é determinado em analogia ao conceito de linhas (ou superfícies) de influência, relacionando o erro de aproximação em todo o domínio com uma variável considerada isoladamente. Campos de tensões e deformações suavizadas são empregados em CIRAK; RAMM (2000) para calcular o resíduo da solução.

Outro estimador baseado em técnicas duais pode ser encontrado no trabalho de RANNACHER; SUTTMEIER (1999), proposto a partir da abordagem geral desenvolvida em JOHNSON; HANSBO (1992). Uma forma dual global e linearizada para o PVC não-linear é estabelecida para o funcional representativo de uma quantidade física de interesse, pontual ou não. A solução desse novo problema é utilizada como função de ponderação dos resíduos locais do PVC, definindo um estimador de erro ponderado para a quantidade física selecionada.

Nas abordagens baseadas no pós-processamento dos gradientes da solução podem ser citados os trabalhos de BOROOMAND; ZIENKIEWICZ (1999) bem como CARSTENSEN; ALBERTY (2000), ambos para problemas elasto-plásticos. No primeiro, emprega-se o MREP para a condução do processo $h$-adaptativo em cada passo do processo de solução incremental. Por sua vez, CARSTENSEN; ALBERTY (2000) é um artigo de cunho teórico em que se procura mostrar a confiabilidade da classe de estimadores desenvolvida por Zienkiewicz e Zhu.

Em GALLIMARD; LADEVÈZE; PELLE (1996) e, posteriormente complementada em GALLIMARD; LADEVÈZE; PELLE (2000) é proposta uma medida de erro global associada à relação constitutiva e capaz de abranger não apenas o erro de aproximação da malha, como também o erro devido à discretização no tempo, introduzido pelo método incremental de solução do problema não-linear físico. Utiliza-se para isso do postulado de estabilidade de Drucker, CHEN; HAN (1988), e de técnicas para a construção de campos de tensões admissíveis.

Problemas com localização de deformação apresentam uma dificuldade a mais com relação aos demais problemas com não-linearidade física: a perda da elipsidade das equações de governo. Como os métodos baseados no resíduo da solução são formulados para problemas elípticos, não podem ser aplicados nessa situação. Em ORTIZ; QUIGLEY, IV (1991), entretanto, define-se uma estratégia adaptativa na qual procura-se refinar (tipo $h$ ) os elementos em que a variação da solução é observada den- 
tro de uma certa tolerância prescrita. Também aplicado a problemas de localização, destaca-se o trabalho de PERIĆ; YU; OWEN (1994), onde é apresentado um estimador baseado na estratégia de suavização da solução de ZIENKIEWICZ; ZHU (1987), adequadamente modificada para considerar o fenômeno estudado.

Existem, naturalmente, diversos outros estudos para o desenvolvimento de estimadores e estratégias adaptativas em problemas não-lineares. Os artigos citados são, apenas, uma amostra do vasto campo de pesquisa existente. Juntamente com as informações apresentadas na próxima seção, esta pequena revisão é, contudo, suficiente para situar o leitor em que contexto a implementação de uma formulação adaptativa para o MEFG é introduzida.

\subsubsection{Estimadores de Erro no MEFG}

O emprego de estimadores de erro juntamente com o MEFG é um campo ainda pouco explorado. Explica-se esse fato mais pelo caráter recente do método do que por "possíveis" diferenças em relação ao MEF.

Em DUARTE; ODEN (1996a), ainda na formulação do Método das Nuvens $h p$, uma abordagem semelhante ao do método dos resíduos na versão explícita é empregada para a solução de um problema de Poisson em $\mathbb{R}^{2}$. O erro estimado é utilizado na condução de procedimentos adaptativos do tipo $h, p$ e $h p$. No assim chamado MEFPU, uma discussão teórica a esse respeito é conduzida, mas em um contexto em que a PU é construída de forma genérica, seja pelo MMQM ou através de polinômios como no MEF, BABUŠKA; MELENK (1997). Em ambos os trabalhos, o indicador de erro é calculado em cada nuvem e não mais em elementos. Para isso, considera-se que as funções de aproximação sejam regulares o suficiente para que as derivadas da solução aproximada sejam contínuas ao longo de todo o domínio. Evita-se, assim, que saltos nas derivadas tenham de ser calculados no interior das nuvens e no seu contorno.

Para o MEFG, propriamente dito, podem ser citados dois trabalhos em que os estimadores de erro são discutidos, primeiramente em BABUŠKA (1997) e mais tarde em STROUBOULIS; COPPS; BABUŠKA (2001). No primeiro deles, a solução do MEFG para um problema de Laplace em $\mathbb{R}^{2}$ tem o seu erro estimado mediante dois procedimentos: o MRE equilibrado e a diferença entre os gradientes da solução e de seu valor recuperado a partir de um ajuste realizado por mínimos quadrados. Discutese, apenas, a eficiência dos estimadores sem introduzi-los em um procedimento adaptativo. Em STROUBOULIS; COPPS; BABUŠKA (2001), por sua vez, um problema 
de Laplace em $\mathbb{R}^{2}$ é, novamente, analisado, considerando-se a presença de vazios no domínio. A abordagem dada aos estimadores de erro tem relação com a técnica de pósprocessamento dos gradientes mas, ao contrário do que é geralmente adotado naquela técnica, a função suavizada, ou recuperada, $\boldsymbol{u}_{p}^{\text {rec }}$, está associada, nesse caso, à própria solução $\boldsymbol{u}_{p}$ e não ao gradiente. Funções especiais podem ser incluídas e uma diferente solução suavizada, $\boldsymbol{u}^{\mathrm{rec}, \omega_{j}}$, é obtida para cada nuvem $\omega_{j}$, utilizando-se de ajuste por mínimos quadrados. Obtêm-se, então, diversas descrições de $\boldsymbol{u}^{\text {rec, } \omega_{j}}$ que se sobrepõem nas regiões de intercessão das nuvens. A "costura" entre estas descrições é realizada através das funções $\left\{\mathcal{N}_{j}\right\}_{j=1}^{N}$ definidoras da PU, da seguinte maneira:

$$
\boldsymbol{u}_{p}^{\mathrm{rec}}=\sum_{j=1}^{N} \mathcal{N}_{j} \boldsymbol{u}_{p}^{\mathrm{rec}, \omega_{j}}
$$

Chega-se, então, à uma função suavizada global $\boldsymbol{u}_{p}^{\text {rec }}$, que é empregada para o cálculo dos indicadores de erro. Tais indicadores e o estimador correspondente são usados dentro de um processo adaptativo do tipo $h$. A possibilidade de se utilizar um enriquecimento $p$-adaptativo não é explorada.

\subsubsection{Estimador de erro no MEFG - Escolha e Justificativa}

Desde o princípio deste trabalho, procurou-se priorizar a utilização de aproximações o máximo possível independentes de uma malha. Foi essa a razão da opção inicial pelo Método das Nuvens $h p$ e pelo MGLE, nos quais é suficiente a existência de pontos nodais distribuídos de forma adequada para a construção da aproximação. No MEFG, como já discutido no capítulo 2, a PU volta a ser definida sobre uma malha de elementos finitos. Por outro lado, pelos exemplos apresentados no capítulo 3, mostrou-se que a estratégia de enriquecimento reduz significativamente a importância da malha, especialmente no que se refere à convergência da aproximação. Dentro desse contexto, é natural que o algoritmo adaptativo implementado no MEFG procure preservar a configuração inicial da malha e utilize o enriquecimento polinomial para melhorar a aproximação. O estimador de erro deve, portanto, ser aplicável a esse tipo de abordagem seja qual for o grau polinomial da aproximação. Sendo assim, para a escolha do método de estimativa de erro foram consideradas as seguintes observações:

- Em BABUŠKA (1994a) são realizados extensivos testes numéricos com o método dos resíduos, nas versões explícita e implícita, bem como com os métodos de recuperação do gradiente da solução na versão inicial de ZIENKIEWICZ; 
ZHU (1987) e na versão super-convergente de ZIENKIEWICZ; ZHU (1992a), sendo esse último o que apresentou melhores resultados. Esta estratégia, entretanto, não se aplica com a mesma eficiência quando o grau do polinômio reproduzido pelo espaço das aproximações for de ordem mais elevada. Nos testes citados, por exemplo, foram utilizados somente elementos com aproximações linear e quadrática. Trabalhos como BOROOMAND; ZIENKIEWICZ (1997) e CARSTENSEN; FUNKEN (2000b) discutem interessantes alternativas a esses problemas, mas o assunto ainda se encontra em aberto;

- para a implementação de um procedimento adaptativo do tipo $p$ ou mesmo $h p$ o MRE é perfeitamente aplicável, o que pode ser averiguado, por exemplo em AINSWORTH; ODEN (1997) e ODEN (1989). Sua formulação, na qual a função de erro é projetada em espaços polinomiais mais enriquecidos, está diretamente ligada à possibilidade de se realizar refinamentos do tipo $p$;

- novamente em BABUŠKA (1994a), conclui-se que entre os estimadores de resíduo testados, o MRE na versão euilibrada é o mais robusto;

Diante das observações citadas, foi adotado, para a aplicação em análises adaptativas do tipo $p$, o MRE na versão equilibrada. A próxima seção é dedicada à descrição dessa estratégia de estimativa de erro, apresentada segundo a formulação bidimensional da elasticidade já empregada na seção 3.2.

\subsection{Método dos Resíduos em Elementos}

Por se tratar de uma aproximação, a solução numérica do problema variacional (3.13) não verifica, exatamente, as equações do PVC (3.11). Surge, então, a questão de se determinar, ou pelo menos estimar, o erro da solução obtida.

No MRE, funções resíduo, que medem a incapacidade da aproximação em verificar o PVC, são utilizadas para determinar os indicadores e o estimador de erro. A seguir, tal estratégia é apresentada de forma conceitual, com o objetivo de introduzir as expressões necessárias para sua implementação. O formalismo matemático, através do qual demonstra-se a convergência do estimador para o erro exato da solução, pode ser encontrado em ODEN (1989).

Considera-se, portanto, que o problema (3.13) tenha sido aproximado por funções pertencentes ao espaço $X_{p}$. Sejam, assim, a solução aproximada $\boldsymbol{u}_{p}$ e o erro de 
aproximação $e_{p}$. Representando-se a solução exata sob a seguinte forma:

$$
\boldsymbol{u}=\boldsymbol{e}_{p}+\boldsymbol{u}_{p}
$$

e utilizando-a em (3.11), tem-se que:

$$
\begin{aligned}
& \boldsymbol{\nabla}^{T} \boldsymbol{\Sigma}\left(\boldsymbol{e}_{p}+\boldsymbol{u}_{p}\right)+\boldsymbol{b}=\mathbf{0} \text { em } \Omega \\
& \boldsymbol{e}_{p}+\boldsymbol{u}_{p} \quad=\hat{\boldsymbol{u}} \quad \text { em } \Gamma_{D}
\end{aligned}
$$

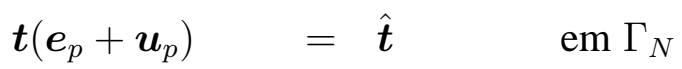

Ainda que não seja essencial para a formulação, assume-se que as condições de contorno de Dirichlet sejam satisfeitas de maneira exata pela aproximação $\boldsymbol{u}_{p}$ conforme AINSWORTH; ODEN (1997). Isto implica em se ter $\boldsymbol{u}_{p}=\hat{\boldsymbol{u}}$, ou seja, $\boldsymbol{e}_{p}=\mathbf{0}$ em $\Gamma_{D}$. Chega-se, então, a um novo PVC:

$$
\begin{aligned}
& \text { Encontrar } e_{p} \text { tal que: } \\
& \boldsymbol{\nabla}^{T} \boldsymbol{\Sigma}\left(\boldsymbol{e}_{p}\right)+\boldsymbol{r}_{\Omega}\left(\boldsymbol{u}_{p}\right)=\mathbf{0} \quad \mathrm{em} \Omega \\
& \begin{array}{llll}
\boldsymbol{e}_{p} & = & \mathbf{0} & \mathrm{em} \Gamma_{D}
\end{array} \\
& \boldsymbol{t}\left(\boldsymbol{e}_{p}\right) \quad=\boldsymbol{r}_{\Gamma}\left(\boldsymbol{u}_{p}\right) \quad \text { em } \Gamma_{N}
\end{aligned}
$$

onde são definidas os seguintes vetores resíduo no domínio e no contorno:

$$
\begin{array}{ll}
\boldsymbol{r}_{\Omega}\left(\boldsymbol{u}_{p}\right)=\nabla^{T} \boldsymbol{\Sigma}\left(\boldsymbol{u}_{p}\right)+\boldsymbol{b} & \text { em } \Omega \\
\boldsymbol{r}_{\Gamma}\left(\boldsymbol{u}_{p}\right)=\hat{\boldsymbol{t}}-\boldsymbol{t}\left(\boldsymbol{u}_{p}\right) & \mathrm{em} \Gamma_{N}
\end{array}
$$

Observa-se que $\boldsymbol{u}_{p} \in \mathcal{X}_{p} \subset \mathcal{H}^{1}$, não havendo garantias que $\boldsymbol{\nabla}^{T} \boldsymbol{\Sigma}\left(\boldsymbol{e}_{p}\right)$ exista em todas as regiões do domínio (na fronteira dos elementos por exemplo). Por essa razão, o problema (6.21) passa a ser interpretado a partir de sua forma fraca, isto é, o erro $\boldsymbol{e}_{p}$ tem significado apenas como solução da forma variacional associada. Para encontrá-la, parte-se, então, do seguinte problema:

$$
\begin{aligned}
& \text { Encontrar } \boldsymbol{e}_{p} \in \mathcal{H}^{1} \text { tal que: } \\
& \iint_{\Omega} \boldsymbol{v}^{T}\left\{\boldsymbol{\nabla}^{T} \boldsymbol{\Sigma}\left(\boldsymbol{e}_{p}\right)+\boldsymbol{r}_{\Omega}\left(\boldsymbol{u}_{p}\right)\right\} l_{z} d x d y=0 \quad \forall \quad \boldsymbol{v} \in \mathcal{H}^{1}
\end{aligned}
$$

onde $\boldsymbol{v}$ são funções teste de erro. Aplicando-se o teorema da divergência e escrevendo- 
se as integrais a partir das contribuições por elemento $\mathcal{K}$, tem-se que:

$$
\sum_{\mathcal{K} \in \Omega}\left\{\int_{\partial \mathcal{K}} \boldsymbol{v}^{T} \boldsymbol{t}\left(\boldsymbol{e}_{p}\right) l_{z} d s-\mathcal{B}_{\mathcal{K}}\left(\boldsymbol{e}_{p}, \boldsymbol{v}\right)+\iint_{\mathcal{K}} \boldsymbol{v}^{T} \boldsymbol{r}_{\Omega}\left(\boldsymbol{u}_{p}\right) l_{z} d x d y\right\}=0
$$

onde $\mathcal{B}_{\mathcal{K}}\left(\boldsymbol{e}_{p}, \boldsymbol{v}_{p}\right)=\iint_{\mathcal{K}} \varepsilon^{T}(\boldsymbol{v}) \boldsymbol{\sigma}\left(\boldsymbol{e}_{p}\right) l_{z} d x d y$, corresponde à parcela de contribuição no elemento $\mathcal{K}$ para $\mathcal{B}\left(\boldsymbol{e}_{p}, \boldsymbol{v}\right)$.

Empregando-se, então, a definição de $\boldsymbol{t}\left(\boldsymbol{e}_{p}\right)$ obtida de (6.21), chega-se a:

$$
\begin{gathered}
\sum_{\mathcal{K} \in \Omega} \mathcal{B}_{\mathcal{K}}\left(\boldsymbol{e}_{p}, \boldsymbol{v}\right)=\sum_{\mathcal{K} \in \Omega}\left\{\int_{\partial \mathcal{K} \cap \Gamma_{N}} \boldsymbol{v}^{T} \boldsymbol{r}_{\Gamma}\left(\boldsymbol{u}_{p}\right) l_{z} d s+\int_{\partial \mathcal{K} \backslash \partial \Omega} \boldsymbol{v}_{p}^{T} \boldsymbol{t}\left(\boldsymbol{e}_{p}\right) l_{z} d s\right. \\
\left.+\iint_{\mathcal{K}} \boldsymbol{v}^{T} \boldsymbol{r}_{\Omega}\left(\boldsymbol{u}_{p}\right) l_{z} d x d y\right\}
\end{gathered}
$$

onde $\partial \mathcal{K} \backslash \partial \Omega$ refere-se à parte do contorno $\partial \mathcal{K}$ do elemento $\mathcal{K}$ que não está contida no contorno $\partial \Omega$ do domínio $\Omega$.

A princípio, o problema acima poderia ser aproximado por Galerkin, para um espaço com dimensão superior à de $X_{p}$ em que a solução $\boldsymbol{u}_{p}$ é definida. O custo envolvido para a resolução de tal problema seria, contudo, equivalente ao de se repetir a análise para uma melhor discretização, o que inviabilizaria tal procedimento. Por essa razão, procura-se substituir (6.24) por problemas locais independentes, definidos para cada elemento $\mathcal{K}$. Surge, então, na fronteira entre elementos vizinhos uma nova condição de contorno natural a ser considerada:

$$
\boldsymbol{t}\left(\boldsymbol{e}_{p}\right)=\boldsymbol{t}(\boldsymbol{u})-\boldsymbol{t}\left(\boldsymbol{u}_{p}\right) \quad \text { em } \partial \mathcal{K} \backslash \partial \Omega
$$

Como não se conhece a função $\boldsymbol{t}(\boldsymbol{u})$, emprega-se como uma estimativa, a tensão média atuante sobre as faces do elemento $\mathcal{K}$. Dessa forma, em uma face qualquer de $\mathcal{K}$ pertencente, também, ao elemento vizinho $\mathcal{K}^{*}$, o valor médio das tensões é definido como:

$$
\left\langle\boldsymbol{t}\left(\boldsymbol{u}_{p}\right)\right\rangle_{m} \stackrel{\text { def }}{=} \frac{1}{2}\left\{\boldsymbol{t}\left(\boldsymbol{u}_{p}^{*}\right)+\boldsymbol{t}\left(\boldsymbol{u}_{p}\right)\right\}=\frac{1}{2}\left\{\boldsymbol{\sigma}\left(\boldsymbol{u}_{p}^{*}\right) \boldsymbol{n}^{\mathcal{K}}+\boldsymbol{\sigma}\left(\boldsymbol{u}_{p}\right) \boldsymbol{n}^{\mathcal{K}}\right\}
$$

onde o versor $\boldsymbol{n}^{\mathcal{K}}$ é normal às faces de $\mathcal{K}$, definido de modo a apontar para fora do elemento $\mathcal{K}$, e $\boldsymbol{u}_{p}^{*}$ é a aproximação para os deslocamentos no elemento $\mathcal{K}^{*}$. A condição 
de contorno (6.25) é, então, substituída por:

$$
\boldsymbol{t}\left(\boldsymbol{e}_{p}\right)=\left\langle\boldsymbol{t}\left(\boldsymbol{u}_{p}\right)\right\rangle_{m}-\boldsymbol{t}\left(\boldsymbol{u}_{p}\right)=\left[\boldsymbol{t}\left(u_{p}\right)\right] \quad \text { em } \partial \mathcal{K} \backslash \partial \Omega
$$

onde $\left[\boldsymbol{t}\left(u_{p}\right)\right]$ é à metade do salto das tensões na face entre os elementos $\mathcal{K}$ e $\mathcal{K}^{*}$ :

$$
\left[\boldsymbol{t}\left(u_{p}\right)\right] \stackrel{\text { def }}{=} \frac{1}{2}\left\{\boldsymbol{t}\left(\boldsymbol{u}_{p}^{*}\right)-\boldsymbol{t}\left(\boldsymbol{u}_{p}\right)\right\}
$$

Introduzindo-se a expressão (6.27) em (6.24), o seguinte problema de Neumman pode ser estabelecido para cada elemento $\mathcal{K}$ :

$$
\begin{aligned}
& \text { Encontrar } \boldsymbol{e}_{p} \in \mathcal{H}^{1}(\mathcal{K}) \text { tal que: } \\
& \qquad \begin{aligned}
\mathcal{B}_{\mathcal{K}}\left(\boldsymbol{e}_{p}, \boldsymbol{v}\right)=\int_{\partial \mathcal{K} \cap \Gamma_{N}} \boldsymbol{v}^{T} \boldsymbol{r}_{\Gamma}\left(\boldsymbol{u}_{p}\right) l_{z} d s+\int_{\partial \mathcal{K} \backslash \partial \Omega} \boldsymbol{v}^{T}\left[\boldsymbol{t}\left(\boldsymbol{u}_{p}\right)\right] l_{z} d s \\
\quad+\iint_{\mathcal{K}} \boldsymbol{v}^{T} \boldsymbol{r}_{\Omega}\left(\boldsymbol{u}_{p}\right) l_{z} d x d y
\end{aligned}
\end{aligned}
$$

Com base na condição (6.3), sabe-se que o erro é sempre ortogonal ao espaço das aproximações, $X_{p}$, no sentido do operador $\mathcal{B}(\bullet, \bullet)$. Por esta razão, o ideal seria procurar a solução para o problema (6.29) em um espaço ortogonal a $X_{p}$. Em face das dificuldades de fazê-lo, procura-se utilizar aproximações consistentes do próprio erro.

O espaço adotado em ODEN (1989) para se construir a aproximação de $\boldsymbol{e}_{p}$ é formado pelas funções bolhas do MEF hierárquico ${ }^{2}$, apresentando como características:

$$
X_{p+1}^{0}(\mathcal{K})=\left\{\boldsymbol{v}_{p+1}^{0} \in X_{p+1}(\mathcal{K}) ; \Pi_{p}\left(\boldsymbol{v}_{p+1}^{0}\right)=0 ; \boldsymbol{v}_{p+1}^{0}=0 \text { em } \partial \mathcal{K} \cap \Gamma_{D}\right\}
$$

onde $X_{p+1}(\mathcal{K})$ representa, de forma genérica, um espaço de funções definidas em $\mathcal{K}$ e com dimensão maior do que o espaço $X_{p}(\mathcal{K})$ onde a solução $\boldsymbol{u}_{p}$ é determinada. Na definição de $X_{p+1}^{0}(\mathcal{K})$ é utilizado o operador de interpolação local $\Pi_{p}$, que projeta funções do espaço $\mathcal{H}^{1}$ restritas ao elemento $\mathcal{K}$ em $\mathcal{X}_{p}(\mathcal{K})$, ou seja:

$$
\begin{aligned}
& \Pi_{p}: \mathcal{H}^{1}(\mathcal{K}) \rightarrow X_{p}(\mathcal{K}) \\
& \left.\boldsymbol{v}_{p}\right|_{\mathcal{K}}=\Pi_{p}(\boldsymbol{v})
\end{aligned}
$$

\footnotetext{
${ }^{2}$ Tais funções são denominadas em ODEN (1989) como bubble-like functions e abrangem as funções da formulação hierárquica do MEF, associadas aos nós de face e internos. Na verdade, apenas as funções associadas aos nós internos apresentam, realmente, a forma de uma bolha no elemento finito.
} 
Sendo assim, as funções $\boldsymbol{v}_{p+1}^{0}$ devem se anular em $\Gamma_{D}$ e pertencer ao espaço $\mathcal{X}_{p+1}(\mathcal{K})$, além de serem ortogonais a $X_{p}(\mathcal{K})$ no sentido definido por $\Pi_{p}$. No apêndice $\mathrm{G}$ a escolha desse espaço de funções recebe uma maior atenção. Neste momento, porém, basta entender que a função de erro $e_{p}$ está sendo aproximada por um conjunto de funções $\tilde{\boldsymbol{e}}_{p} \in X_{p+1}^{0}(\mathcal{K})$ definidas para cada elemento $\mathcal{K}$ e obtidas do seguinte problema:

Encontrar $\tilde{\boldsymbol{e}}_{p} \in X_{p+1}^{0}(\mathcal{K})$ tal que:

$$
\begin{aligned}
& \mathcal{B}_{\mathcal{K}}\left(\tilde{\boldsymbol{e}}_{p}, \boldsymbol{v}_{p+1}^{0}\right)=\int_{\partial \mathcal{K} \cap \Gamma_{N}}\left(\boldsymbol{v}_{p+1}^{0}\right)^{T} \boldsymbol{r}_{\Gamma}\left(\boldsymbol{u}_{p}\right) l_{z} d s \\
& \quad+\int_{\partial \mathcal{K} \backslash \partial \Omega}\left(\boldsymbol{v}_{p+1}^{0}\right)^{T}\left[\boldsymbol{t}\left(\boldsymbol{u}_{p}\right)\right] l_{z} d s+\iint_{\mathcal{K}}\left(\boldsymbol{v}_{p+1}^{0}\right)^{T} \boldsymbol{r}_{\Omega}\left(\boldsymbol{u}_{p}\right) l_{z} d x d y
\end{aligned}
$$

A presença de $\boldsymbol{r}_{\Omega}$ em (6.32) implica no envolvimento de derivadas de segunda ordem da função $\boldsymbol{u}_{p}$. Para evitar a perda de informação, especialmente quando $\boldsymbol{u}_{p}$ for linear, propõe-se, em DUARTE (1991), uma simplificação de (6.32), integrando-se por partes a parcela do resíduo. Chega-se, assim, à descrição final da aproximação de Galerkin do problema (6.29):

$$
\begin{aligned}
& \text { Encontrar } \boldsymbol{e}_{p} \in X_{p+1}^{0}(\mathcal{K}) \text { tal que: } \\
& \qquad \mathcal{B}_{\mathcal{K}}\left(\boldsymbol{e}_{p}, \boldsymbol{v}_{p+1}^{0}\right)=\mathcal{L}_{\mathcal{K}}\left(\boldsymbol{v}_{p+1}^{0}\right) \forall \boldsymbol{v}_{p+1}^{0} \in X_{p+1}^{0}(\mathcal{K})
\end{aligned}
$$

onde

$$
\begin{aligned}
\mathcal{L}_{\mathcal{K}}\left(\boldsymbol{v}_{p+1}^{0}\right) \stackrel{\text { def }}{=} \iint_{\mathcal{K}}\left(\boldsymbol{v}_{p+1}^{0}\right)^{T} \boldsymbol{b} l_{z} d x d y-\mathcal{B}_{\mathcal{K}}\left(\boldsymbol{u}_{p}, \boldsymbol{v}_{p+1}^{0}\right) \\
+\int_{\partial \mathcal{K} \cap \Gamma_{N}}\left(\boldsymbol{v}_{p+1}^{0}\right)^{T} \hat{\boldsymbol{t}} l_{z} d s+\int_{\partial \mathcal{K} \backslash \partial \Omega}\left(\boldsymbol{v}_{p+1}^{0}\right)^{T}\left\langle\boldsymbol{t}\left(\boldsymbol{u}_{p}\right)\right\rangle_{m} l_{z} d s
\end{aligned}
$$

Até este ponto, as formulações do MRE foram apresentadas para o MEF hierárquico. Um problema equivalente pode ser definido se o espaço de funções bolha (6.30) for construído a partir das funções de forma do MEFG. Neste texto, o espaço gerado por tais funções é também referido como espaço de funções bolha e representado, de forma análoga, por $X_{p+1}^{0}(\mathcal{K})$. Deve-se observar, entretanto, que as funções de enriquecimento do MEFG não apresentam exatamente a forma de uma bolha. Anulam-se, contudo, nos vértices assim como as funções do MEF hierárquico. Além disso, a repre- 
sentação adotada restringe o espaço ao elemento $\mathcal{K}$, quando na realidade as funções de enriquecimento são associadas às nuvens, sendo construídas globalmente. O problema (6.33), entretanto, continua a ser resolvido para o elemento e, por isso, o espaço de funções utilizado no MEFG mantém a mesma representação do MEF, ou seja, $X_{p+1}^{0}(\mathcal{K})$, em alusão ao elemento em que se calcula a função erro aproximada.

As funções $\tilde{\boldsymbol{e}}_{p}$ e $\boldsymbol{v}_{p+1}^{0}$ podem, portanto, ser obtidas pelas funções do MEFG como:

$$
\begin{array}{r}
\tilde{\boldsymbol{e}}_{p}=\left(\boldsymbol{\Phi}_{p+1}^{0}\right)^{T} \boldsymbol{I} \\
\boldsymbol{v}_{p+1}^{0}=\left(\boldsymbol{\Phi}_{p+1}^{0}\right)^{T} \boldsymbol{V}^{0}
\end{array}
$$

onde os seguintes vetores e matrizes são definidos, sendo o primeiro deles denominado vetor dos indicadores de erro nodais:

$$
\begin{aligned}
& \left(\boldsymbol{I}^{\mathcal{K}}\right)^{T} \stackrel{\text { def }}{=}\left[\begin{array}{lllllll}
b_{1 q_{1}(p)+1}^{x} & b_{1 q_{1}(p)+1}^{y} & \cdots & b_{1 q_{1}(p+1)}^{x} & b_{1 q_{1}(p+1)}^{y} & \cdots
\end{array}\right. \\
& \left.b_{N q_{N}(p)+1}^{x} \quad b_{N q_{N}(p)+1}^{y} \quad \cdots \quad b_{N q_{N}(p+1)}^{x} \quad b_{N q_{N}(p+1)}^{y}\right] \\
& \left(\boldsymbol{V}^{0}\right)^{T} \stackrel{\text { def }}{=}\left[\begin{array}{lllllll}
c_{1 q_{1}(p)+1}^{x} & c_{1 q_{1}(p)+1}^{y} & \cdots & c_{1 q_{1}(p+1)}^{x} & c_{1 q_{1}(p+1)}^{y} & \cdots
\end{array}\right. \\
& \left.\begin{array}{ccccc}
c_{N q_{N}(p)+1}^{x} & c_{N q_{N}(p)+1}^{y} & \cdots & c_{N q_{N}(p+1)}^{x} & c_{N q_{N}(p+1)}^{y}
\end{array}\right] \\
& \left(\boldsymbol{\Phi}_{p+1}^{0}\right)^{T} \stackrel{\text { def }}{=}\left[\begin{array}{cccc}
p_{1 q_{1}(p)+1} \mathcal{N}_{1} & 0 & \cdots & p_{1 q_{1}(p+1)} \mathcal{N}_{q_{1}(p+1)} \\
0 & p_{1 q_{1}(p)+1} \mathcal{N}_{1} & \cdots & 0
\end{array}\right. \\
& \begin{array}{llll}
0 & \cdots & p_{N q_{N}(p)+1} \mathcal{N}_{N} \quad 0
\end{array} \\
& \begin{array}{rrrr}
p_{1 q_{1}(p+1)} \mathcal{N}_{q_{1}(p+1)} & \cdots & 0 & p_{N q_{N}(p)+1} \mathcal{N}_{N}
\end{array} \\
& \left.\begin{array}{ccc}
\cdots & p_{N q_{N}(p+1)} \mathcal{N}_{p_{N}(p+1)} & 0 \\
\cdots & 0 & p_{N q_{N}(p+1)} \mathcal{N}_{p_{N}(p+1)}
\end{array}\right]
\end{aligned}
$$

Observa-se que $\left(\boldsymbol{\Phi}_{p+1}^{0}\right)$ é obtido a partir da definição (3.18), eliminando-se das funções de forma $\left(\boldsymbol{\Phi}_{p+1}\right)$ os termos presentes em $\left(\boldsymbol{\Phi}_{p}\right)^{T}$, que representam polinômios completos de grau $p+1$ e $p$ respectivamente. 
O seguinte sistema de equações deve então ser resolvido para cada elemento $\mathcal{K}$ :

$$
\boldsymbol{K}_{\mathrm{er}}^{\mathcal{K}} \boldsymbol{I}^{\mathcal{K}}=\boldsymbol{R}^{\mathcal{K}}
$$

onde são definidos de forma análoga à seção (3.2):

- matriz de rigidez:

$$
\boldsymbol{K}_{\mathrm{er}}^{\mathcal{K}}=\iint_{\mathcal{K}}\left(\boldsymbol{B}_{p+1}^{0}\right)^{T} \boldsymbol{C} \boldsymbol{B}_{p+1}^{0} l_{z} d x d y
$$

- vetor de forças residuais nodais generalizadas:

$$
\begin{aligned}
\boldsymbol{R}^{\mathcal{K}} & =\iint_{\mathcal{K}}\left(\boldsymbol{\Phi}_{p+1}^{0}\right) \boldsymbol{b} l_{z} d x d y-\boldsymbol{K}^{\mathcal{K}} \boldsymbol{U}+\int_{\partial \mathcal{K} \cap \Gamma_{N}}\left(\boldsymbol{\Phi}_{p+1}^{0}\right) \hat{\boldsymbol{t}} l_{z} d s \\
& +\int_{\partial \mathcal{K} \backslash \partial \Omega}\left(\boldsymbol{\Phi}_{p+1}^{0}\right)\left\langle\boldsymbol{t}\left(\boldsymbol{u}_{p}\right)\right\rangle_{m} l_{z} d s
\end{aligned}
$$

- matriz de rigidez do elemento:

$$
\boldsymbol{K}^{\mathcal{K}}=\iint_{\mathcal{K}}(\boldsymbol{B})^{T} \boldsymbol{C B} l_{z} d x d y
$$

- operador:

$$
\boldsymbol{B}_{p+1}^{0}=\boldsymbol{L}\left(\boldsymbol{\Phi}_{p+1}^{0}\right)^{T}
$$

Conhecida então a função $\tilde{e}_{p}$, para cada elemento $\mathcal{K}$, pode-se determinar de (6.5), a norma energia local:

$$
\tilde{\mathcal{E}}_{\mathcal{K}} \stackrel{\text { def }}{=}\left\|\tilde{\boldsymbol{e}}_{p}\right\|_{u(\mathcal{K})}=\left[\mathcal{B}_{\mathcal{K}}\left(\tilde{\boldsymbol{e}}_{p}, \tilde{\boldsymbol{e}}_{p}\right)\right]^{1 / 2}
$$

em que $\tilde{\mathcal{E}}_{\mathcal{K}}$ são os indicadores de erro, (6.10) e, por analogia, as funções $\tilde{\boldsymbol{e}}_{p}$ são denominadas de funções indicadoras de erro. O estimador de erro global é obtido através da expressão (6.6) pela contribuição dos indicadores locais, como já mostrado em (6.11) e repetido a seguir: 


$$
\left\|\tilde{\boldsymbol{e}}_{p}\right\|_{u}=\sqrt{\sum_{\mathcal{K} \in \Omega} \tilde{\mathcal{E}}_{\mathcal{K}}^{2}}
$$

$\mathrm{Na}$ abordagem do MEFG, torna-se interessante que se defina uma medida de erro associada a cada nuvem $\omega_{j}$, uma vez que o enriquecimento está vinculado aos nós e não mais aos elementos. Como o sistema (6.40) é resolvido para cada elemento $\mathcal{K}$, a parcela $\boldsymbol{I}_{\omega_{j}}$ de $\boldsymbol{I}_{\mathcal{K}}$ associada à nuvem $\omega_{j}$ somente é válida no respectivo elemento $\mathcal{K}$. Para os elementos vizinhos de $\mathcal{K}$ e que também pertençam à nuvem $\omega_{j}$ haverá outros valores para $\boldsymbol{I}_{\omega_{j}}$. Não existe, portanto, coerência em se calcular a norma energia de uma função que estaria associada a cada nuvem $\omega_{j}$ e definida de maneira múltipla para cada elemento dessa nuvem ${ }^{3}$. Para se ter tal medida, optou-se, então, por calcular a média ponderada dos indicadores de erro, (6.45), de todos os elementos $\mathcal{K}$ contidos na nuvem $\omega_{j}$, usando, como peso, o volume $V_{\mathcal{K}}$ desses elementos. Dessa forma, define-se o indicador de erro por nuvem como:

$$
\tilde{\mathcal{E}}_{\omega_{j}} \stackrel{\text { def }}{=} \sum_{\mathcal{K} \in \omega_{j}} \frac{V_{\mathcal{K}}\left\|\tilde{\boldsymbol{e}}_{p}\right\|_{\mathcal{u}(\mathcal{K})}}{V_{\omega_{j}}}
$$

onde $V_{\omega_{j}}=\sum_{\mathcal{K} \in \omega_{j}} V_{\mathcal{K}}$ corresponde ao volume total da nuvem $\omega_{j}$.

\subsection{Equilíbrio dos Resíduos}

O problema (6.32), que origina (6.33), corresponde para elementos no interior de $\Omega$ a um problema de Neumman pois apresenta apenas as condições de contorno naturais. Por essa razão, dependendo de como as expressões do lado direito são construídas, uma solução única pode não ser definida. O emprego do espaço $X_{p+1}^{0}$ resolve, em parte, esse problema, eliminado-se componentes da função $\tilde{\boldsymbol{e}}_{p}$ que corresponderiam a modos equivalentes aos movimentos de corpo rígido. A formulação, entretanto, permanece deficitária e, segundo AINSWORTH; ODEN (1997), é necessário que o problema seja bem posto. Para isso, os dados, $\left(\boldsymbol{r}_{\Omega}, \boldsymbol{r}_{\Gamma},\left[\boldsymbol{t}\left(\boldsymbol{u}_{p}\right)\right]\right)$, utilizados para o PVC do erro devem ser alterados de tal maneira que se tornem auto-equilibrados.

Várias são as propostas para se impor o equilíbrio entre os dados calculados no contorno e aqueles definidos no domínio de cada elemento. Entre elas podem ser citadas as de OHTSUBO; KITAMURA (1992), OHTSUBO; KITAMURA (1990) e LA-

\footnotetext{
${ }^{3}$ Uma alternativa que talvez merecesse atenção consiste em se definir o problema (6.23) não mais para o elemento mas para a nuvem, o que conduziria diretamente ao indicador de erro por nuvens.
} 
DEVĖZE; MAUNDER (1996). Nos dois primeiros trabalhos, o equilíbrio é realizado isoladamente em cada elemento, e é descrito, na formulação do MEF, para aproximações Lagrangianas e Serendípetas. Uma técnica de otimização é aplicada para corrigir o vetor das forças residuais (6.42) que passa a ter componentes nulas de força e momento no elemento finito correspondente. Sua implementação é bastante simples e implica em pequeno custo computacional. Não é, contudo, aplicável à versão hierárquica do MEF pois nem todas as componentes do vetor equilibrado correspondem fisicamente a forças. Também no caso de LADEVÈZE; MAUNDER (1996), a estratégia sugerida é aplicada sobre o vetor de forças residuais, mas procurando-se, indiretamente, equilibrar os dados do problema. Em uma formulação hierárquica, é possível equilibrar apenas as componentes de $\boldsymbol{R}_{\mathcal{K}}$ que mantêm a correspondência física com as forças. O processo de equilíbrio em cada elemento envolve, entretanto, todos os seus elementos vizinhos o que torna sua implementação mais onerosa do ponto de vista computacional.

Como a construção das funções de aproximação no MEFG também se dá de forma hierárquica, optou-se pela implementação da estratégia de Ladevèze \& Maunder, sendo esse o assunto da próxima seção.

\subsubsection{Estratégia de Equilíbrio de Ladevèze \& Maunder}

Em LADEVÈZE; MAUNDER (1996) utiliza-se do conceito de forças nodais e do diagrama de forças de Maxwell para apresentar, geometricamente, uma estratégia que recupera, no elemento finito, um campo de tensões equilibradas. Aplicada aos resíduos de forças no contorno e no domínio, tal estratégia torna possível alterar o vetor $\boldsymbol{R}^{\mathcal{K}}$ para o problema (6.40) tornando-o bem posto. Não é objetivo deste trabalho esmiuçar esse assunto e, por isso, apenas uma idéia geral será apresentada.

Sabe-se da seção 6.3, que o vetor $\boldsymbol{R}^{\mathcal{K}},(6.42)$, é construído a partir da expressão (6.32) em que participam os dados de resíduo $\boldsymbol{r}_{\Omega}, \boldsymbol{r}_{\Gamma}$, calculados no elemento $\mathcal{K}$, e do salto de tensões nas faces de $\mathcal{K}$, representado por $\left[\boldsymbol{t}\left(\boldsymbol{u}_{p}\right)\right]$. Geralmente, esse último dado não se encontra em equilíbrio com os primeiros e, por isso, torna-se necessário que tensões de correção, $\boldsymbol{\theta}^{\mathcal{K}}$, sejam distribuídas ao longo dos lados do elemento $\mathcal{K}$, de modo que se tenha resultante nula para as forças: 


$$
\iint_{\mathcal{K}} \boldsymbol{r}_{\Omega} l_{z} d x d y+\int_{\partial \mathcal{K} \cap \Gamma_{N}} \boldsymbol{r}_{\Gamma} l_{z} d s+\int_{\partial \mathcal{K} \backslash \partial \Omega}\left[\boldsymbol{t}\left(\boldsymbol{u}_{p}\right)\right] l_{z} d s+\int_{\partial \mathcal{K}} \boldsymbol{\theta}^{\mathcal{K}} l_{z} d s=\mathbf{0}
$$

e para o momento:

$$
\begin{gathered}
\iint_{\mathcal{K}}\left(\rho^{y} r_{\Omega}^{x}+\rho^{x} r_{\Omega}^{y}\right) l_{z} d x d y+\int_{\partial \mathcal{K} \cap \partial \Omega}\left(\rho^{y} r_{\Gamma}^{x}+\rho^{x} r_{\Gamma}^{y}\right) l_{z} d s \\
+\int_{\partial \mathcal{K} \backslash \partial \Omega}\left(\rho^{y}\left[t^{x}\left(\boldsymbol{u}_{p}\right)\right]+\rho^{x}\left[t^{y}\left(\boldsymbol{u}_{p}\right)\right]\right) l_{z} d s+\int_{\partial \mathcal{K}}\left(\rho^{y} \theta_{x}^{\mathcal{K}}+\rho^{x} \theta_{y}^{\mathcal{K}}\right) l_{z} d s=\mathbf{0}
\end{gathered}
$$

onde é assumido o sentido horário como positivo para o momento e $\rho^{x}$ e $\rho^{y}$ são as distâncias em $x$ e em $y$ entre um ponto $\boldsymbol{x}$ de $\mathcal{K}$ e uma posição qualquer $\boldsymbol{x}_{0}$ do domínio $\Omega$.

Encontrar as distribuições de tensões $\boldsymbol{\theta}^{\mathcal{K}}$ que corrijam o equilíbrio em cada elemento $\mathcal{K}$ e que não violem o balanço de forças dos nós não é tarefa simples de ser realizada. Procurando realizá-la e também buscando reduzir o problema à solução de um conjunto finito de equações, Ladevèze \& Maunder utilizam-se, fundamentalmente, do conceito de forças nodais equivalentes.

Dessa forma, para uma aproximação $\boldsymbol{u}_{p}$, o vetor de forças equivalentes de um nó $\boldsymbol{x}_{j}$ no elemento $\mathcal{K}$ pode ser definido como:

$$
\boldsymbol{F}_{j}^{\mathcal{K}} \stackrel{\text { def }}{=} \iint_{\mathcal{K}} \boldsymbol{\phi}_{j} \boldsymbol{r}_{\Omega} l_{z} d x d y+\int_{\partial \mathcal{K} \cap \Gamma_{N}} \boldsymbol{\phi}_{j} \boldsymbol{r}_{\Gamma} l_{z} d s+\int_{\partial \mathcal{K} \backslash \partial \Omega} \boldsymbol{\phi}_{j}\left[\boldsymbol{t}\left(u_{p}\right)\right] l_{z} d s
$$

onde $\phi_{j}^{T}$ é uma sub-matriz de (3.21) que contém as funções $\phi_{j}$ associadas ao nó $\boldsymbol{x}_{j}$ nas direções $x$ e $y$ :

$$
\phi_{j}^{T}=\left[\begin{array}{ccccc}
\mathcal{N}_{j} & 0 & L_{j 1} \mathcal{N}_{j} \cdots & L_{j q_{j}} \mathcal{N}_{j} & 0 \\
0 & \mathcal{N}_{j} & 0 & \cdots & L_{j q_{j}} \mathcal{N}_{j}
\end{array}\right]
$$

As componentes de $\boldsymbol{F}_{j}^{\mathcal{K}}$ representam, então, os dados $\left(\boldsymbol{r}_{\Omega}, \boldsymbol{r}_{\Gamma},\left[\boldsymbol{t}\left(\boldsymbol{u}_{p}\right)\right]\right)$ através de valores nodais, podendo-se mostrar que:

$$
\sum_{\mathcal{K} \in \omega_{j}} \boldsymbol{F}_{j}^{\mathcal{K}}=\mathbf{0}
$$


ou seja, as forças nodais que incidem sobre o nó $\boldsymbol{x}_{j}$, computadas a partir dos elementos que o contêm, estão sempre em equilíbrio.

No apêndice $\mathrm{H}$, demonstra-se que se os vetores $\boldsymbol{F}_{j}^{\mathcal{K}}$ tiverem resultantes nulas em força e momento, o equilíbrio do conjunto de dados $\left(\boldsymbol{r}_{\Omega}, \boldsymbol{r}_{\Gamma},\left[\boldsymbol{t}\left(\boldsymbol{u}_{p}\right)\right]\right)$ fica também assegurado. Com base nessa observação, as tensões $\boldsymbol{\theta}^{\mathcal{K}}$ podem ser definidas como aquelas que verificam a seguinte expressão, para cada nó do elemento $\mathcal{K}$ :

$$
\boldsymbol{\Theta}_{j}^{\mathcal{K}}+\iint_{\mathcal{K}} \boldsymbol{\phi}_{j} \boldsymbol{r}_{\Omega} l_{z} d x d y+\int_{\partial \mathcal{K} \cap \Gamma_{N}} \boldsymbol{\phi}_{j} \boldsymbol{r}_{\Gamma} l_{z} d s+\int_{\partial \mathcal{K} \backslash \partial \Omega} \boldsymbol{\phi}_{j}\left[\boldsymbol{t}\left(u_{p}\right)\right] l_{z} d s=\mathbf{0}
$$

sendo:

$$
\Theta_{j}^{\mathcal{K}} \stackrel{\text { def }}{=} \int_{\partial \mathcal{K}} \phi_{j} \boldsymbol{\theta}^{\mathcal{K}} l_{z} d s
$$

Observa-se que o equilíbrio é total, inclusive para o momento, pois todas as componentes de (6.53) para todos os nós de $\mathcal{K}$ são anuladas. Caso isso se verifique, o novo conjunto de dados $\left(\boldsymbol{r}_{\Omega}, \boldsymbol{r}_{\Gamma},\left[\boldsymbol{t}\left(\boldsymbol{u}_{p}\right)\right], \boldsymbol{\theta}^{\mathcal{K}}\right)$ fica, portanto, indiretamente equilibrado. No apêndice $H$, mostra-se, também, que as componentes de $\Theta_{j}^{\mathcal{K}}$ vinculadas às funções de aproximação enriquecidas pelo MEFG não alteram as condições de equilíbrio, mas apenas aquelas associadas diretamente com a PU. Não é necessário, portanto, quando houver o enriquecimento, que a distribuição $\boldsymbol{\theta}^{\mathcal{K}}$ anule todas as componentes em (6.50). Por essa razão, um novo vetor $\hat{\Theta}_{j}^{\mathcal{K}}$, denominado vetor das forças nodais de correção do equilíbrio, é definido:

$$
\hat{\boldsymbol{\Theta}}_{j}^{\mathcal{K}} \stackrel{\text { def }}{=} \int_{\partial \mathcal{K}} \boldsymbol{N}_{j} \boldsymbol{\theta}^{\mathcal{K}} l_{z} d s
$$

onde é empregada a sub-matriz com as funções de interpolação do nó $\boldsymbol{x}_{j}$ do elemento $\mathcal{K}$ :

$$
\boldsymbol{N}=\left[\begin{array}{cc}
\mathcal{N}_{j} & 0 \\
0 & \mathcal{N}_{j}
\end{array}\right]
$$

A expressão (6.53) pode ser, então, reescrita como: 


$$
\hat{\boldsymbol{\Theta}}_{j}^{\mathcal{K}}+\iint_{\mathcal{K}} \boldsymbol{N}_{j} \boldsymbol{r}_{\Omega} l_{z} d x d y+\int_{\partial \mathcal{K} \cap \Gamma_{N}} \boldsymbol{N}_{j} \boldsymbol{r}_{\Gamma} l_{z} d s+\int_{\partial \mathcal{K} \backslash \partial \Omega} \boldsymbol{N}_{j}\left[\boldsymbol{t}\left(u_{p}\right)\right] l_{z} d s=\mathbf{0}
$$

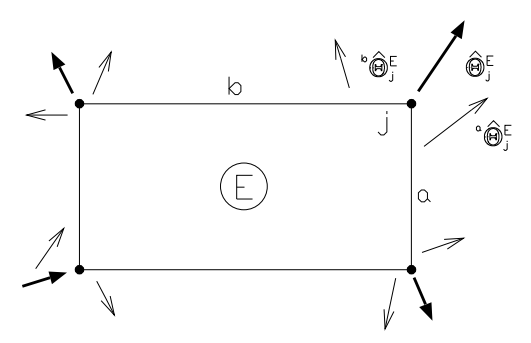

Figura 6.1: Decomposição de $\hat{\boldsymbol{\Theta}}_{j}^{\mathcal{K}}={ }^{a} \hat{\boldsymbol{\Theta}}_{j}^{\mathcal{K}}+{ }^{b} \hat{\boldsymbol{\Theta}}_{j}^{\mathcal{K}}$

Uma segunda etapa do procedimento, por assim dizer, é agora introduzida e consiste em se obter, em cada face compartilhada por dois elementos vizinhos, uma mesma distribuição de tensões de correção. Para isso, considera-se o elemento quadrilátero, $\mathcal{K}=E$, da Figura 6.1. Em cada vértice $j, \hat{\Theta}_{j}^{E}$ pode ser divido em duas parcelas ${ }^{a} \hat{\boldsymbol{\Theta}}_{j}^{E}$ $\mathrm{e}^{b} \hat{\boldsymbol{\Theta}}_{j}^{E}$, associadas a cada um dos dois lados $a$ e $b$ que contêm o nó $\boldsymbol{x}_{j}$. Para se garantir a definição única das tensões de correção, é necessário que tais parcelas sejam definidas com o mesmo módulo mas de sentido contrário às correspondentes nos elementos vizinhos. Este processo de balanceamento não pode se restringir a um único elemento, devendo ser considerada a interação entre todos os elementos da nuvem que contém o nó $\boldsymbol{x}_{j}$, como ilustra a Figura 6.2(a).

Para melhor entender esse processo, recorre-se a uma representação geométrica através do diagrama de Maxwell. Na Figura 6.2(b), por exemplo, encontram-se presentes as forças nodais de correção e sua decomposição em parcelas para cada lado dos elementos que compartilham o nó $\boldsymbol{x}_{j}$. Seja qual for a posição $\boldsymbol{x}_{p}$, as parcelas de força assim obtidas garantem a continuidade das tensões de correção entre elementos. Uma alternativa é se calcular o ponto $\boldsymbol{x}_{p}$ como o centróide das massas $\left(m_{a}, m_{b}, m_{c}, m_{d}\right)$ que são, na realidade, pesos escolhidos conforme a estratégia adotada. Para as análises numéricas apresentadas nas seções (6.5) e (6.7) adota-se, conforme utilizado em LADEVÈZE; MAUNDER (1996):

$$
m_{\alpha}=\frac{1}{L_{\alpha}^{2}}
$$



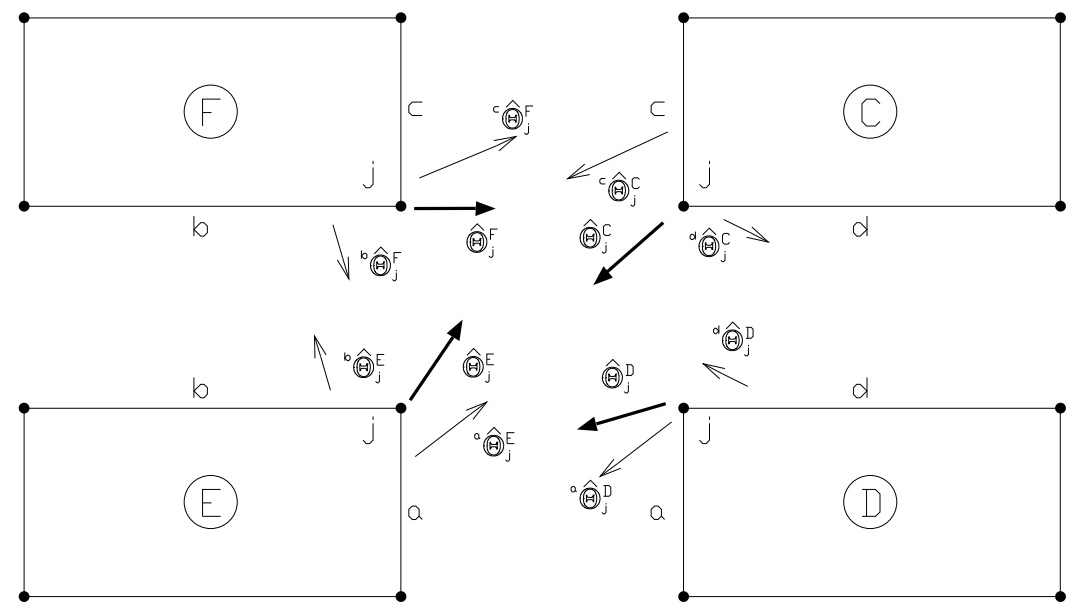

(a) Nuvem $\omega_{j}$ e a decomposição das forças residuais nodais

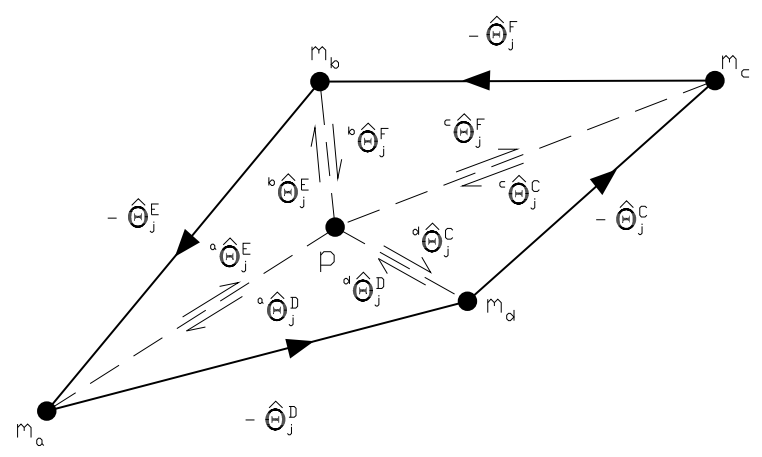

(b) Equilíbrio nodal - Diagrama de Maxwell

Figura 6.2: Processo de balanceamento das forças $\hat{\Theta}_{j}^{\mathcal{K}}$ respeitando-se o equilíbrio nodal 
onde $L_{\alpha}$ corresponde ao tamanho do lado $\alpha=a, b, c, d$

Conhecendo-se, então, as parcelas de forças nodais ${ }^{\alpha} \Theta_{j}^{\mathcal{K}}$, para as faces $\alpha=$ $a, b, c, d$ que compartilham o nó $\boldsymbol{x}_{j}$ retorna-se à expressão (6.55), obtendo-se, para cada elemento $\mathcal{K}=C, D, E, F$ :

$$
{ }^{\alpha} \hat{\boldsymbol{\Theta}}_{j}^{\mathcal{K}}=\int_{\alpha} \boldsymbol{N}_{j}{ }^{\alpha} \boldsymbol{\theta}^{\mathcal{K}} l_{z} d s
$$

onde ${ }^{\alpha} \boldsymbol{\theta}_{\mathcal{K}}$ corresponde à distribuição de tensão $\boldsymbol{\theta}$ presente na face $\alpha$ do elemento $\mathcal{K}$. Assume-se que cada uma dessas tensões sejam geradas pelas funções da PU, ou seja:

$$
{ }^{\alpha} \boldsymbol{\theta}^{\mathcal{K}}=\mathcal{N}_{j}^{\mathcal{K} \alpha} \boldsymbol{\theta}_{j}^{\mathcal{K}}+\mathcal{N}_{i}^{\mathcal{K} \alpha} \boldsymbol{\theta}_{i}^{\mathcal{K}}
$$

sendo $i$ e $j$ os vértices da face $\alpha$.

Chega-se, então, para cada face $\alpha$, ao seguinte sistema de equações:

$$
\boldsymbol{A}_{\mathcal{N}} \boldsymbol{b}_{\theta}=\boldsymbol{c}_{\Theta}
$$

sendo:

$$
\boldsymbol{A}_{\mathcal{N}}=\left[\begin{array}{ccc}
\int_{\alpha}\left(\mathcal{N}_{j}^{\mathcal{K}}\right)^{2} l_{z} d s \quad \int_{\alpha}\left(\mathcal{N}_{j}^{\mathcal{K}} \mathcal{N}_{i}^{\mathcal{K}}\right) l_{z} d s & 0 \\
\int_{\alpha}\left(\mathcal{N}_{i}^{\mathcal{K}} \mathcal{N}_{j}^{\mathcal{K}}\right) l_{z} d s & \int_{\alpha}\left(\mathcal{N}_{j}^{\mathcal{K}}\right)^{2} l_{z} d s & 0 \\
0 & \int_{\alpha}\left(\mathcal{N}_{j}^{\mathcal{K}}\right)^{2} l_{z} d s & \int_{\alpha}\left(\mathcal{N}_{j}^{\mathcal{K}} \mathcal{N}_{i}^{\mathcal{K}}\right) l_{z} d s \\
0 & \int_{\alpha}\left(\mathcal{N}_{j}^{\mathcal{K}} \mathcal{N}_{i}^{\mathcal{K}}\right) l_{z} d s \int_{\alpha}\left(\mathcal{N}_{j}^{\mathcal{K}}\right)^{2} l_{z} d s
\end{array}\right]
$$


onde $(\bullet)_{x} \mathrm{e}(\bullet)_{y}$ correspondem às componentes nas direções $x$ e $y \mathrm{e}^{\alpha} \hat{\Theta}_{j}^{\mathcal{K}} \mathrm{e}^{\alpha} \hat{\theta}_{j}^{\mathcal{X}}$ são elementos dos vetores ${ }^{\alpha} \boldsymbol{\Theta}^{\mathcal{K}} \mathrm{e}^{\alpha} \boldsymbol{\theta}^{\mathcal{K}}$ respectivamente.

Conhecidos os valores de ${ }^{\alpha} \boldsymbol{\theta}_{j}^{\mathcal{K}}$ para cada face $\alpha$ e nó $\boldsymbol{x}_{j}$ do elemento $\mathcal{K}$, as tensões de correção $\theta^{\mathcal{K}}$ são construídas através da expressão (6.60). O novo conjunto de dados $\left(\boldsymbol{r}_{\Omega}, \boldsymbol{r}_{\Gamma},\left[\boldsymbol{t}\left(u_{p}\right)\right], \boldsymbol{\theta}^{\mathcal{K}}\right)$ pode ser, então, empregado para o problema (6.32), gerando o seguinte sistema de equações, agora bem posto:

$$
\boldsymbol{K}^{\mathcal{K}} \boldsymbol{I}^{\mathcal{K}}=\boldsymbol{R}_{\text {equi }}^{\mathcal{K}}
$$

em que:

$$
\begin{aligned}
\boldsymbol{R}_{\text {equi }}^{\mathcal{K}} & =\iint_{\mathcal{K}}\left(\Phi_{p+1}^{0}\right) \boldsymbol{b} l_{z} d x d y-\boldsymbol{K}^{\mathcal{K}} \boldsymbol{U}+\int_{\partial \mathcal{K} \cap \Gamma_{N}}\left(\boldsymbol{\Phi}_{p+1}^{0}\right)^{T} \hat{\boldsymbol{t}} l_{z} d s \\
& +\int_{\partial \mathcal{K} \backslash \partial \Omega}\left(\boldsymbol{\Phi}_{p+1}^{0}\right)\left\langle\boldsymbol{t}\left(\boldsymbol{u}_{p}\right)\right\rangle_{m} l_{z} d s+\int_{\partial \mathcal{K}}\left(\boldsymbol{\Phi}_{p+1}^{0}\right) \boldsymbol{\theta}^{\mathcal{K}} l_{z} d s
\end{aligned}
$$

\subsubsection{Algoritmo Adaptativo}

Os algoritmos adaptativos utilizados, 6.1 e 6.2, correspondem à versão $p$, utilizado em DUARTE (1991) e ODEN (1989), e fundamentam-se no princípio da eqüidistribuição da norma do erro pelo domínio aproximado. O erro é controlado em dois níveis, um global através do estimador (6.46) e outro local por meio de indicadores de erro. O procedimento adaptativo prossegue enquanto o erro relativo estimado global $\tilde{\mathcal{E}}_{\%},(6.13)$, for superior a uma tolerância $T O L_{\text {erro }}$ adotada para o problema. A determinação das regiões que devem ser enriquecidas é conduzida pelos indicadores de erro, $\tilde{\mathcal{E}}_{\mathcal{K}},(6.45)$, ou $\tilde{\mathcal{E}}_{\omega_{j}},(6.47)$, que podem estar vinculados aos elementos ou às nuvens respectivamente. Define-se um parâmetro, $v$, que caracteriza a velocidade de convergência do procedimento adaptativo. Primeiramente, calcula-se o indicador de maior valor, $\tilde{\mathcal{E}}_{\text {max }}$. Determina-se, então, que se realize o refinamento em todas as regiões que apresentarem os indicadores superiores a $v \tilde{\mathcal{E}}_{\max }{ }^{4}$. Dessa forma, se uma determinada região tem a aproximação caracterizada pelo grau polinomial $p$, o refinamento, caso seja necessário, deve ser tal que a nova aproximação seja de ordem $p+i_{p}$, sendo $i_{p}$ um

\footnotetext{
${ }^{4} \mathrm{O}$ parâmetro $v$ é responsável pela velocidade da convergência do procedimento adaptativo. Quanto menor $v$ maior o número de regiões refinadas em cada passo. Como conseqüência, chega-se à tolerância admitida para o erro com um menor número de passos mas a aproximação final fica mais distante da ótima, DUARTE (1991)
} 
valor pré-estabelecido. Por outro lado, se $p$ for igual a um valor máximo $p_{\max }$, adotado para a análise adaptativa, a respectiva região não deve ser refinada. Esta estratégia visa limitar o grau $p$ da aproximação para que esse não aumente indefinidamente. Se todas as regiões determinadas pelo indicador para serem refinadas já apresentarem $p=p_{\max }$, então é necessário reduzir o parâmetro $v$. Dessa forma o processo adaptativo prossegue, incrementando a aproximação de novas regiões, e possibilitando a redução do erro global. Caso não existam outras regiões disponíveis para o refinamento, ou seja, se em todas elas $p=p_{\max }$, não há como se reduzir a norma energia do erro estimado, através do refinamento polinomial, para a malha de elementos adotada. O algoritmo é, portanto, interrompido. Para o controle desse processo são empregados o contador $i_{\text {fim }}$ e a variável $n_{v}$, conforme é mostrado nos algoritmos 6.1 e 6.2.

Na descrição acima, optou-se por usar, propositadamente, o termo "regiões" para se definir como o refinamento é conduzido. Dessa forma, a descrição dada permanece válida para as duas estratégias esquematizadas nos algoritmos 6.1, e 6.2, em que o procedimento adaptativo é baseado, exclusivamente, em indicadores associados às nuvens e aos elementos respectivamente. É preciso que se entenda que o cálculo do estimador global, em ambos os algoritmos, permanece sendo realizado a partir dos indicadores locais em elementos, expressão (6.46). No algoritmo 6.1, entretanto, os indicadores em elementos são utilizados, também, para se definir os indicadores em nuvens, $\tilde{\mathcal{E}}_{\omega_{j}}$ através da expressão (6.47). Como visto na seção 2.5.1, o enriquecimento da aproximação no MEFG é definido em cada nuvem e não mais nos elementos, como ocorre com o MEF. Por essa razão, é natural que se utilize como indicador de erro para as $N$ nuvens $\omega_{j}$ que aproximam o domínio $\Omega$, a medida $\tilde{\mathcal{E}}_{\omega_{j}}$. É esse o esquema adotado, portanto, no algoritmo 6.1 .

Por outro lado, ainda que o refinamento continue sendo realizado para as nuvens, é o indicador de erro do elemento, $\tilde{\mathcal{E}}_{\mathcal{K}}$, que conduz o processo adaptativo no Algoritmo 6.2. Dessa forma, se em um determinado elemento $\mathcal{K}$ tem-se $\tilde{\mathcal{E}}_{\mathcal{K}}>v \tilde{\mathcal{E}}_{\max }$, as quatro nuvens associadas aos vértices do elemento devem ser enriquecidas. Tal procedimento precisa, contudo, que seja restringido para evitar que combinações de indicativos de refinamento entre elementos vizinhos produza um enriquecimento desnecessariamente elevado. Para isso, define-se o controlador $p_{\mathcal{K}}$, que armazena o grau polinomial mais baixo definido pelas funções associadas aos nós do elemento $\mathcal{K}$. Nenhuma nuvem de $\mathcal{K}$, cuja função de aproximação já tenha alcançado ordem superior a $p_{\mathcal{K}}$, pode ser novamente enriquecida. Dessa forma, em um dado elemento, apenas 


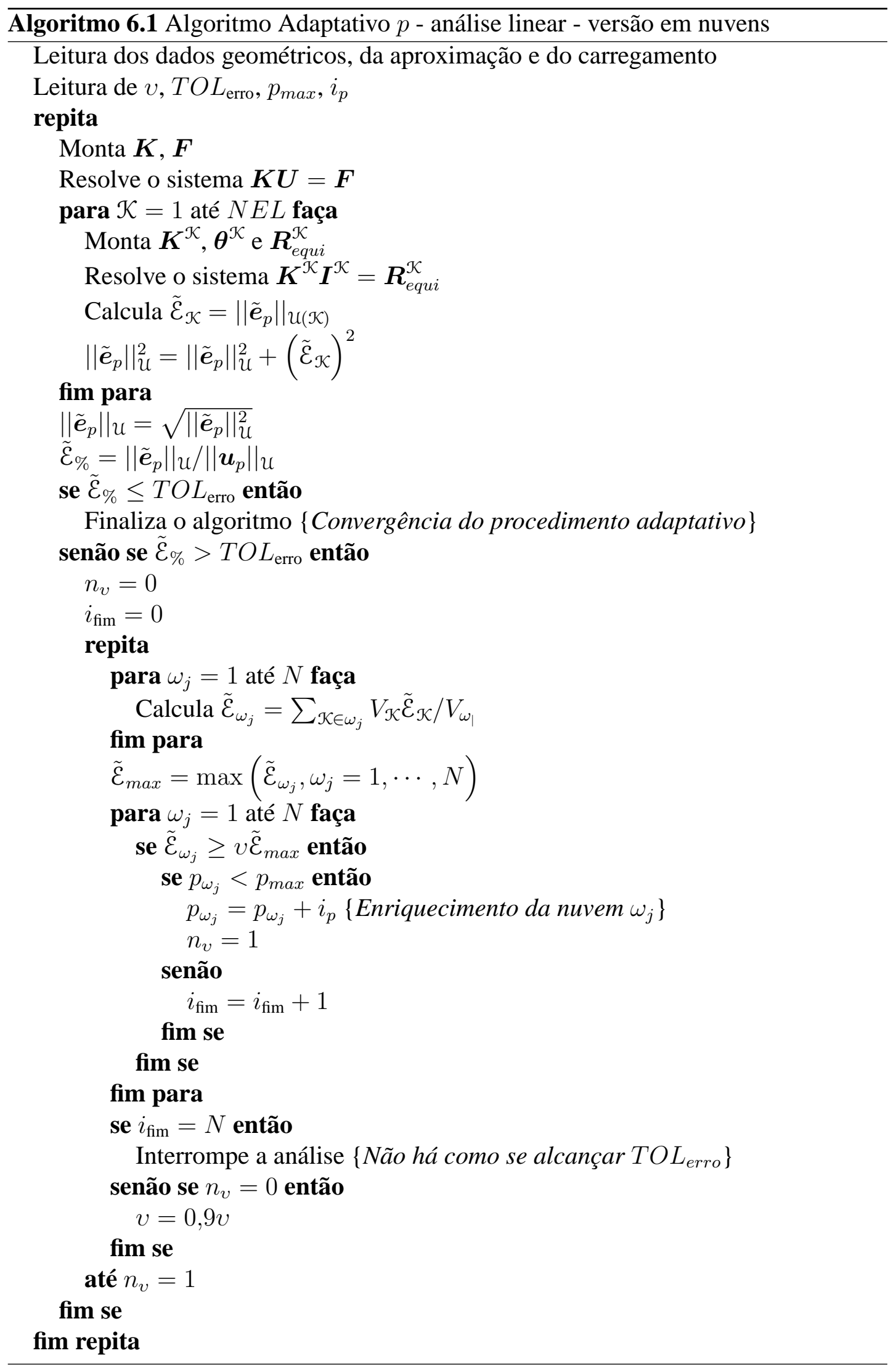




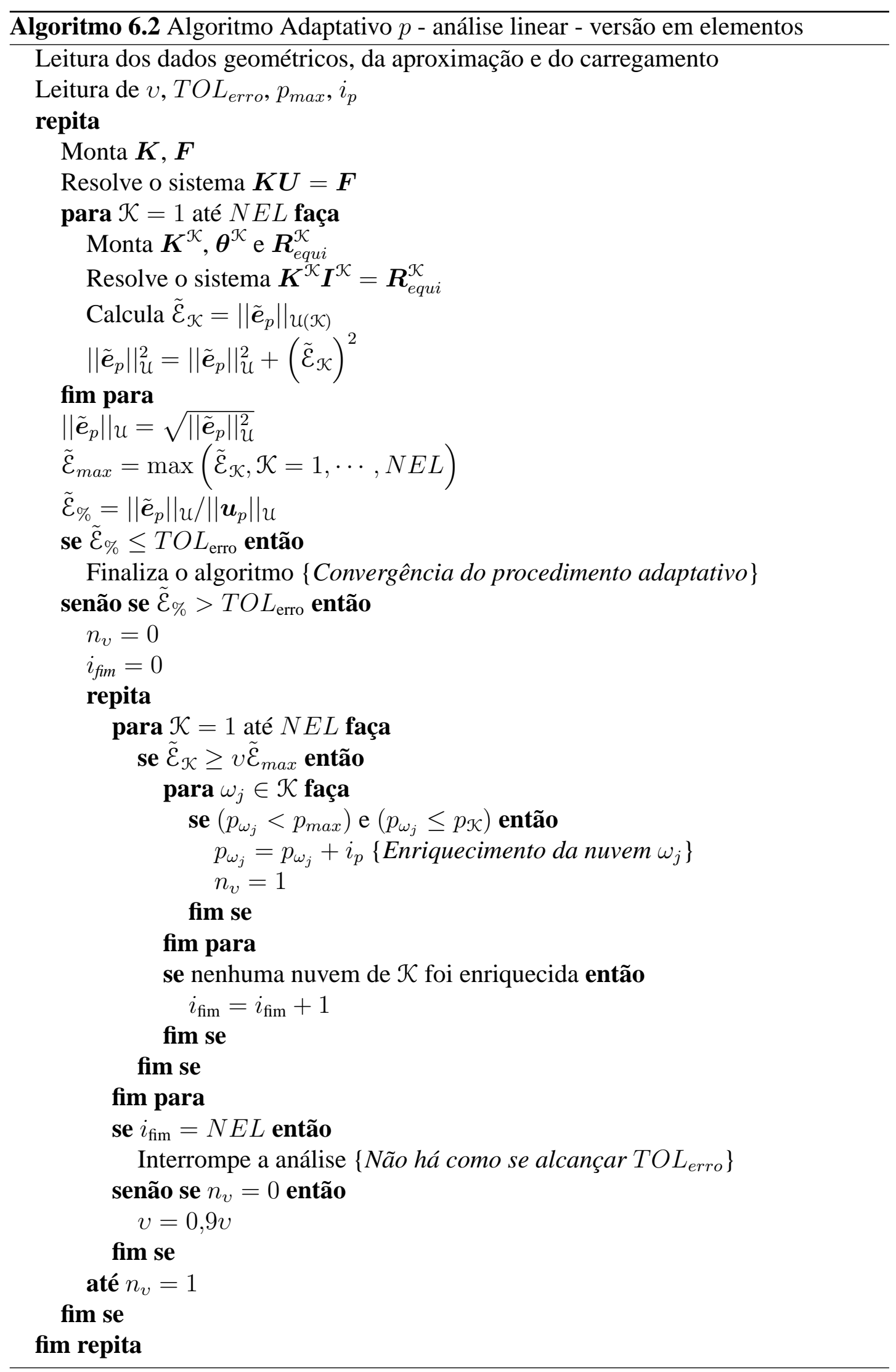


as nuvens de grau polinomial mais baixo são enriquecidas. Se no elemento vizinho um novo enriquecimento for exigido, aquelas nuvens anteriormente enriquecidas já se encontram com aproximação de grau superior ao $p_{\mathcal{K}}$ do presente elemento. Evita-se, assim, que a mesma nuvem seja enriquecida repetidamente para cada verificação por elemento.

\subsection{Exemplos Numéricos}

Nas duas seções seguintes, são apresentados dois exemplos numéricos, utilizados para se averiguar a implementação do estimador de erro e do procedimento adaptativo na abordagem do MEFG. Os problemas selecionados foram introduzidos no capítulo 3. Em todas as análises foi utilizado o MRE com o equilíbrio imposto pela estratégia de Ladevèze \& Maunder, seção 6.4. Os sistemas de equações (3.23) e (6.62) foram resolvidos pelo procedimento de Babǔska adotando-se $T O L=10^{-10} \mathrm{e} \epsilon=10^{-12}$.

O objetivo principal do primeiro exemplo consiste em mostrar a boa qualidade do erro estimado nos níveis local e global. O problema (6.33) é resolvido considerandose não apenas o espaço $X_{p+1}^{0}(\mathcal{K})$ mas também $X_{p+1, p+2}^{0}(\mathcal{K})$ que contém o primeiro e inclui novas funções de forma do MEFG utilizadas na representação de polinômios de grau $p+2$. Estes dois espaços são novamente empregados para estimar o erro no segundo exemplo. Além disso, são também apresentados os resultados para as análises adaptativas realizadas segundo os algoritmos 6.1 e 6.2. Não se pretende aqui estabelecer conclusões adicionais às encontradas na literatura do MEF no contexto de estimadores de erro. A finalidade é mostrar a validade das estratégias de erro e como estas podem ser empregadas na abordagem do MEFG, salientando-se suas peculiaridades e influência nos resultados das análises numéricas.

\subsubsection{Viga Engastada}

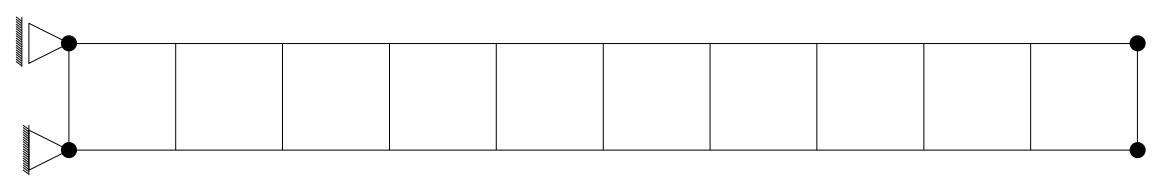

Figura 6.3: Malha formada por 10 elementos regulares quadrangulares 
O problema da viga engastada da seção 3.2.1 é aproximado pela malha de elementos apresentada na Figura 6.3. Recorda-se, que os vínculos utilizados servem somente para eliminar os movimentos de corpo rígido pois, como já foi observado naquela seção, a estrutura é equilibrada pelas condições de contorno naturais. A aproximação utilizada é linear ( $p=1)$, não havendo, portanto, funções enriquecidas.

$\mathrm{Na}$ seção 6.3, o espaço utilizado para a aproximação de Galerkin do problema (6.33) foi definido de forma genérica como $X_{p+1}^{0}(\mathcal{K})$. O índice $p+1$ significa que as funções presentes nesse espaço representam polinômios de grau superior àqueles representados pelas funções da aproximação $\boldsymbol{u}_{p}$. Teoricamente, quanto maior a dimensão do espaço das funções bolha melhor deve ser a estimativa do erro. Normalmente, entretanto, são usados espaços cujas funções reproduzem polinômios de um grau a mais apenas, daí a representação $X_{p+1}^{0}(\mathcal{K})$. Em DUARTE (1991) sugere-se a ampliação desse espaço para dois graus a mais, ou seja:

$$
\begin{gathered}
\left\{\boldsymbol{v}_{p+1, p+2}^{0}(\mathcal{K})=\left\{\begin{array}{c}
0 \\
\Pi_{p+1, p+2}\left(\boldsymbol{v}_{p+1, p+2}^{0}\right)=0, \boldsymbol{v}_{p+1, p+2}^{0}(\mathcal{K}) \subset \mathcal{H}^{1} ;
\end{array}\right.\right. \\
\left.\quad \text { em } \Gamma_{D}\right\}
\end{gathered}
$$

Possibilita-se, assim, que novas componentes do erro, que não seriam consideradas no espaço $X_{p+1}^{0}(\mathcal{K})$ venham a ser aproximadas.

Neste exemplo, os dois espaços $X_{p+1}^{0}(\mathcal{K})$ e $X_{p+1, p+2}^{0}(\mathcal{K})$ são empregados, definindose os dois estimadores descritos a seguir:

MRE $_{2}$ : o procedimento é o mesmo descrito na seção 6.3, em que o problema (6.33) é construído para o espaço $X_{2}^{0}$, ou seja, uma ordem superior à empregada para análise. As funções bolha empregadas em cada nó $\boldsymbol{x}_{j}$ são portanto:

$$
\left(\boldsymbol{\Phi}_{2}^{0}\right)_{j}^{T}=\left[\begin{array}{cccc}
\mathcal{N}_{j}\left(\frac{x-x_{j}}{h_{j}}\right) & 0 & \mathcal{N}_{j}\left(\frac{y-y_{j}}{h_{j}}\right) & 0 \\
0 & \mathcal{N}_{j}\left(\frac{x-x_{j}}{h_{j}}\right) & 0 & \mathcal{N}_{j}\left(\frac{y-y_{j}}{h_{j}}\right)
\end{array}\right]
$$

$\mathbf{M R E}_{\mathbf{2 , 3}}$ : as funções locais do erro são aproximadas no espaço $X_{2,3}^{0}$, ou seja, uma e duas ordens acima da empregada na fase de análise. Sendo assim, às funções bolha de (6.65) são acrescentados mais alguns termos, resultando em: 


$$
\begin{aligned}
& \left(\Phi_{2,3}^{0}\right)_{j}^{T}=\left[\begin{array}{cccc}
\mathcal{N}_{j}\left(\frac{x-x_{j}}{h_{j}}\right) & 0 & \mathcal{N}_{j}\left(\frac{y-y_{j}}{h_{j}}\right) & 0 \\
0 & \mathcal{N}_{j}\left(\frac{x-x_{j}}{h_{j}}\right) & 0 & \mathcal{N}_{j}\left(\frac{y-y_{j}}{h_{j}}\right)
\end{array}\right. \\
& \left.\begin{array}{cccc}
\mathcal{N}_{j}\left(\frac{x-x_{j}}{h_{j}}\right)^{2} & 0 & \mathcal{N}_{j}\left(\frac{y-y_{j}}{h_{j}}\right)^{2} & 0 \\
0 & \mathcal{N}_{j}\left(\frac{x-x_{j}}{h_{j}}\right)^{2} & 0 & \mathcal{N}_{j}\left(\frac{y-y_{j}}{h_{j}}\right)^{2}
\end{array}\right]
\end{aligned}
$$

Como a solução analítica é conhecida, (3.28) e (3.29), torna-se possível determinar, os valores global e para cada elemento $\mathcal{K}$ das normas exatas $\left\|\boldsymbol{e}_{p}\right\|_{u}$ e $\left\|\boldsymbol{e}_{p}\right\|_{u(\mathcal{K})}$ respectivamente. Estes valores são utilizados no cálculo do índice de efetividade do erro $\theta$ para toda a estrutura, (6.16), e por elemento, (6.17). Os resultados, para os dois estimadores de erro, são exibidos na Figura 6.4.

No que se refere à integração numérica, existe a possibilidade de serem adotadas diferentes ordens de quadratura para a construção do sistema (3.23), na fase de análise, e do sistema (6.62) na fase de estimativa do erro. Optou-se, entretanto, por empregar $N G=3 \times 3$ pontos de Gauss-Legendre, correspondente ao número necessário para uma integração exata na fase de estimativa do erro.

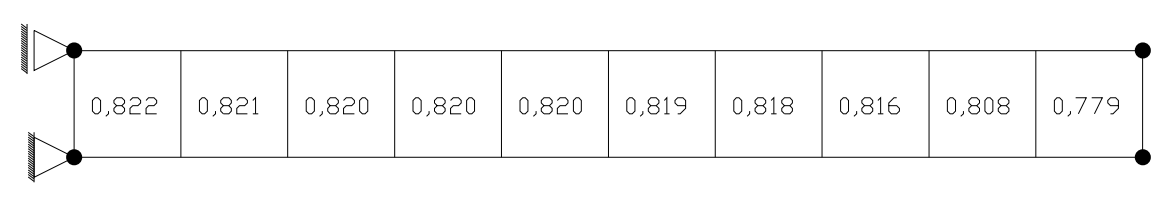

(a) $\mathrm{MRE}_{2}$ - Valor global $\theta=0,820$

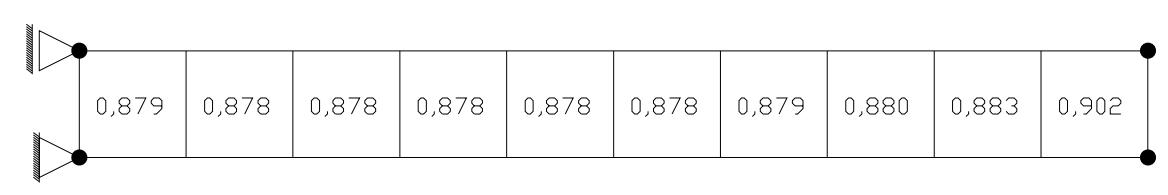

(b) $\mathrm{MRE}_{2,3}$ - Valor global $\theta=0,878$

Figura 6.4: Índices locais de efetividade da malha da Figura 6.3, para cada elemento

Analisando-se os resultados, Figura 6.4, nota-se que a norma do erro foi melhor representada pelo estimador obtido através do $\mathrm{MRE}_{2,3}$. Este fato já deveria ser espe- 
rado, uma vez que se ampliou o espaço para a aproximação do erro. A diferença entre as duas estimativas, entretanto, é pequena o que possibilita concluir que a parcela mais significativa do erro é quadrática, apenas um grau a mais do que aproximação linear da solução. Por outro lado, no elemento localizado na extremidade direita da viga, em que se registra a maior diferença entre os índices de efetividade, a componente cúbica do erro adquire maior importância.

Outro fator importante a ser considerado na definição de qual espaço aproximar as funções locais de erro corresponde ao custo computacional envolvido na solução do problema (6.33). Quanto maior a dimensão do espaço de funções bolha, maior o número de graus de liberdade para se descrever a aproximação das funções de erro e, conseqüientemente, maior o custo computacional exigido. Das expressões (6.65) e (6.66), observa-se que ocorre um acréscimo de duas funções por nó. Em um processo $p$-adaptativo, em que elevadas ordens polinomiais são empregadas na aproximação da solução, esse acréscimo pode ser ainda maior. De $X_{4}^{0}(\mathcal{K})$ para $X_{4,5}^{0}(\mathcal{K})$, por exemplo, pode-se mostrar que são 4 funções a mais e de $X_{5}^{0}(\mathcal{K})$ para $X_{5,6}^{0}(\mathcal{K})$ são introduzidas 7 novas funções. Para um problema com um elevado número de elementos o custo computacional do estimador de erro pode ser, portanto, bastante inflacionado pela projeção do erro em espaços do tipo $X_{p+1, p+2}^{0}(\mathcal{K})$.

Resumindo, a diferença entre as duas estimativas de erro, para o exemplo apresentado, não é tão grande a ponto de prejudicar, por exemplo, uma análise adaptativa. Torna-se, então, mais interessante que espaços de menor dimensão como $X_{p+1}^{0}(\mathcal{K})$ sejam empregados. Deve-se, entretanto, ter sempre em consideração que os estimadores obtidos com espaços $X_{p+1, p+2}^{0}(\mathcal{K})$ são mais robustos por serem menos passíveis de falhar em problemas cuja a solução apresente caráter predominantemente par ou ímpar, DUARTE (1991). Por estas razões na escolha de qual espaço aproximar o erro, é sempre importante que sejam levados em conta a qualidade da estimativa e o custo computacional para obtê-la.

Um outro experimento numérico realizado consiste na análise do estimador $\mathrm{MRE}_{2}$ com relação à convergência do índice de efetividade. Para isso, foi utilizada uma seqüência de três malhas "aninhadas". Tendo-se como original a malha da Figura 6.3, cada malha subseqüente é obtida dividindo-se em quatro os elementos da malha anterior. Sendo assim, o erro estimado global foi calculado para malhas com 10, 40 e 160 elementos e os resultados para os correspondentes índices de efetividade, (6.16), são apresentados na Figura 6.5. O refinamento é caracterizadas pela dimensão $h$, cor- 


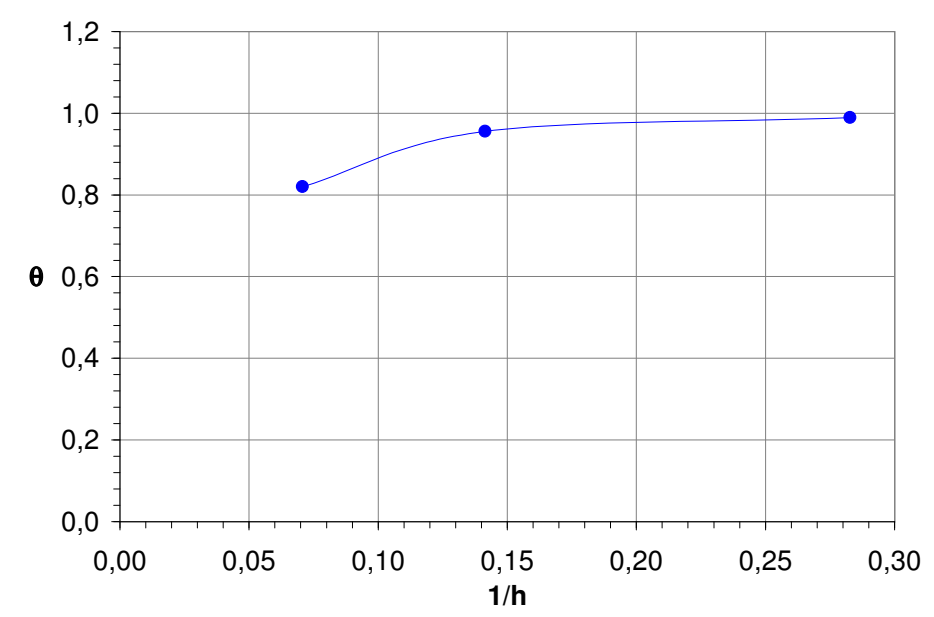

Figura 6.5: Índice de efetividade de $\mathrm{MRE}_{2}$ para uma seqüencia de 3 malhas aninhadas

respondente ao raio das nuvens empregadas, ou seja, à diagonal do elemento quadrangular. Pelo comportamento da curva $\theta \times 1 / h$ conclui-se que, para esse problema, o estimador de erro $\mathrm{MRE}_{2}$ é assintoticamente exato.

Como último teste, os dois métodos $\mathrm{MRE}_{2} \mathrm{e} \mathrm{MRE}_{2,3}$ foram avaliados com uma malha em que os elementos se encontram bastante distorcidos, Figura 6.6(a). As mesmas considerações feitas anteriormente, para a análise e estimativa de erro, são adotadas, exceto com relação à integração numérica. Devido à distorção da malha o jacobiano do mapeamento entre os elementos mestre e real torna-se racional e, por isso, as integrações numéricas realizadas no interior dos elementos exigem um maior número de pontos da quadratura. Outro fator que deve ser comentado refere-se ao equilíbrio dos resíduos. Caso a ordem da quadratura na fase de análise seja diferente daquela usada na fase de estimativa de erro a integral do jacobiano não fornecerá o mesmo valor. Como consequiência, o equilíbrio nodal deixa de ser averiguado na fase de estimativa de erro, pois esse foi imposto durante a análise, com base nas integrações numéricas então realizadas. O método de equilíbrio de Ladevèze \& Maunder falha, portanto, no momento de se empregar o diagrama da Figura 6.2(b). Para se evitar tal problema procurou-se usar o mesmo número de pontos na integração numérica para as fases de análise e de estimativa. No problema estudado, foram adotados $N G=5 \times 5$, para ambos os $\mathrm{MRE}_{2}$ e $\mathrm{MRE}_{2,3}$.

Os índices de efetividade para os dois estimadores são apresentados para cada elemento da malha nas Figuras 6.6(b) e 6.6(c). Observa-se que os os índices de efeti- 


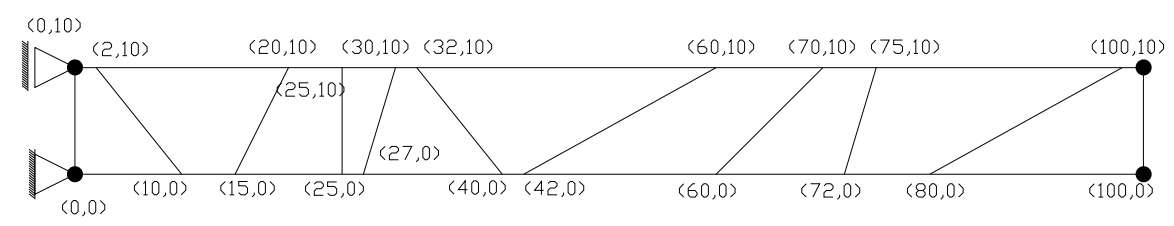

(a) Malha adotada

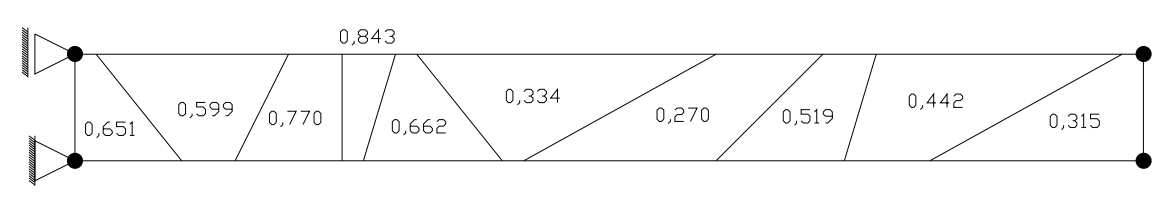

(b) $\mathrm{MRE}_{2}$ - Valor global $\theta=0,558$

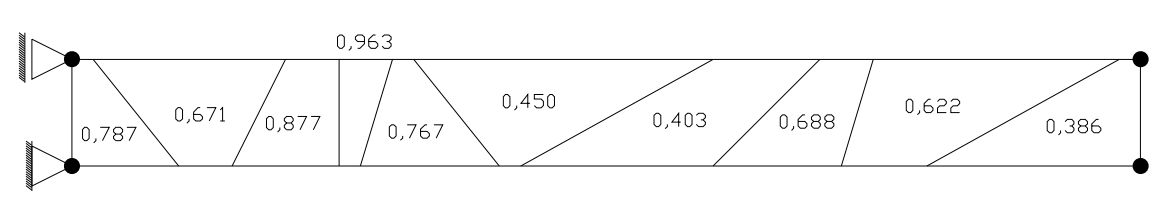

(c) $\mathrm{MRE}_{2,3}$ - Valor global $\theta=0,663$

Figura 6.6: Índices locais de efetividade - Malha de elementos distorcidos

vidade do elemento e global são inferiores aos encontrados para as análises anteriores. A explicação para tais resultados está no erro de aproximação, que para a análise com elementos distorcidos tem $\tilde{\varepsilon}_{\%}=75,18 \%$, bem superior ao encontrado na análise com elementos regulares, $\tilde{\mathcal{E}}_{\%}=57,13 \%$. Caso os elementos da malha da Figura 6.6 sejam divididos ao meio e nova análise seja realizada, obtém-se um índice de efetividade global $\theta=0,634$ para o $\mathrm{MRE}_{2}$ e $\theta=0,700$ para o $\mathrm{MRE}_{2,3}$, indicando uma melhora na estimativa de erro, como deve ocorrer para um estimador assintoticamente exato. No que se refere à comparação entre as estimativas com o $\mathrm{MRE}_{2}$ e o $\mathrm{MRE}_{2,3}$, permanecem válidas as conclusões extraídas dos resultados obtidos com elementos regulares.

\subsubsection{Chapa com Orifício}

Neste exemplo, considera-se, novamente, o problema da seção 3.2.2. Na Figura 6.7, encontra-se representada a malha de elementos adotada. Dois experimentos foram realizados. No primeiro deles, repete-se o estudo com os estimadores do $\mathrm{MRE}_{2}$ e do $\mathrm{MRE}_{2,3}$, realizado na seção 6.5.1. Em todo o processo de análise e de estimativa de erro e para ambos os métodos, foram empregados $4 \times 4$ pontos de Gauss-Legendre 


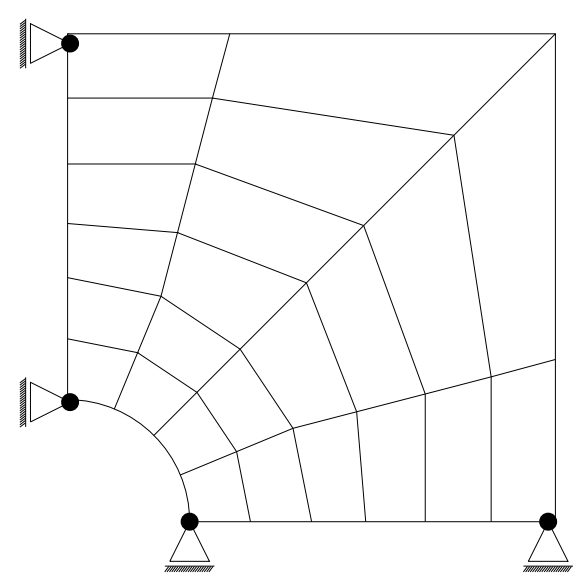

Figura 6.7: Malha de elementos adotada

por elemento. A Figura 6.8 apresenta os valores locais e globais do índice de efetividade dos estimadores de erro empregados. Observa-se que, da mesma forma que na seção 6.5.1, o estimador obtido com o $\mathrm{MRE}_{2,3}$ tem os melhores valores para $\theta$. Ainda assim, pode-se dizer que, para o $\mathrm{MRE}_{2}$ os resultados são, também, muito bons. Nos elementos que contornam o orifício, foram utilizadas as funções de mistura para se descrever as faces curvas. Por essa razão, o mapeamento entre os elementos mestre e real deixa de ser linear. Como conseqüência, a aplicação da estratégia de Ladevèze $\&$ Maunder não assegura que os dados para o vetor $\boldsymbol{R}_{\text {equi }}^{\mathcal{K}}$ verifiquem o equilíbrio do momento, conforme se discute no apêndice H para as expressões (H.8). Apesar disso, os indicadores de erro calculados para os elementos de face curva apresentam $\theta \approx 1$.

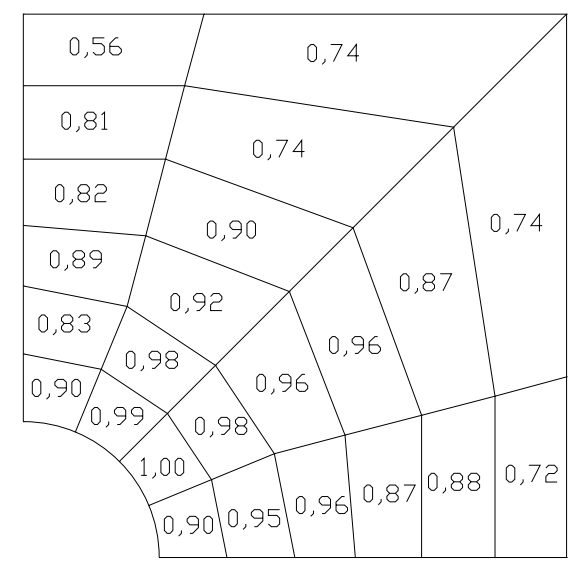

(a) $\mathrm{MRE}_{2}$ - Valor global $\theta=0,939$

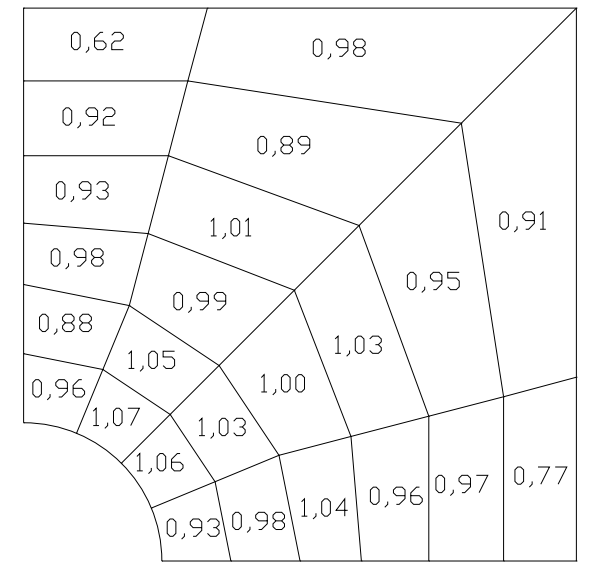

(b) $\mathrm{MRE}_{2,3}$ - Valor global $\theta=1,002$

Figura 6.8: Índices locais de efetividade - malha com aproximação linear 
O segundo experimento consiste em se aplicar o procedimento $p$ adaptativo, através dos dois algoritmos descritos na seção 6.4.2. Para a análise e estimativa foram feitas as seguintes considerações:

integração Numérica : para a análise e estimativa de erro correspondentes ao primeiro passo do procedimento adaptativo, correspondente à aproximação linear, foi adotado $\mathrm{NG}=4 \times 4$ pontos de Gauss-Legendre por elemento. Para os passos seguintes, em que a ordem polinomial das funções em cada nuvem varia conforme o refinamento exigido, o número de pontos da quadratura foi elevado de forma consistente com a aproximação construída nos respectivos elementos;

erro relativo almejado : definiu-se que o erro relativo estimado deve ser menor ou igual a $1 \%$, ou seja, $\mathcal{E}_{\%} \leq 1 \%\left(\right.$ TO $\left.L_{\text {erro }}=1 \%\right)$. Observa-se que, no lugar do erro relativo exato, $\mathcal{E}_{\%}$, emprega-se o valor estimado $\tilde{\mathcal{E}}_{\%}$, expressão (6.13);

velocidade de convergência : em DUARTE (1991) argumenta-se, com base no trabalho de LYRA (1988) em problemas de potencial, que $v$ deve ser superior a 0,4. Sendo assim, foi adotado $v=0,5$;

ordem polinomial máxima : para evitar problemas de condicionamento da matriz de rigidez, adotou-se $p_{\max }=8$;

método de estimativa de erro : foi empregado o MRE equilibrado com projeção do erro no espaço $X_{p+1}^{0}$, ou seja $\mathrm{MRE}_{p+1}$. Esta decisão baseia-se nos resultados obtidos para as estimativas de erro com o $\mathrm{MRE}_{2}$ e $\mathrm{MRE}_{2,3}$ que, para este problema, não se distanciaram muito uma da outra. Acredita-se, portanto, que a superioridade demonstrada por uma utilização do $\mathrm{MRE}_{p+1, p+2}$ seria prejudicada pelo elevado número de graus de liberdade exigido ao longo do refinamento adaptativo.

\begin{tabular}{|c|c|c|c|c|c|c|}
\hline \multirow{2}{*}{ Iteração } & \multicolumn{3}{|c|}{ Algoritmo 6.1 } & \multicolumn{3}{c|}{ Algoritmo 6.2 } \\
\cline { 2 - 7 } & $N G L$ & $\theta$ & $\varepsilon_{\%}$ & $N G L$ & $\theta$ & $\mathcal{E}_{\%}$ \\
\hline \hline 1 & 70 & 0,938 & $9,70 \%$ & 70 & 0,938 & $9,70 \%$ \\
\hline 2 & 114 & 0,930 & $5,32 \%$ & 118 & 0,933 & $4,98 \%$ \\
\hline 3 & 210 & 0,938 & $2,08 \%$ & 222 & 0,958 & $1,81 \%$ \\
\hline 4 & 286 & 0,970 & $1,00 \%$ & 354 & 0,917 & $0,49 \%$ \\
\hline
\end{tabular}

Tabela 6.1: Índices globais de efetividade para as iterações dos refinamentos adaptativos $\mathrm{MRE}_{p+1}$ 


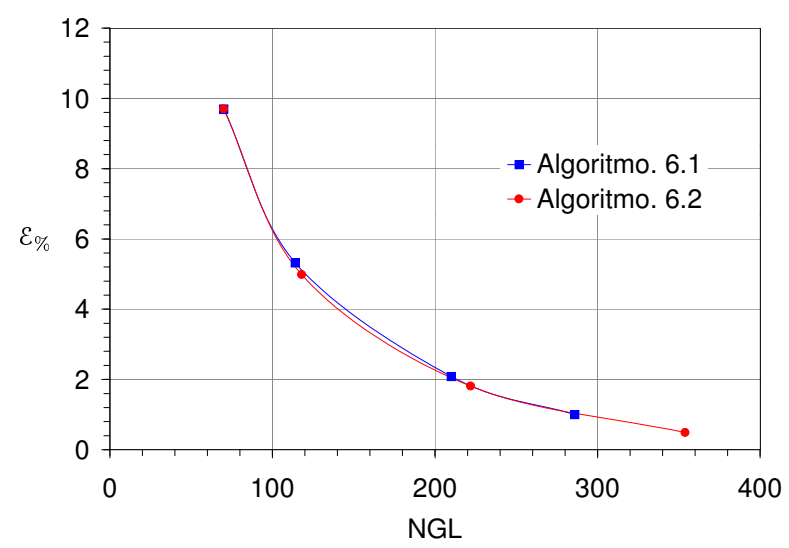

Figura 6.9: Erro relativo para as iterações dos refinamentos adaptativos

Na Tabela 6.1 encontram-se registrados o índice de efetividade, erro relativo exato e número de graus de liberdade (NGL) para cada passo das duas análises adaptativas realizadas. Nota-se que, em ambos os casos, o índice $\theta$ esteve sempre próximo da unidade. Na comparação entre as duas análises, utilizando-se o Algoritmo 6.2 chega-se a um nível de erro inferior ao obtido com o Algoritmo 6.1, porém com um $N G L$ maior. Para melhor entender como a análise adaptativa se comporta com relação aos dois algoritmos recorre-se ao gráfico da Figura 6.9, observando-se uma certa equivalência entre ambos as estratégias. Uma conclusão definitiva sobre qual o melhor algoritmo exige, na verdade, análises mais profundas, fundamentas em uma razoável quantidade de experimentos numéricos.

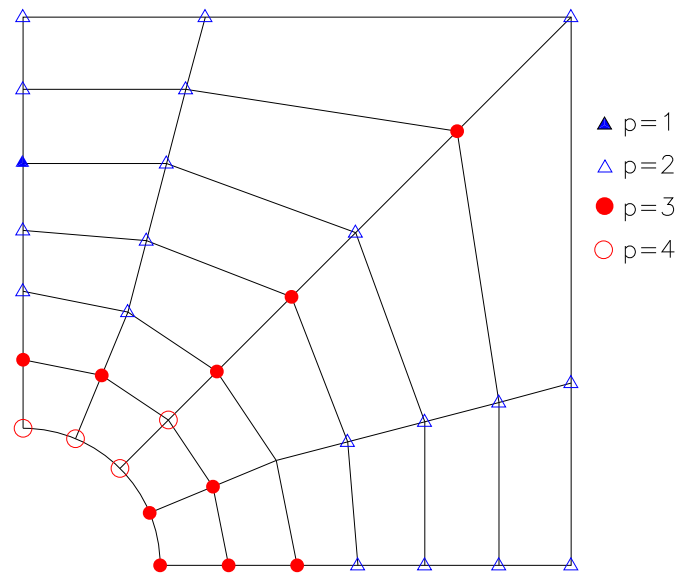

(a) Indicadores em nuvens, Algoritmo 6.1

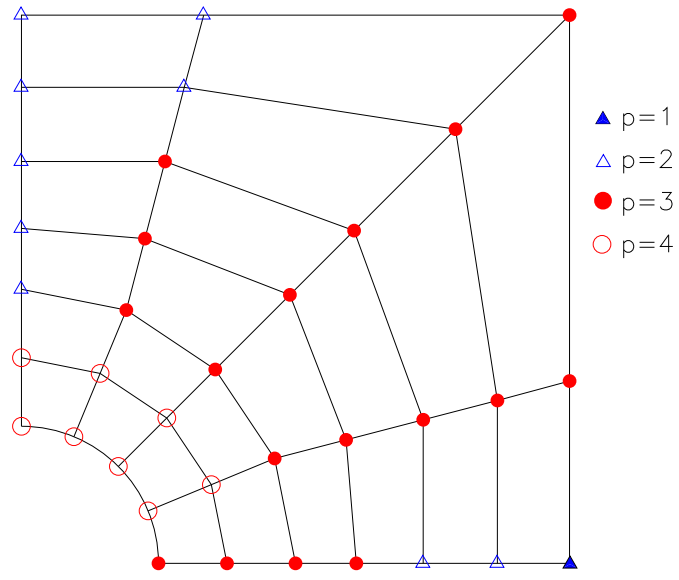

(b) Indicadores em elementos, Algoritmo 6.2

Figura 6.10: Resultado final do refinamento $p$-adaptativo, $\mathrm{MRE}_{p+1}$ 
Na Figura 6.10, estão representados os enriquecimentos das nuvens obtidos ao final das duas análises adaptativas. Uma pequena diferença é encontrada para as aproximações definidas pelos dois algoritmos. Com o algoritmo (6.1) o refinamento é realizado de maneira independente dos elementos. Explica-se, por isso, a maior variação entre os enriquecimentos de nuvens vizinhas.

\subsection{Medida de Erro em Análise Não-Linear}

Na seção 6.2.2, justificou-se o emprego do MRE por diversos fatores, entre eles o interesse em se aplicar o refinamento do tipo $p$, dada a facilidade com que esse é realizado no MEFG. Os resultados apresentados na seção (6.5) comprovaram a eficiência do estimador de erro e do procedimento adaptativo empregados, sob a abordagem do MEFG, para a análise numérica de problemas de elasticidade-linear. Para a análise dos problemas não-lineares apresentados neste trabalho, procurou-se aplicar o MEFG sob o mesmo enfoque dos problemas lineares, ou seja, construindo-se a solução aproximada por meio do enriquecimento polinomial das funções de PU. Sendo assim, é natural que o estimador de erro do problema não-linear continue sendo obtido pelo MRE. Para que isso possa ser realizado é necessário, contudo, que uma série de fatores sejam considerados.

Em análise não-linear, além do erro da aproximação em cada instante de tempo ${ }^{5}$ duas outras componentes de erro são introduzidas pelo processo de solução incrementaliterativo, PINTO (2000). A primeira delas é oriunda da divisão da história de carregamento em incrementos ou passos finitos. O equilíbrio das equações de governo deixa de ser assegurado ao longo de todo o carregamento, sendo verificado apenas ao final de cada passo. A outra componente é introduzida pela estratégia iterativa de correção do resíduo de forças, em razão da qual o equilíbrio é verificado apenas aproximadamente, dentro de uma certa tolerância admitida. Neste trabalho preocupa-se apenas com o erro da aproximação do MEFG, que se torna tão mais importante, em relação às outras componentes de erro mencionadas, quanto menores forem o passo de carga e a tolerância adotados. Estimativas do erro que levam em consideração as demais componentes podem ser encontradas em GALLIMARD; LADEVÈZE; PELLE (1996) e GALLIMARD; LADEVÈZE; PELLE (2000), em abordagem via MEF.

\footnotetext{
${ }^{5}$ Para ser mais exato além do erro de aproximação também estão presentes os erros da modelagem matemática, de arredondamento numérico, da integração numérica das matrizes e vetores do método numérico utilizado, da aproximação do domínio e da representação das condições de contorno
} 
Ainda relacionado ao significado do erro que se quer estimar, deve-se definir se esse é calculado para todo o processo de solução incremental ou se está associado a cada passo de carga isoladamente. Dessa forma, pode-se estabelecer se, após o refinamento adaptativo, é apenas o atual passo de carga que deve ser repetido ou se nova análise deve ser realizada.

Outro ponto importante refere-se ao emprego da norma energia que, em problemas lineares, está diretamente relacionada ao princípio de energia potencial mínima. Por outro lado, na formulação não-linear, outras medidas de erro podem ser melhor empregadas, como as normas obtidas da energia de dissipação ou da taxa de trabalho realizado, PERIĆ; YU; OWEN (1994).

Como adaptar o MRE para o problema não-linear resolvido pelo MEFG e se a medida de erro obtida corresponde realmente a um estimador são assuntos pertinentes à próxima seção. Além dos fatores considerados até aqui, são também discutidas outras questões, procurando-se estabelecer os limites de aplicação da técnica utilizada. Algumas simplificações importantes são realizadas, privando o estimador de suas garantias de convergência assintótica, mas permitindo sua utilização, sem grandes alterações com relação ao procedimento linear. A proposta, portanto, não é desenvolver um novo estimador para problemas não-lineares, mas utilizar a estratégia já implementada para o MEFG e empregada com sucesso em problemas lineares. O que se procura mostrar é que apesar das simplificações adotadas as medidas locais de erro ainda são suficientes para conduzir o refinamento polinomial adaptativo, possibilitando uma eficiente análise com o MEFG de problemas de propagação de dano.

\subsubsection{Estratégia de Estimativa do Erro}

Seja um problema com não-linearidade física resolvido através do procedimento da seção 5.3. Considera-se que o equilíbrio já esteja verificado até o passo $t$. Sendo assim, no passo $t+\Delta t$, o equilíbrio que se procura assegurar em um sentido generalizado equivale a:

$$
\begin{array}{rlccc}
\nabla^{T} \boldsymbol{\Sigma}\left({ }^{t+\Delta t} \boldsymbol{u}\right)+{ }^{t+\Delta t} \boldsymbol{b} & = & \mathbf{0} & \text { em } & \Omega \\
\boldsymbol{t}\left({ }^{t+\Delta t} \boldsymbol{u}\right) & ={ }^{t+\Delta t} \hat{\boldsymbol{t}} & \text { em } & \Gamma_{N} \\
{ }^{t+\Delta t} \boldsymbol{u} & ={ }^{t+\Delta t} \hat{\boldsymbol{u}} & \mathrm{em} & \Gamma_{D}
\end{array}
$$

onde:

- ${ }^{t+\Delta t} \boldsymbol{b}$ e ${ }^{t+\Delta t} \hat{\boldsymbol{t}}$ são as forças de volume e tensões de superfície no passo $t+\Delta t$;

- ${ }^{t+\Delta t} \boldsymbol{u}$ é a solução obtida ao final do passo $t+\Delta t$. 
- ${ }^{t+\Delta t} \hat{\boldsymbol{u}}$ é o deslocamento prescrito ao final do passo $t+\Delta t$

Considera-se, agora, uma aproximação da solução no passo $t+\Delta t$ representada por ${ }^{t+\Delta t} \boldsymbol{u}_{p} \in X_{p}$. Um problema análogo ao expresso em (6.21) pode, então, ser estabelecido:

$$
\begin{aligned}
& \text { Encontrar }{ }^{t+\Delta t} \boldsymbol{e}_{p}={ }^{t+\Delta t} \boldsymbol{u}-{ }^{t+\Delta t} \boldsymbol{u}_{p} \text { tal que: } \\
& \begin{aligned}
\nabla^{T}\left[\boldsymbol{\Sigma}\left({ }^{t+\Delta t} \boldsymbol{u}\right)-\boldsymbol{\Sigma}\left({ }^{t+\Delta t} \boldsymbol{u}_{p}\right)\right]+\boldsymbol{r}_{\Omega}\left({ }^{t+\Delta t} \boldsymbol{u}_{p}\right) & =\mathbf{0} \mathrm{em} \Omega \\
{ }^{t+\Delta t} \boldsymbol{e}_{p} & =\mathbf{0} \mathrm{em} \Gamma_{D} \\
\boldsymbol{t}\left({ }^{t+\Delta t} \boldsymbol{u}\right)-\boldsymbol{t}\left({ }^{t+\Delta t} \boldsymbol{u}_{p}\right) & =\boldsymbol{r}_{\Gamma}\left({ }^{t+\Delta t} \boldsymbol{u}_{p}\right) \mathrm{em} \Gamma_{N}
\end{aligned}
\end{aligned}
$$

onde são definidos:

$$
\begin{array}{lll}
\boldsymbol{r}_{\Omega}\left({ }^{t+\Delta t} \boldsymbol{u}_{p}\right) & =\nabla^{T} \boldsymbol{\Sigma}\left({ }^{t+\Delta t} \boldsymbol{u}_{p}\right)+{ }^{t+\Delta t} \boldsymbol{b} & \text { em } \Omega \\
\boldsymbol{r}_{\Gamma}\left({ }^{t+\Delta t} \boldsymbol{u}_{p}\right) & ={ }^{t+\Delta t} \hat{\boldsymbol{t}}-\boldsymbol{t}\left({ }^{t+\Delta t} \boldsymbol{u}_{p}\right) & \text { em } \Gamma_{N}
\end{array}
$$

Observa-se que devido à não-linearidade do problema, as propriedades do meio podem ser diferentes para a solução ${ }^{t+\Delta t} \boldsymbol{u}$ e sua aproximação ${ }^{t+\Delta t} \boldsymbol{u}_{p}$. O erro ${ }^{t+\Delta t} \boldsymbol{e}_{p}$ não é portanto, exclusivo da aproximação e, por isso, $\boldsymbol{\Sigma}\left({ }^{t+\Delta t} \boldsymbol{u}\right)-\boldsymbol{\Sigma}\left({ }^{t+\Delta t} \boldsymbol{u}_{p}\right) \neq \boldsymbol{\Sigma}\left({ }^{t+\Delta t} \boldsymbol{e}_{p}\right)$ e $\boldsymbol{t}\left({ }^{t+\Delta t} \boldsymbol{u}\right)-\boldsymbol{t}\left({ }^{t+\Delta t} \boldsymbol{u}_{p}\right) \neq \boldsymbol{t}\left({ }^{t+\Delta t} \boldsymbol{e}_{p}\right)$. Para que seja descrito apenas segundo ${ }^{t+\Delta t} \boldsymbol{e}_{p}, \mathrm{o}$ problema (6.68) deve ser modificado, fazendo-se desaparecer os termos em ${ }^{t+\Delta t} \boldsymbol{u}$. É com esse objetivo que, em CIRAK; RAMM (2000), utiliza-se da expansão de Taylor para as tensões em torno da solução ${ }^{t+\Delta t} \boldsymbol{u}_{p}$ :

$$
\begin{aligned}
\boldsymbol{\Sigma}\left({ }^{t+\Delta t} \boldsymbol{u}\right) & \left.=\boldsymbol{\Sigma}\left({ }^{t+\Delta t} \boldsymbol{u}_{p}\right)+\left.\frac{\partial \boldsymbol{\Sigma}(\boldsymbol{u})}{\partial \boldsymbol{E}}\right|_{t+\Delta t} \boldsymbol{E} \boldsymbol{u}_{p}{ }^{t+\Delta t} \boldsymbol{u}-{ }^{t+\Delta t} \boldsymbol{u}_{p}\right) \\
& +\left.\frac{1}{2} \frac{\partial^{2} \boldsymbol{\Sigma}(\boldsymbol{u})}{\partial \boldsymbol{E}^{2}}\right|_{t+\Delta t} \boldsymbol{E}^{2}\left({ }^{t+\Delta t} \boldsymbol{u}-{ }^{t+\Delta t} \boldsymbol{u}_{p}\right)+\cdots
\end{aligned}
$$

onde $\boldsymbol{E}$ é o tensor de deformações. Desprezando-se os termos de elevada ordem, a expressão acima pode ser representada pelos dois primeiros termos apenas. Dessa forma, ao final do passo $t+\Delta t$ o tensor de tensões pode ser aproximado em torno da solução ${ }^{t+\Delta t} \boldsymbol{u}_{p}$ como: 


$$
\boldsymbol{\Sigma}\left({ }^{t+\Delta t} \boldsymbol{u}\right)=\boldsymbol{\Sigma}\left({ }^{t+\Delta t} \boldsymbol{u}_{p}\right)+\left.\frac{\partial \boldsymbol{\Sigma}(\boldsymbol{u})}{\partial \boldsymbol{E}}\right|_{t+\Delta t} \boldsymbol{u}_{p} \underbrace{\boldsymbol{E} \underbrace{t+\Delta t} \boldsymbol{u}-{ }^{t+\Delta t} \boldsymbol{u}_{p})}_{{ }^{t+\Delta t} \boldsymbol{e}_{p}}
$$

onde $\partial \boldsymbol{\Sigma}(\boldsymbol{u}) /\left.\partial \boldsymbol{E}\right|_{t+\Delta t} \boldsymbol{u}_{p}$ representa a forma tangente exata da relação constitutiva para um campo de deslocamentos definido por ${ }^{t+\Delta t} \boldsymbol{u}_{p}$. Substituindo-se essa expressão em (6.68), tem-se um novo PVC estabelecido para a aproximação ${ }^{t+\Delta t} \boldsymbol{e}_{p}^{*}$ para o erro exato ${ }^{t+\Delta t} \boldsymbol{e}_{p}:$

$$
\begin{aligned}
\left.\boldsymbol{\nabla}^{T}\left[\left.\frac{\partial \boldsymbol{\Sigma}(\boldsymbol{u})}{\partial \boldsymbol{E}}\right|_{t+\Delta t} \boldsymbol{u}_{p}{ }^{t+\Delta t} \boldsymbol{e}_{p}^{*}\right)\right]+\boldsymbol{r}_{\Omega}\left({ }^{t+\Delta t} \boldsymbol{u}_{p}\right) & =\mathbf{0} \mathrm{em} \Omega \\
{ }^{t+\Delta t} \boldsymbol{e}_{p}^{*} & =\mathbf{0} \mathrm{em} \Gamma_{D} \\
{\left[\left.\frac{\partial \boldsymbol{\Sigma}(\boldsymbol{u})}{\partial \boldsymbol{E}}\right|_{t+\Delta t} \boldsymbol{u}_{p} \boldsymbol{E}\left({ }^{t+\Delta t} \boldsymbol{e}_{p}^{*}\right)\right] \boldsymbol{n} } & =\boldsymbol{r}_{\Gamma}\left({ }^{t+\Delta t} \boldsymbol{u}_{p}\right) \text { em } \Gamma_{N}
\end{aligned}
$$

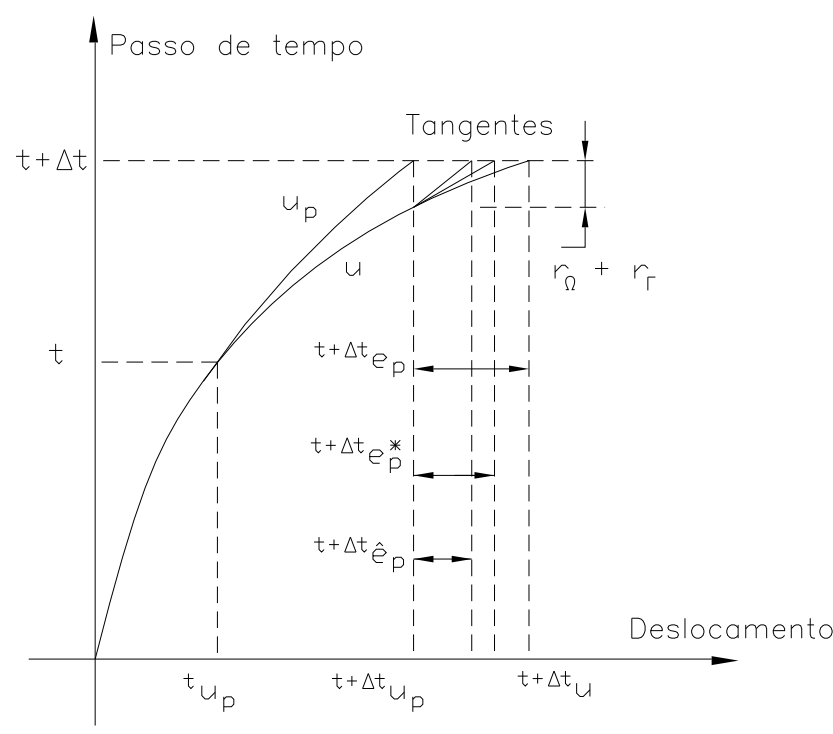

Figura 6.11: Interpretação geométrica para a estimativa do erro no caso uni-axial

Uma interpretação geométrica para o PVC acima, pode ser dada através da Figura 6.11, considerando-se o caso uni-axial. Resumindo, a tangente da curva $\boldsymbol{u}$ no ponto ${ }^{t+\Delta t} \boldsymbol{u}_{p}$ juntamente com o resíduo correspondente à incapacidade da solução aproximada ${ }^{t+\Delta t} \boldsymbol{u}_{p}$ em verificar o equilíbrio do problema (6.67) são utilizados para se calcular ${ }^{t+\Delta t} \boldsymbol{e}_{p}^{*}$. Caso estivessem sendo empregados todos os temos da série (6.70), a 
solução deste problema corresponderia ao valor exato do erro de aproximação, ou seja, ${ }^{t+\Delta t} \boldsymbol{e}_{p}$. Como é utilizada a forma linearizada (6.71), a solução ${ }^{t+\Delta t} \boldsymbol{e}_{p}^{*}$ corresponde a uma aproximação desse erro, tão mais próxima quanto melhor for a aproximação ${ }^{t+\Delta t} \boldsymbol{u}_{p}$. Em RHEINBOLDT (1985) mostra-se que, sob condições ideais, o erro ${ }^{t+\Delta t} \boldsymbol{e}_{p}^{*}$ é assintoticamente equivalente ao erro exato da aproximação ${ }^{t+\Delta t} \boldsymbol{e}_{p}$. Tais condições, apesar de não terem sido explicitadas nesse trabalho, referem-se ao comportamento da curva $u$ que deve ser sempre ascendente.

O problema assim formulado parte do pressuposto que se conheça o comportamento real do meio para que se possa utilizar a forma exata de $\partial \boldsymbol{\Sigma}(\boldsymbol{u}) /\left.\partial \boldsymbol{E}\right|_{t+\Delta t} \boldsymbol{u}_{p}$. Na realidade, a distribuição do dano em $t+\Delta t$ é função da flexibilidade da estrutura definida pelos graus de liberdade da aproximação ${ }^{t+\Delta t} \boldsymbol{u}_{p}$. Conhece-se, portanto, apenas a forma aproximada $\partial \boldsymbol{\Sigma}\left(\boldsymbol{u}_{p}\right) /\left.\partial \boldsymbol{E}\right|_{t+\Delta t} \boldsymbol{u}_{p}$ que, utilizada em (6.72), define outro PVC:

$$
\begin{aligned}
\boldsymbol{\nabla}^{T}\left[\left.\frac{\partial \boldsymbol{\Sigma}\left(\boldsymbol{u}_{p}\right)}{\partial \boldsymbol{E}}\right|_{t+\Delta t} \boldsymbol{E}\left(\boldsymbol{u}_{p}{ }^{t+\Delta t} \hat{\boldsymbol{e}}_{p}\right)\right]+\boldsymbol{r}_{\Omega}\left({ }^{t+\Delta t} \boldsymbol{u}_{p}\right) & =\mathbf{0} \mathrm{em} \Omega \\
{ }^{t+\Delta t} \hat{\boldsymbol{e}}_{p} & =\mathbf{0} \mathrm{em} \Gamma_{D} \\
{\left[\left.\frac{\partial \boldsymbol{\Sigma}\left(\boldsymbol{u}_{p}\right)}{\partial \boldsymbol{E}}\right|_{t+\Delta t} \boldsymbol{u}_{p} \boldsymbol{E}\left({ }^{t+\Delta t} \hat{\boldsymbol{e}}_{p}\right)\right] \boldsymbol{n} } & =\boldsymbol{r}_{\Gamma}\left({ }^{t+\Delta t} \boldsymbol{u}_{p}\right) \mathrm{em} \Gamma_{N}
\end{aligned}
$$

cuja solução ${ }^{t+\Delta t} \hat{\boldsymbol{e}}_{p}$ corresponde a uma estimativa para a função ${ }^{t+\Delta t} \boldsymbol{e}_{p}^{*}$, que por sua vez aproxima assintoticamente o erro ${ }^{t+\Delta t} \boldsymbol{e}_{p}$. Resta, então, inferir sobre qualidade de ${ }^{t+\Delta t} \hat{\boldsymbol{e}}_{p}$ como aproximação de ${ }^{t+\Delta t} \boldsymbol{e}_{p}$. Esta é uma questão relacionada com a nãolinearidade do problema analisado sendo, portanto, importante que dois aspectos da análise adaptativa sejam avaliados.

O primeiro deles refere-se ao tamanho dos passos para o procedimento de solução incremental. Considerando-se que a solução $\boldsymbol{u}_{p}$ reproduza exatamente $\boldsymbol{u}$ até o passo $t$, o erro ${ }^{t+\Delta t} e_{p}$ a ser encontrado é proveniente somente do passo $\Delta t$. Sendo esse passo suficientemente pequeno, a não-linearidade nas relações tensão-deformação é menos significativa. Dessa forma, espera-se que $\partial \boldsymbol{\Sigma}\left(\boldsymbol{u}_{p}\right) /\left.\partial \boldsymbol{E}\right|_{t+\Delta t} \boldsymbol{u}_{p}$ aproxime bem $\partial \boldsymbol{\Sigma}(\boldsymbol{u}) /\left.\partial \boldsymbol{E}\right|_{t+\Delta t} \boldsymbol{u}_{p}$ e, conseqüentemente, o problema (6.73) possa representar o PVC dado por (6.72).

O outro aspecto a ser considerado está relacionado ao controle do erro a cada passo. No parágrafo anterior admitiu-se a inexistência de erro entre as soluções exata e aproximada até o passo $t$. Esta hipótese é bastante severa e, obviamente, haverá 
sempre um erro de aproximação (sem levar em conta outras parcelas do erro com o de discretização no tempo). Pode-se, entretanto, chegar próximo a essa situação desde que o controle do erro seja realizado a cada passo, de maneira que se for necessário a solução seja refinada.

Considerando-se, então que:

- a solução aproximada ${ }^{t+\Delta t} \boldsymbol{u}_{p}$ tenha sido obtida através de um processo adaptativo em que o erro esteja sendo controlado a cada passo de tempo;

- o passo $\Delta t$ seja pequeno o suficiente para que $\partial \boldsymbol{\Sigma}\left(\boldsymbol{u}_{p}\right) /\left.\partial \boldsymbol{E}\right|_{t+\Delta t} \boldsymbol{u}_{p}$ seja uma boa aproximação para $\partial \boldsymbol{\Sigma}(\boldsymbol{u}) /\left.\partial \boldsymbol{E}\right|_{t+\Delta t} \boldsymbol{u}_{p} ;$

a seguinte forma variacional do problema (6.73), aproximada por Galerkin como na seção 6.3, pode ser empregada para se obter as funções indicadoras de erro para o passo $t+\Delta t$ :

$$
\begin{aligned}
& \text { Encontrar }{ }^{t+\Delta t} \tilde{\boldsymbol{e}}_{p} \in X_{p+1}^{0}(\mathcal{K}) \text { tal que: } \\
& \qquad \mathcal{B}_{\mathcal{K}}^{\mathrm{tg}}\left({ }^{t+\tilde{\Delta} t} \tilde{\boldsymbol{e}}_{p}, \boldsymbol{v}_{p+1}^{0}\right)=\mathcal{L}_{\mathcal{K}}\left(\boldsymbol{v}_{p+1}^{0}\right) \forall \boldsymbol{v}_{p+1}^{0} \in X_{p+1}^{0}(\mathcal{K})
\end{aligned}
$$

onde

$$
\begin{gathered}
\mathcal{B}_{\mathcal{K}}^{\text {tg }} \stackrel{\text { def }}{=} \iint_{\mathcal{K}} \varepsilon^{T}\left(\boldsymbol{v}_{p+1}^{0}\right) \boldsymbol{\sigma}^{\operatorname{tg}}\left({ }^{t+\Delta t} \tilde{\boldsymbol{e}}_{p}\right) l_{z} d x d y \\
\mathcal{L}_{\mathcal{K}}\left(\boldsymbol{v}_{p+1}^{0}\right) \stackrel{\text { def }}{=} \iint_{\mathcal{K}}\left(\boldsymbol{v}_{p+1}^{0}\right)^{T}\left({ }^{t+\Delta t} \boldsymbol{b}\right) l_{z} d x d y-\mathcal{B}_{\mathcal{K}}\left({ }^{t+\Delta t} \boldsymbol{u}_{p}, \boldsymbol{v}_{p+1}^{0}\right) \\
+\int_{\partial \mathcal{K} \cap \Gamma_{N}}\left(\boldsymbol{v}_{p+1}^{0}\right)^{T}\left({ }^{t+\Delta t} \hat{\boldsymbol{t}}\right) l_{z} d s+\int_{\partial \mathcal{K} \backslash \partial \Omega}\left(\boldsymbol{v}_{p+1}^{0}\right)^{T}\left\langle\boldsymbol{t}\left({ }^{t+\Delta t} \boldsymbol{u}_{p}\right)\right\rangle_{m} l_{z} d s
\end{gathered}
$$

Nota-se que está sendo empregada na (6.75) a forma vetorial compacta $\sigma$ do tensor de tensões $\Sigma$, definida a partir de (6.71), na forma tangente, como:

$$
\boldsymbol{\sigma}^{\operatorname{tg}}\left({ }^{t+\Delta t} \tilde{\boldsymbol{e}}_{p}\right)=\left.\frac{\partial \boldsymbol{\sigma}\left(\boldsymbol{u}_{p}\right)}{\partial \boldsymbol{\varepsilon}}\right|_{t+\Delta t} \boldsymbol{u}_{p}\left({ }^{t+\Delta t} \tilde{\boldsymbol{e}}_{p}\right)
$$

A medida global de erro utilizada como estimador é obtida através dos valores locais da norma energia, como em (6.46): 


$$
\left\|^{t+\Delta t} \tilde{\boldsymbol{e}}_{p}\right\|_{u}=\sqrt{\sum_{\mathcal{K} \in \Omega} \tilde{\mathcal{E}}_{\mathcal{K}}^{2}}
$$

onde para cada elemento tem-se:

$$
\tilde{\mathcal{E}}_{\mathcal{K}} \stackrel{\text { def }}{=}\left\|{ }^{t+\Delta t} \tilde{\boldsymbol{e}}_{p}\right\| u(\mathcal{K})=\left[\mathcal{B}_{\mathcal{K}}^{\text {tg }}\left({ }^{t+\Delta t} \tilde{\boldsymbol{e}}_{p},{ }^{t+\Delta t} \tilde{\boldsymbol{e}}_{p}\right)\right]^{1 / 2}
$$

As funções $\tilde{\boldsymbol{e}}_{p}$ determinadas para cada elemento são, portanto, representações, da função $\hat{\boldsymbol{e}}_{p}$ que, dependendo das condições da análise, podem ser ou não boas aproximações locais do erro $\boldsymbol{e}_{p}$.

Finalmente, o sistema de equações para as funções indicadoras de erro do elemento $\mathcal{K}$ no passo $t+\Delta t$, pode ser definido de maneira análoga ao sistema equilibrado (6.62), como:

$$
{ }^{t+\Delta t} \boldsymbol{K}_{\mathrm{er}}^{\mathcal{K}}{ }^{t+\Delta t} \boldsymbol{I}^{\mathcal{K}}={ }^{t+\Delta t} \boldsymbol{R}_{\mathrm{equi}}^{\mathcal{K}}
$$

onde são definidos:

- funções indicadoras do erro:

$$
{ }^{t+\Delta t} \tilde{\boldsymbol{e}}_{p}=\left(\boldsymbol{\Phi}_{p+1}^{0}\right)^{T t+\Delta t} \boldsymbol{I}
$$

- matriz de rigidez:

$$
{ }^{t+\Delta t} \boldsymbol{K}_{\mathrm{er}}^{\mathcal{K}}=\iint_{\mathcal{K}}\left(\boldsymbol{B}_{p+1}^{0}\right)^{T t+\Delta t} \boldsymbol{C}_{\mathrm{tg}} \boldsymbol{B}_{p+1}^{0} l_{z} d x d y
$$

- vetor de forças residuais nodais generalizadas

$$
\begin{aligned}
{ }^{t+\Delta t} \boldsymbol{R}_{\text {equi }}^{\mathcal{K}} & =\iint_{\mathcal{K}}\left(\Phi_{p+1}^{0}\right)^{T t+\Delta t} \boldsymbol{b} l_{z} d x d y-{ }^{t+\Delta t} \boldsymbol{K}^{\mathcal{K}}{ }^{t+\Delta t} \boldsymbol{U} \\
& +\int_{\partial \mathcal{K} \cap \Gamma_{N}}\left(\Phi_{p+1}^{0}\right)^{T t+\Delta t} \hat{\boldsymbol{t}} l_{z} d s+\int_{\partial \mathcal{K} \backslash \partial \Omega}\left(\boldsymbol{\Phi}_{p+1}^{0}\right)^{T}\left\langle\boldsymbol{t}\left({ }^{t+\Delta t} \boldsymbol{u}_{p}\right)\right\rangle_{m} l_{z} d s \\
& +\int_{\partial \mathcal{K}}\left(\Phi_{p+1}^{0}\right)^{T+t+\Delta t} \boldsymbol{\theta}^{\mathcal{K}} l_{z} d s
\end{aligned}
$$


Observa-se que a matriz de rigidez ${ }^{t+\Delta t} \boldsymbol{K}_{\mathrm{er}}^{\mathcal{K}}$ encontra-se na forma tangente. Ao se empregar o modelo constitutivo de Mazars, a seguinte expressão para ${ }^{t+\Delta t} \boldsymbol{C}_{\mathrm{tg}}$ deve ser, portanto, usada em (6.82):

$$
{ }^{t+\Delta t} \boldsymbol{C}_{\mathrm{tg}}=\left[(1-D) \boldsymbol{C}_{0}-\boldsymbol{C}_{0} \varepsilon \mathcal{F}\left(\varepsilon_{e q}\right) \frac{\partial \varepsilon_{e q}}{\partial \varepsilon}\right]_{t+\Delta t}
$$

em que os termos presentes estão definidos no apêndice A.

Da mesma forma que para o caso linear, os dados para o problema de estimativa de erro também devem ser equilibrados. É com esse objetivo que em (6.83) são introduzidas as tensões de correção $\boldsymbol{\theta}^{\mathcal{K}}\left({ }^{t+\Delta t} \boldsymbol{u}_{p}\right)$ definida para o passo $t+\Delta t$.

\subsubsection{Algoritmo Adaptativo}

Uma maneira bastante simples de interpretar a medida de erro $\left\|^{t+\Delta t} \boldsymbol{e}_{p}\right\|_{u}$, definida na seção 6.6.1, está relacionada à incapacidade da aproximação ${ }^{t+\Delta t} \boldsymbol{u}_{p}$ em verificar as equações diferenciais do problema (6.67) ao final do passo $t+\Delta t$. Não estão incluídas, portanto, informações sobre o erro ao longo da história do carregamento ${ }^{6}$. De fato, obter-se um valor pequeno para a medida $\mid{ }^{t+\Delta t} \boldsymbol{e}_{p} \|_{u}$ significa, somente, que ao final do passo $t+\Delta t$ o problema (6.67) foi bem aproximado. Nenhuma garantia é fornecida com relação à resposta não-linear da estrutura até esse passo.

Não há, portanto, como associar a medida $\mid{ }^{t+\Delta t} \tilde{\boldsymbol{e}}_{p} \|$ com o erro no passo, ou até o passo $t+\Delta t$, mas somente com o erro ao final desse passo. Ainda que pareça retórica, toda essa discussão é de extrema importância, não somente para a compreensão da medida de erro empregada, como também para a definição do algoritmo adaptativo. Conforme o estimador esteja associado ao erro do passo ou até o passo, a análise, com a nova aproximação enriquecida segundo o refinamento adaptativo, deve ser repetida para o passo ou a partir do início do carregamento respectivamente. No caso da medida $\mid{ }^{t+\Delta t} \tilde{\boldsymbol{e}}_{p} \|_{u}$, qualquer uma destas alternativas podem ser adotadas para o algoritmo adaptativo. A primeira delas apresenta, se aplicada adequadamente, a vantagem de acelerar o processo de solução do problema não-linear, eliminando-se a necessidade de se repetir toda a análise sempre que o refinamento $p$ for realizado. Aplicar adequadamente a técnica adaptativa consiste em se controlar o erro a cada passo, mantendo-o suficientemente pequeno. Violando-se tal regra, arrisca-se não convergir para uma so-

\footnotetext{
${ }^{6}$ Caso se tenha interesse nesse tipo de estimador sugere-se a leitura dos trabalhos de GALLIMARD; LADEVÈZE; PELLE (1996) e GALLIMARD; LADEVĖZE; PELLE (2000), em que o erro estimado é integrado também ao longo de cada passo do processo de solução incremental.
} 
lução equilibrada ao final do passo em que a aproximação é enriquecida. Acrescenta-se a esse fato, as hipóteses adotadas na seção 6.6.1 para que o PVC (6.72) seja substituído pelo (6.73) e assim a medida $\left\|{ }^{t+\Delta t} \tilde{\boldsymbol{e}}_{p}\right\|$ forneça boas estimativas de erro.

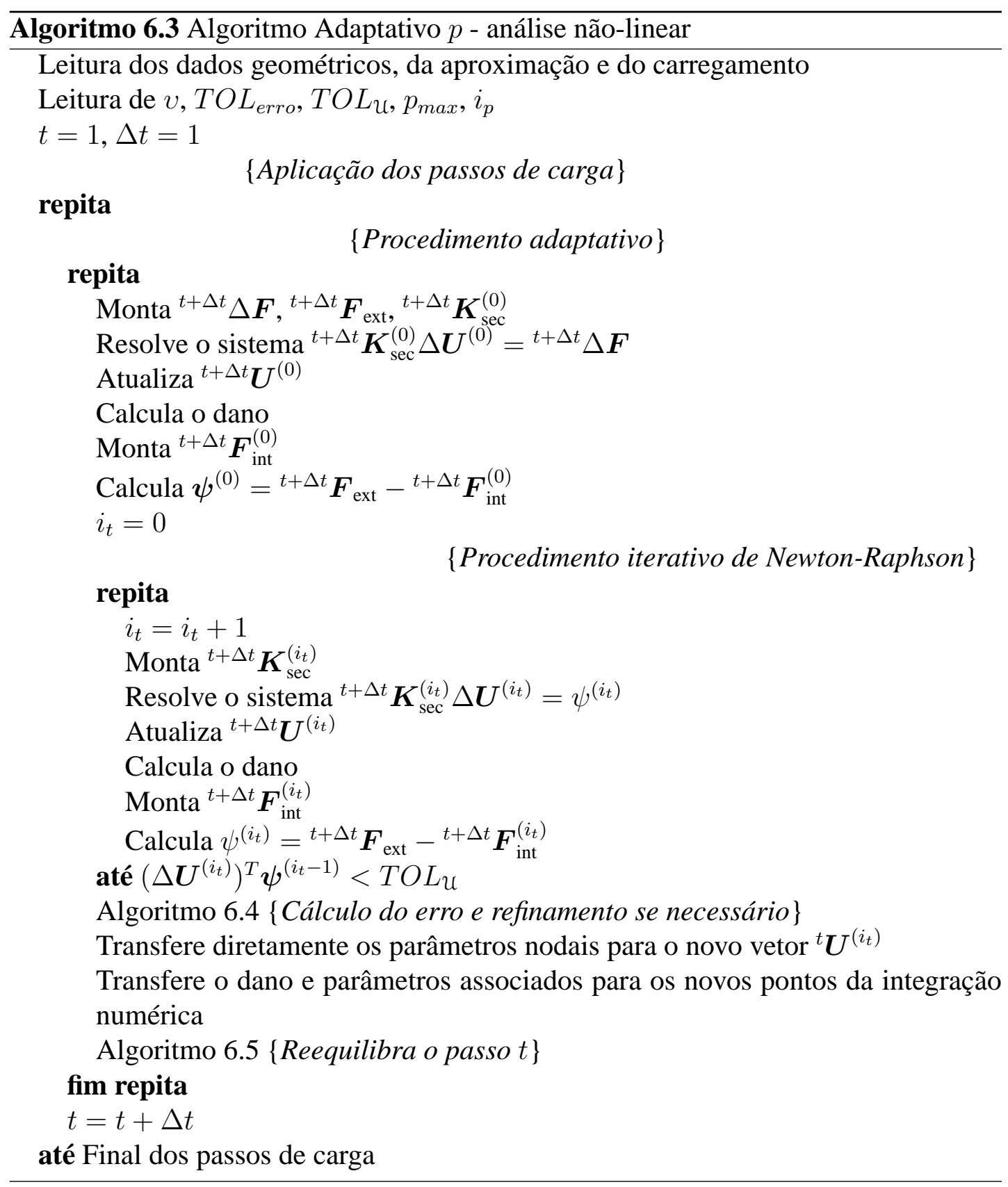

Com base nessa discussão, utiliza-se para as análises deste trabalho o algoritmo 6.3 em que apenas o passo de carga, para o qual a solução é refinada, é repetido. A medida $\left\|{ }^{t+\Delta t} \tilde{\boldsymbol{e}}_{p}\right\|_{u}$ é, portanto, calculada após ser verificado o equilíbrio ao final de cada passo $t+\Delta t$. Para que um novo incremento do carregamento seja aplicado é necessário que o erro relativo estimado seja inferior à tolerância $T O L_{\text {erro }}$ Caso contrário, 


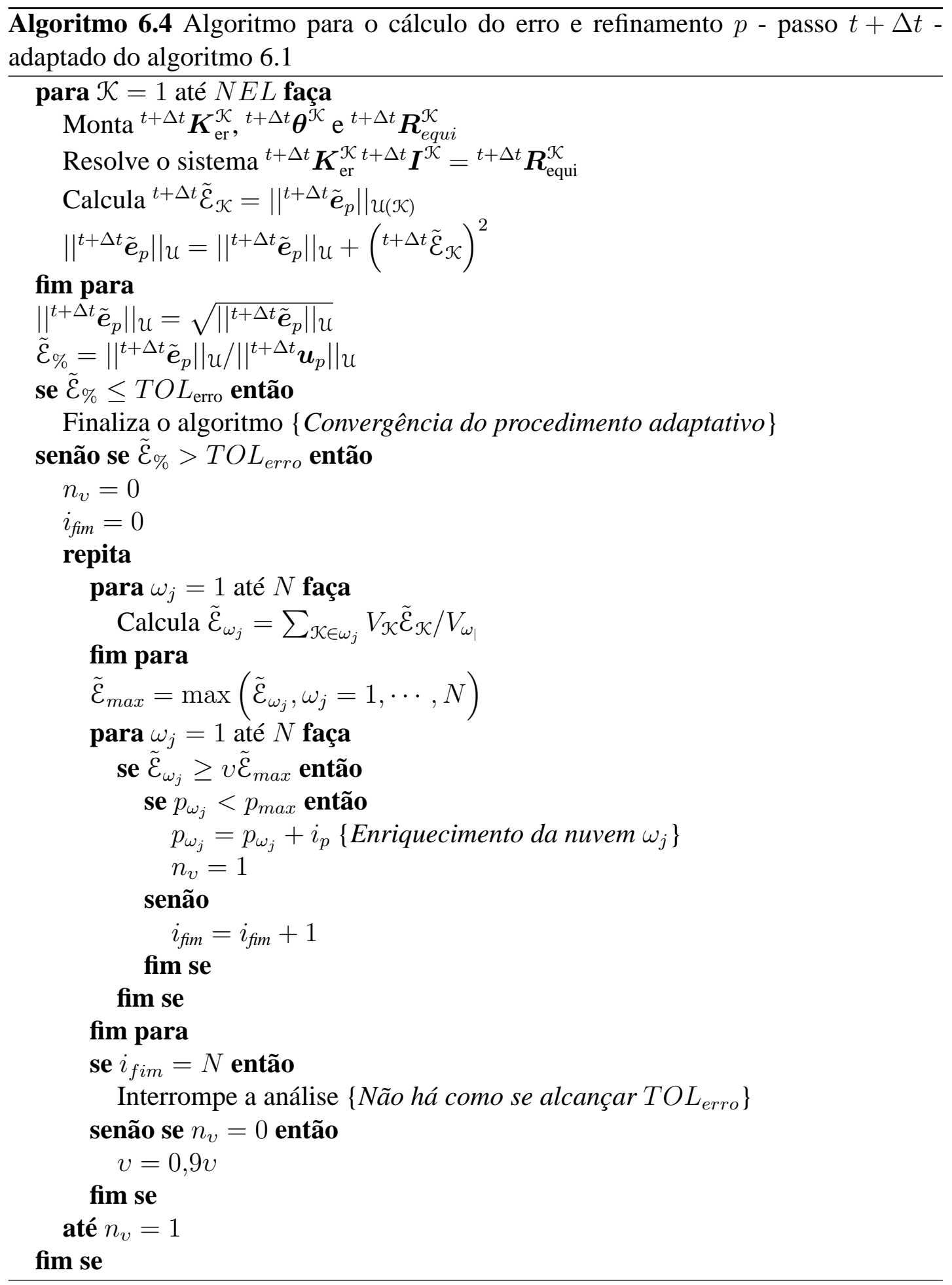


o enriquecimento das funções de PU é conduzido segundo os indicadores de erro por nuvens. Definida, então, uma nova aproximação para o problema, deve-se reaplicar o incremento de força do intervalo $\Delta t$. Antes, porém, as informações obtidas até o passo anterior, $t$, devem ser descritas segundo a nova aproximação. Tais informações correspondem às variáveis associadas aos nós e pontos de integração numérica que devem ser transferidas de acordo os novos graus de liberdade e nova ordem de quadratura definidos para a análise. As estratégias utilizadas para se realizar essa transferência são discutidas na seção 6.6.3 e devem ser tais que o novo conjunto de variáveis não viole as relações constitutivas do meio e o equilíbrio (no sentido generalizado da formulação fraca) ao final do passo $t$. Uma maneira de se contemplar tal objetivo consiste em se reequilibrar o passo $t$ com relação à nova descrição para as informações do problema exigida pelo refinamento adaptativo.

Em resumo, a seqüência refinamento, transferência de informações, reequilíbrio do passo anterior e equilíbrio do atual passo é repetida até que se alcance a tolerância $T O L_{\text {erro. }}$ Tal seqüência encontra-se representada no algoritmo 6.3. Por sua vez, o algoritmo 6.4 descreve o cálculo do erro e de seus indicadores conforme discutido na seção 6.6.1. Tal algoritmo corresponde a uma adaptação do algorimo 6.1. A escolha da versão em nuvens se deve unicamente por ser a mais próxima da abordagem do MEFG. O reequilíbrio do passo $t$ é esquematizado no algoritmo 6.5 da seção 6.6.3.

\subsubsection{Considerações sobre a Transferência das Variáveis}

Em um problema não-linear, em que o carregamento é aplicado de forma incremental, é necessário que as informações de um passo $t$ sejam transferidas para o passo $t+\Delta t$, uma vez que a solução é dependente da história do carregamento. Esta transferência é normalmente direta, desde que a descrição das variáveis representativas do problema não seja alterada. Com a introdução de um procedimento adaptativo, o refinamento é conduzido toda vez que, em um passo $t+\Delta t$, algum erro seja encontrado; como conseqüência, a discretização do problema é alterada dentro desse passo. A transferência das variáveis entre os passos $t$ e $t+\Delta t$ deixa, então, de ser direta o que torna necessário que uma estratégia consistente seja definida para esse fim. Várias são as propostas encontradas na literatura e, entre elas, podem ser citados os trabalhos de ORTIZ; QUIGLEY, IV (1991), RANNACHER; SUTTMEIER (1999) e BOROOMAND; ZIENKIEWICZ (1999).

Para o caso do MEFG dois grupos de variáveis devem ser transferidas. O primeiro 
corresponde aos parâmetros nodais, (3.16), usados para construir a função aproximada para os acréscimos de deslocamento no intervalo $\Delta t, \Delta \boldsymbol{u}_{p}$, e também a função aproximada dos deslocamentos até o final do passo $t,{ }^{t} \boldsymbol{u}_{p}$. Com o refinamento polinomial, novas funções são utilizadas para se descrever $\Delta \boldsymbol{u}_{p}$. Para se obter ${ }^{t+\Delta t} \boldsymbol{u}_{p}={ }^{t} \boldsymbol{u}_{p}+\Delta \boldsymbol{u}_{p}$ é necessário, portanto, que a função ${ }^{t} \boldsymbol{u}_{p}$ seja representada pelos mesmos parâmetros e funções de forma de ${ }^{t} \Delta \boldsymbol{u}_{p}$. O segundo grupo de variáveis está associado aos pontos da quadratura para a integração numérica. No caso de se empregar o modelo constitutivo de Mazars, seção 4.2, tais variáveis são $D, \varepsilon_{d}$ e, se a formulação tangente for adotada em (6.80), acrescentam-se as variáveis associadas à taxa de variação do dano com o tempo, apêndice A. Com o refinamento, a ordem polinomial das funções de aproximação é elevada e, por isso, um maior número de pontos de integração passa a ser necessário. Por essa razão, os valores calculados no passo $t$ para os pontos de integração devem ser transferidos para os novos pontos exigidos para a aproximação no intervalo $\Delta t$.

Assim como no MEF hierárquico, a estratégia de enriquecimento da aproximação no MEFG introduz novos graus de liberdade ao sistema, mantendo aqueles já existentes antes do refinamento polinomial. Por essa razão, não há necessidade de se utilizar nenhuma estratégia especial para a transferência dos parâmetros nodais, pois cada nova aproximação contém a anterior. Para melhor entender tal afirmação, considera-se a seguinte situação de refinamento nodal. Seja a nuvem $\omega_{j}$, sobre a qual encontram-se definidas as seguintes funções de aproximação, até a ordem polinomial $p$, e os correspondentes parâmetros nodais:

$$
\begin{gathered}
\left(\phi_{p}\right)_{j}^{T}=\left[\begin{array}{cccccccc}
\mathcal{N}_{j} & 0 & p_{j 1} \mathcal{N}_{j} & 0 & \cdots & 0 & p_{j q_{j}(p)} \mathcal{N}_{j} & 0 \\
0 & \mathcal{N}_{j} & 0 & p_{j 1} \mathcal{N}_{j} & 0 & \cdots & 0 & p_{j q_{j}(p)} \mathcal{N}_{j}
\end{array}\right] \\
\boldsymbol{U}^{T}=\left[\begin{array}{lllllll}
u_{j}^{x} & u_{j}^{y} & b_{j 1}^{x} & b_{j 1}^{y} & \cdots & b_{j q_{j}}^{x} & b_{j q_{j}}^{y}
\end{array}\right]
\end{gathered}
$$

Tenha-se, agora, em conta que o indicador de erro associado a esta nuvem exija seu enriquecimento para a ordem $p+1$. Sendo assim, um novo vetor para as funções de forma é definido da seguinte maneira: 


$$
\begin{aligned}
& { }_{\text {nova }}\left(\phi_{p}\right)_{j}^{T}=\underbrace{\left[\begin{array}{ccccccccc}
\mathcal{N}_{j} & 0 & p_{j 1} \mathcal{N}_{j} & 0 & \cdots & 0 & p_{j q_{j}(p)} \mathcal{N}_{j} & 0 \\
0 & \mathcal{N}_{j} & 0 & p_{j 1} \mathcal{N}_{j} & 0 & \cdots & 0 & p_{j q_{j}(p)} \mathcal{N}_{j}
\end{array}\right.}_{\left(\boldsymbol{\phi}_{p}\right)_{j}^{T}} \\
& \underbrace{\left.\begin{array}{cccccc}
p_{j q_{j}(p)+1} \mathcal{N}_{j} & 0 & \cdots & 0 & p_{j q_{j}(p+1)} \mathcal{N}_{j} & 0 \\
0 & p_{j q_{j}(p)+1} \mathcal{N}_{j} & 0 & \cdots & 0 & p_{j q_{j}(p+1)} \mathcal{N}_{j}
\end{array}\right]}
\end{aligned}
$$

Observa-se que não houve alteração nas funções anteriormente existentes, mas apenas um acréscimo de novos termos. Por esta razão, o novo vetor de parâmetros nodais é construído transferindo-se, diretamente, os valores pré-existentes em (6.86) e considerando-se como nulos os novos termos acrescentados:

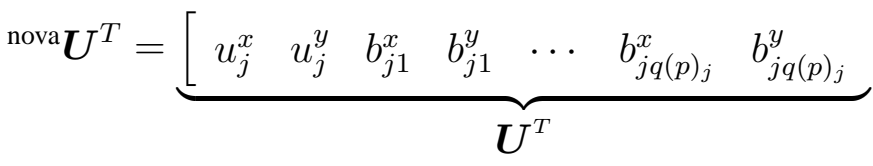

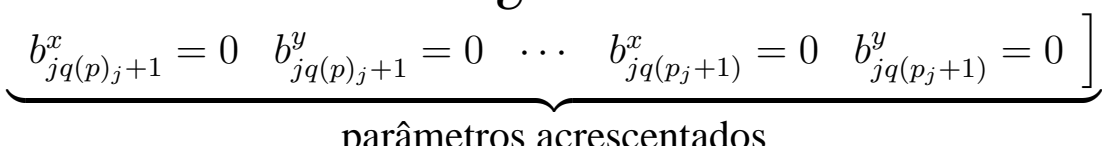

É esse o vetor utilizado para se calcular o vetor de forças internas no processo de reequilíbrio do algoritmo 6.5 .

Com relação às variáveis associadas aos pontos de integração, pode-se usar, por exemplo, a estratégia de transferência descrita no apêndice I. Tal estratégia é coerente com a abordagem do MEFG, pois utiliza o conceito da PU para "costurar" as expressões locais obtidas em cada nuvem para a variável a ser transferida.

Realizada a transferência das informações entre duas discretizações, é necessário verificar se a nova aproximação para o passo $t$ mantém-se consistente com o carregamento correspondente. Para isso devem ser respeitadas as seguintes condições:

- equilíbrio no sentido generalizado da formulação fraca do variacional;

- relações constitutivas entre os campos de deformação e tensão.

A estratégia de transferência descrita no apêndice I envolve apenas um conjunto de operações de projeção de valores discretos em funções regulares locais e sua interpolação para se ter uma representação global. Nenhuma restrição que garanta a 


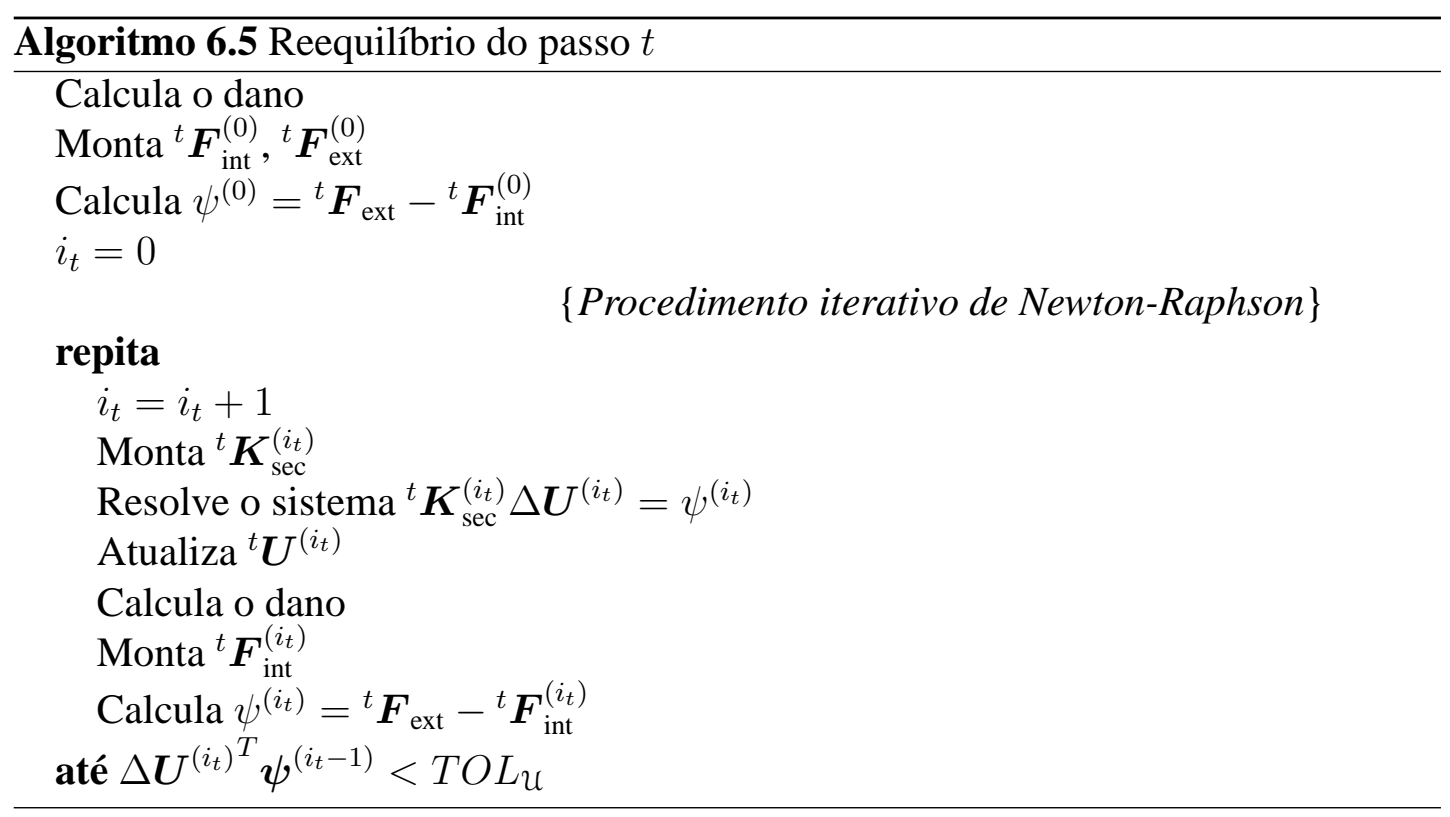

verificação destas condições é, portanto, introduzida. Esta tarefa pode ser realizada após a transferência das variáveis através do reequilíbrio do passo $t$ para a nova discretização. Dessa forma, o processo iterativo de solução de Newton-Raphson deve ser, novamente, aplicado sobre esse passo, até que novo equilíbrio seja assegurado. O procedimento é análogo ao utilizado para equilibrar as forças incrementais, sendo representado através do algoritmo 6.5. Corrige-se, assim, o campo de deslocamentos, segundo a nova discretização e ordem da quadratura, não apenas equilibrando as forças internas e externas como também garantindo a consistência entre os campos de tensão e deformação, BOROOMAND; ZIENKIEWICZ (1999).

\subsection{Exemplo Numérico}

Dois exemplos foram selecionados para ilustrar o emprego do MRE em análise não-linear de problemas estruturais resolvidos pelo MEFG.

O primeiro deles corresponde a uma chapa submetida à uma solicitação de compressão proveniente de uma força de volume. Este exemplo, e particularmente a solicitação por compressão, foram convenientemente escolhidos, em primeiro lugar porque o comportamento da estrutura danificada por compressão é bem mais estável do que sob tração. Além disso, até um certo limite de dano, a ser apresentado na próxima seção, a relação tensão-deformação nos pontos de Gauss ainda se encontra na fase de endurecimento e, por isso, a matriz de rigidez (6.82) é definida positiva; nessas condições ela pode ser utilizada diretamente na (6.80) para o cálculo das funções in- 
dicadoras de erro. Por meio deste exemplo procura-se mostrar que, respeitando-se às diversas considerações estabelecidas na seção 6.6.1, a medida de erro dada por (6.78) pode ser utilizada como uma estimativa para o erro, segundo a norma energia, ao final de cada passo do processo de solução do problema não-linear. Nesse sentido, os resultados numéricos são comparados aos obtidos em uma análise utilizando-se uma malha com elevado grau de refinamento $h$, cuja solução é tomada como a exata.

Como segundo exemplo, é apresentado o problema, mais complexo, de uma chapa com entalhe na qual o dano é produzido, principalmente por tensões de tração que se concentram em uma pequena região do meio. Este problema é, então, resolvido adaptativamente e a resposta global da estrutura é comparada a resultados experimentais. Apesar de violar algumas das condições apresentadas na seção 6.6.1 e que garantem uma boa estimativa global do erro, mostra-se que os indicadores ainda são capazes de identificar regiões com maior erro de aproximação, permitindo seu enriquecimento e a convergência para a solução procurada.

Duas observações devem ser feitas com relação aos procedimentos adotados para a estimativa do erro e análise adaptativa dos problemas aqui apresentados. Primeiramente, a técnica de transferência das variáveis, descrita no apêndice I, não foi utilizada. Em seu lugar, optou-se por manter constante o número de pontos da integração numérica desde o início da análise não-linear. Dessa forma, as posições em que as variáveis relacionadas com o dano são calculadas permaneceram as mesmas ao longo de todo o processo da solução incremental. O preço pago por esse procedimento é se ter, desde o primeiro passo de carga, um número elevado de pontos da quadratura numérica determinado pelo provável grau polinomial que a aproximação deve reproduzir ao final do carregamento. O ideal seria realizar a transferência das variáveis e permitir que, ao longo da análise, o número de pontos aumentasse conforme necessário.

No que se refere à integração numérica foi adotada a quadratura de Gauss-Lobatto. A razão dessa escolha está no cálculo dos vetores de forças residuais nodais ${ }^{t+\Delta t} \boldsymbol{R}_{\text {equi }}^{\mathcal{K}}$, (6.83). Uma vez que integração das tensões envolve pontos não apenas no interior do elemento, como também em seu contorno, torna-se necessário que se conheça o dano ao longo das faces de cada elemento. Uma primeira opção consistiria em se determinar o dano a partir das mesmas funções suavizadas utilizadas na estratégia de transferência de variáveis. Como tal procedimento não foi adotado, procurou-se resolver o problema de outra forma. A opção foi o emprego da quadratura de Gauss-Lobatto, que implica em se ter alguns dos pontos de integração localizados nas faces dos elementos. Dessa 
maneira, o dano calculado na fase de análise, para o interior e contorno dos elementos, pode ser diretamente utilizado na construção do vetor ${ }^{t+\Delta t} \boldsymbol{R}_{\text {equi }}^{\mathcal{K}}$;

\subsubsection{Chapa de Concreto Comprimida}

Na Figura 6.12 está representada uma chapa de concreto, com espessura $l_{z}=$ $10 \mathrm{~cm}$, solicitada à compressão por uma força de volume $b$. As condições de contorno naturais são representadas pela tensão de reação $q$ ao longo da face $A B$ que impede seu deslocamento na direção $x$. A estrutura encontra-se, portanto, equilibrada e os vínculos utilizados como condições de contorno essenciais tem a função de eliminar os movimentos de corpo rígido.

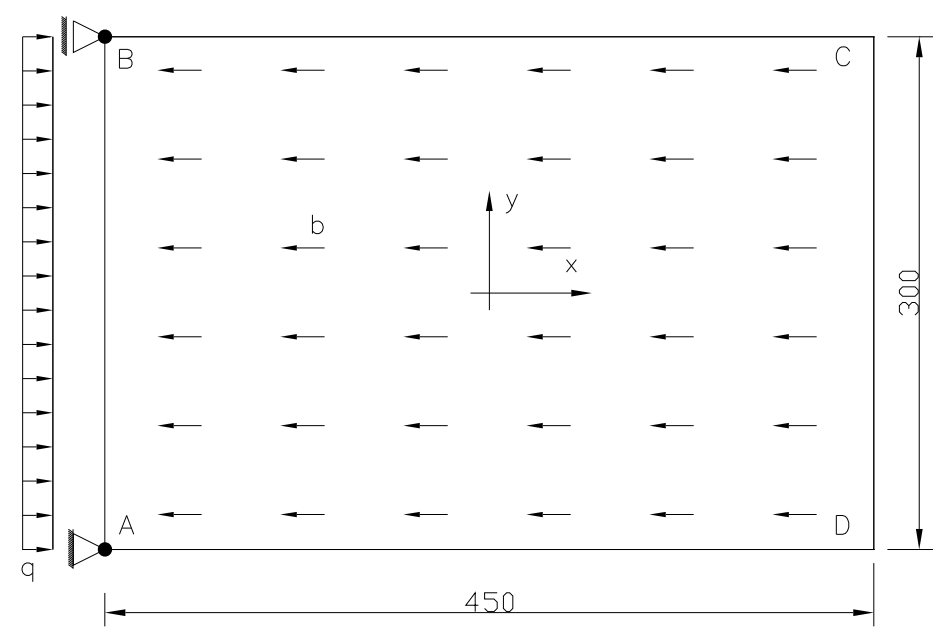

Figura 6.12: Chapa de concreto - geometria e condições de contorno - medidas em mm

Para simular o comportamento do meio é utilizado o Modelo de Mazars com abordagem local. Os seguintes parâmetros do modelo foram adotados:

$$
\begin{array}{cccc}
A_{T}=0,7 & B_{T}=8000 & A_{C}=0,85 & B_{C}=1050 \\
\varepsilon_{d 0}=0,000067 & E=29200 \mathrm{MPa} & \nu=0,2 & r_{\mathrm{nl}}=0
\end{array}
$$

A curva de tensão versus deformação, para o caso de solicitação uni-axial, exibida na Figura 6.13, é ascendente até o valor de $\varepsilon \approx-0,003$. A partir deste ponto a tangente à curva torna-se negativa. Para o cálculo das funções indicadoras de erro em um elemento $\mathcal{K}$ é necessário montar a matriz ${ }^{t+\Delta t} \boldsymbol{K}_{\mathrm{er}}^{\mathcal{K}}$, expressão (6.82), a partir da contribuição de parcelas relativas a cada ponto de Gauss desse elemento. Para se garantir que ${ }^{t+\Delta t} \boldsymbol{K}_{\text {er }}^{\mathcal{K}}$ seja sempre definida positiva, é necessário que as deformações no elemento $\mathcal{K}$ sejam inferiores a $-0,003$, correspondendo a um dano próximo de 0,6 . 
Tal condição é assegurada definindo-se a força de volume $b=1000 \mathrm{kN} / \mathrm{m}^{3}$ e a tensão de superfície $q=450 \mathrm{kN} / \mathrm{m}^{2}$.

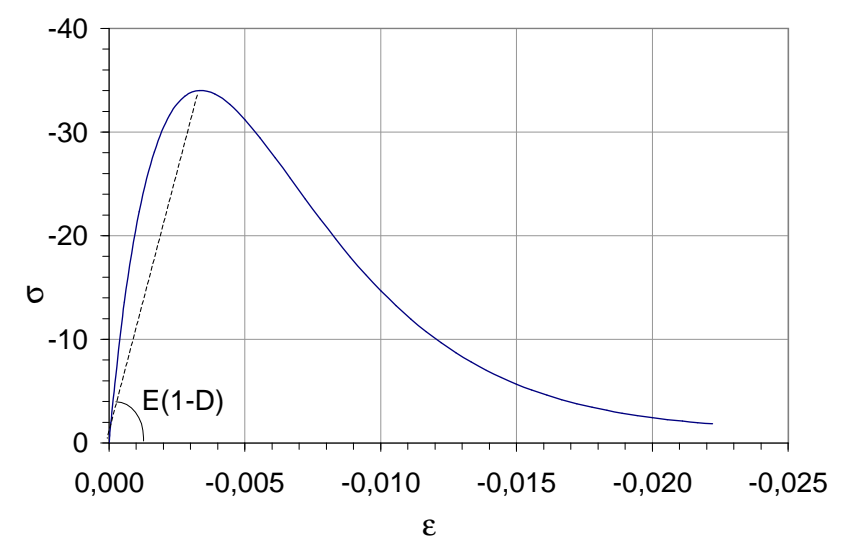

Figura 6.13: Relação uni-axial tensão $\times$ deformação

Duas malhas com aproximação linear foram utilizadas para a discretização do problema e encontram-se representadas na Figura 6.14. A solução obtida com a malha da Figura 6.14(a), ao final de cada passo $t+\Delta t$, tem seu erro estimado pelo MRE, empregando-se a norma energia $\left\|^{t+\Delta t} \tilde{\boldsymbol{e}}_{p}\right\|_{u}$. Para a representação das funções indicadoras de erro em cada elemento $\mathcal{K}$ é empregado o seguinte espaço de funções bolha:

$$
X_{2}^{0}(\mathcal{K})=\left\{\boldsymbol{v}_{2}^{0} \in X_{2}(\mathcal{K}) \subset \mathcal{H}^{1} ; \Pi_{p}\left(\boldsymbol{v}_{2}^{0}\right)=0, \boldsymbol{v}_{2}^{0}=0 \text { em } \Gamma_{D}\right\}
$$

gerado pelas funções de forma:

$$
\left(\boldsymbol{\Phi}_{2}^{0}\right)_{j}^{T}=\left[\begin{array}{cccc}
\mathcal{N}_{j}\left(\frac{x-x_{j}}{h_{j}}\right) & 0 & \mathcal{N}_{j}\left(\frac{y-y_{j}}{h_{j}}\right) & 0 \\
0 & \mathcal{N}_{j}\left(\frac{x-x_{j}}{h_{j}}\right) & 0 & \mathcal{N}_{j}\left(\frac{y-y_{j}}{h_{j}}\right)
\end{array}\right]
$$

sendo $h_{j}$ neste caso corresponde à diagonal dos elementos usados para a análise.

A medida $\left\|^{t+\Delta t} \tilde{\boldsymbol{e}}_{p}\right\|_{u}$ é comparada ao resultado do seguinte produto interno:

$$
{ }^{t+\Delta t}\left(\boldsymbol{\sigma}_{\mathrm{er}}, \boldsymbol{\varepsilon}_{\mathrm{er}}\right)=\sqrt{\left.\iint_{\Omega}\left[\boldsymbol{\sigma}\left({ }^{t+\Delta t} \boldsymbol{u}\right)-\boldsymbol{\sigma}\left({ }^{t+\Delta t} \boldsymbol{u}_{p}\right)\right]\left[\boldsymbol{\varepsilon}^{(t+\Delta t} \boldsymbol{u}-{ }^{t+\Delta t} \boldsymbol{u}\right)\right] l_{z} d x d y}
$$

A razão entre esses dois valores determina o índice de efetividade em cada passo de carga. Nota-se que, sendo um problema não-linear, não há como definir a norma ener- 
gia do erro como na expressão (6.8).

$\mathrm{Na}$ ausência da solução analítica exata, adotou-se em seu lugar, a solução numérica obtida com a malha da Figura 6.14(b), definindo-se a partir dela as correspondentes tensões e deformações presentes ao final de cada passo na expressão (6.90).

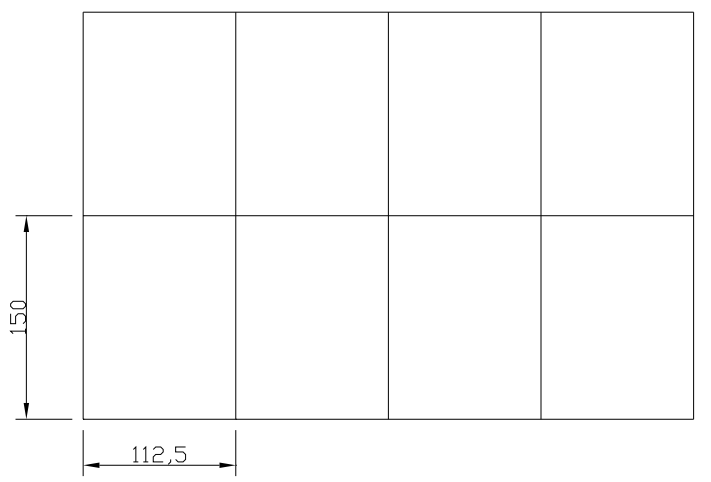

(a) 8 elementos, aproximação linear

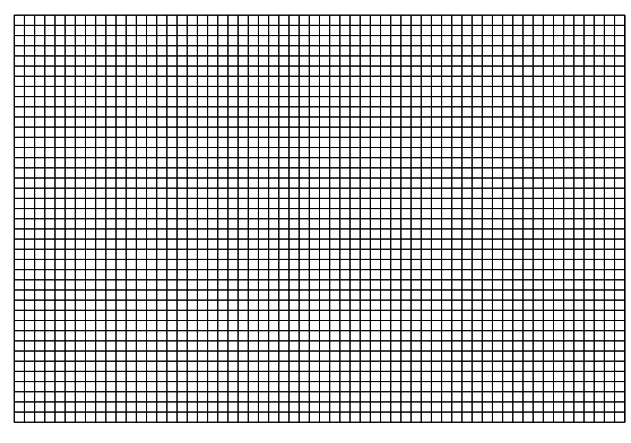

(b) 2400 elementos, aproximação linear

Figura 6.14: Malhas utilizadas - medidas em mm

Para a análise em estado plano de tensão e aplicação do MRE foram feitas as seguintes considerações:

montagem da matriz de rigidez: formulação secante para a matriz utilizada em (5.9) e formulação tangente, de acordo com as expressões (6.82) e (6.84) para o cálculo das funções de erro;

solução do sistema de equações: o método de solução escolhido para as análises com as duas malhas foi o dos Gradientes Conjugados. Essa opção por um método iterativo é justificada pela ordem relativamente alta do sistema de equações obtido na análise com a malha de 2400 elementos. Por sua vez, no cálculo das funções de erro locais, problema (6.80), com a malha de 8 elementos, utilizou-se o procedimento de Babuška com tolerância de $1 \times 10^{-10}$ e perturbação da diagonal principal $\epsilon=1 \times 10^{-8}$;

integração numérica: na malha de 2400 elementos foram usados $2 \times 2$ pontos de Gauss-Legendre. Já na malha de 8 elementos, para melhor representar a distribuição do dano foram empregados $12 \times 12$ pontos de Gauss-Lobatto;

equilíbrio: a convergência do processo iterativo de solução do Método de NewtonRaphson foi averiguada pelo critério em energia, adotando-se uma tolerância de 


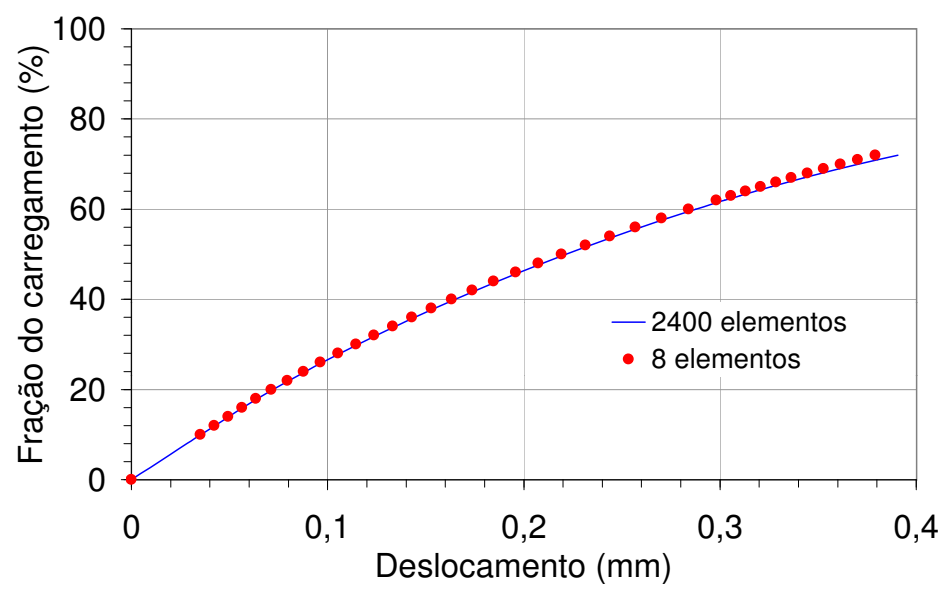

Figura 6.15: Resposta global da estrutura

0,001\% com relação ao primeiro passo elástico. A força de volume $b$ e a tensão de superfície $q$ foram introduzidas em 65 incrementos: um primeiro passo com $10 \%$ do total do carregamento, seguido de 26 passos com $2 \%$ e outros 38 com $1 \%$. No $42^{\circ}$ passo, a análise com a malha de 2400 elementos foi interrompida devido à divergência das soluções sucessivamente obtidas com o Método de Newton-Raphson. Por essa razão, para as duas análises, apenas os resultados até o passo 41 , equivalente a $76 \%$ do carregamento total, são exibidos no gráfico da Figura 6.15. No eixo das abscissas são representados os deslocamentos horizontais medidos no ponto médio da face $C D$ da Figura 6.12.

A título de ilustração, na Figura 6.16 são representadas as distribuições de dano, ao final do passo 41, para as análises realizadas com as malhas de 8 e 2400 elementos. A diferença observada reflete a pequena divergência entre as respostas globais para as duas análises registrada na Figura 6.15.

O erro relativo estimado para a aproximação da malha com 8 elementos foi de $12,84 \%$ para o primeiro passo elástico, chegando a $16,55 \%$ ao final do passo 41 . A medida $\left\|{ }^{t+\Delta t} \tilde{\boldsymbol{e}}_{p}\right\|_{u}$ foi avaliada por meio do índice global de efetividade $\theta$, calculado em cada passo e representado no gráfico da Figura 6.17. De acordo com os resultados obtidos, conclui-se que, para este problema, os valores de $\left\|{ }^{t+\Delta t} \tilde{\boldsymbol{e}}_{p}\right\|_{u}$ formam um bom estimador para o erro, medido segundo o produto (6.90). Deve-se, entretanto, observar que determinadas características deste problema favoreceram a excelência dos resultados. O dano, como mostrado nos "mapas de dano" da Figura 6.16 está limitado a 0,6. Dessa forma, em todos os elementos ainda se encontra uma situação equivalente 


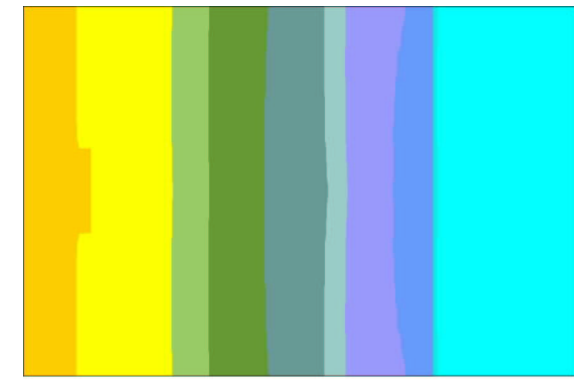

(a) 8 elementos, aproximação linear

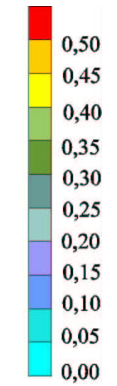

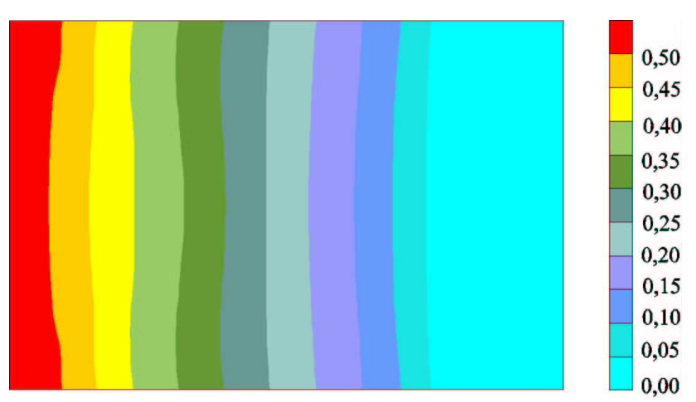

(b) 2400 elementos, aproximação linear

Figura 6.16: Mapa da distribuição do dano no passo 41

ao trecho de endurecimento da curva 6.13. Nenhuma restrição existe, portanto, ao emprego da expressão tangente (6.84) na construção das matrizes (6.82). Além disso, foram usados passos bem pequenos, especialmente a partir do instante em que se observa uma maior a evolução do dano. Sendo assim, garantiu-se, em cada passo, que o problema (6.73) pudesse ser usado no lugar de (6.72), como sugerido na seção 6.6.1.

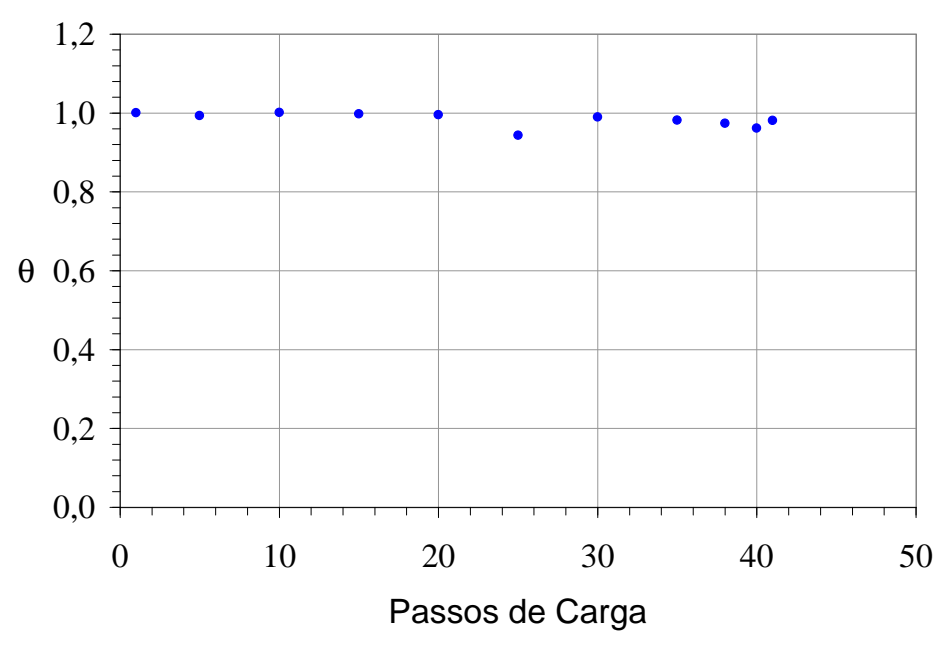

Figura 6.17: Índice de efetividade global em cada passo

\subsubsection{Chapa com Entalhe}

O problema representado na Figura 6.18 corresponde a uma chapa de concreto com $12 \mathrm{~cm}$ de espessura, solicitada por duas forças horizontais $F$ que provocam a abertura do entalhe. Em MAZARS (1984) esse caso foi utilizado para se confrontar o resultado de uma análise numérica empregando-se o modelo de Mazars com a resposta 
real da estrutura obtida experimentalmente.

A presença do entalhe de extremidade arredondada produz uma concentração das tensões em sua vizinhança sem, no entanto, permitir o aparecimento de singularidades. Este peculiar campo de tensões induz à concentração de micro-defeitos na região próxima ao entalhe, os quais evoluem na medida em que a estrutura é solicitada. Para o nível de carga máxima os micro-defeitos dão origem a uma trinca macroscópica cuja propagação instável determina o ruptura do meio.

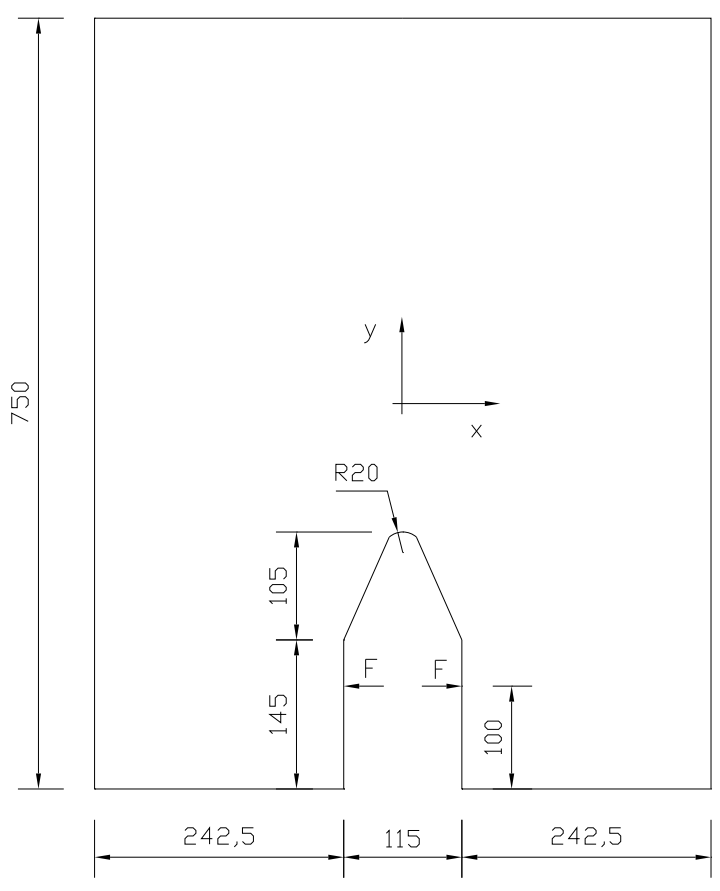

Figura 6.18: Chapa de concreto com entalhe - geometria e solicitação - medidas em mm

A análise numérica, apresentada a seguir, envolve apenas a fase de formação e evolução de micro-defeitos, simulada com o modelo de Mazars, segundo uma abordagem não-local e adotando-se os seguintes parâmetros:

$$
\begin{array}{cccc}
A_{T}=0,8 & B_{T}=20000 & A_{C}=1,4 & B_{C}=1850 \\
\varepsilon_{d 0}=0,000123 & E=30000 \mathrm{MPa} & \nu=0,2 & l_{c}=3 \mathrm{~cm}
\end{array}
$$

A malha empregada para se construir as funções da PU encontra-se representada na Figura 6.19, juntamente com as as condições de contorno adotadas. Nota-se que, da mesma maneira que nos demais exemplos em análise bi-dimensional, a força concentrada indicada na Figura 6.18 foi substituída por uma força distribuída equivalente $q$. Os vínculos nodais foram introduzidos com a finalidade de eliminar os movimentos de corpo rígido, uma vez que o carregamento é auto equilibrado. Para se determinar 
o enriquecimento polinomial em cada nuvem, o problema não-linear foi resolvido de acordo com o algoritmo adaptativo 6.3 e as funções indicadoras de erro foram descritas no espaço definido por (6.30).

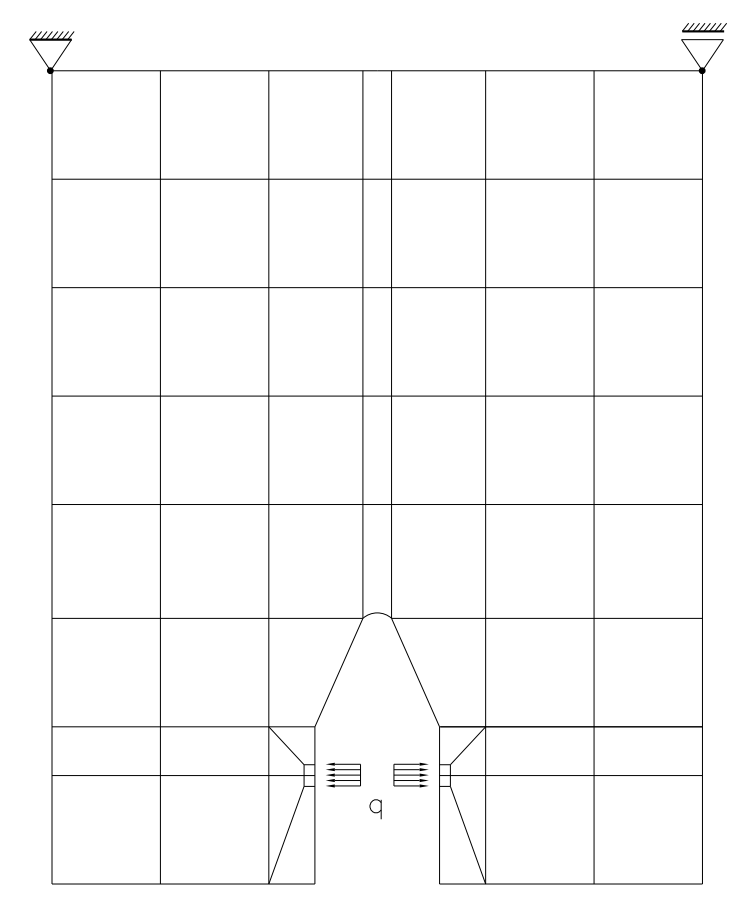

Figura 6.19: Malha e condições de contorno utilizadas para a análise

Para a análise numérica e estimativa de erro foram adotados:

aproximação do contorno curvo: foram utilizadas as funções de mistura (blending function);

análise não-local: como o comprimento característico do material $l_{c}$ vale $3 \mathrm{~cm}$, o raio adotado para a análise não-local foi de $1,5 \mathrm{~cm}$, conforme definido na seção 4.4;

estado plano de deformação: para este tipo de problema em que se espera uma elevada concentração do dano de tração em uma pequena região, torna-se interessante adotar o estado plano de deformação; dessa forma, perpendicularmente ao plano de representação da estrutura, aparecem tensões de compressão na região do entalhe, o que estabiliza uma possível evolução prematura do dano;

equilíbrio: aplicou-se o Método de Newton-Raphson para a solução do problema nãolinear físico. Como critério de convergência foi fixada uma tolerância de $1 \%$ na norma de energia incremental com relação ao primeiro passo elástico. A força 
aplicada de $18,5 \mathrm{kN}$ foi dividida em 10 passos, sendo os dois primeiros de $5 \mathrm{kN}$, seguidos de dois passos de $2 \mathrm{kN}$, três de $1 \mathrm{kN}$ e os três últimos de $0,5 \mathrm{kN}$;

montagem da matriz de rigidez: para a montagem da matriz de rigidez secante, empregada no problema (5.9), limitou-se o dano ao valor de 0,87. Já as matrizes por elemento, utilizadas no cálculo das funções indicadoras de erro, foram construídas sem limitação na variável dano. A expressão (6.82) foi, entretanto, modificada substituindo-se a relação constitutiva tangente (6.84) pela secante (4.9). A razão para esse procedimento é justificada mais adiante no item referente ao estimador de erro;

solução do sistema de equações: tanto na fase de análise, problema (5.9), quanto na estimativa de erro (6.80), foi utilizado o procedimento de Babuška, adotando-se $T O L=10^{-10}$ e $\epsilon=10^{-12}$;

estimador de erro: como já comentado anteriormente, o mecanismo de danificação neste problema é produzido exclusivamente pelas tensões de tração. No modelo de Mazars, o dano de tração implica imediatamente em perda de resistência local. Por essa razão, nos pontos danificados, a relação tensão-deformação é decrescente e a contribuição para a matriz ${ }^{t+\Delta t} \boldsymbol{K}_{\mathrm{er}}^{\mathcal{K}}$ definida em (6.82) deixa de ser definida positiva. Como conseqüência, viola-se a condição estabelecida em RHEINBOLDT (1985) e discutida na seção 6.6.1, para que a função ${ }^{t+\Delta t} \boldsymbol{e}_{p}^{*}$ seja assintoticamente equivalente ao erro exato da aproximação ${ }^{t+\Delta t} \boldsymbol{e}_{p}$. Além disso, o emprego da forma tangente na expressão (6.75), do problema variacional do erro (6.74), pode provocar o mal condicionamento da matriz ${ }^{t+\Delta t} \boldsymbol{K}_{\mathrm{er}}^{\mathcal{K}}$. Considerando-se esses fatos, optou-se por substituir a forma tangente ${ }^{t+\Delta t} \boldsymbol{K}_{\text {er }}^{\mathcal{K}}$ pela secante. A medida de erro global obtida não pode, portanto, ser considerada, verdadeiramente, como um estimador de erro, apesar de continuar sendo empregada no procedimento adaptativo para se definir a necessidade ou não do refinamento. As funções indicadoras de erro também deixam de ser estimadores locais, mas ainda assim permitem a condução do processo de refinamento como se observa pelos resultados apresentados ao final desta seção;

algoritmo adaptativo: o algoritmo 6.3 é empregado adotando-se $T O L_{\text {erro }}=15 \%$, $v=0,5$ e $p_{\max }=8$

restrições ao refinamento: a função que representa as condições de contorno natu- 
rais é descontínua e, por isso, alguma concentração de tensões é observada nos pontos em que a carga distribuída deixa de existir. Detectado pelo indicador de erro, esse fato pode conduzir a um refinamento desnecessário na região do carregamento. Por essa razão, nas nuvens próximas à região de aplicação da carga limitou-se a 2 o grau do enriquecimento polinomial;

integração numérica: foram utilizados, na maioria dos elementos, $4 \times 4$ pontos de Gauss-Lobatto, aumentando-se esse número proximamente aos elementos de face curva, onde foram empregados $15 \times 15$ pontos. Tal procedimento visou não só garantir a integração quando a aproximação passou a ser enriquecida com polinômios de elevado grau durante o refinamento adaptativo, como também captar o dano próximo da raiz do entalhe.

No gráfico da Figura 6.20 estão representadas as curvas das respostas experimentais, MAZARS (1984), e numérica referentes à abertura do entalhe, $\delta$, medida no ponto de aplicação da força $F$ (na verdade ponto médio da força distribuída equivalente).

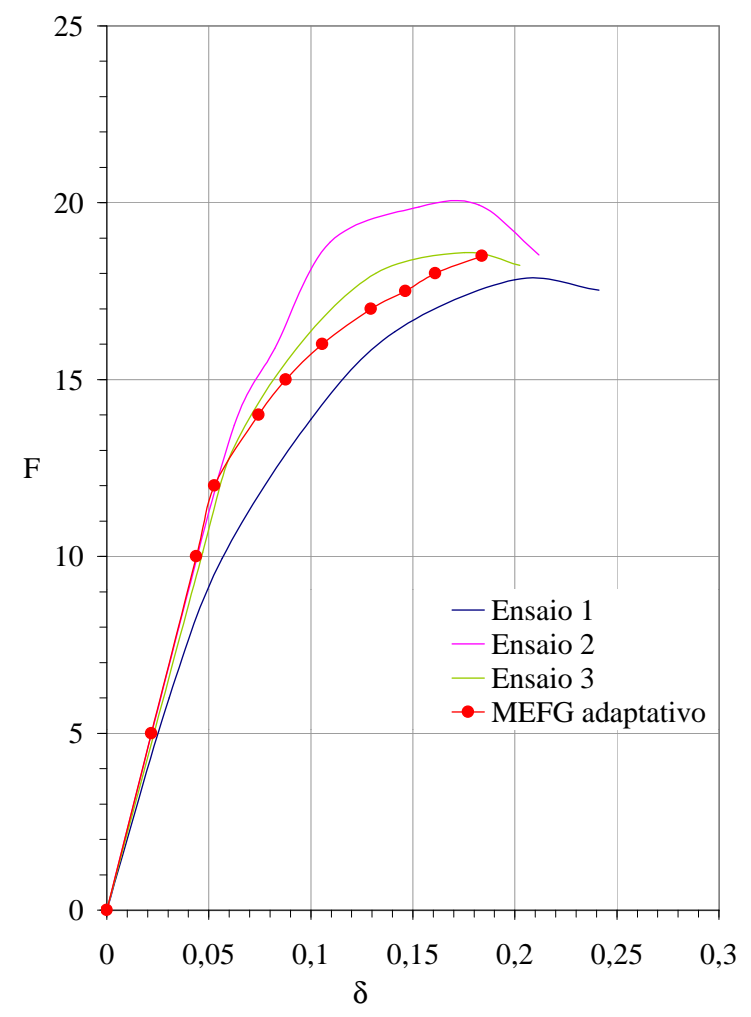

Figura 6.20: Resposta global da estrutura 
O processo adaptativo de solução foi caracterizado por dois momentos de refinamento polinomial. Ainda no primeiro passo elástico, chegou-se à discretização ilustrada pela Figura 6.21(a), após uma sequiência de 5 iterações do procedimento adaptativo. Tal discretização permaneceu inalterada até o passo 7, quando novo refinamento adaptativo foi realizado, dessa vez com 2 iterações, Figura 6.21(b).

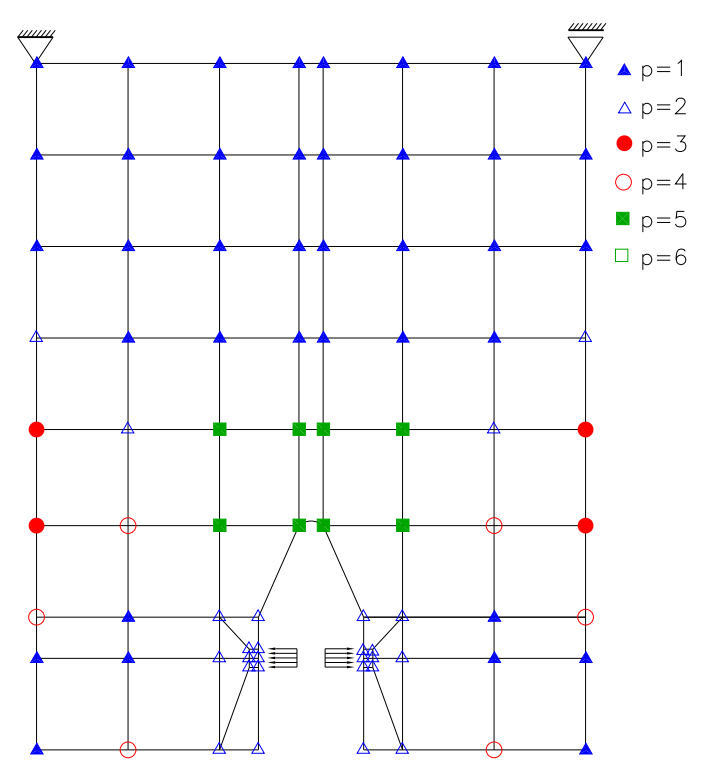

(a) Discretização utilizada até o passo 7

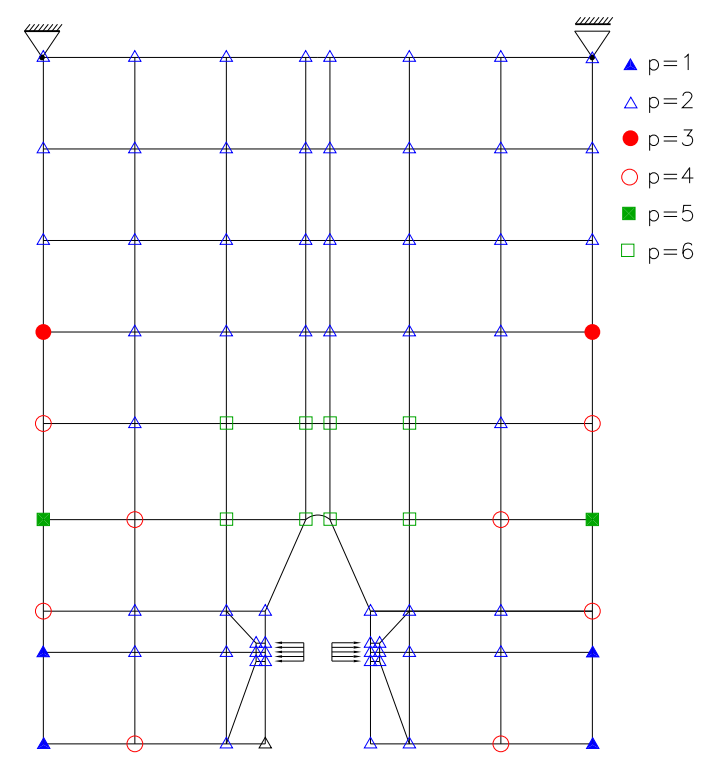

(b) Discretização utilizada após o passo 7

Figura 6.21: Discretizações definidas pelo algoritmo adaptativo

Devido às várias simplificações realizadas no cálculo da medida $\left\|\left.\right|^{t+\Delta t} \tilde{\boldsymbol{e}}_{p}\right\|_{u}$, não se pode garantir que o erro realmente esteja limitado a $15 \%$ ao final de cada passo $\Delta t$. Pode-se, contudo, averiguar, pelo resultado obtido, que os indicadores de erro possibilitaram a determinação de uma boa aproximação para a resposta real da estrutura. É possível, assim, partindo-se de uma malha relativamente simples de elementos, em sua maioria, regulares, simular adequadamente o comportamento não-linear de uma estrutura de concreto caracterizado pelo fenômeno de danificação, Figura 6.22. Além disso, graças à estratégia de enriquecimento do MEFG não há necessidade de se impor restrições às aproximações para que estas sejam compatibilizadas entre elementos, o que simplifica bastante a implementação do processo adaptativo. 


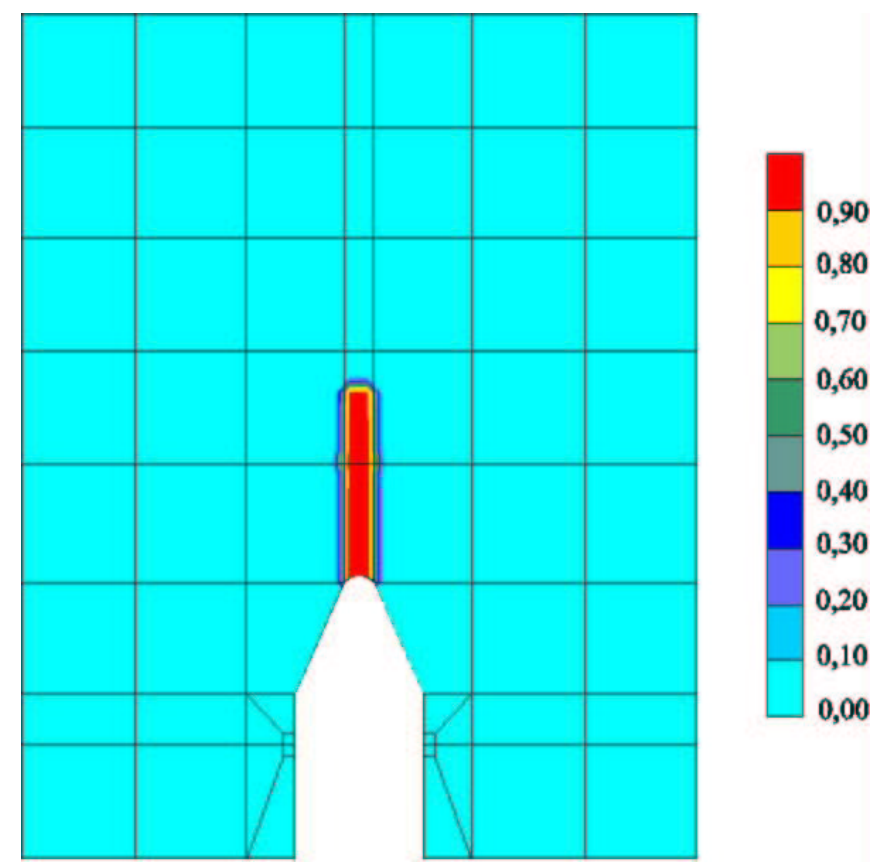

Figura 6.22: Distribuição do dano ao final do carregamento $(F=18,5 \mathrm{kN})$ 


\section{Capítulo 7}

\section{Considerações Finais}

Neste capítulo de considerações finais, procura-se, essencialmente, fornecer uma visão geral do trabalho e de suas contribuições. As conclusões estabelecidas com base nos resultados e observações dos capítulos anteriores são, sobretudo, motivadoras de uma extensa pesquisa ainda a ser realizada e que compreende o desenvolvimento de uma eficiente ferramenta numérica para análise do comportamento de estruturas governado pela propagação de descontinuidades. Este trabalho é, portanto, princípio de

uma pesquisa e não sua finalização. É, por essa razão, que a última seção é dedicada às propostas para desenvolvimentos futuros.

\subsection{Síntese e Conclusões}

Ao longo deste texto foram apresentadas análises numéricas realizadas com algumas das formulações sem malha, o MGLE e o Método das Nuvens $h p$, e com o MEFG. $\mathrm{Na}$ descrição desses métodos procurou-se apresentá-los não de forma dissociada mas interligando-os segundo um novo conceito de discretização que culmina com a abordagem do MEFG. Seja como uma particularização do Método das Nuvens $h p$, seja como uma generalização do MEF, o MEFG pode ser entendido como uma "ponte" entre estas duas abordagens, reunindo as formulações com e sem elementos sob um mesmo enfoque. Não há, portanto, neste trabalho, razão para postular a superioridade de qualquer um desses métodos em relação aos outros, mas a idéia é colocar em destaque suas vantagens, conforme a aplicação que se queira dar a cada um deles.

O trabalho foi inicialmente exploratório e, por isso, diversos resultados da literatura foram reproduzidos. Tais resultados serviram para esmiuçar detalhes de implementação, salientando-se vantagens e limitações dos métodos numéricos utilizados. 
A aplicação selecionada para se avaliar o desempenho destas novas abordagens corresponde à análise de estruturas em regime de comportamento não-linear físico. Dois fatores justificaram a opção pela Mecânica do Dano Contínuo como ferramenta para a idealização do comportamento do material. Primeiramente, não havia na literatura referências sobre análises com dano conduzidas com os métodos estudados. Além desse fato, existia a expectativa de que a liberdade no refinamento da solução por meio da técnica de enriquecimento, especialmente do MEFG, permitiria simular com melhor qualidade e eficiência a propagação dos micro-defeitos no meio. De fato, os resultados de exemplos numéricos reunidos nos capítulos 5 e 6 evidenciaram a potencialidade do método. Por exemplo, partindo-se de malhas com poucos elementos conseguiu-se reproduzir adequadamente as respostas globais de estruturas, obtidas experimentalmente.

A independência da malha, apesar de relativa, como comentado na seção 3.2.1, confere ao MEFG uma grande flexibilidade, pois permite que o refinamento da solução seja realizado apenas nas regiões em que esse seja realmente necessário. Tal característica foi explorada, de maneira intuitiva, no problema da viga de concreto armado na seção 5.3.1. Os bons resultados, então encontrados, motivaram a introdução de medidas de erro local e global com vistas à realização de análises adaptativas. O MRE já bem fundamentado para o MEF, foi adotado como estratégia para o cálculo dessas medidas de erro. O espaço de aproximação do erro, a técnica de equilíbrio (para que a formulação dos problemas locais dos indicadores de erro seja consistente) e o algoritmo adaptativo normalmente adotado, tiveram que ser adaptados à abordagem do MEFG. A aplicação do estimador para problemas não-lineares passou por uma série de simplificações que, de certa forma, comprometeram algumas de suas propriedades, como a garantia de ser assintoticamente exato. Nos exemplos apresentados, seção 6.7, mostrou-se que, apesar disso, as medidas de erro local e global obtidas podem ser adequadamente utilizadas para a condução de um processo adaptativo em análise nãolinear.

Resumindo, com base nas observações e nos resultados registrados ao longo deste trabalho, comprovam-se as vantagens do MEFG, também para a simulação do comportamento não-linear de estruturas governado pelo dano. Em particular, o refinamento da solução, realizado pelo enriquecimento da PU, é de simples implementação e não exige, como no MEF, restrições para a compatibilização das aproximações entre elementos vizinhos. As funções de forma de caráter polinomial reproduziram bem o 
campo de deslocamentos para diversos níveis de dano. Foi essencial empregar uma abordagem não-local para o modelo constitutivo, o que possibilitou obter soluções relativas a diferentes distribuições de dano, dispersas pelo meio ou concentradas em regiões específicas. Funções não-polinomiais podem ser também utilizadas para melhor reproduzir a configuração das regiões danificadas, mas para isso um estudo minucioso deve ser empreendido. Outra conclusão importante é a de que o procedimento adaptativo implementado, ainda que baseado em medidas de erro simplificadas, tornou possível uma análise não-linear eficiente na medida que novos graus de liberdade foram acrescentados ao sistema apenas quando necessários.

O trabalho permitiu, assim, estabelecer os primeiros passos no sentido de se ter uma ferramenta numérica direcionada à aplicação de problemas não-lineares regidos pela propagação de defeitos. Ainda em termos da continuidade da pesquisa, outro campo bastante promissor para o emprego do MEFG encontra-se na simulação da transição entre o dano e o surgimento da trinca macroscópica, sendo esse o assunto da próxima seção.

\subsection{Dano e Fratura}

Dano e fratura são dois fenômenos interligados que se manifestam em diferentes escalas, sendo objeto de estudo através das Mecânicas do Dano Contínuo e da Fratura. Na primeira destas teorias, discutida no capítulo 4, o processo de danificação difusa no material real é levado em conta em um meio contínuo equivalente, mediante variação de suas mecânicas. Uma das limitações para a aplicação dos modelos constitutivos de dano está na hipótese de que os micro-defeitos devem estar dispersos pelo meio. Para determinados problemas, como a viga bi-apoiada discutida na seção 5.3.1, a disposição do carregamento e a presença da armadura colaboram para que, mesmo em escala macroscópica, as fissuras sejam distribuídas ao longo da região central, solicitada por momento fletor constante. Nessas condições, a simulação numérica com o modelo de Mazars ainda se mostra uma aproximação razoável para o problema físico analisado. Por outro lado, no problema da seção 6.7.2, a configuração do dano é bem diferente, caracaterizando-se pela sua concentração na região do entalhe. Com o emprego de um modelo de dano, a curva representada na Figura 6.20 somente foi obtida graças à abordagem não-local. É importante registrar que prosseguindo-se com a análise numérica para além de $18,5 \mathrm{kN}$, o resultado encontrado, torna-se divergente com relação a 
resposta real da estrutura observada experimentalmente em MAZARS (1984). Nesse nível de carregamento começa a se formar uma trinca macroscópica, cuja propagação deve governar o comportamento estrutural até o seu colapso. Claramente, o fenômeno incidente sobre o material passa, então, a pertencer ao domínio da Mecânica da Fratura.

O problema da chapa com entalhe é, portanto, um interessante exemplo cuja simulação completa exigiria um processo de transição entre as teorias de dano e fratura. Uma proposta nesse sentido é discutida em BAZǍNT; PIJAUDIER-CABOT (1989), e consiste em se ter um primeiro estágio da análise conduzido por algum modelo de dano não-local, para se determinar a zona em que o os micro-defeitos se localizam. A partir desses resultados, determina-se uma trinca equivalente relacionando-se a energia de fratura e a dissipada pela evolução do dano. Entretanto, diversas questões, entre elas como associar de forma consistente a energia de fratura com os parâmetros do modelo de dano, bem como a definição da lei que governa a propagação da trinca no meio danificado, precisam ser melhor compreendidos para que a transição entre esses dois fenômenos seja resolvida de forma satisfatória.

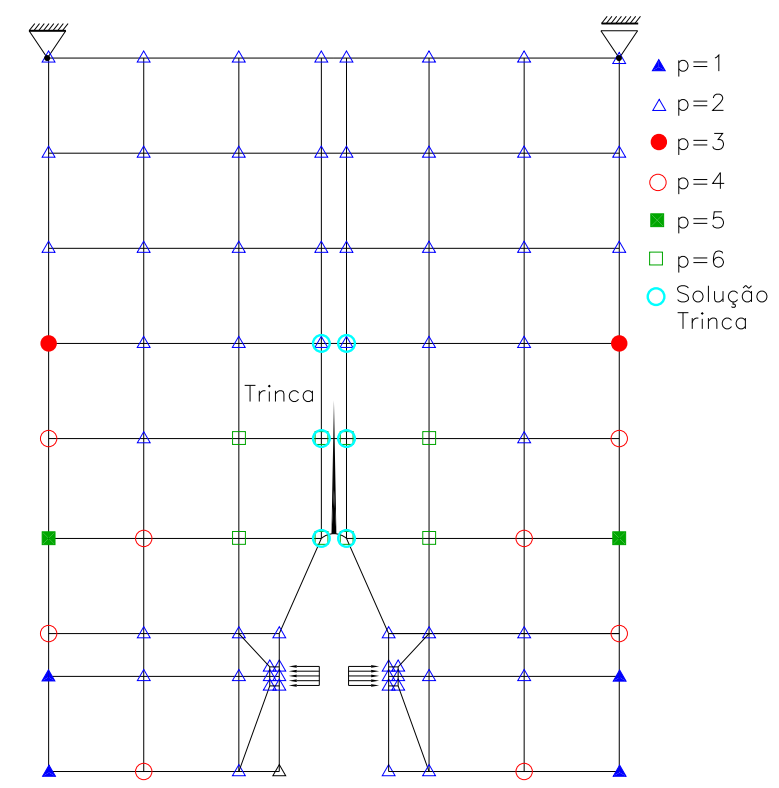

Figura 7.1: Malha e condições de contorno utilizadas para a análise

Nesse contexto, o MEFG pode ser uma importante ferramenta de análise numérica, devido à versatilidade no enriquecimento da aproximação. Para ilustrar essa afirmação, seja a malha exibida na Figura 7.1. Além do refinamento polinomial, determinado pela análise adaptativa apresentada na seção 6.7.2, estão também indicadas as nuvens em que a PU é enriquecida por funções especiais. Estas funções, $\left\{{ }^{\alpha} u_{x},{ }^{\alpha} u_{y}\right\}_{\alpha=1}^{\infty}$, 
definidas como uma série infinita em elasticidade bi-dimensional, correspondem ao campo de deslocamentos em pontos na vizinhança da extremidade da trinca e distantes do contorno, SZABÓ; BABUŠKA (1991). Do apêndice J, pode-se chegar à seguinte aproximação para os deslocamentos, utilizando-se apenas os primeiros termos da série correspondentes ao modo I de fratura:

$$
\begin{aligned}
& \tilde{u}_{x}(\boldsymbol{x})=\sum_{j=1}^{N} N_{j}(\boldsymbol{x})\left\{u_{j}^{x}+\sum_{i=1}^{q_{j}(p)} p_{i} b_{j i}^{x}+\left[1-\frac{{ }^{1} u_{x}^{(1)}(\boldsymbol{x})}{{ }^{1} u_{x}^{(1)}\left(\boldsymbol{x}_{j}\right)}\right] d_{j}^{x}\right\} \\
& \tilde{u}_{y}(\boldsymbol{x})=\sum_{j=1}^{N} N_{j}(\boldsymbol{x})\left\{u_{j}^{y}+\sum_{i=1}^{q_{j}(p)} p_{i} b_{j i}^{y}+\left[1-\frac{{ }^{1} u_{y}^{(1)}(\boldsymbol{x})}{{ }^{1} u_{y}^{(1)}\left(\boldsymbol{x}_{j}\right)}\right] d_{j}^{y}\right\}
\end{aligned}
$$

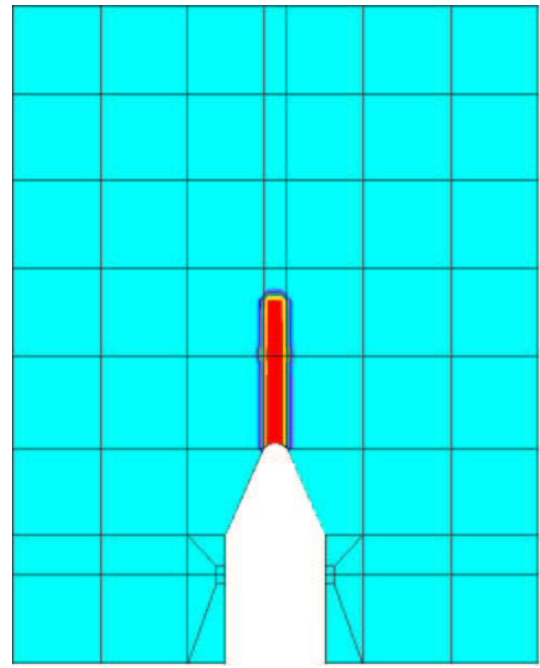

(a) Distribuição do dano

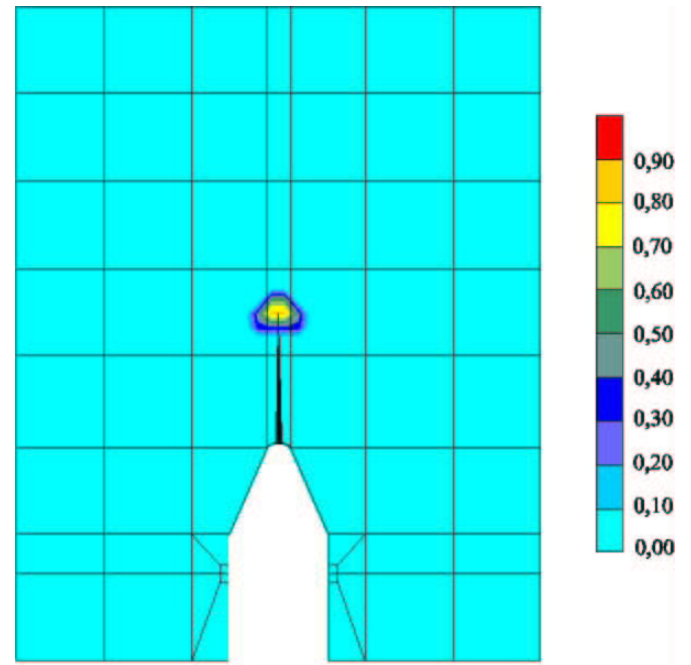

(b) Aproximação adotada - análise com trinca

Figura 7.2: Confrontação dano $\times$ fratura

Essa aproximação pode ser introduzida durante a análise, por exemplo quando o nível de força atingir $18,5 \mathrm{kN}$, o que equivale a substituir a região danificada por uma fratura macroscópica. Para isso torna-se necessário estabelecer o tamanho da trinca a ser considerada. Nesse experimento numérico, foi adotado um comprimento de $14 \mathrm{~cm}$ que aproximadamente corresponde ao tamanho da região de dano, Figuras 7.2(a) e 7.2(b). Uma análise elástica foi então realizada, encontrando-se o valor de 0,186 mm para a abertura do entalhe. Este resultado difere em 1,1\% ao encontrado para a análise discutida na seção 6.7.2 (0,184 mm).

Os resultados apresentados demonstram a possibilidade de se usar o enriqueci- 
mento com funções não-polinomiais para a simulação da transição entre dano e fratura. Não se quer afirmar, contudo, que o procedimento de análise adotado seja o ideal para tal objetivo. Um ponto fundamental consiste em se estabelecer um critério para indicar a equivalência entre uma certa distribuição de dano e uma fratura única. Esse critério serviria para determinar o nível real de força em que novo enriquecimento deve ser realizado sobre a aproximação e a dimensão correta para a trinca. A partir desse ponto, a propagação da trinca pode ser conduzida considerando-se a existência de uma zona de processo à frente de sua extremidade. Nessa zona de processo o fenômeno dominante corresponderia à formação e evolução do dano, Figura 7.3. A danificação do material provocaria, portanto, o abatimento no campo de tensões junto à ponta da trinca eliminando a singularidade desse. Por essa razão, as funções enriquecedoras empregadas em (7.1) e (7.2) precisariam ser outras, exigindo assim um estudo mais aprofundado nesse sentido.

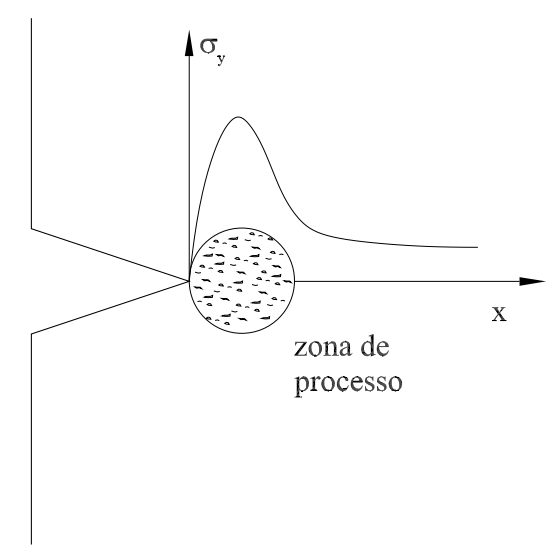

Figura 7.3: Zona de processo de danificação

Ainda que bastante simplificado, este exemplo demonstra as potencialidades do MEFG para a análise de problemas em que dano e fratura são considerados. Em primeiro lugar, existe a possibilidade de serem introduzidas funções não-polinomiais, próprias para a simulação dos fenômenos analisados. Ao contrário das formulações clássicas do MEF, a mudança na aproximação é bastante simples de ser implementada no MEFG, permitindo-se que o enriquecimento possa ser empregado a partir do instante em que for necessário, ou seja, com o surgimento da fratura. Sua propagação pode ser facilmente simulada, bastando para isso que novos enriquecimentos sejam realizados sobre a PU das nuvens atravessadas pela trinca. 


\subsection{Outras Propostas de Futuros Desenvolvimentos}

Além da simulação numérica da fase de transição entre dano e fratura, diversas outras questões surgiram de observações suscitadas pelas análises realizadas neste trabalho e podem ser sugeridas como temas para desenvolvimentos futuros:

Integração numérica: em todos os exemplos de análise não-linear, foi empregado um número razoavelmente grande de pontos de Gauss para que assim fosse possível representar, no cálculo da matriz de rigidez, a variação das propriedades físicas do material. Além disso, no cálculo da deformação equivalente pela abordagem não-local, parte-se do princípio que o somatório indicado pela relação (4.23) reproduza, de forma suficientemente precisa, uma integração na região delimitada pelo raio $r_{\mathrm{n} l}$. Esta hipótese será mais próxima de ser verificada à medida que um maior número de pontos de integração sejam incluídos ao domínio. Sugere-se, portanto, um estudo aprofundado a esse respeito, procurando-se indentificar a influência da ordem da quadratura na determinação da distribuição do dano e na correta aplicação de modelos não-locais. Ainda com relação à integração numérica uma importante questão a ser investigada consiste na implementação de regras de quadraturas mais adequadas à integração de funções não-polinomiais, especialmente aquelas com singularidades como é o caso das funções (7.1) e (7.2).

Localização: no exemplo da seção 5.4 foi mostrado que a técnica de enriquecimento polinomial do MEFG pode ser usada com sucesso na descrição de problemas em que ocorre a concentração do dano em uma pequena região do domínio. Abre-se assim a possibilidade de aplicação do MEFG aos problemas com localização de deformações onde novas funções não-polinomiais, inclusive, possam ser utilizadas com vantagens. Esta poderia ser, aliás uma interessante alternativa no tema da transição entre o dano e fratura e, para isso, sugere-se como leitura o trabalho de MANZOLI; OLIVER; CERVERA (1998).

Análise não-linear adaptativa: apesar de sugerida no apêndice I, a estratégia de transferência das variáveis, como parte do procedimento adaptativo, não foi implementada. O emprego dessa técnica permite não apenas a variação no número de pontos de Gauss à medida que o refinamento é realizado ao longo do processo de solução incremental, como também a utilização da quadratura de GaussLegendre. A função suavizada para o estado de danificação obtida por meio 
dessa técnica também pode ser empregada para se determinar o dano na face de cada elemento, necessário para o cálculo das funções indicadoras de erro.

Indicador de erro: em lugar de se definir o problema (6.33) considerando-se o domínio do elemento, o mesmo poderia ser construído tomando-se isoladamente cada nuvem $\omega_{j}$. Dessa maneira, as funções de erro locais passariam a ser construídas como:

$$
\tilde{\boldsymbol{e}}_{p}^{\omega_{j}} \stackrel{\text { def }}{=}\left(\boldsymbol{\Phi}_{p+1}^{0}\right)_{\omega_{j}}^{T} \boldsymbol{I}_{\omega_{j}}
$$

onde apenas as funções de forma $\left(\boldsymbol{\Phi}_{p+1}^{0}\right)_{\omega_{j}}^{T}$ e parâmetros $\boldsymbol{I}_{\omega_{j}}$ associados à nuvem $\omega_{j}$ estariam presentes. O novo indicador seria obtido pela seguinte expressão:

$$
\left\|\tilde{\boldsymbol{e}}_{p}^{\omega_{j}}\right\|_{u\left(\omega_{j}\right)}=\left[\mathcal{B}_{\omega_{j}}\left(\tilde{\boldsymbol{e}}_{p}^{\omega_{j}}, \tilde{\boldsymbol{e}}_{p}^{\omega_{j}}\right)\right]^{1 / 2}
$$

Para se determinar o estimador de erro global, as funções locais poderiam ser "costuradas" pela sua multiplicação pelas funções de PU, a exemplo do que é feito na estratégia para a transferência de variáveis no apêndice I. Dessa forma, a função de erro empregada para se ter o estimador global, segundo (6.4), seria definida como:

$$
\tilde{\boldsymbol{e}}_{p}=\sum_{j=1}^{N} \mathcal{N}_{j} \tilde{\boldsymbol{e}}_{p}^{\omega_{j}}
$$

Tal estratégia para a estimativa do erro tem a vantagem de fornecer indicadores diretamente associados aos nós, sobre os quais o refinamento polinomial é realizado. A questão fundamental a ser resolvida corresponde, então, à formulação de um problema equivalente ao representado pelo (6.33) para cada nuvem. Deve-se, todavia, lembrar que o campo de tensões não é contínuo entre elementos e, por essa razão surgem saltos nas tensões no interior das nuvens. Uma alternativa seria empregar funções de Shepard com continuidade $C^{1}$ na PU, o que produziria uma definição única para as tensões em todo o domínio.

Refinamento $s$ : para a análise adaptativa não-linear sugere-se introduzir o refinamento $s$ proposto em FISH (1992) e FISH (1994), em que, de maneira hierárquica, diversos níveis de malha são sobrepostos à malha original. $\mathrm{O}$ critério para conduzir tal refinamento seria governado pelo surgimento do dano. Dessa forma, se em uma determinada nuvem o processo de danificação fosse iniciado, uma nova malha deveria ser aninhada aos elementos dessa nuvem. Tal estratégia 
teria o objetivo de descrever, com maior exatidão, a distribuição do dano antes do cálculo do estimador de erro e aplicação do refinamento polinomial.

Estimador em análise não-linear: para estimar o erro em análise não-linear poderia ser utilizada a proposta de CIRAK; RAMM (2000), cujo o desenvolvimento inicial é semelhante ao discutido na seção (6.6.1) mas que emprega a versão explícita associada às técnicas de pós-processamento dos gradientes da solução. Além disso, o problema do erro é formulado em abordagem dual, obtendo-se um estimador associado não à norma energia mas a uma variável, que pode ser, por exemplo, a tensão. O maior problema corresponderia ao pós-processamento dos gradientes da solução que, normalmente, não é indicado para análises em que a aproximação é de elevada ordem polinomial. Por outro lado, no trabalho de BARTELS; CARSTENSEN (2000) mostra-se que é possível empregar tensões suavizadas no cálculo do erro para aproximações com ordem polinomial elevada, o que viabilizaria o procedimento $p$-adaptativo.

Todas as sugestões apresentadas são, em primeira análise, caminhos viáveis a serem pesquisados. Ainda que na forma proposta possam não ser plenamente realizáveis, acredita-se que estabeleçam direções para futuros trabalhos relacionados com o tema desta tese. 


\section{Referências Bibliográficas}

AINSWORTH, M.; ODEN, J. T. A posteriori error estimation in finite element analysis. Computer Methods in Applied Mechanics and Engineering, v. 142, p. 1-88, 1997.

ÁLVARES, M. S. Estudo de um modelo de dano para o concreto: Formulação, identificação paramétrica e aplicação com o emprego do método dos elementos finitos. Dissertação (Mestrado) - Escola de Engenharia de São Carlos - Universidade de São Paulo, 1993.

AMARATUGA, K.; WILLIAMS, J. R. Wavelet-Galerkin solution of boundary value problems. Archives of Computational Methods in Engineering, v. 4, n. 3, p. 243-285, 1997.

ARGYRIS, J.; MLEJNEK, H. P. Dynamics of structures. [S.1.]: North-Holland, 1991.

ASSAN, A. E. Método dos Elementos Finitos: Primeiros Passos. [S.1.]: Editora Unicamp, 1999. ISBN 85-268-0475-8.

BABUŠKA, I.; CALOZ, G.; OSBORN, J. E. Special finite element method for a classe of second order elliptic problems whith rough coefficients. SIAM Journal on Numerical Analysis, v. 31, n. 4, p. 745-981, 1994.

BABUŠKA, I.; MELENK, J. M. The partition of unity method. International Journal for Numerical Methods in Engineering, v. 40, p. 727-758, 1997.

BABUŠKA, I.; RHEINBOLDT, W. C. Error estimates for adaptive finite element method computations. SIAM Journal for Numerical Analysis, v. 15, n. 736-753, 1978.

BABUŠKA, I.; RHEINBOLDT, W. C. A posteriori error estimates for the finite element method. International Journal for Numerical Methods in Engineering, v. 12, n. $1597-1615,1978$. 
BABUŠKA, I.; RHEINBOLDT, W. C. Adaptive approaches and reliability estimations in finite element analysis. Computer Methods in Applied Mechanics and Engineering, v. 17/18, p. 519-540, 1979.

BABUŠKA, I. et al. A-posteriori error estimation for finite element and generalized finite element method. Internet report. Novembro 1997. Disponível em: $<$ http://yoyodyne.tamu.edu/research/GFEM/index.html $>$. Acesso em: Outubro de 1999.

BABUŠKA, I. et al. A model study of the quality of a posteriori estimators of linear elliptic problems. Part Ia: Error estimation in the interior of patchwise uniform grids of triangles. Computer Methods in Applied Mechanics and Engineering, v. 114, n. 307-378, 1994.

BABUŠKA, I. et al. Validation of a-posteriori error estimators by numerical approach. International Journal for Numerical Methods in Engineering, v. 37, p. 10731123, 1994.

BANK, R. E.; WEISER, A. Some a posteriori error estimators for elliptic partial defferential equations. Mathematics Computations, v. 44, p. 283-301, 1985.

BARTELS, S.; CARSTENSEN, C. Each averaging technique yields reliable a posteriori error control in FEM on unstructured grids. part ii: Higer order FEM. Internet report - Christian-Albrechts-Universität. Janeiro 2000. Disponível em: $<$ http://www.numerik.uni-kiel.de/reports/authors.html\#Carstensen / $>$. Acesso em: Maio de 2000.

BATHE, K. J. Finite element procedures. [S.1.]: Prentice-Hall, Inc., 1996. ISBN 0-13-301458-4.

BAZǍNT, Z. P.; PIJAUDIER-CABOT, G. Measurement of characteristic length of nonlocal continuum. Journal of Engineering Mechanics, ASCE, v. 115, p. 755-767, 1989.

BELYTSCHKO, T.; BLACK, T. Elastic crack growth in finite elements with minimal remeshing. International Journal for Numerical Methods in Engineering, v. 45, p. 601-620, 1999.

BELYTSCHKO, T. et al. Meshless methods: An overview and recent developments. Computer Methods in Applied Mechanics and Engineering, v. 139, p. 3-47, 1996. 
BELYTSCHKO, T.; LU, Y.; GU, L. Element-free Galerkin methods. International Journal for Numerical Methods in Engineering, v. 37, p. 229-256, 1994.

BELYTSCHKO, T.; LU, Y. Y.; GU, L. Crack propagation by element free Galerkin methods. Advanced Computational Methods for Material Modelling - ASME, v. 268, p. 191-205, 1993.

BLACKER, T.; BELYTSCHKO, T. Superconvergent patch recovery with equilibrium and conjoint interpolant enhancements. International Journal for Numerical Methods in Engineering, v. 37, n. 517-536, 1994.

BOROOMAND, B.; ZIENKIEWICZ, O. C. An improved REP recovery and the effectivity robustness test. International Journal for Numerical Methods in Engineering, v. 40, n. 3247-3277, 1997.

BOROOMAND, B.; ZIENKIEWICZ, O. C. Recovery procedures in error estimation anda adaptivity Part I: Adaptivity in nonlinear problems of elasto-plasticity behaviour. Computer Methods in Applied Mechanics and Engineering, v. 176, p. 127-146, 1999.

CARSTENSEN, C.; ALBERTY, J. Averaging techniques for reliable a posteriori fe-error control in elastoplasticity with hardening. Internet report - ChristianAlbrechts-Universität. Setembro 2000. Disponível em: <http://www.numerik.unikiel.de/reports/authors.html\#Carstensen />. Acesso em: Maio de 2000.

CARSTENSEN, C.; FUNKEN, S. A. Averaging technique for FE-a posteriori error control in elasticity. part i: Conforming FEM. Internet report - ChristianAlbrechts-Universität. Fevereiro 2000. Disponível em: <http://www.numerik.unikiel.de/reports/authors.html\#Carstensen />. Acesso em: Maio de 2000.

CARSTENSEN, C.; FUNKEN, S. A. Each averaging technique yields reliable a posteriori error control in FEM on ustructured grids. part ii: higher order FEM. Internet report - Christian-Albrechts-Universität. Janeiro 2000. Disponível em: $<$ http://www.numerik.uni-kiel.de/reports/authors.html\#Carstensen / $>$. Acesso em: Maio de 2000.

CHEN, W. F.; HAN, D. J. Plasticity for Structural Engineers. New York: SpringerVerlag, 1988. 
CIRAK, F.; RAMM, E. A posteriori error estimation and adaptivity for elastoplasticity using the reciprocal theorem. International Journal for Numerical Methods in Engineering, v. 47, p. 379-393, 2000.

COMI, C. A nonlocal damage modelo with permanent strains for quasi-brittle materials. In: BENALLAL, A. (Ed.). Continuous Damage and Fracture. Paris: Elsevier, 2000. p. 221-231.

CORDES, L.; MORAN, B. Treatment of material discontinuidty in the element-free galerkin method. Computer Methods in Applied Mechanics and Engineering, v. 139, p. 75-89, 1996.

DAVENNE, L.; SAOURIDIS, C.; PIAU, J. M. Un code de calcul pour la prévision du comportement de structures endommageables en béton, en béton armé ou en béton de fibres. [S.1.], Novembro 1989. 478. Série: Béton 267.

DEMKOWICZ, L. et al. Toward a universal h-p adaptive finite element strategy, part 1. constrained approximation and data structure. Computer Methods in Applied Mechanics and Engineering, v. 77, p. 79-112, 1989.

DEMKOWICZ, L.; ODEN, J. T.; STROUBOULIS, T. Adaptive finite elements for flow problems with moving boundaries. part 1: Variational principles and a posteriori error estimates. Computer Methods in Applied Mechanics and Engineering, v. 46, p. 217-251, 1984.

DOLBOW, J.; BELYTSCHKO, T. An introduction to programming the meshless element free Galerkin method. Archives of Computational Methods in Engineering, v. 5, p. 207-241, 1998.

DOLBOW, J.; MOËS, N.; BELYTSCHKO, T. Modeling Fracture in MindlinReissner Plates with the eXtended Finite Element method. to appear, 2000.

DOLBOW, J. E. Numerical Integration in Meshfree Methods. Dissertação (Mestrado) — Northwestern University, http://tam6.mech.nwu.edu/jed/res2.html, Maio 1998.

DOLBOW, J. E. An Extended Finite Element Method with Discontinuous Enrichment for Applied Mechanics. Tese (Doutorado) - Northwestern University, http://tam6.mech.nwu.edu/jed/res2.html, Dezembro 1999. 
DUARTE, C. A. Estudo da versão p do método dos elementos finitos para problemas da elasticidade e de potencial. Dissertação (Mestrado) - Departamento de Engenharia Mecânica da Universidade Federal de Santa Catarina, 1991.

DUARTE, C. A. A Review of Some Meshless Methods to Solve Partial Differencial Equations. [S.1.], 1995. Technical Report 06.

DUARTE, C. A. The hp-cloud method. Tese (Doutorado) - The University of Texas at Austin, Dezembro 1996.

DUARTE, C. A.; BABUŠKA, I.; ODEN, J. Generalized finite element methods for three-dimensional structural mechanics problems. Computers \& Structures, v. 77, n. 2, p. 215-232, 2000.

DUARTE, C. A. et al. A generalized finite element method for the simulation of threedimensional dynamic crack propagation. Computer Methods in Applied Mechanics and Engineering, v. 190, p. 2227-2262, 2001.

DUARTE, C. A.; ODEN, J. T. Hp Clouds - A Meshless Method to Solve BoundaryValue Problem. [S.1.], Maio 1995. Technical Report.

DUARTE, C. A.; ODEN, J. T. An h-p adaptive method using cloud. Computer Methods in Applied Mechanics and Engineering, v. 139, p. 237-262, 1996.

DUARTE, C. A.; ODEN, J. T. H-p Clouds-an h-p Meshless Method. In: Numerical Methods for Partial Differential Equations. [S.1.]: John Wiley \& Sons, Inc., 1996. p. $1-34$.

DUFF, I. S.; REID, J. K. The multifrontal solution of indefinite sparse symmetric linear systems. ACM Transations on Mathematical Software, v. 9, n. 3, p. 302-325, 1983.

DVORKIN, E. N.; CUITIÑO, A. M.; GIOIA, G. Finite elements with displacement interpolated embedded localization lines insensitive to mesh size and distortions. International Journal for Numerical Methods in Engineering, v. 30, p. 541-564, 1990.

ESPELID, T. O. On integration vertex singularities using extrapolation. BIT, v. 34, p. 62-79, 1994. 
FISH, J. The s-version of the finite element method. Computers \& Structures, v. 43, n. 3, p. 539-547, 1992.

FISH, J. On adaptive multilevel superposition of finite element meshes for linear elastostatics. Applied Numerical Mathematics, v. 14, p. 135-164, 1994.

GALLIMARD, L.; LADEVÈZE, P.; PELLE, J. P. Error estimation and adaptivity in elastoplasticity. International Journal for Numerical Methods in Engineering, v. 39, p. $189-217,1996$.

GALLIMARD, L.; LADEVÈZE, P.; PELLE, J. P. An enhanced error estimator on the constitutive relation for plasticity problems. Computers \& Structures, n. 78, p. 801-810, 2000.

GORDON, W. J.; HALL, C. A. Construction of curvilinear co-ordinate systems and applications to mesh generation. International Journal for Numerical Methods in Engineering, v. 7, p. 461-477, 1973.

HEGEN, D. An Element-free Galerkin Method for Crack Propagation in Brittle Materials. Tese (Doutorado) — Faculty of Mechanical Engineering of Eindhoven University of Technology, 1997.

HILLER, J. F.; BATHE, K. J. On higher-order-accuracy points in isoparametric finite element analysis and an application to error assessment. Computers \& Structures, v. 79, p. 1275-1285, 2001.

HSL. Hsl archive. 2000. Disponível em: < http://www.cse.clrc.ac.uk/Activity/HSL>. Acesso em: Outubro de 2000.

JOHNSON, C.; HANSBO, P. Adaptive finite element methods in computational mechanics. Computer Methods in Applied Mechanics and Engineering, v. 101, p. 143-181, 1992.

KRAJCINOVIC, D.; LEMAITRE, J. Continuum Damage Mechanics - Theory and Applications. [S.1.]: Springer-Verlag, 1987.

KRONGAUZ, Y.; BELYTSCHKO, T. EFG approximation with discontinuous derivatives. International Journal for Numerical Methods in Engineering, v. 41, p. 1215-1233, 1998. 
KRYSL, P.; BELYSTSCHKO, T. Element-free Galerkin method: Convergence of the continuous and discontinuous shape functions. Computer Methods in Applied Mechanics and Engineering, v. 148, n. 3-5, p. 257-277, Setembro 1997.

LA BORDERIE, C. Phenomenes unilateraux dans un materiau endommageable: modelisation et application a l'analyse de structures en beton. Tese (Doutorado) Université Pierre et Marie Curie - Paris 6, 1991.

LADEVÈZE, P.; MAUNDER, E. A. W. A general method for recovering equilibrating element tractions. Computer Methods in Applied Mechanics and Engineering, v. 137, p. 111-151, 1996.

LANCASTER, P.; SALKAUSKAS, K. Surfaces generated by moving least squares methods. Mathematics of Computation, v. 37, n. 155, p. 141-158, Julho 1981.

LEE, N. S.; BATHE, K. J. Effects of element distortions on the performance of isoparametric elements. International Journal for Numerical Methods in Engineering, v. 36, p. 3553-3576, 1993.

LEMAITRE, J. A Course on Damage Mechanics. [S.1.]: Springer-Verlag, 1992.

LEMAITRE, J.; CHABOCHE, J. L. Mechanics of solid materials. [S.1.]: Cambridge University Press, 1990.

LIU, W. K. et al. Reproducing kernel particles methods for structural dynamics. International Journal for Numerical Methods in Engineering, v. 8, p. 1655-1679, 1995.

LYRA, P. R. M. Uma Estratégia de Refinamento Auto-Adaptativo Versão $h$ do Método dos Elementos Finitos Aplicada a Problemas Bi-dimensionais Regidos pela Equação de Campo. Tese (Doutorado) — COPPE/UFRJ, 1988.

MALVERN, L. E. Introduction to the Mechanics of a Continuous Medium. [S.1.]: Prentice-Hall, Inc., 1969.

MANZOLI, O.; OLIVER, X.; CERVERA, M. Localización de Deformaciones: Análisis y Simulación Numérica de Discontinuidades en Mecánica de Sólidos. [S.1.], 1998. 44. Monografia. 
MAZARS, J. Application de la mécanique de l'endommagement au comportement non lineaire et à la rupture du béton de structure. Tese (Doutorado) - Université Paris 6, 1984.

MAZARS, J.; PIJAUDIER-CABOT, G. Damage localization analyzed as a crack propagation? In: BAZǍNT, Z. P. et al. (Ed.). Fracture and Damage in Quasibrittle Structures: Experiment, modelling and computer analysis. London: E\&FN Spon, 1994. p. $145-157$.

MELENK, J. M. Finite Element Methods with harmonic shape functions for solving Laplace's equation. Dissertação (Mestrado) - University of Maryland, College Park, 1992.

MELENK, J. M. On Generalized Finite Element Methods. Tese (Doutorado) University of Maryland, College Park, 1995.

MELENK, J. M.; BABUŠKA, I. The partition of unity finite element method: Basic theory and applications. Computer Methods in Applied Mechanics and Engineering, v. 39, p. 289-314, 1996.

MENDONÇA, P. T. R.; BARCELLOS, C. S.; DUARTE, A. Investigations on the hpcloud method by solving Timoshenko beam problems. Computational Mechanics, v. 25, p. 286-295, 2000.

MONAGHAN, J. J. Why particle methods work. SIAM Journal on Scientific and Statistical Computing, v. 3, p. 422-433, 1982.

MONAGHAN, J. J. Simulating free surface flows with sph. Journal of Computational Physics, v. 110, p. 399-406, 1994.

MOËS, N.; DOLBOW, J.; BELYTSCHKO, T. A finite element method for crack growth without remeshing. International Journal for Numerical Methods in Engineering, v. 46, p. 131-150, 1999.

NAYROLES, B.; TOUZOT, G.; VILLON, P. Generalizing the finite element method: Diffuse approximation and diffuse elements. Computational Mechanics, v. 10, p. 307-318, 1992.

OÑATE, E.; IDELSOHN, S.; ZIENKIEWICZ, O. C. Finite Point Methods in Computational Mechanics. [S.1.], Julho 1995. Report 67. 
OÑATE, E. et al. A finite point method in computational mechanics. applications to to convective transport and fluid flow. International Journal for Numerical Methods in Engineering, v. 39, p. 3839-3866, 1996.

OÑATE, E. et al. A stabilized finite point method for analysis of fluid mechanics problems. Computer Methods in Applied Mechanics and Engineering, v. 139, p. 315-346, 1996.

ODEN, J. T.; BRAUCHLI, H. J. On the calculation of consistent stress distribuitions in finite element applications. International Journal for Numerical Methods in Engineering, v. 3, p. 317-325, 1971.

ODEN, J. T. et al. Toward a universal h-p adaptive finite element strategy, part 2. a posteriori error estimation. Computer Methods in Applied Mechanics and Engineering, v. 77, p. 113-180, 1989.

ODEN, J. T.; DUARTE, C. A. Clouds, cracks and fem's. In: REDDY, B. D. (Ed.). Recent Developments in Computational and Applied Mechanics. Barcelona, Spain: International Center for Numerical Methods in Engineering, CIMNE, 1997. p. 302321.

ODEN, J. T.; DUARTE, C. A. Solution of singular problems using hp clouds. In: WHITEMAN, J. R. (Ed.). The Mathematics of Finite Elements and Approximations. New York: John Wiley \& Sons, 1997. p. 35-54.

ODEN, J. T.; DUARTE, C. A.; ZIENKIEWICZ, O. C. A New Cloud-Based hp Finite Element Method. Austin, Texas, USA, Dezembro 1996.

ODEN, J. T.; DUARTE, C. A.; ZIENKIEWICZ, O. C. A new cloud-based hp finite element method. Computer Methods in Applied Mechanics and Engineering, v. 153, p. 117-126, 1998.

ODEN, J. T.; REDDY, J. N. An Introduction to the Mathematical Theory of Finite Elements. [S.1.]: John Willey \& Sons, Inc., 1976. (Pure and Applied Mathematics). ISBN 0-471-65261-X.

OHTSUBO, H.; KITAMURA, M. Element by element a posteriori error estimation and improvement of stress solutions for two-dimensional elastic problems. International Journal for Numerical Methods in Engineering, v. 29, p. 223-244, 1990. 
OHTSUBO, H.; KITAMURA, M. Element by element a posteriori error estimation of the finite element analysis for three-dimensional elastic problems. International Journal for Numerical Methods in Engineering, v. 33, p. 1755-1769, 1992.

OLIVEIRA, D. S. Aplicação do Método de Rayleigh-Ritz na Integração Numérica das Equações da Técnica do Meio-Contínuo. Dissertação (Mestrado) - Universidade de São Paulo, Escola de Engenharia de São Carlos, Departamento de Estruturas, 1982.

OLIVER, J. Continuum modelling of strong discontinuties in solid mechanics using damage models. Computational Mechanics, v. 17, p. 49-61, 1995.

ORTIZ, M.; LEROY, Y.; NEEDLEMAN, A. A finite element method for localized failure analysis. Computer Methods in Applied Mechanics and Engineering, v. 61, p. 189-214, 1987.

ORTIZ, M.; QUIGLEY, IV, J. J. Adaptive mesh refinement in strain localization problems. Computer Methods in Applied Mechanics and Engineering, v. 90, p. 781804, 1991.

PAULA, C. F. Contribuição ao estudo das Respostas Numéricas Não-Lineares Estática e Dinâmica de Estruturas Reticuladas Planas. Tese (Doutorado) - Universidade de São Paulo, Escola de Engenharia de São Carlos, Departamento de Estruturas, Abril 2001.

PEREGO, M. A. Danneggiamento dei Materiali Lapidei: Leggi Costitutive, Analisi per Element Finiti ed Applicazioni. Tese (Doutorado) - Facoltà di Ingegneria Politecnico di Milano, 1989.

PERIĆ, D.; YU, J.; OWEN, D. R. J. On error estimates and adaptivity in elastoplastic solids: applications to the numerical simulation of strain localization in classical and cosserat continua. International Journal for Numerical Methods in Engineering, v. 37, p. 1351-1379, 1994.

PINTO, C. et al. Study of error components in finite element analysis of non-linear structural problems. In: Proceedings of the European Congress on Computational Methods in Applied Sciences and Enginerring - ECCOMAS. Barcelona: [s.n.], 2000. 
PITUBA, J. J. C.; PROENÇA, S. P. B.; ÁlVARES, M. S. Estudo do desempenho de modelos de dano para estruturas reticulares em concreto armado. Computational Methods in Engineering'99, 1999.

PROENÇA, S. P. B. Meccanica del Danneggiamento di Materiali e Strutture: Applicazioni al Calcestruzzo. [S.1.], 1991. Publicação resultante de pesquisas realizadas.

RAMM, E.; CIRAK, F. Adaptivity for nonlinear thin-walled structures. In: OWEN, D. R. J.; OÑATE, E.; HINTON, E. (Ed.). COMPUTIONAL PLASTICITY: Fundamentals and applications. Barcelona: CIMNE, 1997. p. 145-163.

RANJBARAN, A. Embedding of reinforcements in reinforced concrete elements implemented in DENA. Computers \& Structures, v. 40, n. 4, p. 925-930, 1991.

RANNACHER, R.; SUTTMEIER, F. T. A posteriori error estimation and mesh adaptation for finite element models in elasto-plasticity. Computer Methods in Applied Mechanics and Engineering, v. 176, p. 333-361, 1999.

RHEINBOLDT, W. C. Error estimates for nonlinear finite element computations. Computers \& Structures, v. 20, n. 1-3, p. 91-98, 1985.

RIVLIN, T. J. An Introduction to the Approximation of Functions. [S.1.]: Blaisdell Publishing Company, 1969.

RODRIGUES, R. O. Análise dinâmica bidimensional não-linear Física e Geométrica. Tese (Doutorado) - School of Engineering, University of São Paulo, 1997.

SAVASSI, W. Aplicação do método dos elementos finitos aos edifícios altos. In: XVII Jornadas Sudamericanas de Ingeniería Estructural: Simposio panamericano de estructuras. Caracas: [s.n.], 1975. p. 1-4.

SHEPARD, D. A two-dimensional function for irregualarly spaced data. In: $A C M$ National Conference. [S.1.: s.n.], 1968. p. 517-524.

Stamato, M. C. Associação Contínua de Painéis de Contraventamento. [S.1.], 1972. Relatório 157.

STOLARSKA, M. et al. Modelling crack growth by level sets in extended finite element method. International Journal for Numerical Methods in Engineering, v. 51, p. 943-960, 2001. 
STROUBOULIS, T.; BABUŠKA, I.; COPPS, K. The design and analysis of the generalized finite element method. Computer Methods in Applied Mechanics and Engineering, v. 181, n. 1-3, p. 43-69, Janeiro 2000.

STROUBOULIS, T.; COPPS, K.; BABUŠKA, I. The generalized finite element method. Computer Methods in Applied Mechanics and Engineering, v. 190, p. 40814193, 2001.

STROUBOULIS, T.; HAQUE, K. A. Recent experiences with error estimation and adaptivity, Part i: Review of error estimators for scalar elliptic problems. Computer Methods in Applied Mechanics and Engineering, v. 97, p. 399-436, 1992.

SZABÓ, B.; BABUŠKA, I. Finite Element Analysis. [S.1.]: Johwn Wiley \& Sons, Inc., 1991.

SZABÓ, B. A.; BABUŠKA, I.; CHAYAPATHY, B. K. Stress computations for nearly incompressible materials by the p-version of the finite element method. International Journal for Numerical Methods in Engineering, v. 28, p. 2175-2190, 1989.

TIMOSHENKO, S. P.; GOODIER, J. N. Teoria da Elasticidade. [S.1.]: Guanabara Dois S. A., 1980.

W. K. LIU, S. J.; ZHANG, F. Y. Reproducing kernel particle methods. International Journal for Numerical Methods in Engineering, v. 20, p. 1081-1106, 1995.

ZIENKIEWICZ, O. C.; MORGAN, K. Finite Elements and Approximation. [S.1.]: John Willey \& Sons, Inc., 1983.

ZIENKIEWICZ, O. C.; ZHU, Z. A simple error estimator and adaptive procedure for practical engineering analysis. International Journal for Numerical Methods in Engineering, v. 24, p. 337-357, 1987.

ZIENKIEWICZ, O. C.; ZHU, Z. The superconvergent patch recovery and a posteriori error estimates. Part 1: The recovery technique. International Journal for Numerical Methods in Engineering, v. 33, p. 1331-1364, 1992.

ZIENKIEWICZ, O. C.; ZHU, Z. The superconvergent patch recovery and a posteriori error estimates. Part 2: Error estimates and adaptivity. International Journal for Numerical Methods in Engineering, v. 33, p. 1365-1382, 1992. 


\section{Apêndice A}

\section{Formulação Tangente para o Modelo de Mazars}

Para se obter a formulação tangente para o modelo de Mazars, equação (4.6), é necessário que se determine a lei de evolução do dano $\dot{D}$, derivando-se no tempo as expressões presentes no modelo de Mazars:

$$
\dot{D}=\left(\frac{\partial D}{\partial \varepsilon_{e q}} \frac{\partial \varepsilon_{e q}}{\partial \varepsilon_{i}}+\frac{\partial D}{\partial \varepsilon_{i}}\right) \frac{\partial \varepsilon_{i}}{\partial t}=\boldsymbol{H} \cdot \dot{\boldsymbol{\varepsilon}}
$$

onde:

- $\boldsymbol{H} \stackrel{\text { def }}{=}\left(\frac{\partial D}{\partial \varepsilon_{e q}} \frac{\partial \varepsilon_{e q}}{\partial \varepsilon_{i}}+\frac{\partial D}{\partial \varepsilon_{i}}\right)$ é um vetor que representa a derivada da variável dano com relação às deformações principais;

- $\dot{\varepsilon}$ é o vetor das taxas de variação das deformações principais com o tempo.

Observe que o índice $i$ refere-se às direções principais, além de se comportar como índice mudo. Como os coeficientes $\alpha_{C}$ e $\alpha_{T}$ são funções do estado de deformação, enquanto que $D_{T}$ e $D_{C}$ são definidos a partir da deformação equivalente, a expressão acima pode ser reescrita da seguinte forma:

$$
\dot{D}=\left[\mathcal{F}\left(\varepsilon_{e q}\right) \frac{\partial \varepsilon_{e q}}{\partial \varepsilon_{i}}+\mathcal{G}\left(\varepsilon_{i}\right)\right] \frac{\partial \varepsilon_{i}}{\partial t}
$$

sendo $\mathcal{F}\left(\varepsilon_{e q}\right)$ e $\mathcal{G}\left(\varepsilon_{i}\right)$ funções definidas como:

$$
\begin{gathered}
\mathcal{F}\left(\varepsilon_{e q}\right)=\alpha_{T} \mathcal{F}_{T}\left(\varepsilon_{e q}\right)+\alpha_{C} \mathcal{F}_{C}\left(\varepsilon_{e q}\right) \\
\mathcal{G}\left(\varepsilon_{i}\right)=\mathcal{G}_{T}\left(\varepsilon_{i}\right) D_{T}+\mathcal{G}_{C}\left(\varepsilon_{i}\right) D_{C}
\end{gathered}
$$


em que:

$$
\begin{gathered}
\mathcal{F}_{T}\left(\varepsilon_{e q}\right)=\frac{\varepsilon_{d 0}\left(1-A_{T}\right)}{\varepsilon_{e q}^{2}}+\frac{A_{T} B_{T}}{e^{B_{T}\left(\varepsilon_{e q}-\varepsilon_{d 0}\right)}} ; \quad \mathcal{F}_{C}\left(\varepsilon_{e q}\right)=\frac{\varepsilon_{d 0}\left(1-A_{C}\right)}{\varepsilon_{e q}^{2}}+\frac{A_{C} B_{C}}{e^{B_{C}\left(\varepsilon_{e q}-\varepsilon_{d 0}\right)}} \\
\mathcal{I}_{T}\left(\varepsilon_{i}\right)=\frac{\partial \alpha_{T}}{\partial \varepsilon_{i}} ; \quad \mathcal{I}_{C}\left(\varepsilon_{i}\right)=\frac{\partial \alpha_{C}}{\partial \varepsilon_{i}}
\end{gathered}
$$

Para o caso de solicitação radial, $\alpha_{T}$ e $\alpha_{C}$ permanecem constantes ao longo da história de carregamento e, por essa razão, $\mathcal{G}_{T}$ e $\mathcal{G}_{C}$ anulam-se, PEREGO (1989). A expressão (A.1) é, então, reduzida a:

$$
\dot{D}=\mathcal{F}\left(\varepsilon_{e q}\right) \frac{\partial \varepsilon_{e q}}{\partial \varepsilon_{i}} \frac{\partial \varepsilon_{i}}{\partial t}=\boldsymbol{H}^{*} \cdot \dot{\varepsilon}
$$

em que:

$$
\begin{gathered}
\frac{\partial \varepsilon_{e q}}{\partial \varepsilon_{i}}=\frac{\left\langle\varepsilon_{i}\right\rangle_{+}}{\varepsilon_{e q}} \\
\boldsymbol{H}^{*} \stackrel{\text { def }}{=} \mathcal{F}\left(\varepsilon_{e q}\right) \frac{\partial \varepsilon_{e q}}{\partial \varepsilon_{i}}
\end{gathered}
$$

Integrando-se em um intervalo de tempo $\Delta t$ a relação constitutiva em taxas (4.6) e, introduzindo-se (A.5), chega-se, então, à expressão incremental correspondente à formulação tangente:

$$
\Delta \boldsymbol{\sigma}=(1-D) \boldsymbol{C}_{0} \Delta \boldsymbol{\varepsilon}-\left[\mathcal{F}\left(\varepsilon_{e q}\right) \frac{\partial \varepsilon_{e q}}{\partial \varepsilon_{i}} \Delta \varepsilon_{i}\right] \boldsymbol{C}_{0} \boldsymbol{\varepsilon}
$$

Para sua implementação, é interessante que a expressão acima seja reescrita em função das deformações definidas com relação aos eixos cartesianos e que, no segundo termo, a parte escalar seja colocada após a operação tensorial:

$$
\Delta \boldsymbol{\sigma}=(1-D) \boldsymbol{C}_{0} \Delta \boldsymbol{\varepsilon}-\boldsymbol{C}_{0} \varepsilon \mathcal{F}\left(\varepsilon_{e q}\right) \frac{\partial \varepsilon_{e q}}{\partial \boldsymbol{\varepsilon}} \Delta \boldsymbol{\varepsilon}
$$

onde, para evitar que a notação indicial misture-se com a tensorial definiu-se:

$$
\frac{\partial \varepsilon_{e q}}{\partial \varepsilon}=\frac{\partial \varepsilon_{e q}}{\partial \varepsilon_{i}} \frac{\partial \varepsilon_{i}}{\partial \varepsilon}
$$

Considera-se, agora, uma aproximação $\tilde{\boldsymbol{u}}(\boldsymbol{x})=\boldsymbol{\Phi}^{T} \boldsymbol{U}$ para o campo dos deslocamentos, obtida a partir da matriz das funções aproximadoras $\Phi$ e parâmetros genera- 
lizados $\boldsymbol{U}$. A forma aproximada para a expressão incremental da relação constitutiva pode ser, então, expressa como ${ }^{1}$ :

$$
\Delta \boldsymbol{\sigma}=[(1-D) \boldsymbol{C}_{0}-\boldsymbol{C}_{0} \overbrace{\boldsymbol{\varepsilon} \mathcal{F}\left(\varepsilon_{e q}\right) \frac{\partial \varepsilon_{e q}}{\partial \boldsymbol{\varepsilon}}}^{\boldsymbol{\varepsilon} \otimes} \boldsymbol{H}^{*} \quad \boldsymbol{B} \Delta \boldsymbol{u}
$$

onde $\boldsymbol{B}=\boldsymbol{L} \boldsymbol{\Phi}^{T}$ e $\boldsymbol{L}$ é o operador que relaciona os campos de deslocamento e deformação e $(\boldsymbol{a} \otimes \boldsymbol{b})$ é a notação adotada para indicar o produto diádico ou tensorial entre dois vetores $\boldsymbol{a}$ e $\boldsymbol{b}$ quaisquer.

A forma final da matriz de rigidez, obtida a partir da aproximação de Galerkin para o problema de evolução do dano, pode ser representada como:

$$
\boldsymbol{K}_{\mathrm{tg}}=\int_{\Omega} \boldsymbol{B}^{T}\left[(1-D) \boldsymbol{C}_{0}-\boldsymbol{C}_{0} \varepsilon \mathcal{F}\left(\varepsilon_{e q}\right) \frac{\partial \varepsilon_{e q}}{\partial \boldsymbol{\varepsilon}}\right] \boldsymbol{B} d \Omega
$$

Na expressão acima, por ser não-simétrica o termo entre colchetes, a matriz de rigidez perde sua simetria. Quando se adota a formulação secante, a expressão para matriz de rigidez permanece simétrica e se reduz a:

$$
\boldsymbol{K}_{\mathrm{sec}}=\int_{\Omega} \boldsymbol{B}^{T}\left[(1-D) \boldsymbol{C}_{0}\right] \boldsymbol{B} d \Omega
$$

\footnotetext{
${ }^{1}$ Observe que a abordagem dada é geral, sendo válida para qualquer um dos métodos de aproximação discutidos neste trabalho, MEF, MEFG ou Métodos Sem Malha.
} 


\section{Apêndice B}

\section{Partição da Unidade}

Uma da mais importantes características das funções de aproximação obtidas a partir da técnica dos MQM, (2.7), encontra-se no fato de constituirem uma partição da unidade. Segundo ODEN; REDDY (1976), em um domínio $\Omega$ em $\mathbb{R}^{\eta}$, com envoltória formada pela união de conjuntos abertos $\left\{G_{j}\right\}_{j=1}^{N}$, uma classe de funções $\psi_{j}(\boldsymbol{x})$ forma uma partição da unidade caso apresente as seguintes propriedades:

1. $\psi_{j}(\boldsymbol{x}) \in C_{0}^{\infty}\left(G_{j}\right)$;

2. $\sum_{j=1}^{N} \psi_{j}(\boldsymbol{x})=1$;

3. $\psi_{j}(\boldsymbol{x}) \geq 0$ em $\Omega$;

4. Todo sub-conjunto compacto de $\Omega$ intersepta apenas um número finito de suportes de $\psi_{j}(\boldsymbol{x})$.

Estas propriedades são bastante restritivas sendo, por isso, relaxadas nas interpretações realizadas para o Método das Nuvens $h p$, o MEFG e o MEF. Considera-se, por exemplo, as funções de forma Lagrangianas em $\mathbb{R}^{1}$, comumente empregadas no MEF (e também no MEFG), que são tomadas como exemplos de partição de unidade, embora as propriedades acima não sejam verificadas em sua totalidade. No caso das funções Lagrangianas Lineares, verifica-se apenas a continuidade $C_{0}^{0}\left(\omega_{j}\right)$ pois, sua primeira derivada, constante nos elementos pertencentes à nuvem $\omega_{j}$, além de ser contínua na face desses elementos já não mais apresenta um suporte compacto. As funções Lagrangianas de ordem superior $k$, por sua vez, podem assumir valores negativos em determinadas regiões, contrariando a terceira propriedade. Situação semelhante ocorre para as funções de aproximação obtidas a partir dos MQM de ordem $C_{0}^{\min (k, l)}$ 
como mostrado na seção 2.1, e observado em MENDONÇA; BARCELLOS; DUARTE (2000); ainda assim são consideradas partições de unidade por verificarem a propriedade 2, em particular. A propriedade mais importantes é a segunda sendo, por isso, comentada a seguir.

A segunda propriedade é essencial para se garantir a convergência da aproximação à medida que novos pontos sejam acrescentados ao domínio. Para entender essa afirmação considera-se a seguinte aproximação:

$$
\tilde{u}(\boldsymbol{x})=\sum_{j=1}^{N} \phi_{j} u_{j}
$$

em que $N$ corresponde ao conjunto de pontos nodais onde são definidos os parâmetros $u_{j}$. Caso a função a ser aproximada seja uma constante, $u(\boldsymbol{x})=k$, tem-se $u_{j}=$ $k, \forall j=1,2, \cdots, N$, fazendo-se com que:

$$
u(\boldsymbol{x}) \approx \tilde{u}(\boldsymbol{x})=\sum_{j=1}^{N} \phi_{j} k=k \sum_{j=1}^{N} \phi_{j}
$$

Caso o conjunto $\left\{\phi_{j}\right\}_{j=1}^{N}$ forme uma PU, obtém-se, empregando-se a propriedade 2:

$$
u(\boldsymbol{x}) \approx \tilde{u}(\boldsymbol{x})=k
$$

A verificação da propriedade 2 permite, portanto, que a aproximação $\tilde{u}(\boldsymbol{x})$ represente exatamente qualquer constante. Isto implica, no caso de deslocamentos, na capacidade de reproduzir movimentos de corpo rígido de translação, propriedade essencial para a convergência da solução:

$$
\lim _{N \rightarrow \infty} \tilde{u}(\boldsymbol{x})=u(\boldsymbol{x})
$$




\section{Apêndice C}

\section{Solução do Sistema de Equações no MEFG}

Seja o seguinte sistema de equações, obtido da aproximação de Galerkin de um PVC para a formulação do MEFG:

$$
\boldsymbol{K} \boldsymbol{U}=\boldsymbol{F}
$$

onde $\boldsymbol{K}$ é uma matriz semi-definida positiva. No caso do MEF convencional, para problemas de elasticidade por exemplo, a matriz de rigidez apresenta auto-valores associados às auto-funções que descrevem os deslocamentos de corpo rígido. Estes são removidos aplicando-se as condições de contorno. No MEFG, devido à dependência linear do conjunto de funções aproximadoras, surgem novas auto-funções cuja natureza é desconhecida. Desse modo, não há como eliminá-las previamente e, por isso, não se define a inversa $\boldsymbol{K}^{-1}$. Ainda assim, a solução do sistema (C.1) existe, apesar de não ser única. Para resolvê-lo, uma das alternativas propostas no trabalho de STROUBOULIS; BABUŠKA; COPPS (2000) consiste em se utilizar da estratégia de solução de MELENK (1992) em que se produz uma pequena pertubação na matriz de rigidez e, através de um procedimento iterativo, corrige-se a solução aproximada obtida para o novo sistema de equações. Esta estratégia, descrita a seguir, é neste trabalho referida como procedimento de Babuška.

Considera-se, então, $\boldsymbol{I}$ a matriz identidade e $\epsilon>0$ uma constante. A partir de $\boldsymbol{K}$ determina-se uma nova matriz, agora definida positiva e dada por:

$$
\boldsymbol{K}_{\epsilon}=\boldsymbol{K}+\epsilon \boldsymbol{I}
$$


Com a nova matriz $\boldsymbol{K}_{\epsilon}$ gera-se uma primeira aproximação (iteração 0) para a solução do sistema (C.1):

$$
\boldsymbol{U}_{0}=\boldsymbol{K}_{\epsilon}^{-1} \boldsymbol{F}
$$

Como $\boldsymbol{U}_{0}$ é aproximada, haverá um resíduo definido por:

$$
\boldsymbol{r}_{0}=\boldsymbol{F}-\boldsymbol{K} \boldsymbol{U}_{0}
$$

A aproximação $e_{0}$ para o primeiro erro do procedimento é definida como:

$$
\boldsymbol{e}_{0}=\boldsymbol{K}_{\epsilon}^{-1}\left(\boldsymbol{F}-\boldsymbol{K} \boldsymbol{U}_{0}\right)
$$

que de (C.4)

$$
\boldsymbol{e}_{0}=\boldsymbol{K}_{\epsilon}^{-1} \boldsymbol{r}_{0}
$$

A solução aproximada é, então, atualizada através de:

$$
\boldsymbol{U}_{1}=\boldsymbol{U}_{0}+\boldsymbol{e}_{0}
$$

cujo resíduo é:

$$
\boldsymbol{r}_{1}=\boldsymbol{F}-\boldsymbol{K} \boldsymbol{U}_{1}=\boldsymbol{F}-\boldsymbol{K}\left(\boldsymbol{U}_{0}+\boldsymbol{e}_{0}\right)
$$

que pode ser reformulado como:

$$
\boldsymbol{r}_{1}=\boldsymbol{r}_{0}-\boldsymbol{K} \boldsymbol{e}_{0}
$$

Repetindo-se (C.6), tem-se, para a iteração 1:

$$
\boldsymbol{e}_{1}=\boldsymbol{K}_{\epsilon}^{-1}\left(\boldsymbol{r}_{1}\right)
$$

Uma nova solução aproximada é, portanto, obtida:

$$
\boldsymbol{U}_{2}=\boldsymbol{U}_{1}+\boldsymbol{e}_{1}=\boldsymbol{U}_{0}+\left(\boldsymbol{e}_{0}+\boldsymbol{e}_{1}\right)
$$

e o resíduo correspondente é:

$$
\boldsymbol{r}_{2}=\boldsymbol{F}-\boldsymbol{K} \boldsymbol{U}_{2}=\boldsymbol{F}-\boldsymbol{K}\left(\boldsymbol{U}_{1}+\boldsymbol{e}_{1}\right)=\boldsymbol{F}-\boldsymbol{K}\left(\boldsymbol{U}_{0}+\boldsymbol{e}_{0}+\boldsymbol{e}_{1}\right)
$$


que reformulado determina:

$$
\boldsymbol{r}_{2}=\boldsymbol{r}_{0}-\boldsymbol{K}\left(\boldsymbol{e}_{0}+\boldsymbol{e}_{1}\right)
$$

O erro aproximado para a iteração 2 é, então, dado por:

$$
\boldsymbol{e}_{2}=\boldsymbol{K}_{\epsilon}^{-1} \boldsymbol{r}_{2}
$$

e a próxima solução aproximada é:

$$
\boldsymbol{U}_{3}=\boldsymbol{U}_{2}+\boldsymbol{e}_{2}=\boldsymbol{U}_{0}+\left(\boldsymbol{e}_{0}+\boldsymbol{e}_{1}+\boldsymbol{e}_{2}\right)
$$

As expressões (C.13,C.14,C.15) devem ser repetidas até que uma determinada medida de erro seja pequena o suficiente. No algoritmo C.1 representa-se esse procedimento de solução.

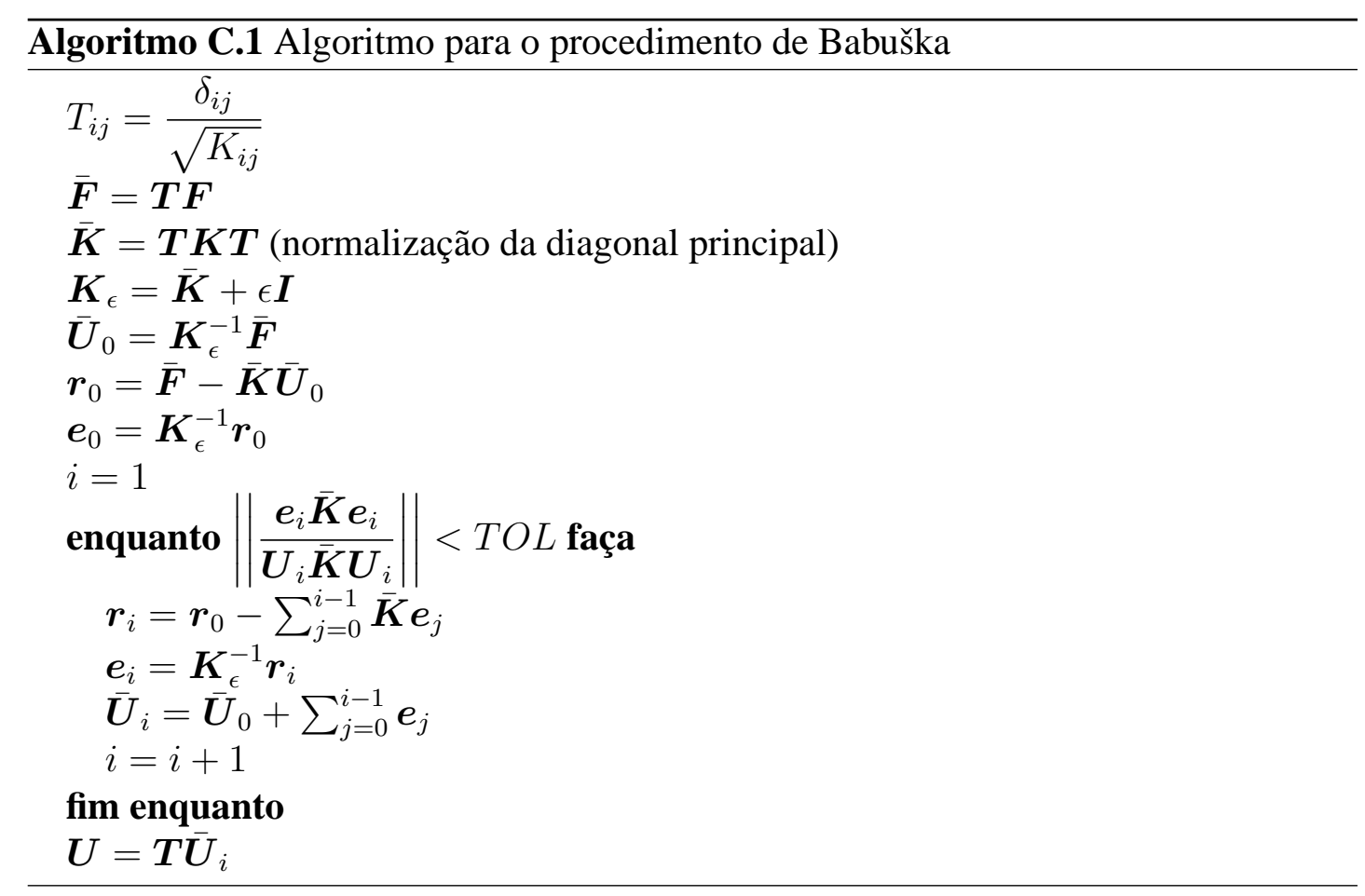

Em DUARTE; BABUŠKA; ODEN (2000) sugere-se que a matriz de rigidez tenha sua diagonal normalizada, gerando-se um sistema de equações equivalente $\overline{\boldsymbol{K}} \overline{\boldsymbol{U}}=$ $\overline{\boldsymbol{F}}$. Esta estratégia visa reduzir os erros durante o processo iterativo, fazendo-se com que a pertubação na diagonal principal seja independente da ordem de grandeza dos elementos de $\boldsymbol{K}$. 
Em STROUBOULIS; BABUŠKA; COPPS (2000), adota-se $\epsilon \approx 10^{-10}$, o que faz, segundo os autores, com que em uma única iteração e trabalhando-se em precisão dupla, já se tenha a convergência do método de solução.

Como alternativa ao procedimento iterativo, existe, ainda, o método direto de eliminação de Gauss multifrontal para sistemas simétricos indefinidos proposto em DUFF; REID (1983) e cuja implementação é disponibilizada na Harwell Subroutines Library, HSL (2000), através do pacote MA27 em linguagem FORTRAN77. Em STROUBOULIS; BABUŠKA; COPPS (2000) afirma-se que o emprego do método multifrontal é mais vantajoso pois se ganha em velocidade na solução do sistema de equações. 


\section{Apêndice D}

\section{Espaço de Aproximação Polinomial}

Em $\mathbb{R}^{2}$, as funções de forma do MEF para elementos quadriláteros, construídas através de interporlações Serendípetas, BATHE (1996), ou em formulação hierárquica, SZABÓ; BABUŠKA (1991), representam exatamente espaços polinomiais $\mathcal{P}_{p}$. Tais espaços, são gerados, de acordo com o esquema da Figura D.1, pelos monômios:

$$
x^{i} y^{i} \text { sendo }\left\{\begin{array}{c}
i, j=0,1, \cdots, p \\
i+j=0,1, \cdots, p \\
x y \text { para } p=1 \\
x^{p} y, x y^{p} \text { para } p \neq 1
\end{array}\right.
$$

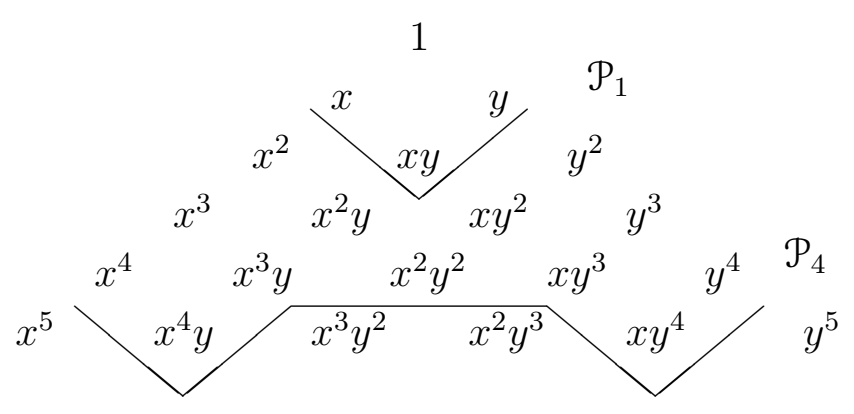

Figura D.1: Monômios representados 


\section{Apêndice E}

\section{Discussão Sobre o Erro A Priori}

Para uma envoltória de $\bar{\Omega}$ formada pelas nuvens $\left\{\omega_{j}\right\}_{j=1}^{N}$ associadas às funções de PU $\left\{\phi_{j}\right\}_{j=1}^{N}$, são impostas as seguintes condições em DUARTE (1996):

1. para toda posição do domínio $\bar{\Omega}$ existe um número máximo de nuvens $\rho \in \mathbb{N}$, independente de $N$, sobrepostas sobre essa posição;

2. $\left\|\phi_{j}\right\|_{L_{\infty}(\Omega)} \leq C_{\infty}$

3. $\left\|\nabla \phi_{j}\right\|_{L_{\infty}(\Omega)} \leq \frac{C_{G}}{h_{j}}$

onde $C_{\infty}$ e $C_{G}$ são duas constantes e $h_{j}$ representa a dimensão característica da nuvem e $\|\boldsymbol{u}\|_{L_{\infty}(\Omega)}=\max |\boldsymbol{u}|$ é a norma uniforme ou de Chebyshev, RIVLIN (1969)

Com base nestas condições, demonstra-se que para uma família de Nuvens- $h p$, $\Im_{N}^{k=1, p}$ (onde se incluem as funções do MEFG) o erro estimado a priori de uma aproximação obtida para a solução $u \in \mathcal{H}^{r}, r \geq 1$ é tal que:

$$
\begin{gathered}
|\boldsymbol{u}-\tilde{\boldsymbol{u}}|_{\mathcal{H}^{1}(\Omega)} \leq \sqrt{2} \rho C\left(C_{G}^{2}+C_{\infty}^{2}\right)^{\frac{1}{2}} h^{p}|\boldsymbol{u}|_{\mathcal{H}^{p+1}(\Omega)} \quad \text { para a versão } h \\
|\boldsymbol{u}-\tilde{\boldsymbol{u}}|_{\mathcal{H}^{1}(\Omega)} \leq \sqrt{2} \rho C\left(\frac{C_{G}^{2}}{h_{\text {min }}^{2}}+C_{\infty}^{2}\right)^{\frac{1}{2}} e^{-\gamma p}|\boldsymbol{u}|_{\mathcal{H}^{r}(\Omega)} \quad \text { para a versão } p
\end{gathered}
$$

onde:

- $C$ e $\gamma$ são constantes que dependem unicamente de $\Omega$ e $\bar{\Omega}$;

- $h_{\min }=\min _{j=1, \cdots, N} h_{j}$

- $h=\max _{j=1, \cdots, N} h_{j}$; 
- $p=\min _{j=1, \cdots, N} p_{j}$

- $p_{j}$ é o grau máximo do polinômio completo representado pelas funções da nuvem $\omega_{j} ;$

- $|u|_{\mathcal{H}^{m}(\Omega)}=\left(\sum_{|\alpha|=m}\left\|D^{\alpha} \boldsymbol{u}\right\|_{L_{2}(\Omega)}^{2}\right)^{1 / 2}$ é a semi-norma no espaço $\mathcal{H}^{m}$, segundo ODEN; REDDY (1976);

- $D^{\alpha} \boldsymbol{u}(\boldsymbol{x})=\frac{\partial^{|\alpha|} \boldsymbol{u}(\boldsymbol{x})}{\partial x_{1}^{\alpha_{1}} \cdots \partial x_{n}^{\alpha_{n}}}$ corresponde ao operador de derivadas múltiplas em $\mathbb{R}^{\eta}, \boldsymbol{x}=\left(x_{1}, \cdots, x_{\eta}\right)$, na ordem $|\alpha|=\alpha_{1}+\cdots+\alpha_{\eta} ;$

- $\|\boldsymbol{u}\|_{L_{p}(\Omega)}=\left(\int_{\Omega} \boldsymbol{u}^{p} d \Omega\right)^{1 / p}$ é a norma em $L_{p} ;$

A interpretação destas estimativas de erro pode ser dada para o MEFG como se segue. Considera-se, uma nuvem $\omega_{j}$ de elementos caracterizada pela dimensão $h_{j}$. Variando-se a posição do nó $\boldsymbol{x}_{j}$, sem que $h_{j}$ seja alterado, de maneira que esse se aproxime de algum dos nós vizinhos é de se esperar que o gradiente da função $\phi_{j}$, próximo a $\boldsymbol{x}_{j}$ cresça bastante, caracterizando a distorção dos elementos dessa nuvem. Como $h_{j}$ mantém-se constante, pela condição (3) $C_{G}$ deve apresentar um valor maior e, como conseqüência, os limites superiores para o erro na semi-norma $\mathcal{H}^{1}$ em (E.1) e (E.2) são elevados. Ainda que o erro de aproximação aumente, nenhuma alteração deve ocorrer nas taxas de convergência em $\mathcal{H}^{1}$, que permanecem sendo definidas pelos parâmetros $p$ e $-\gamma p$. Em problemas de elasticidade, pode-se mostrar a equivalência entre a semi-norma $|\boldsymbol{u}|_{\mathcal{H}^{1}}$ e a norma energia $\|\boldsymbol{u}\|_{u}=\sqrt{(\mathcal{B}(u, u))}$ que, por sua vez, é derivada da energia de deformação. Explica-se, assim, a discussão ao final da seção 3.2.1, na qual se observa que a distorção da malha introduz um erro na aproximação do MEFG sem, contudo, prejudicar a sua taxa de convergência com relação à energia de deformação. 


\section{Apêndice F}

\section{Detalhes da Implementação do MEFG}

\section{F.1 Convergência no Método de Newton-Raphson}

Em análises feitas através do MEF, é comum utilizar-se, no critério de parada do processo iterativo de Newton-Raphson, algumas das seguintes medidas:

- Norma $L_{2}$ dos incrementos de deslocamento:

$$
\left\|\Delta \boldsymbol{U}^{\left(i_{t}\right)}\right\|_{2} \stackrel{\text { def }}{=} \sqrt{\sum_{i=1}^{N G L}\left(\Delta U_{i}^{\left(i_{t}\right)}\right)^{2}}
$$

- Norma $L_{2}$ do desequilíbrio em forças:

$$
\left\|\boldsymbol{\psi}^{\left(i_{t}\right)}\right\|_{2} \stackrel{\text { def }}{=} \sqrt{\sum_{i=1}^{N G L}\left(\psi_{i}^{\left(i_{t}\right)}\right)^{2}}
$$

- Norma de energia incremental:

$$
\left\|\Delta \boldsymbol{u}_{p}\right\|_{u} \stackrel{\text { def }}{=} \sqrt{\Delta \boldsymbol{U}^{\left(i_{t}\right)^{T}} \boldsymbol{\psi}^{\left(i_{t}-1\right)}}=\sqrt{\sum_{i=2}^{N G L}\left(\Delta U_{i}^{\left(i_{t}\right)} \psi_{i}^{\left(i_{t}-1\right)}\right)}
$$

No MEFG, contudo, o ideal é que se utilize, no critério de parada para as iterações, apenas a norma de energia. A razão para isso encontra-se na maneira com que se realiza o enriquecimento da PU. Considera-se, então, a expressão (2.36) da aproxima- 
ção no MEFG:

$$
u_{p}(\boldsymbol{x})=\sum_{j=1}^{N} \mathcal{N}_{j}(\boldsymbol{x})\left\{u_{j}+\sum_{i=1}^{q_{j}(p)} p_{j i}(\boldsymbol{x}) b_{j i}\right\}
$$

Uma maneira de interpretar a expressão acima é considerando-se $\tilde{u}(\boldsymbol{x})$ como uma combinação linear das funções de $\Im_{N}^{k=1, p}=\left\{\left\{\mathcal{N}_{j}(\boldsymbol{x})\right\}_{j=1}^{N} \cup\left\{\mathcal{N}_{j}(\boldsymbol{x}) p_{j i}(\boldsymbol{x})\right\}_{j=1}^{N} \mid i \in \mathcal{J}_{j}\right\}$ através dos parâmetros $\left\{\left\{u_{j}, b_{j i}\right\}_{j=1}^{N} \mid i \in \mathcal{J}_{j}\right\}$. De acordo com a seção 2.5.1, a família de funções $\Im_{N}^{k=1, p}$, é formada geralmente por funções linearmente dependentes. Sendo assim, essa combinação pode admitir um conjunto infinito de parâmetros. Para ser mais exato, tal afirmação é válida apenas para os parâmetros $b_{i j}$, enquanto que os $u_{j}$ são unicamente definidos ${ }^{1}$. Por exemplo, seja uma situação em que $u_{p}(\boldsymbol{x})=0$. Uma das soluções possíveis seria, naturalmente, $\left\{\{0\}_{j=1}^{N} \mid i \in \mathcal{J}_{j}\right\}$; ela, porém, não é única e outras soluções do tipo $\boldsymbol{U}^{T}=\left\{\left\{u_{j}=0, b_{j i} \neq 0\right\}_{j=1}^{N} \mid i \in \mathcal{J}_{j}\right\}$ também são admitidas.

Durante o processo de solução do problema não-linear, seção 5.3, calcula-se para cada iteração $i_{t}$ do tempo $t$ a seguinte função, obtida de acréscimos dos parâmetros de (F.4):

$$
\Delta u_{p}^{\left(i_{t}\right)}(\boldsymbol{x})=\sum_{j=1}^{N} \mathcal{N}_{j}(\boldsymbol{x})\left\{\Delta u_{j}^{\left(i_{t}\right)}+\sum_{i=1}^{q_{j}(p)} p_{j i}(\boldsymbol{x}) \Delta b_{j i}^{\left(i_{t}\right)}\right\}
$$

que é empregada para atualizar a solução total até o passo $t$ :

$$
{ }^{t} u_{p}^{\left(i_{t}\right)}(\boldsymbol{x})={ }^{t} u_{p}{ }^{\left(i_{t}-1\right)}(\boldsymbol{x})+\Delta u_{p}^{\left(i_{t}\right)}(\boldsymbol{x})
$$

De forma análoga ao discutido anteriormente, $\Delta \tilde{u}^{\left(i_{t}\right)}(\boldsymbol{x})=0$ não implica necessariamente em se ter $\left\{\left\{\Delta u_{j}^{i_{t}}=\mathbf{0}, \Delta b_{j i}^{i_{t}}=\mathbf{0}\right\}_{j=1}^{N} \mid i \in \mathcal{J}_{j}\right\}$; como conseqüência, a norma dos incrementos de deslocamento (F.1) pode ser diferente de zero, apesar dos resultados terem convergido. Um raciocínio equivalente pode ser conduzido para que a mesma conclusão seja obtida para o vetor dos resíduos $\psi^{\left(i_{t}-1\right)}$. Na abordagem do MEFG, o emprego das normas de incrementos de deslocamento, (F.1), ou do desequilíbrio em forças, (F.2), não é, portanto, adequado para o controle de convergência da solução iterativa do método de Newton-Raphson. Resta, então, a norma de energia incremental, (F.3), obtida pelo produto escalar dos vetores $\Delta \boldsymbol{U}^{i_{t} T}$ e $\boldsymbol{\psi}^{\left(i_{t}-1\right)}$. Esta norma, independentemente das funções aproximadoras empregadas, está relacionada ao trabalho realizado pelo desequilíbrio de forças sobre os deslocamentos incrementais e deve

\footnotetext{
${ }^{1}$ É essa a razão de se utilizar o procedimento de Babuška descrito no apêndice C. Procura-se, através da perturbação da diagonal principal, criar uma situação favorável para se encontrar um dos conjuntos possíveis de solução para $b_{j i}$.
} 
se anular quando a solução convergir, ou seja, quando se verificar o equilíbrio entre as forças externas e internas.

\section{F.2 Consideração da armadura}

Em problemas semelhantes ao da viga de concreto armado da seção 5.1, quando analisados pelo MEF no domínio $\mathbb{R}^{2}$, é usual considerar a presença de dois materiais diferentes, o aço e o concreto. Para cada elemento finito deve-se, então, definir, além da incidência nodal, o material correspondente. A altura dos elementos associados ao material aço é obtida dividindo-se a área total de cada camada de armadura pela espessura da seção transversal. Como em geral a área é bem pequena, o elemento de aço resulta bastante fino em relação às dimensões da estrutura considerada. Este fator impõe uma restrição às análises com o MEF hierárquico e, especialmente com o MEFG, em que se procura empregar um número reduzido de elementos relativamente grandes, priorizando-se o enriquecimento polinomial da aproximação.

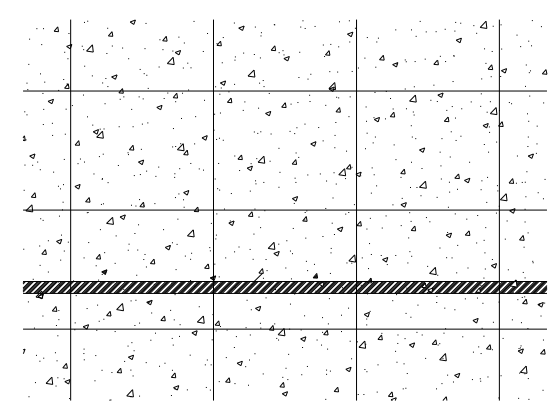

Figura F.1: Aço embutido nos elementos finitos com o material concreto

Para contornar este problema, RANJBARAN (1991) considera a armadura embutida no elemento finito, como é mostrado na Figura F.1. A validade dessa estratégia está vinculada à hipótese de perfeita aderência entre o concreto e o aço. Garante-se, assim, que exista uma continuidade no tensor de deformações na fronteira entre os dois materiais. O meio pode, dessa forma, ser modelado como um único material cuja a rigidez é função das contribuições do aço e concreto. Apenas a rigidez axial da barra de aço é considerada, sendo distribuída, nos elementos em que estiver presente de acordo com sua posição com relação aos pontos nodais. Detalhes sobre a formulação matemática e implementação dessa estratégia podem ser encontrados, além de RANJBARAN (1991), em ASSAN (1999). 


\section{Apêndice G}

\section{Interpretação do Estimador do MRE}

Em ODEN (1989), procura-se aproximar a função de erro exato por uma função do erro entre $\boldsymbol{u}_{p}$ e uma solução mais enriquecida, representada genericamente por $\boldsymbol{u}_{p+1} \in X_{p+1}$, ou seja:

$$
\boldsymbol{e}_{p} \stackrel{\text { substituído }}{\longrightarrow} \boldsymbol{u}_{p+1}-\boldsymbol{u}_{p}
$$

A justificativa está no que segue. Sendo $\boldsymbol{e}_{p+1}=\boldsymbol{u}-\boldsymbol{u}_{p+1}$ a função de erro para a aproximação $\boldsymbol{u}_{p+1}$ e com base na condição de ortogonalidade (6.3), pode-se mostrar que:

$$
\left\|\boldsymbol{e}_{p}\right\|_{\mathfrak{U}}^{2}=\left\|\boldsymbol{u}_{p+1}-\boldsymbol{u}_{p}\right\|_{\mathfrak{U}}^{2}+\left\|\boldsymbol{e}_{p+1}\right\|_{\mathfrak{U}}^{2}
$$

Dessa forma, se o espaço $X_{p+1}$, no qual é definida $\boldsymbol{u}_{p+1}$ for de dimensão muito maior do que o $x_{p}$ torna-se justificável fazer a seguinte estimativa:

$$
\left\|\boldsymbol{e}_{p}\right\|_{u} \approx\left\|\boldsymbol{u}_{p+1}-\boldsymbol{u}_{p}\right\|_{u}
$$

A função de erro procurada passa a ser, dessa maneira, $\boldsymbol{u}_{p+1}-\boldsymbol{u}_{p}$. Uma maneira bastante simples de se interpretar tal aproximação para o erro pode ser encontrada em ZIENKIEWICZ; MORGAN (1983) ao se fazer uma analogia com a expansão de Taylor. Afirma-se que, no limite, quando o tamanho $h$ dos elementos finitos tender a zero (de maneira análoga, quando a dimensão característica $h$ das nuvens tender a zero), o erro passa a ser de um grau superior ao da função de aproximação empregada. Com base nessa argumentação, justifica-se a descrição do erro em um espaço de funções que representem polinômios de um grau superior ao do espaço da aproximação obtida para 
a solução. Além disso, como no limite, espera-se que a parcela mais importante do erro seja de um grau superior ao da função de aproximação, não há porque se utilizar as funções presentes no espaço da solução aproximada para se descrever o erro.

Este argumento torna natural a escolha das funções bolha descritas na seção 6.3, pois no espaço $X_{p+1}^{0}(\mathcal{K})$ não estão presentes as funções do espaço $X_{p}$ da solução $\boldsymbol{u}_{p}$. Como consequiência, o custo computacional para a solução do problema (6.33) é reduzido significativamente. Outro argumento válido para se utilizar $X_{p+1}^{0}$ é discutido no início da seção 6.4, como uma maneira de garantir a eliminiação de modos equivalentes aos movimentos de corpo rígido.

Além desse fato, em ODEN (1989), demonstra-se que, se a função $\boldsymbol{u}_{p+1}-\boldsymbol{u}_{p}$ é aproximada pelas funções de $X_{p+1}^{0}$, garante-se que o estimador $\left\|\tilde{\boldsymbol{e}}_{p}\right\|_{u}$, obtido de (6.46), apresente o mesmo comportamento que a norma energia do erro exato, ou seja:

$$
C_{1}\left\|\tilde{\boldsymbol{e}}_{p}\right\|_{u} \leq\left\|\boldsymbol{e}_{p}\right\|_{u} \leq C_{2}\left\|\tilde{\boldsymbol{e}}_{p}\right\|_{u}
$$

onde $C_{1}$ e $C_{2}$ são constantes positivas. Condição, essa, essencial para que se tenha uma boa estimativa do erro.

A medida $\left\|\tilde{\boldsymbol{e}}_{p}\right\|_{u}$ é, portanto, uma estimativa da aproximação de Galerkin no problema (6.33) da função $\boldsymbol{u}_{p+1}-\boldsymbol{u}_{p}$ e, em vista da propriedade (G.4), pode ser usado como estimador, na norma de energia, para o erro $\boldsymbol{e}_{p}$ definido em (6.19). 


\section{Apêndice H}

\section{Equilíbrio do Vetor de Forças Generalizadas}

Seja a parcela relativa ao elemento $\mathcal{K}$ do vetor de forças nodais definido em (3.25), para os dados $(\boldsymbol{b}, \hat{t})$ :

$$
\begin{aligned}
{ }^{x} \boldsymbol{F}_{j}^{\mathcal{K}} & =\iint_{\mathcal{K}} \phi_{j} b_{x} l_{z} d x d y+\int_{\partial \mathcal{K} \cap \Gamma_{N}} \phi_{j} \hat{t}_{x} l_{z} d s \\
{ }^{y} \boldsymbol{F}_{j}^{\mathcal{K}} & =\iint_{\mathcal{K}} \boldsymbol{\phi}_{j} b_{y} l_{z} d x d y+\int_{\partial \mathcal{K} \cap \Gamma_{N}} \boldsymbol{\phi}_{j} \hat{t}_{y} l_{z} d s
\end{aligned}
$$

em que $j$ refere-se ao nó $\boldsymbol{x}_{j}$ do elemento $\mathcal{K}$ no qual os vetores de forças nodais equivalentes nas direções $x,{ }^{x} \boldsymbol{F}_{j}$, e $y,{ }^{y} \boldsymbol{F}_{j}$, são associados. Observa-se que a representação adotada, com a caracterização das componentes nas direções $x$ e $y$ diverge um pouco do restante do texto. Esta mudança é proposital e tem a finalidade de conferir mais clareza às expressões que seguem. Dessa forma, $\phi_{j}$, diferentemente de (3.18), está em letra minúscula e é representada pelo seguinte vetor:

$$
\phi_{j}^{T}=\{\mathcal{N}_{j} \overbrace{\overbrace{L_{j 1} \mathcal{N}_{j}} \cdots}^{\boldsymbol{\psi}_{j}} \overbrace{L_{j p_{j}} \mathcal{N}_{j}}\}
$$

onde $\boldsymbol{\psi}_{j}$ é o vetor das funções resultantes do enriquecimento de $\mathcal{N}_{j}$.

Somando-se, então, os vetores de (H.1) para todos os nós do elemento $\mathcal{K}$ : 


$$
\begin{aligned}
\sum_{j \in \mathcal{K}}{ }^{x} \boldsymbol{F}_{j}^{\mathcal{K}} & =\iint_{\mathcal{K}}\left(\sum_{j \in \mathcal{K}} \boldsymbol{\phi}_{j}\right) b_{x} l_{z} d x d y+\int_{\partial \mathcal{K} \cap \Gamma_{N}}\left(\sum_{i \in \mathcal{K}} \boldsymbol{\phi}_{j}\right) \hat{t}_{x} l_{z} d s \\
\sum_{j \in \mathcal{K}}{ }^{y} \boldsymbol{F}_{j}^{\mathcal{K}} & =\iint_{\mathcal{K}}\left(\sum_{j \in \mathcal{K}} \boldsymbol{\phi}_{j}\right) b_{y} l_{z} d x d y+\int_{\partial \mathcal{K} \cap \Gamma_{N}}\left(\sum_{j \in \mathcal{K}} \boldsymbol{\phi}_{j}\right) \hat{t}_{y} l_{z} d s
\end{aligned}
$$

Como $\left\{\mathcal{N}_{j}\right\}_{j \in \mathcal{K}}$ formam uma PU, sabe-se que $\sum_{j \in \mathcal{K}} \mathcal{N}_{j}=1$ e, por essa razão, as expressões (H.3) podem ser convenientemente reescritas como:

$$
\begin{aligned}
\sum_{j \in \mathcal{K}}{ }^{x} \boldsymbol{F}_{j}^{\mathcal{K}} & =\iint_{\mathcal{K}}\left[\begin{array}{c}
1 \\
\sum_{j \in \mathcal{K}} \boldsymbol{\psi}_{j}
\end{array}\right] b_{x} l_{z} d x d y+\int_{\partial \mathcal{K} \cap \Gamma_{N}}\left[\begin{array}{c}
1 \\
\sum_{j \in \mathcal{K}} \boldsymbol{\psi}_{j}
\end{array}\right] \hat{t}_{x} l_{z} d s \\
\sum_{j \in \mathcal{K}}{ }^{y} \boldsymbol{F}_{j}^{\mathcal{K}} & =\iint_{\mathcal{K}}\left[\begin{array}{c}
1 \\
\sum_{j \in \mathcal{K}} \boldsymbol{\psi}_{j}
\end{array}\right] b_{y} l_{z} d x d y+\int_{\partial \mathcal{K} \cap \Gamma_{N}}\left[\begin{array}{c}
1 \\
\sum_{j \in \mathcal{K}} \boldsymbol{\psi}_{j}
\end{array}\right] \hat{t}_{y} l_{z} d s
\end{aligned}
$$

ou seja:

$$
\begin{aligned}
\sum_{j \in \mathcal{K}}{ }^{x} \boldsymbol{F}_{j}^{\mathcal{K}}= & {\left[\iint_{\mathcal{K}}\left(\sum_{j \in \mathcal{K}} \boldsymbol{\psi}_{j}\right) b_{x} l_{z} d x d y+\int_{\partial \mathcal{K} \cap \Gamma_{N}}^{R_{x}^{\mathcal{K}}}\left(\sum_{i \in \mathcal{K}} \boldsymbol{\psi}_{j}\right) \hat{t}_{x} l_{z} d s\right] } \\
\sum_{j \in \mathcal{K}}{ }^{y} \boldsymbol{F}_{j}^{\mathcal{K}}= & {\left[\iint_{\mathcal{K}}\left(\sum_{j \in \mathcal{K}} \boldsymbol{\psi}_{j}\right) b_{y} l_{z} d x d y+\int_{\partial \mathcal{K} \cap \Gamma_{N}}\left(\sum_{i \in \mathcal{K}} \boldsymbol{\psi}_{j}\right) \hat{t}_{y} l_{z} d s\right] }
\end{aligned}
$$

onde, $\mathcal{R}_{x}^{\mathcal{K}}$ e $\mathcal{R}_{y}^{\mathcal{K}}$ são as resultantes de $(\boldsymbol{b}, \hat{t})$ que agem sobre o elemento $\mathcal{K}$, na direção $x$ e $y$, respectivamente:

$$
\begin{aligned}
& \mathcal{R}_{x}^{\mathcal{K}}=\iint_{\mathcal{K}} b_{x} l_{z} d x d y+\int_{\partial \mathcal{K} \cap \Gamma_{N}} \hat{t}_{x} l_{z} d s \\
& \mathcal{R}_{y}^{\mathcal{K}}=\iint_{\mathcal{K}} b_{y} l_{z} d x d y+\int_{\partial \mathcal{K} \cap \Gamma_{N}} \hat{t}_{y} l_{z} d s
\end{aligned}
$$

Considera-se, agora, os valores $\rho_{j}^{x}$ e $\rho_{j}^{y}$ que representam as distâncias em $x$ e em $y$ entre o nó $\boldsymbol{x}_{j}$ e uma posição qualquer $\left(x_{0}, y_{0}\right)$ do elemento $\mathcal{K}$. Pode-se, então, 
definir o seguinte vetor com os momentos nodais equivalentes associados ao nó $\boldsymbol{x}_{j}$ do elemento $\mathcal{K}$ :

$$
\begin{aligned}
\boldsymbol{M}_{j}^{\mathcal{K}} \stackrel{\text { def }}{=} \iint_{\mathcal{K}}\left[\begin{array}{c}
\sum_{j \in \mathcal{K}} \rho_{j}^{y} \mathcal{N}_{j} \\
\sum_{j \in \mathcal{K}} \rho_{j}^{y} \boldsymbol{\psi}_{j}
\end{array}\right] b_{x} l_{z} d x d y+\int_{\partial \mathcal{K} \cap \Gamma_{N}}\left[\begin{array}{c}
\sum_{j \in \mathcal{K}} \rho_{j}^{y} \mathcal{N}_{j} \\
\sum_{j \in \mathcal{K}} \rho_{j}^{y} \boldsymbol{\psi}_{j}
\end{array}\right] \hat{t}_{x} l_{z} d s \\
-\iint_{\mathcal{K}}\left[\begin{array}{c}
\sum_{j \in \mathcal{K}} \rho_{j}^{x} \mathcal{N}_{j} \\
\sum_{j \in \mathcal{K}} \rho_{j}^{x} \boldsymbol{\psi}_{j}
\end{array}\right] b_{y} l_{z} d x d y+\int_{\partial \mathcal{K} \cap \Gamma_{N}}\left[\begin{array}{c}
\sum_{j \in \mathcal{K}} \rho_{j}^{x} \mathcal{N}_{j} \\
\sum_{j \in \mathcal{K}} \rho_{j}^{x} \boldsymbol{\psi}_{j}
\end{array}\right] \hat{t}_{y} l_{z} d s
\end{aligned}
$$

em que se adota o sentido horário como positivo para o momento.

Para elementos em que o mapeamento entre as coordenadas naturais e as reais do elemento for linear, pode-se assumir que:

$$
\begin{aligned}
\rho^{x}(\boldsymbol{x}) & =\sum_{j \in \mathcal{K}} \rho_{j}^{x} \mathcal{N}_{j} \\
\rho^{y}(\boldsymbol{x}) & =\sum_{j \in \mathcal{K}} \rho_{j}^{y} \mathcal{N}_{j}
\end{aligned}
$$

Substituindo-se, então, as expressões (H.8) em (H.7) e realizando-se o somatório para todos os nós de $\mathcal{K}$ chega-se a:

$$
\sum_{j \in \mathcal{K}} \boldsymbol{M}_{j}^{\mathcal{K}}=\left[\begin{array}{c}
\mathcal{M}^{\mathcal{K}} \\
\left.\iint_{\mathcal{K}} \sum_{j \in \mathcal{K}}\left(\rho_{j}^{y} \boldsymbol{\psi}_{j} b_{x}+\rho_{j}^{x} \boldsymbol{\psi}_{j} b_{y}\right) l_{z} d x d y+\right] \\
\int_{\partial \mathcal{K} \cap \Gamma_{N}} \sum_{j \in \mathcal{K}}\left(\rho_{j}^{y} \boldsymbol{\psi}_{j} \hat{t}_{x}+\rho_{j}^{x} \boldsymbol{\psi}_{j} \hat{t}_{y}\right) l_{z} d s
\end{array}\right]
$$

onde:

$$
\mathcal{M}^{\mathcal{K}}=\iint_{\mathcal{K}} \rho^{y} b_{x}+\rho^{x} b_{y} l_{z} d x d y+\int_{\partial \mathcal{K} \cap \Gamma_{N}} \rho^{y} \hat{t}_{x}+\rho^{x} \hat{t}_{y} l_{z} d s
$$

correspondente ao momento das forças atuantes sobre o elemento $\mathcal{K}$.

De (H.5) e (H.9), observa-se que as componentes de $\boldsymbol{F}_{j}^{\mathcal{K}}$ e $\boldsymbol{M}_{j}^{\mathcal{K}}$ associadas à partição da unidade recuperam o equilíbrio em força e momento do carregamento atuante sobre um determinado elemento. As demais componentes, associadas ao enriquecimento são consideradas, portanto, como auto-equilibradas.

A demonstração foi realizada para o conjunto de dados $(\boldsymbol{b}, \hat{t})$ mas as mesmas conclusões podem ser obtidas, por analogia, para $\left(\boldsymbol{r}_{\Omega}, \boldsymbol{r}_{\Gamma},\left[\boldsymbol{t}\left(\boldsymbol{u}_{p}\right)\right], \boldsymbol{\theta}^{\mathcal{K}}\right)$ do MRE equilibrado discutido na seção 6.4.1. 


\section{Apêndice I}

\section{Transferência de Valores Associados aos Pontos de Gauss}

A estratégia sugerida a seguir, assemelha-se ao procedimento empregado para recuperar um campo de tensões "suavizado" em ZIENKIEWICZ; ZHU (1992a). Basicamente, parte-se do método dos mínimos quadrados para ajustar uma função a um conjunto de variáveis associadas aos pontos de Gauss. Este ajuste é realizado independentemente para cada nuvem, de modo que em um determinado elemento gerem-se tantas funções quanto o número de nuvens que o contêm. A partir daí, para se obter uma descrição única em todo o domínio, emprega-se a PU para "costurar" as diversas funções de cada nuvem.

Considera-se, portanto, uma nuvem $\omega_{j}$ que contenha um conjunto de elementos $\mathcal{K}$. Em cada elemento existem um número de $N G$ pontos de Gauss, $\left\{\boldsymbol{x}_{g}\right\}_{g=1}^{N G}$, nos quais encontram-se definidos um conjunto de valores $\left\{\varsigma_{g}\right\}_{g=1}^{N G}$ (tais valores representam, por exemplo, o dano $D$ ). Para essa nuvem, é possível definir uma função de ajuste, através da combinação linear entre os vários monômios da base $\boldsymbol{P}=\left\{p_{i}\right\}_{i=1}^{n},(n \leq m)$ e parâmetros $\left\{\alpha_{i}\right\}_{i=1}^{m}$ :

$$
\tilde{\varsigma}(\boldsymbol{x})=\sum_{i=1}^{m} p_{i}(\boldsymbol{x}) \alpha_{i}=\boldsymbol{p}^{T}(\boldsymbol{x}) \boldsymbol{\alpha}_{\omega_{j}}
$$

Observa-se que estão sendo usadas a mesma terminologia e expressões da seção 2.1, pois também aqui o método de ajuste é o dos mínimos quadrados. Este ajuste, contudo, está associado ao nó $\boldsymbol{x}_{j}$ de cada nuvem $\omega_{j}$ em que é calculado. Tal procedimento, em OÑATE (1996a), recebe a denominação de Método dos Mínimos Quadrados Fixo, MMQF. Por esta razão, a função de ponderação é definida com relação ao ponto nodal 
$\boldsymbol{x}_{j}$, e o seguinte funcional do erro passa a ser obtido como:

$$
J_{\omega_{j}}=\sum_{g=1}^{N G} W_{\omega_{j}}\left(\boldsymbol{x}_{g}-\boldsymbol{x}_{j}\right)\left\{\varsigma_{g}-\tilde{\varsigma}\left(\boldsymbol{x}_{g}\right)\right\}^{2}
$$

a partir do qual chega-se, analogamente à seção 2.1:

$$
\boldsymbol{\alpha}_{\omega_{j}}=\sum_{g=1}^{N G} \boldsymbol{A}_{\omega_{j}}^{-1} \boldsymbol{B}_{g}^{\omega_{j}} \varsigma_{g}
$$

onde:

$$
\begin{gathered}
\boldsymbol{A}_{\omega_{j}}=\sum_{g=1}^{N G} W_{\omega_{j}}\left(\boldsymbol{x}_{g}-\boldsymbol{x}_{j}\right) \boldsymbol{p}\left(\boldsymbol{x}_{g}\right) \boldsymbol{p}^{T}\left(\boldsymbol{x}_{g}\right) \\
\boldsymbol{B}_{g}^{\omega_{j}}=W_{\omega_{j}}\left(\boldsymbol{x}_{g}-\boldsymbol{x}_{j}\right) \boldsymbol{p}\left(\boldsymbol{x}_{g}\right)
\end{gathered}
$$

Substituindo-se (I.3) em (I.1) chega-se à seguinte expressão que representa as variáveis $\varsigma$ na nuvem $\omega_{j}$ :

$$
\tilde{\varsigma}_{\omega_{j}}(\boldsymbol{x})=\boldsymbol{p}^{T}(\boldsymbol{x}) \boldsymbol{\alpha}_{\omega_{j}}(\boldsymbol{x})
$$

Considera-se, agora, um elemento quadrilátero $\mathcal{K} \in\left\{\omega_{1} \cap \omega_{2} \cap \omega_{3} \cap \omega_{4}\right\}$, em que as nuvens $\omega_{j}$ estão associadas aos seus quatro vértices $\left\{\boldsymbol{x}_{i}\right\}_{i=1}^{4}$. No interior desse elemento, encontram-se definidas quatro expressões diferentes para (I.6), $\left\{\tilde{\zeta}^{j}\right\}_{j=1}^{4}$. Para se chegar a uma expressão única, podem ser utilizadas as funções de PU, $\left\{\mathcal{N}_{j}\right\}_{j=1}^{4}$, associadas aos quatro vértices, da seguinte maneira:

$$
\tilde{\varsigma}=\sum_{j=1}^{4} \mathcal{N}_{j} \tilde{\varsigma}^{j}
$$

As expressões obtidas isoladamente para cada nuvem são, portanto, "costuradas", de modo equivalente às funções aproximadoras enriquecidas do MEFG. Tem-se então uma expressão contínua para todo o domínio que pode ser utilizada para transferir o conjunto de valores $\left\{\varsigma_{j}\right\}_{j=1}^{N G}$ para o passo seguinte do procedimento adaptativo em análise não-linear. 


\section{Apêndice J}

\section{Solução na Vizinhança de Trinca em Elasticidade Bi-Dimensional}

De acordo com SZABÓ; BABUŠKA (1991), o campo de deslocamentos em $\mathbb{R}^{2}$, na proximidade da ponta da trinca pode ser escrito na forma da seguinte série infinita:

$$
\begin{aligned}
& u_{x}(r, \theta)=\sum_{\alpha=1}^{\infty}\left(A_{\alpha}^{(1) \alpha} u_{x}^{(1)}+A_{\alpha}^{(2) \alpha} u_{x}^{(2)}\right)+\bar{u}_{x}(r, \theta) \\
& u_{y}(r, \theta)=\sum_{\alpha=1}^{\infty}\left(A_{\alpha}^{(1) \alpha} u_{y}^{(1)}+A_{\alpha}^{(2) \alpha} u_{y}^{(2)}\right)+\bar{u}_{y}(r, \theta)
\end{aligned}
$$

onde os índices (1) e (2) referem-se aos modos $I$ e $I I$ de fratura, $r$ e $\theta$ são as coordenadas polares, $r=0$ corresponde à extremidade da trinca, $\bar{u}_{x}(r, \theta)$ e $\bar{u}_{y}(r, \theta)$ são funções mais suaves do que os demais termos denominados auto-funções e dados por:

$$
\begin{aligned}
{ }^{\alpha} u_{x}^{(1)}(r, \theta) & =\frac{r^{\lambda_{\alpha}}}{2 G}\left\{\left[\kappa-Q_{\alpha}^{(1)}\left(\lambda_{\alpha}+1\right)\right] \cos \lambda_{\alpha} \theta-\lambda_{\alpha} \cos \left(\lambda_{\alpha}-2\right) \theta\right\} \\
{ }^{\alpha} u_{x}^{(2)}(r, \theta) & =\frac{r^{\lambda_{\alpha}}}{2 G}\left\{\left[\kappa-Q_{\alpha}^{(2)}\left(\lambda_{\alpha}+1\right)\right] \operatorname{sen} \lambda_{\alpha} \theta-\lambda_{\alpha} \operatorname{sen}\left(\lambda_{\alpha}-2\right) \theta\right\} \\
{ }^{\alpha} u_{y}^{(1)}(r, \theta) & =\frac{r^{\lambda_{\alpha}}}{2 G}\left\{\left[\kappa+Q_{\alpha}^{(1)}\left(\lambda_{\alpha}+1\right)\right] \operatorname{sen} \lambda_{\alpha} \theta+\lambda_{\alpha} \operatorname{sen}\left(\lambda_{\alpha}-2\right) \theta\right\} \\
{ }^{\alpha} u_{y}^{(2)}(r, \theta) & =\frac{r^{\lambda_{\alpha}}}{2 G}\left\{\left[\kappa+Q_{\alpha}^{(2)}\left(\lambda_{\alpha}+1\right)\right] \cos \lambda_{\alpha} \theta-\lambda_{\alpha} \cos \left(\lambda_{\alpha}-2\right) \theta\right\}
\end{aligned}
$$

com os autovalores $\lambda_{1}=1 / 2$ e $\lambda_{\alpha}=(\alpha+1) / 2$ para $\alpha \geq 2$ e:

$$
\begin{gathered}
Q_{\alpha}^{(1)}=\left\{\begin{array}{cc}
-1 & \alpha=3,5,7, \ldots \\
\Lambda_{\alpha} & \alpha=1,2,4,6, \ldots
\end{array} \quad Q_{\alpha}^{(2)}=\left\{\begin{array}{cc}
-1 & \alpha=1,2,4,6, \ldots \\
\Lambda_{\alpha} & \alpha=3,5,7, \ldots
\end{array}\right.\right. \\
\Lambda_{\alpha}=\frac{\lambda_{\alpha}-1}{\lambda_{\alpha}+1} .
\end{gathered}
$$


Sendo $\kappa=\left\{\begin{array}{cc}3-4 \nu & E P D \\ \frac{3-\nu}{1+\nu} & E P T\end{array}, G=\frac{E}{2(1+\nu)}, E\right.$ o módulo de Young e $\nu$ o coeficiente de Poisson. Já os coeficientes $A_{1}^{(1)}$ e $A_{1}^{(2)}$, estão relacionados aos fatores de intensidade de tensão $K_{I}$ e $K_{I I}$ dos modos $I$ e $I I$ de fratura respectivamente:

$$
A_{1}^{(1)}=\frac{K_{I}}{\sqrt{2 \pi}} \quad A_{1}^{(2)}=\frac{K_{I I}}{\sqrt{2 \pi}}
$$

Seja no Método das Nuvens $h p$, ODEN; DUARTE (1997b), como também no MEFG, DUARTE (2001), o seguinte conjunto de funções pode ser empregado para o enriquecimento das funções de PU próximas à extremidade da trinca:

$$
\begin{aligned}
& \mathcal{J}_{j}^{x}=\left\{1,\left\{1-\frac{{ }^{\alpha} u_{x}^{(1)}(\boldsymbol{x})}{{ }^{\alpha} u_{x}^{(1)}\left(\boldsymbol{x}_{j}\right)}\right\}_{\alpha=1}^{\infty},\left\{1-\frac{{ }^{\alpha} u_{x}^{(2)}(\boldsymbol{x})}{{ }^{\alpha} u_{x}^{(2)}\left(\boldsymbol{x}_{j}\right)}\right\}_{\alpha=1}^{\infty}, p_{1}, p_{2}, \cdots, p_{q_{j}(p)}\right\} \\
& \mathcal{J}_{j}^{y}=\left\{1,\left\{1-\frac{{ }^{\alpha} u_{y}^{(1)}(\boldsymbol{x})}{{ }^{\alpha} u_{y}^{(1)}\left(\boldsymbol{x}_{j}\right)}\right\}_{\alpha=1}^{\infty},\left\{1-\frac{{ }^{\alpha} u_{y}^{(2)}(\boldsymbol{x})}{{ }^{\alpha} u_{y}^{(2)}\left(\boldsymbol{x}_{j}\right)}\right\}_{\alpha=1}^{\infty}, p_{1}, p_{2}, \cdots, p_{q_{j}(j)}\right\}
\end{aligned}
$$

As funções de aproximação nas direções $x$ e $y$ para o MEFG são, dessa forma, obtidas conforme as expressões (3.48) e (3.49):

$$
\begin{aligned}
\tilde{u}_{x}(\boldsymbol{x}) & =\sum_{j=1}^{N} N_{j}(\boldsymbol{x})\left\{u_{j}^{x}+\sum_{i=1}^{q_{j}(p)} p_{i} b_{j i}^{x}+\right. \\
& \left.+\sum_{\alpha=1}^{\infty}\left\{\left[1-\frac{{ }^{\alpha} u_{x}^{(1)}(\boldsymbol{x})}{{ }^{\alpha} u_{x}^{(1)}\left(\boldsymbol{x}_{j}\right)}\right] d_{j \alpha}^{x}+\left[1-\frac{{ }^{\alpha} u_{x}^{(2)}(\boldsymbol{x})}{{ }^{\alpha} u_{x}^{(2)}\left(\boldsymbol{x}_{j}\right)}\right] c_{j \alpha}^{x}\right\}\right\} \\
\tilde{u}_{y}(\boldsymbol{x}) & =\sum_{j=1}^{N} N_{j}(\boldsymbol{x})\left\{u_{j}^{y}+\sum_{i=1}^{q_{j}(p)} p_{i} b_{j i}^{y}+\right. \\
& \left.+\sum_{\alpha=1}^{\infty}\left\{\left[1-\frac{{ }^{\alpha} u_{y}^{(1)}(\boldsymbol{x})}{{ }^{\alpha} u_{y}^{(1)}\left(\boldsymbol{x}_{j}\right)}\right] d_{j \alpha}^{y}+\left[1-\frac{{ }^{\alpha} u_{y}^{(2)}(\boldsymbol{x})}{{ }^{\alpha} u_{y}^{(2)}\left(\boldsymbol{x}_{j}\right)}\right] c_{j \alpha}^{y}\right\}\right\}
\end{aligned}
$$

O enriquecimento nodal, assim definido, pode ser usado em todas as nuvens atravessadas pela fratura, desde que essa mantenha-se sempre reta. Para o caso em que a trajetória da trinca apresenta trechos com inclinações variadas deve-se empregar outras estratégias como a introdução de funções do tipo degrau, utilizadas, por exemplo em MOËS; DOLBOW; BELYTSCHKO (1999), ou a modificação da PU fazendo-a descontínua no interior do elemento. Esta última estratégia é sugerida em DUARTE (2001), valendo-se das funções de Shepard juntamente com as funções de interpolação 
Lagrangianas para a construção da PU. A descontinuidade é introduzida aplicando-se o algoritmo wrap-around, DUARTE; ODEN (1996a). Para a definição das funções enriquecedoras em (J.8) e (J.9), outras funções, que não as soluções (J.1) e (J.2), podem também ser empregadas, desde que preservem a singularidade do campo de tensões. Uma interessante proposta pode ser encontrada, por exemplo, em BELYTSCHKO; BLACK (1999). 